\title{
Study of the Rare Decay B Mesons Decaying to X Mesons Positive And Negative Leptons at BABAR
}

\author{
By Ventzislav B. Koptchev
}

Ph.D. Thesis 



\title{
STUDY OF THE RARE DECAY $B \rightarrow X_{s} \ell^{+} \ell^{-}$ AT BABAR
}

\author{
A Dissertation Presented
}

by

VENTZISLAV B. KOPTCHEV

Submitted to the Graduate School of the

University of Massachusetts Amherst in partial fulfillment

of the requirements for the degree of

\section{DOCTOR OF PHILOSOPHY}

June 2004

Department of Physics 
(c) Copyright by Ventzislav B. Koptchev 2004

All Rights Reserved 


\title{
STUDY OF THE RARE DECAY $B \rightarrow X_{s} \ell^{+} \ell^{-}$ AT BABAR
}

\author{
A Dissertation Presented
}

by

\author{
VENTZISLAV B. KOPTCHEV
}

Approved as to style and content by:

Stéphane Y. Willocq, Chair

Carlo Dallapiccola, Member

Eugene Golowich, Member

Grant W. Wilson, Member

Jonathan L. Machta, Department Head

Physics 


\section{ACKNOWLEDGEMENTS}

I have learned a great deal from all I have worked with over the years and gratefully acknowledge my debt to them.

I would like to thank the Experimental High Energy Physics group at the University of Massachusetts - Amherst, for giving me the opportunity to work at a modern high energy physics fascility and for their support throughout my graduate studies. My special thanks go to my adviser, Prof. Stéphane Willocq, for his invaluable help during the initial frustrating period, and expert advice on so many issues related to the analysis and my education in general. I am grateful to Hermann Staengle and Toshinori Abe for their crucial contributions in the areas of signal extraction, background estimates and systematic error evaluation, without their help the timely completion of the analysis would not have been possible.

In the course of my graduate studies I have benefited from numerous lectures I have taken at UMass. I would especially like to thank Prof. Eugene Golowich, whose unique lecturing style has brought back memories of the excitement felt at the time of my first encounters with the wonderful world of Physics.

I would like to thank my primary school physics teacher Asen Tasev for kindling the spark and all the authors, including Paolo Maffei and the late Carl Sagan, who kept it growing.

Finally I would like to thank my parents for the sacrifices and unwavering support that allowed me to pursue my interests. 


\section{ABSTRACT}

Flavor-changing neutral current transitions are forbidden at tree level in the Standard Model and can only occur via higher order diagrams. Since the amplitudes for such loops are dominated by the heaviest known particles, and non-SM effects are expected to contribute at the same order as the SM, such processes are an ideal place to look for new physics.

We present a measurement of the inclusive branching fraction for the flavorchanging neutral current process $B \rightarrow X_{s} \ell^{+} \ell^{-}$with a sample of $81.9 \mathrm{fb}^{-1}$, collected with the BABAR detector at the Stanford Linear Accelerator Center. The final state is reconstructed from $e^{+} e^{-}$or $\mu^{+} \mu^{-}$pairs and a hadronic system consisting of one $K^{ \pm}$or $K_{s}$ and up to two pions, with at most one $\pi^{0}$. We observe a signal of $40 \pm 10$ (stat) \pm 2 (syst) events and extract a branching fraction $\mathcal{B}\left(B \rightarrow X_{s} \ell^{+} \ell^{-}\right)=$ $(5.6 \pm 1.5($ stat $) \pm 0.6($ exp. syst $) \pm 1.1($ model syst $)) \times 10^{-6}$ for $m_{l l}>0.2 \mathrm{GeV}$. 


\section{TABLE OF CONTENTS}

Page

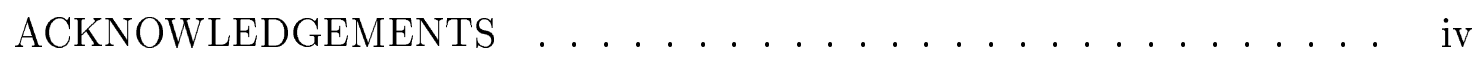

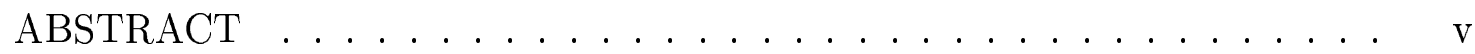

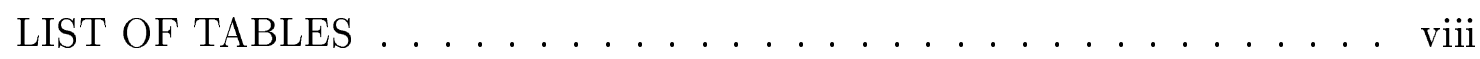

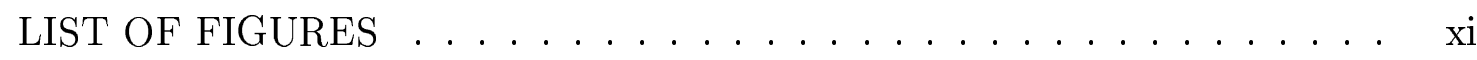
CHAPTER

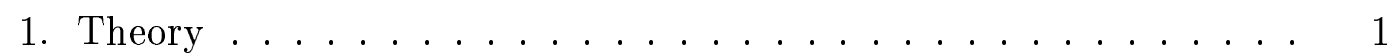

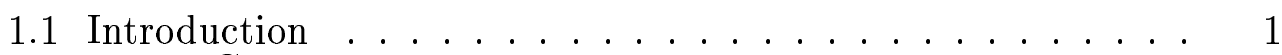

1.2 Model Construction . . . . . . . . . . . . . . . . 4

1.3 Search for New Physics . . . . . . . . . . 13

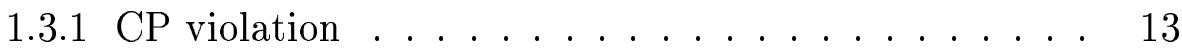

1.3.2 New Physics in Rare B Decays . . . . . . . . . 14

2. The BABAR experiment . . . . . . . . . . . . . 22

2.1 The PEP-II B Factory . . . . . . . . . . . . . . 23

2.2 The BABAR Detector . . . . . . . . . . . . . . . . . 25

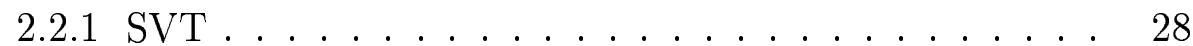

$2.2 .2 \mathrm{DCH} \ldots \ldots \ldots \ldots \ldots \ldots$

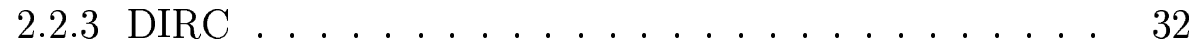

$2.2 .4 \mathrm{EMC} \ldots \ldots \ldots \ldots \ldots \ldots$

2.2 .5 IFR . . . . . . . . . . . . . . 37

2.3 Detector Readout . . . . . . . . . . . . 38

3. Particle Identification . . . . . . . . . . . . . . . 41

3.1 Electron Id . . . . . . . . . . . . . . . . . . . . 41

3.2 Muon Id . . . . . . . . . . . . . . . . . . . . . . . . . . . . 44

3.3 Charged Kaon Id . . . . . . . . . . . . . . . . . . . . . 45

3.4 Neutral Kaon Id . . . . . . . . . . . . . . . . . . . . . 48

3.5 Charged Pion Id . . . . . . . . . . . . . . . . . . 48

3.6 Neutral Pion Id . . . . . . . . . . . . . . . . . . . . . . 49

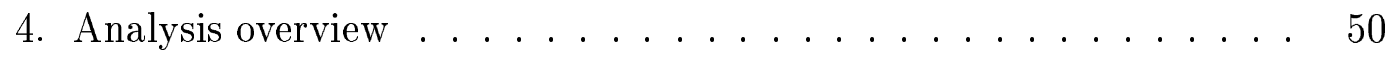

4.1 Monte Carlo generator . . . . . . . . . . . . 50

4.2 Analysis roadmap . . . . . . . . . . . . 54 
5. Signal reconstruction and background suppression $\ldots \ldots \ldots \ldots$

5.1 Event selection . . . . . . . . . . . . . . . . . 56

$5.2 B$ candidate selection $\ldots \ldots \ldots \ldots \ldots \ldots \ldots$

5.3 Background suppression . . . . . . . . . . . 64

5.4 Likelihood ratio cut optimization . . . . . . . . . . . 73

6. Cross checks and validation . . . . . . . . . . . . 85

6.1 Charmonium control sample _. . . . . . . . . . . 85

$6.2 X_{s} e^{ \pm} \mu^{\mp}$ sample . . . . . . . . . . . . . . . . . 102

6.3 Off-resonance sample . . . . . . . . . . . . . . . . 105

6.4 On-resonance sideband sample . . . . . . . . . . . . 105

7. Peaking backgrounds . . . . . . . . . . . . . 115

7.1 Charmonium peaking backgrounds . . . . . . . . . 115

7.2 Hadronic peaking backgrounds . . . . . . . . . . . . 117

8. Maximum likelihood fit . . . . . . . . . . . . . . . 123

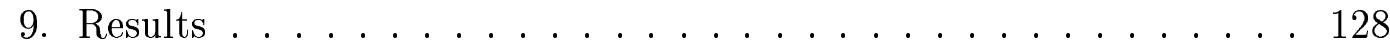

9.1 Total branching fractions $\ldots \ldots \ldots \ldots \ldots$

9.2 Partial branching fractions in dilepton and hadronic mass bins 133

9.3 CP asymmetry . . . . . . . . . . . . . . . . 136

10. Systematic uncertainties _. . . . . . . . . . . . 139

10.1 Signal yield systematics _ . . . . . . . . . . . . . 139

10.2 Branching fraction systematics . . . . . . . . . . . . 140

10.3 CP asymmetry systematics . . . . . . . . . . . . . . 149

11. Summary . . . . . . . . . . . . . . . . . . . 154 APPENDICES

A. Signal efficiency tables . . . . . . . . . . . . . 157

A.1 Background rejection tables . . . . . . . . . . 160

B. Background suppression variables $\ldots \ldots \ldots \ldots \ldots \ldots$

C. Gamma conversion veto . . . . . . . . . . . . . . 181

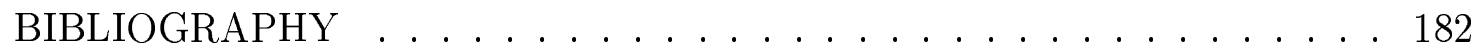




\section{LIST OF TABLES}

Table

Page

1. Electroweak properties of the fundamental matter constituents. . . . 6

2. Branching fraction predictions from Ali et al. [1] . . . . . . . . 19

3. Fractions of $B$ decays in different hadronic final states for the signal model. . . . . . . . . . . . . . 54

4. Monte Carlo samples used in the analysis. . . . . . . . . . 55

5. Functional forms used to fit the nine components of the $\mathcal{L}_{R}$ likelihood. 72

5.1. Signal and background yields for different likelihood ratio cut optimization approaches. The first row shows the results obtained with a single cut, the following rows correspond to separate cuts in different $m_{X s}$ regions, with and without the 3-pion modes. The last row corresponds to the likelihood ratio $\mathcal{L}_{R}^{\prime}$ defined in Eq. 5.4. The likelihood ratio cut values are given on the left-hand side in the order of the $m_{X s}$ ranges. . . . . . . . . . . .

6. Definition of the topology number of the hadronic system $X_{s}$. . . . 86

7. Ratio of background-subtracted $B \rightarrow J / \psi X$ yields for data and MC in the different topologies for the electron channels. . . . . . . . . 95

8. Ratio of background-subtracted $B \rightarrow J / \psi X$ yields for data and MC in the different topologies for the muon channels. . . . . . . . . . 101

9. Ratio of background-subtracted $B \rightarrow \psi(2 S) X$ yields for data and MC in the different topologies for the electron channels. . . . . . . . 101

10. Ratio of background-subtracted $B \rightarrow \psi(2 S) X$ yields for data and MC in the different topologies for the muon channels. . . . . . . . . 102

11. Branching fractions for $B$ decays to $J / \psi$ in SP4 MC (EvtGen) and data (PDG) [2]. The last entry gives the fully inclusive branching fraction at the $\Upsilon(4 S)$ where the MC uncertainty corresponds to the statistical uncertainty obtained in a large sample of generated events. 
12. Branching fractions for $B$ decays to $\psi(2 S)$ in SP4 MC (EvtGen) and data (PDG) [2]. The last entry gives the fully inclusive branching fraction at the $\Upsilon(4 S)$ where the MC uncertainty corresponds to the statistical uncertainty obtained in a large sample of generated

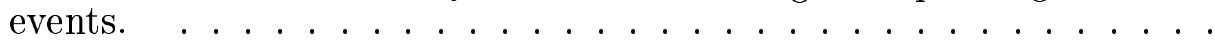

13. Branching fractions for charmonium decays in SP4 MC (EvtGen) and data (PDG) [2]. . . .

14. Number of charmonium peaking background events from Monte Carlo simulation, before applying a cut on the likelihood ratio. The entries are scaled to an integrated luminosity of $81.9 \mathrm{fb}^{-1}$. The numbers given in parentheses are the raw event counts. . . . . . . . . 116

15. Number of charmonium peaking background events from Monte Carlo simulation for electron and muon modes combined in various bins of dilepton and hadronic mass, scaled to an integrated luminosity of $81.9 \mathrm{fb}^{-1}$.

16. Hadronic peaking backgrounds for $X_{s} \mu^{+} \mu^{-}$and its individual event topologies with the $X_{s} h^{+} h^{-}$sample. . . . . . . . . . 119

17. Number of $X_{s} h^{+} h^{-}$hadronic peaking background events for muon modes in various bins of dilepton and hadronic mass. . . . . . . . 122

18. Results of the fit to data: signal yield, peaking background (fixed in the fit), combinatorial background, signal significance, signal efficiency and branching fraction. . . . . . . . . . . . .

19. Signal yields for electron and muon modes combined in various bins of dilepton and hadronic mass. . . . . . . . . . . . . 133

20. Efficiency (in percent) for truth-matched $B \rightarrow X_{s} \ell^{+} \ell^{-}$decays, enhancement factor to account for the cross-feed contribution to the signal yield, and final efficiency (in percent). . . . . . . . .

21. List of systematic uncertainties (in \%) affecting the branching fraction calculation: uncertainties in the signal yield, the signal efficiency, and the number of $B$ mesons.

22. List of systematic uncertainties (in \%) affecting the branching fraction calculation in the different hadronic mass ranges (see Table 19): uncertainties in the signal yield, the signal efficiency, and the number of $B$ mesons. . . . . . . . . . . . . . . . . 147

10.1. List of systematic uncertainties (in \%) affecting the branching fraction calculation in different dilepton mass ranges (see Table 19): uncertainties in the signal yield, the signal efficiency, and the number of $B$ mesons. . . . . . . . . . . . . . . . . . . 148 
23. Apparent $C P$ asymmetry in reconstructed signal events due to tracking, kaon ID and pion ID efficiencies biases. The corresponding statistical error and the error due to tracking, kaon ID and pion ID efficiencies errors are shown for the computed $C P$ asymmetry. . . .

24. Fake $A_{C P}$ in mis-tagged events due to $K^{ \pm} \leftrightarrow \pi^{ \pm}$and $K^{ \pm} \leftrightarrow \mu^{ \pm}$ mis-IDs. The corresponding statistical error and the error due to $K^{ \pm} \leftrightarrow \pi^{ \pm}$and $K^{ \pm} \leftrightarrow \mu^{ \pm}$mis-ID errors are shown for the computed $C P$ asymmetry. . . . . . . . . . . . . .

25. List of fractional systematic uncertainties (in \%) affecting the $A_{C P}$

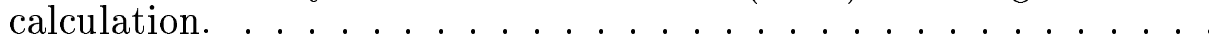

26. Summary of results: signal yield, signal significance, signal efficiency and branching fraction. In the case of the signal yield, the first error is statistical and the second error is systematic. In the case of the signal efficiency, the first error corresponds to the experimental systematic uncertainty arising from detector modeling, hadronization, $B \bar{B}$ counting, and Monte Carlo statistics, whereas the second error corresponds to the uncertainties in the signal model. In the case of the branching fraction, the first, second, and third errors correspond to statistical, experimental systematic, and signal model systematic uncertainties, respectively. ............ 155

27. Summary of branching fractions, where the first error is statistical, the second is systematic excluding signal model uncertainties, and the third is from signal model systematics. . . . . . . . . 155

28. Signal MC efficiency for electron modes. . . . . . . . . . 158

29. Signal MC efficiency for muon modes. . . . . . . . . . . . 159

30. Background $\mathrm{MC}$ rejection for electron modes. The table entries are in the $m_{\mathrm{ES}}$ range $5.20<m_{\mathrm{ES}}<5.29 \mathrm{GeV} \ldots \ldots \ldots 16 \ldots$

31. Background $\mathrm{MC}$ rejection for muon modes. The table entries are in the $m_{\mathrm{ES}}$ range $5.20<m_{\mathrm{ES}}<5.29 \mathrm{GeV} \ldots \ldots \ldots 162$ 


\section{LIST OF FIGURES}

Figure $\quad$ Page

1. The unitarity triangle. . . . . . . . . . . . . . 14

2. Penguin and Box Feynman diagrams for the transition $b \rightarrow s \ell^{+} \ell^{-}$are shown on the left and right, respectively. . . . . . . . . 15

3. Effective "penguin" and "box" vertices in terms of basic vertices. . . 16

4. The dilepton invariant mass distribution in $B \rightarrow K^{*} \mu^{+} \mu^{-}$decays [3]. The solid line represents the SM and the shaded area depicts the form factor-related uncertainties. The dotted line corresponds to the SUGRA model with $R_{7}=-1.2, R_{9}=1.03$ and $R_{10}=1$. The longshort dashed lines correspond to an allowed point in the parameter space of the MIA-SUSY model, given by $R_{7}=-0.83, R_{9}=0.92$ and $R_{10}=1.61$. The corresponding pure SD spectra are shown in the lower part of the plot. . . . . . . . . . . .

5. Differential Forward-Backward asymmetry for the decay $B \rightarrow X_{s} \ell^{+} \ell^{-}$ [1]. The curves correspond to the points indicated in Fig.6. . . . . 21

6. Superposition of all constraints for $\mathrm{C} 9$ and $\mathrm{C} 10[1] \ldots \ldots \ldots$

7. The SLAC Linear Collider, PEP-II and BABAR. . . . . . . . . . 22

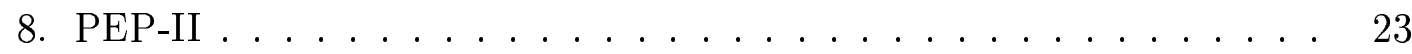

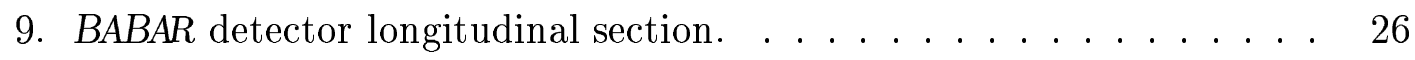

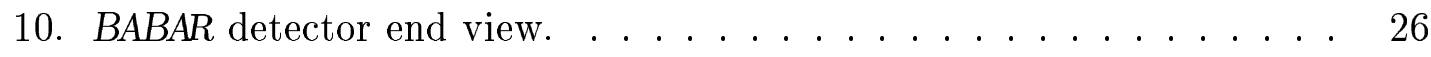

11. The magnetic field components $B_{z}$ and $B_{r}$ as a function of $z$ for various radial distances $r$ (in $\mathrm{m}) . \ldots \ldots \ldots \ldots . \ldots . \ldots 27$

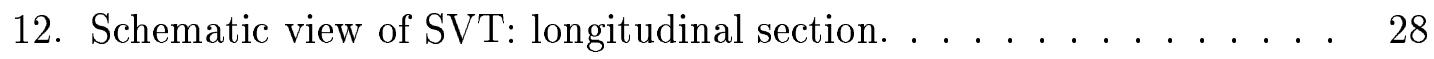

13. Schematic view of SVT: transverse section. . . . . . . . . . 29

14. SVT hit resolution in the a) $z$ and b) $\phi$ coordinate in microns, plotted as a function of track incident angle in degrees. . . . . . . . 30

15. Schematic layout of drift cells for the four innermost superlayers. Lines have been added between field wires to aid in visualization of the cell boundaries. The numbers on the right side give the stereo angles (mrad) of sense wires in each layer. . . . . . . . . . . . . 


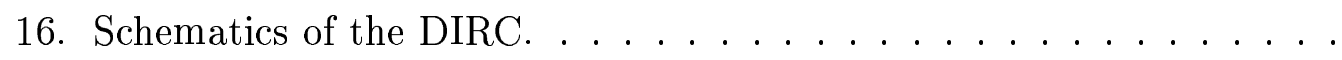

17. Transmission, reflectivity and quantum efficiency for various components of the DIRC as a function of wavelength for a $\beta=1$ particle at normal incidence to the center of a bar . . . . . . . . . .

18. A longitudinal cross section of the EMC (top half). The detector is axially symmetric around the $z$-axis. All dimensions are given in mm. . . . . . . . . . . . . . .

19. Overview of the IFR: Barrel sectors and forward (FW) and backward (BW) end doors; the shape of the RPC modules and their dimensions are indicated. . . . . . . . . . . . . . . .

20. RPC cross section with the schematics of the high voltage connection. 39

21. Schematic diagram of the data acquisition. . . . . . . . . . 40

22. Electron Id efficiency vs momentum. . . . . . . . . . . 43

23. Pion miss-Id vs momentum. . . . . . . . . . . . . . . 43

24. Kaon Id efficiency vs momentum. . . . . . . . . . . . . . . 47

25. Pion miss-Id vs momentum. . . . . . . . . . . . . . . . . 47

26. Hadronic mass distribution of the signal model in the electron channel. The entries are normalized to the expected number in $81.9 \mathrm{fb}^{-1} . . \quad 52$

27. Dilepton mass distribution of the signal model in the electron channel for $m_{l l}>0.2 \mathrm{GeV}$. The entries are normalized to the expected number in $81.9 \mathrm{fb}^{-1}$.

28. Hadronic mass distribution of the signal model in the muon channel. The entries are normalized to the expected number in $81.9 \mathrm{fb}^{-1}$. .

29. Dilepton mass distribution of the signal model in the muon channel. The entries are normalized to the expected number in $81.9 \mathrm{fb}^{-1}$. .

30. Distributions of the number of $B \rightarrow X_{s} e^{+} e^{-}$candidates per event after preselection for $B \rightarrow X_{s} \ell^{+} \ell^{-}$(top left), $B \bar{B}$ (top right), continuum $\mathrm{MC}$ (bottom left), and data events (bottom right). . . . . . . .

31. Distributions of the number of $B \rightarrow X_{s} \mu^{+} \mu^{-}$candidates per event after preselection for $B \rightarrow X_{s} \ell^{+} \ell^{-}$(top left), $B \bar{B}$ (top right), continuum $\mathrm{MC}$ (bottom left), and data events (bottom right). . . . . .

32. Distributions of $\Delta E$ (top), $\log \left(P_{B v t x}\right)$ (middle), and $\cos \theta_{B}$ (bottom) for truth-matched $B \rightarrow X_{s} e^{+} e^{-}$decays (left column), and all other decays (right column), a.k.a. cross-feed, in the signal $B \rightarrow X_{s} \ell^{+} \ell^{-}$

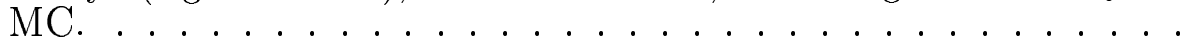


33. Distributions of $\Delta E$ (top), $\log \left(P_{B v t x}\right)$ (middle), and $\cos \theta_{B}$ (bottom) for truth-matched $B \rightarrow X_{s} \mu^{+} \mu^{-}$decays (left column), and all other decays (right column), a.k.a. cross-feed, in the signal $B \rightarrow X_{s} \ell^{+} \ell^{-}$

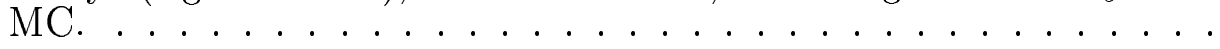

34. Scatter plots of $\Delta E$ vs. dilepton mass in $B \bar{B} \mathrm{MC}$ events for which the leptons of the reconstructed $B \rightarrow X_{s} \ell^{+} \ell^{-}$candidate are either a $J / \psi$ or $\psi(2 S)$ decay products. The muon channel is shown on top and the electron channel is at the bottom. The vertical bands represent the veto regions. The red points indicate fully reconstructed charmonium $B$ decays, while the black ones correspond to charmonium $B$ decays with miss-reconstructed hadronic part. . . . . . . .

35. Distributions of $m_{E S}$ for $B \rightarrow X_{s} e^{+} e^{-}$candidates in $B \bar{B}$ MC events for which the dilepton originates from either a $J / \psi$ or $\psi(2 S)$ decay, before likelihood ratio cut (top) and after (bottom). The left-hand plots correspond to applying a single charmonium veto and those on the right correspond to applying a double veto. . . . . . .

36. Distributions of $m_{\mathrm{ES}}$ for $B \rightarrow X_{s} \ell^{+} \ell^{-}$candidates in $B \bar{B}$ MC events for which the leptons of the reconstructed candidate are either a $J / \psi$ or $\psi(2 S)$ decay products. The muon channel is shown on top and the electron channel is at the bottom. All cuts prior to the charmonium veto are applied on the left and the charmonium veto is included on the right. The red area represents fully reconstructed charmonium $B$ decays and the blue corresponds to charmonium $B$ decays with miss-reconstructed hadronic part. . . . . . . . . . . .

37. Distributions of $m_{E S}$ (top) and dilepton mass (bottom) for $B \rightarrow$ $X_{s} e^{+} e^{-}$candidates in $m_{\mathrm{ES}}<5.27 \mathrm{GeV}$ sideband on-resonance data (points) and MC (histograms). The plots to the right include the likelihood ratio cut. MC distributions have been normalized to correspond to $81.9 \mathrm{fb}^{-1} \ldots \ldots \ldots \ldots \ldots$

38. Distribution of $m_{E S}$ in the electron channel with a particular set of likelihood ratio cuts for the following components in the MC: truthmatched $B \rightarrow X_{s} e^{+} e^{-}$signal (top left), total background including cross-feed (top right), and sum of signal and smoothed background (bottom left). The lines represent the result of a binned maximum likelihood fit to each distribution separately. Entries have been normalized to correspond to $81.9 \mathrm{fb}^{-1} \ldots \ldots$. . . . . . . .

39. Distributions of the likelihood ratio for $B \rightarrow X_{s} e^{+} e^{-}$candidates with $M_{X_{s}}<0.6 \mathrm{GeV}$ in truth-matched signal (top left), cross-feed (top right), $B \bar{B}$ (bottom left) and continuum (bottom right) events. . .

40. Distributions of the likelihood ratio for $B \rightarrow X_{s} e^{+} e^{-}$candidates with $0.6<M_{X_{s}}<1.1 \mathrm{GeV}$ in truth-matched signal (top left), cross-feed (top right), $B \bar{B}$ (bottom left) and continuum (bottom right) events. 
41. Distributions of the likelihood ratio for $B \rightarrow X_{s} e^{+} e^{-}$candidates with $1.1<M_{X_{s}}<1.8 \mathrm{GeV}$ in truth-matched signal (top left), cross-feed (top right), $B \bar{B}$ (bottom left) and continuum (bottom right) events.

42. Distributions of the likelihood ratio for $B \rightarrow X_{s} \mu^{+} \mu^{-}$candidates with $M_{X_{s}}<0.6 \mathrm{GeV}$ in truth-matched signal (top left), cross-feed (top right), $B \bar{B}$ (bottom left) and continuum (bottom right) events. . . 78

43. Distributions of the likelihood ratio for $B \rightarrow X_{s} \mu^{+} \mu^{-}$candidates with $0.6<M_{X_{s}}<1.1 \mathrm{GeV}$ in truth-matched signal (top left), cross-feed (top right), $B \bar{B}$ (bottom left) and continuum (bottom right) events.

44. Distributions of the likelihood ratio for $B \rightarrow X_{s} \mu^{+} \mu^{-}$candidates with $1.1<M_{X_{s}}<1.8 \mathrm{GeV}$ in truth-matched signal (top left), cross-feed (top right), $B \bar{B}$ (bottom left) and continuum (bottom right) events.

45. Distributions of hadronic mass for correctly reconstructed $B \rightarrow X_{s} e^{+} e^{-}$ signal after the nominal likelihood ratio cuts are applied. The selection also excludes final states containing 3 pions. The Belle model is used for the signal MC. . . . . . . . . . . . . .

46. Distributions of hadronic mass for correctly reconstructed $B \rightarrow X_{s} \mu^{+} \mu^{-}$ signal after the likelihood ratio cuts are applied. The selection also excludes final states containing 3 pions. The Belle model is used for the signal MC. ..................

47. Distributions of dilepton invariant mass for $B \rightarrow X_{s} \ell^{+} \ell^{-}$candidates in the charmonium veto sample for electrons (left) and muons (right). The top and bottom rows are for the $J / \psi$ and $\psi(2 S)$ mass regions, respectively. The points represent the data and the histograms the $\mathrm{MC}$, scaled to correspond to an integrated luminosity of $81.9 \mathrm{fb}^{-1}$.

48. Distributions of hadronic invariant mass and final state topology (see Table 6 ) for $B \rightarrow X_{s} \ell^{+} \ell^{-}$candidates in the charmonium veto sample for electrons (left) and muons (right). The points represent the data and the histograms the MC, scaled to correspond to an integrated luminosity of $81.9 \mathrm{fb}^{-1} \ldots \ldots \ldots \ldots$

49. Distributions of $m_{E S}$ for $B \rightarrow X_{s} \ell^{+} \ell^{-}$candidates in the charmonium veto sample for electrons (left) and muons (right). The top row corresponds to data and the bottom shows the MC, scaled to correspond to an integrated luminosity of $81.9 \mathrm{fb}^{-1}$. . . . . . .

6.1. Distributions of the background-suppression variables entering the likelihood ratio for the background-subtracted charmonium events in the electron channel. The points represent the data and the histograms the $\mathrm{MC}$, scaled to correspond to an integrated luminosity

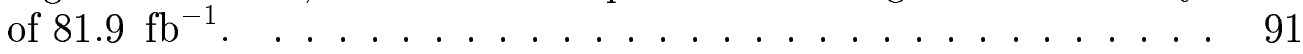


6.2. Distributions of the background-suppression variables entering the likelihood ratio for the background-subtracted charmonium events in the muon channel. The points represent the data and the histograms the MC, scaled to correspond to an integrated luminosity of 81.9 $\mathrm{fb}^{-1} \ldots \ldots \ldots \ldots \ldots \ldots$

50. Distributions of total event energy, lepton momentum (both in the lab frame), and likelihood ratio for $B \rightarrow X_{s} \ell^{+} \ell^{-}$candidates in the charmonium veto sample for electrons (left) and muons (right) after background subtraction. The points represent the data and the histograms the MC, scaled to correspond to an integrated luminosity

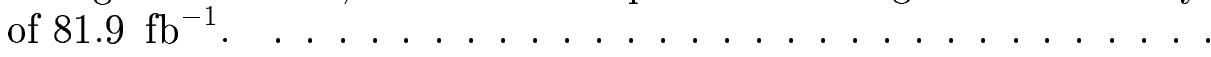

51. Distributions of hadronic invariant mass and final state topology (see Table 6 ) for $B \rightarrow X_{s} \ell^{+} \ell^{-}$candidates in the charmonium veto sample for electrons (left) and muons (right) after background subtraction. The points represent the data and the histograms the MC, scaled to correspond to an integrated luminosity of $81.9 \mathrm{fb}^{-1} \ldots . .$. .

52. Distributions of dilepton invariant mass for $B \rightarrow X_{s} \ell^{+} \ell^{-}$candidates in the charmonium veto sample for electrons (left) and muons (right) after likelihood ratio cuts and background subtraction. The top and bottom rows are for the $J / \psi$ and $\psi(2 S)$ mass regions, respectively. The points represent the data and the histograms the MC, scaled to correspond to an integrated luminosity of $81.9 \mathrm{fb}^{-1}$. . . . . .

53. Distributions of hadronic invariant mass and final state topology (see Table 6) for $B \rightarrow X_{s} \ell^{+} \ell^{-}$candidates in the $B \rightarrow J / \psi X$ veto sample for electrons (left) and muons (right) after likelihood ratio cuts and background subtraction. The points represent the data and the histograms the MC, scaled to correspond to an integrated luminosity of $81.9 \mathrm{fb}^{-1}$. . . . . . . . . . . .

54. Distributions of hadronic invariant mass and final state topology (see Table 6) for $B \rightarrow X_{s} \ell^{+} \ell^{-}$candidates in the $B \rightarrow \psi(2 S) X$ veto sample for electrons (left) and muons (right) after likelihood ratio cuts and background subtraction. The points represent the data and the histograms the MC, scaled to correspond to an integrated luminosity of $81.9 \mathrm{fb}^{-1}$. . . . . . . . . . . .

55. Distributions of total event energy, lepton momentum (both in the lab frame), and likelihood ratio for $B \rightarrow X_{s} \ell^{+} \ell^{-}$candidates in the $B \rightarrow J / \psi X$ veto sample for electrons (left) and muons (right) after likelihood ratio cuts and background subtraction. The points represent the data and the histograms the $\mathrm{MC}$, scaled to correspond to an integrated luminosity of $81.9 \mathrm{fb}^{-1} \ldots \ldots \ldots$. . . . . 
56. Distributions of total event energy, lepton momentum (both in the lab frame), and likelihood ratio for $B \rightarrow X_{s} \ell^{+} \ell^{-}$candidates in the $B \rightarrow \psi(2 S) X$ veto sample for electrons (left) and muons (right) after likelihood ratio cuts and background subtraction. The points represent the data and the histograms the MC, scaled to correspond to an integrated luminosity of $81.9 \mathrm{fb}^{-1} \ldots \ldots \ldots 0$

57. Distributions of $m_{E S}$ for $B$ decay candidates in the $B \rightarrow X_{s} e^{ \pm} \mu^{\mp}$ sample for data (top) and MC (bottom). The MC distribution has been scaled to correspond to an integrated luminosity of $81.9 \mathrm{fb}^{-1}$.

58. Distributions of dilepton mass, hadronic mass, likelihood ratio and final state topology for $B$ decay candidates in the $B \rightarrow X_{s} e^{ \pm} \mu^{\mp}$ sample. The points represent the data and the histograms the $\mathrm{MC}$, scaled to correspond to an integrated luminosity of $81.9 \mathrm{fb}^{-1}$. . . 107

59. Distributions of $m_{E S}$ and dilepton mass for $B \rightarrow X_{s} \ell^{+} \ell^{-}$candidates in the off-resonance data sample for electrons (left) and muons (right). The points represent the data and the histograms the $\mathrm{MC}$, scaled to correspond to an integrated luminosity of $9.6 \mathrm{fb}^{-1} \ldots$. . . . . 108

60. Distributions of hadronic mass and final state topology for $B \rightarrow$ $X_{s} \ell^{+} \ell^{-}$candidates in the off-resonance data sample for electrons (left) and muons (right). The points represent the data and the histograms the MC, scaled to correspond to an integrated luminosity of $9.6 \mathrm{fb}^{-1} \ldots \ldots \ldots \ldots \ldots$. . . . . . . . . . . . . . . . . . . . .

61. Distributions of $m_{E S}$ and dilepton mass for $B \rightarrow X_{s} \ell^{+} \ell^{-}$candidates in the sideband data sample for electrons (left) and muons (right). The points represent the data and the histograms the MC, scaled to correspond to an integrated luminosity of $81.9 \mathrm{fb}^{-1}$. . . . . . 110

62. Distributions of hadronic mass and final state topology for $B \rightarrow$ $X_{s} \ell^{+} \ell^{-}$candidates in the sideband data sample for electrons (left) and muons (right). The points represent the data and the histograms the MC, scaled to correspond to an integrated luminosity of $81.9 \mathrm{fb}^{-1} \ldots \ldots \ldots \ldots \ldots 111 \ldots \ldots \ldots \ldots$

63. Distributions of total event energy, lepton momentum (both in the lab frame) and likelihood ratio for $B \rightarrow X_{s} \ell^{+} \ell^{-}$candidates in the sideband data sample for electrons (left) and muons (right). The points represent the data and the histograms the $\mathrm{MC}$, scaled to correspond to an integrated luminosity of $81.9 \mathrm{fb}^{-1}$. . . . . . 112

6.3. Distributions of the background-suppression variables entering the likelihood ratio for $B \rightarrow X_{s} e^{+} e^{-}$candidates in the sideband data sample. The points represent the data and the histograms the $\mathrm{MC}$, scaled to correspond to an integrated luminosity of $81.9 \mathrm{fb}^{-1}$. . 113 
6.4. Distributions of the background-suppression variables entering the likelihood ratio for $B \rightarrow X_{s} \mu^{+} \mu^{-}$candidates in the sideband data sample. The points represent the data and the histograms the $\mathrm{MC}$, scaled to correspond to an integrated luminosity of $81.9 \mathrm{fb}^{-1}$. . . 114

64. Weighted $m_{E S}$ distribution of $X_{s} h^{+} h^{-}$events. The red line is the total fit to a Gaussian signal plus an Argus background; the blue line is the Argus background portion of the fit. . . . . . . . . . . 119

65. Weighted $m_{E S}$ distributions for $K^{ \pm} n \pi h^{+} h^{-}$final state topologies. The red line is the total fit to a Gaussian signal plus an Argus background; the blue line is the Argus background portion of the fit. . .

66. Weighted $m_{E S}$ distribution for $K_{s}^{0} n \pi h^{+} h^{-}$final state topologies. The red line is the total fit to a Gaussian signal plus an Argus background; the blue line is the Argus background portion of the fit. . .

67. Distributions of $m_{E S}$ for $B \rightarrow X_{s} \ell^{+} \ell^{-}$candidates in the charmonium veto data sample for electrons (top), muons (middle), and both combined (bottom) after all cuts (but the inverted charmonium veto)

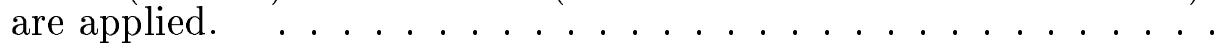

68. Distributions of $m_{E S}$ for truth-matched $B \rightarrow X_{s} \ell^{+} \ell^{-}$candidates for electrons (top) and muons (bottom). . . . . . . . . .

69. Distributions of $m_{E S}$ for data in the (a) $e^{+} e^{-}$, (b) $\mu^{+} \mu^{-}$, (c) $e^{+} e^{-}$ $+\mu^{+} \mu^{-}$, and (d) $e^{ \pm} \mu^{\mp}$ channels. The red line is the result of the fit, with the total background component shown in the dashed (or solid for $\left.e^{ \pm} \mu^{\mp}\right)$ blue line. . . . . . . . . . . . . . . .

70. Distributions of $m_{E S}$ for data in the (a) $e^{+} e^{-}$, (b) $\mu^{+} \mu^{-}$, (c) $e^{+} e^{-}$ $+\mu^{+} \mu^{-}$, and (d) $e^{ \pm} \mu^{\mp}$ channels. The red line is the result of the fit, with the total background component shown in the dashed blue line and the sum of total background + signal cross-feed contribution in the dashed green line; a solid blue line is used for the total background in the $e^{ \pm} \mu^{\mp}$ channel.

71. Distributions of $m_{E S}$ for $B \rightarrow X_{s} \ell^{+} \ell^{-}$candidates in the signal MC that fail truth-matching, i.e. cross-feed events for electrons (top) and muons (bottom). A Gaussian + Argus is used to parametrize the cross-feed PDF for both dilepton modes. . . . . . . . . .

72. Distributions of $m_{E S}$ for $B \rightarrow X_{s} \ell^{+} \ell^{-}$candidates (electron+muon channels combined) in different ranges of dilepton mass (see range in the box above each plot). The red line is the result of the fit, with the total background component shown in the dashed blue line. 134

73. Distributions of $m_{E S}$ for $B \rightarrow X_{s} \ell^{+} \ell^{-}$candidates (electron+muon channels combined) in different ranges of hadronic mass (see range in the box above each plot). The red line is the result of the fit, with the total background component shown in the dashed blue line. 135 
74. Distributions of $m_{E S}$ for combined electron and muon data in selftagged $\bar{B}$ (top) and $B$ (bottom) decays. The red line is the result of the fit, with the total background component shown in the dashed blue line. . . . . . . . . . . . . . . . . . . 138

75. Distributions of $m_{E S}$ for combined electron and muon data in selftagged $\bar{B}$ (top) and $B$ (bottom) decays from the charmonium veto sample. The red line is the result of the fit, with the total background component shown in the dashed blue line. . . . . . . . . 150

76. Number of fitted signal events in bins of $m_{X s}$ (left) and $m_{l l}$ for electron and muon channels combined for data (points) and MC signal (histogram). The error bars represent the statistical uncertainty only. . . . . . . . . . . . . . . . . 156

77. Differential branching fraction as a function of hadronic mass (left) and dilepton mass (right), averaged over electron and muon channels for data (points) and signal Monte Carlo (histogram). . . . . . . . 156

78. Distributions of $\Delta E$ for $B \rightarrow X_{s} e^{+} e^{-}$candidates in truth-matched signal (top left), cross-feed (top right), $B \bar{B}$ (bottom left) and continuum (bottom right) events. The curves show the result of the fits to these distributions using the functions listed in Table $5 . . .$. .

79. Distributions of $\Delta E$ for $B \rightarrow X_{s} \mu^{+} \mu^{-}$candidates in truth-matched signal (top left), cross-feed (top right), $B \bar{B}$ (bottom left) and continuum (bottom right) events. The curves show the result of the fits to these distributions using the functions listed in Table $5 . \ldots . .165$

80. Distributions of $\triangle E^{R O E}$ for $B \rightarrow X_{s} e^{+} e^{-}$candidates in truth-matched signal (top left), cross-feed (top right), $B \bar{B}$ (bottom left) and continuum (bottom right) events. The curves show the result of the fits to these distributions using the functions listed in Table 5. . . . 166

81. Distributions of $\Delta E^{R O E}$ for $B \rightarrow X_{s} \mu^{+} \mu^{-}$candidates in truth-matched signal (top left), cross-feed (top right), $B \bar{B}$ (bottom left) and continuum (bottom right) events. The curves show the result of the fits to these distributions using the functions listed in Table 5. . . . . .

82. Distributions of $m_{E S}^{R O E}$ for $B \rightarrow X_{s} e^{+} e^{-}$candidates in truth-matched signal (top left), cross-feed (top right), $B \bar{B}$ (bottom left) and continuum (bottom right) events. The curves show the result of the fits to these distributions using the functions listed in Table 5. . . . 168

83. Distributions of $m_{E S}^{R O E}$ for $B \rightarrow X_{s} \mu^{+} \mu^{-}$candidates in truth-matched signal (top left), cross-feed (top right), $B \bar{B}$ (bottom left) and continuum (bottom right) events. The curves show the result of the fits to these distributions using the functions listed in Table 5. . . . 169 
84. Distributions of $\Delta z$ between the leptons for $B \rightarrow X_{s} e^{+} e^{-}$candidates in truth-matched signal (top left), cross-feed (top right), $B \bar{B}$ (bottom left) and continuum (bottom right) events. The curves show the result of the fits to these distributions using the functions listed in

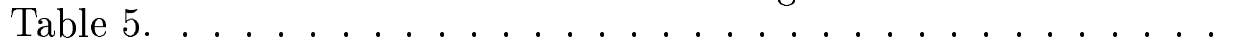

85. Distributions of $\Delta z$ between the leptons for $B \rightarrow X_{s} \mu^{+} \mu^{-}$candidates in truth-matched signal (top left), cross-feed (top right), $B \bar{B}$ (bottom left) and continuum (bottom right) events. The curves show the result of the fits to these distributions using the functions listed in Table 5. . . . . . . . . . . . . . . .

86. Distributions of $\log \left(P_{B v t x}\right)$ for $B \rightarrow X_{s} e^{+} e^{-}$candidates in truthmatched signal (top left), cross-feed (top right), $B \bar{B}$ (bottom left) and continuum (bottom right) events. The curves show the result of the fits to these distributions using the functions listed in Table 5. 172

87. Distributions of $\log \left(P_{B v t x}\right)$ for $B \rightarrow X_{s} \mu^{+} \mu^{-}$candidates in truthmatched signal (top left), cross-feed (top right), $B \bar{B}$ (bottom left) and continuum (bottom right) events. The curves show the result of the fits to these distributions using the functions listed in Table 5. 173

88. Distributions of $\cos \theta_{\text {miss }}$ for $B \rightarrow X_{s} e^{+} e^{-}$candidates in truth-matched signal (top left), cross-feed (top right), $B \bar{B}$ (bottom left) and continuum (bottom right) events. The curves show the result of the fits to these distributions using the functions listed in Table 5. . . . . . 174

89. Distributions of $\cos \theta_{\text {miss }}$ for $B \rightarrow X_{s} \mu^{+} \mu^{-}$candidates in truth-matched signal (top left), cross-feed (top right), $B \bar{B}$ (bottom left) and continuum (bottom right) events. The curves show the result of the fits to these distributions using the functions listed in Table 5. . . . . . 175

90. Distributions of $\cos \theta_{B}$ for $B \rightarrow X_{s} e^{+} e^{-}$candidates in truth-matched signal (top left), cross-feed (top right), $B \bar{B}$ (bottom left) and continuum (bottom right) events. The curves show the result of the fits to these distributions using the functions listed in Table 5. . . . .

91. Distributions of $\cos \theta_{B}$ for $B \rightarrow X_{s} \mu^{+} \mu^{-}$candidates in truth-matched signal (top left), cross-feed (top right), $B \bar{B}$ (bottom left) and continuum (bottom right) events. The curves show the result of the fits to these distributions using the functions listed in Table 5. . . . 177

92. Distributions of $\left|\cos \theta_{T}\right|$ for $B \rightarrow X_{s} e^{+} e^{-}$candidates in truth-matched signal (top left), cross-feed (top right), $B \bar{B}$ (bottom left) and continuum (bottom right) events. The curves show the result of the fits to these distributions using the functions listed in Table 5. . . . . 178 
93. Distributions of $\left|\cos \theta_{T}\right|$ for $B \rightarrow X_{s} \mu^{+} \mu^{-}$candidates in truth-matched signal (top left), cross-feed (top right), $B \bar{B}$ (bottom left) and continuum (bottom right) events. The curves show the result of the fits to these distributions using the functions listed in Table 5. . . . . 179

94. Distributions of $R_{2}$ for $B \rightarrow X_{s} \ell^{+} \ell^{-}$candidates in truth-matched signal (top left), cross-feed (top right), $B \bar{B}$ (bottom left) and continuum (bottom right) events. The curves show the result of the fits to these distributions using the functions listed in Table 5. . . . . 


\section{H A P T E R 1}

\section{Theory}

\section{$1.1 \quad$ Introduction}

The Standard Model (SM) combines the Electroweak theory of Weinberg [4] and Salam [5] with the theory of strong interactions - Quantum Chromodynamics (QCD) - to produce the contemporary theory of elementary particles. The model is based on relativistic quantum field theory, where the Lagrangian formalism is the natural choice, since Lorentz invariance is built in by construction. The Lagrangian function $\mathcal{L}$ is a real, relativistically invariant function depending on the fields and their first derivatives

$$
\mathcal{L}\left(\psi_{i}(x), \partial_{\mu} \psi_{i}(x)\right)
$$

In general, $\mathcal{L}$ contains kinetic terms for each field. In addition, it may contain interaction terms - products of the interacting fields multiplied by a coupling constant, which specifies the strength of the interaction. Mass terms are also possible and are quadratic with respect to the massive field. A field's kinetic term depends on $\partial_{\mu} \psi_{i}(x)$, the exact form being constrained by the requirement of Lorentz invariance and the field's transformation properties under the Lorentz group. Thus, a scalar field representing a spin-0 particle is a singlet under the Lorentz group and 
the kinetic term $\mathrm{T}$ for such a field is

$$
T=\left(\partial^{\mu} \phi(x)\right)\left(\partial_{\mu} \phi(x)\right)
$$

A spinor representing a spin $1 / 2$ particle, transforms as

$$
\psi^{\prime}\left(x^{\prime}\right)=\exp \left(i \epsilon_{\mu \nu} \sigma^{\mu \nu}\right) \psi(x)
$$

where

$$
\sigma^{\mu \nu}=\frac{i}{2}\left[\gamma^{\mu}, \gamma^{\nu}\right]
$$

resulting in a kinetic term

$$
T=\bar{\psi}(x) \gamma^{\mu} \partial_{\mu} \psi(x), \quad \text { with } \bar{\psi}(x) \equiv \psi^{\dagger}(x) \gamma_{0}
$$

A spin-1 particle field $A_{\mu}$, transforms as a Lorentz vector and the kinetic term is introduced via the field strength tensor $F$

$$
T=-\frac{1}{4} F_{\mu \nu} F^{\mu \nu}, F_{\mu \nu} \equiv \partial_{\mu} A_{\nu}-\partial_{\nu} A_{\mu}
$$

In the Standard Model, force mediators appear in the form of spin-1 fields, referred to as gauge fields, which arise when local gauge invariance is imposed on the Lagrangian. Under local transformations represented by a group $G$ of dimension $N$, the matter fields transform as

$$
\psi(x) \rightarrow U \psi(x), \quad \text { with } U(x) \equiv e^{-i \alpha_{a}(x) T^{a}}
$$

where $T_{a}$ form an $N \times N$ representation of the group generators and $\alpha_{a}(x)$ are arbitrary real functions. The requirement of local gauge invariance dictates that all field derivatives in $\mathcal{L}$ must enter as

$$
D_{\mu} \psi(x) \equiv \partial_{\mu} \psi(x)+i g T^{a} W_{\mu}^{a}(x) \psi(x)
$$


where $g$ is a coupling constant and $W^{a}$ are a set of $N$ Lorentz vectors - the gauge fields. The gauge invariant Lagrangian will thus be of the form

$$
\mathcal{L}\left(\psi_{i},\left(\partial_{\mu}+i g T^{a} W_{\mu}^{a}\right) \psi_{i}\right)
$$

and will contain interaction terms between the gauge and the matter fields. The derivative defined in Eq.(1.8) must be covariant under the group transformations

$$
D_{\mu} \psi \rightarrow U D_{\mu} \psi
$$

which imposes the following infinitesimal transformation rule for the gauge fields:

$$
\delta W_{\mu}^{k}=c_{l m k} W_{\mu}^{m} \alpha_{l}(x)+\frac{1}{g} \partial_{\mu} \alpha_{k}(x)
$$

where $c_{l m k}$ are the group's structure constants

$$
\left[T_{j}, T_{k}\right]=i c_{j k l} T_{l}
$$

The gauge invariant kinetic term associated with the gauge fields $W^{a}$ is then

$$
\mathcal{L}_{0}=-\frac{1}{4} F_{\mu \nu}^{j} F^{j, \mu \nu}
$$

where $F$ is the field strength tensor

$$
F_{\mu \nu}^{j} \equiv \partial_{\mu} W_{\nu}^{j}-\partial_{\nu} W_{\mu}^{j}+g c_{j k l} W_{\mu}^{k} W_{\nu}^{l}
$$

which transforms under the adjoint representation of $G$

$$
\delta F_{\mu \nu}^{j}=c_{j k l} \alpha_{k} F_{\mu \nu}^{l}
$$

It follows from Eqs.(1.13 and 1.14), that in a non-Abelian case $\left(c_{j k l} \neq 0\right)$, the gauge fields will be self-coupled. A mass term in the form $W_{\mu} W^{\mu}$, however, cannot be introduced since, according to Eq.(1.11), it would not be gauge invariant. 
In the Standard Model, particle masses are generated through the Higgs Mechanism (spontaneous symmetry breaking in a gauge theory). To employ this mechanism, a number of scalar fields with degenerate ground state are introduced in the Lagrangian, all ground states being related by gauge transformations. The coupling between the gauge bosons and the scalar fields is ensured by the local gauge invariance requirement, through the covariant derivative. The choice of a particular ground state is arbitrary due to the gauge symmetry, however the symmetry is then broken as that state is not invariant under $G$. The state may be invariant under a sub-group of $G$ - $G^{\prime}$ of dimension $N^{\prime}$, the difference $N$ - $N^{\prime}$ indicating the number of broken generators. The physical fields corresponding to interaction mediators are obtained through an appropriate unitary transformation of the initial gauge fields. The ones multiplied by a $G^{\prime}$ generator in the Lagrangian remain massless, the other $N$ - $N^{\prime}$ acquire mass. Expansion of the scalar fields around the chosen ground state, gives rise to $N$-N' massless scalar fields - Goldstone bosons, which are eliminated through an appropriate gauge choice, effectively transferring their degree of freedom to a longitudinal component of the now massive vector bosons. The remaining scalar fields appear as massive Higgs bosons. In Nature, there are 9 unbroken symmetry generators - those associated with electric charge and the strong interaction colors. Hence there are 9 massless mediators, the photon and the 8 strong interaction carriers, the gluons.

\subsection{Model Construction}

When building a Lagrangian for a physical interaction the choice of a gauge symmetry group is motivated by the number of force mediators [6]. For the electromagnetic interaction there is one carrier - the photon and correspondingly a 
group with one generator $U(1)$. The weak interaction is conveyed by three vector bosons, $W^{ \pm}$and $Z^{0}$, and the simplest group with three generators is $S U(2)$. The eight gluon carriers of the strong interaction are represented by $S U(3)$. The matter fields in the Lagrangian represent the fundamental constituents - quarks and leptons to which at least 4 scalar fields have to be added to invoke the Higgs mechanism, since three of the carriers - the weak interaction bosons - are massive. The transformation properties of the matter fields under the chosen gauge group determine their coupling to the gauge bosons. The simplest way to obtain the observed weak-interaction coupling structure is to arrange the leptons and quarks in left-handed $S U(2)$ doublets and right-handed $S U(2)$ singlets, and the 4 scalar fields in a complex $S U(2)$ doublet. Quark and lepton masses are generated by introducing Yukawa terms to the Lagrangian, through which quarks and leptons are coupled to the scalars in a gauge invariant manner. It is regarded that the $U(1)$ and $S U(2)$ transformations are generated by weak hypercharge $Y_{W}$ and weak isospin $I_{W}$, related to the electric charge $Q$, the only unbroken $U(1) \times S U(2)$ generator, by the Gell-Mann - Nishijima relation Eq.(1.16)

$$
Y_{W} / 2=Q-I_{3 W} .
$$

The electroweak interaction properties of the matter fields are summarized in Table 1.

As far as the strong interaction is concerned the quarks are arranged in an $S U(3)$ triplet, the transformations being generated by the quark color. As colorless, all other particles are represented by $S U(3)$ singlets.

The overall gauge group is thus $U(1)_{\text {weak hypercharge }} \times S U(2)_{\text {weak isospin }} \times S U(3)_{\text {color }}$, which we refer to as $U(1)_{Y} \times S U(2)_{I} \times S U(3)_{C}$ in the following. It undergoes spontaneous breaking to $U(1)_{\text {el. charge }} \times S U(3)_{C}$, resulting in one massless gauge boson, 
Table 1: Electroweak properties of the fundamental matter constituents.

\begin{tabular}{cccc}
\hline \hline Particle & $Y_{W}$ & $I_{3 W}$ & $Q$ \\
\hline \hline$\left(\begin{array}{c}\nu_{e} \\
e^{-}\end{array}\right)_{L}\left(\begin{array}{c}\nu_{\mu} \\
\mu^{-}\end{array}\right)_{L}\left(\begin{array}{c}\nu_{\tau} \\
\tau^{-}\end{array}\right)_{L}$ & -1 & $\left(\begin{array}{c}1 / 2 \\
-1 / 2\end{array}\right)$ & $\left(\begin{array}{c}0 \\
-1\end{array}\right)$ \\
$e_{R} \mu_{R} \tau_{R}$ & -2 & 0 & -1 \\
$\left(\begin{array}{l}u \\
d\end{array}\right)_{L}\left(\begin{array}{c}c \\
s\end{array}\right)_{L}\left(\begin{array}{c}t \\
b\end{array}\right)_{L}$ & $1 / 3$ & $\left(\begin{array}{c}1 / 2 \\
-1 / 2\end{array}\right)$ & $\left(\begin{array}{c}2 / 3 \\
-1 / 3\end{array}\right)$ \\
$u_{R} c_{R} t_{R}$ & $4 / 3$ & 0 & $2 / 3$ \\
$d_{R} s_{R} b_{R}$ & $-2 / 3$ & 0 & $-1 / 3$ \\
$\left(\begin{array}{c}\phi^{+} \\
\phi^{0}\end{array}\right)$ & 1 & $\left(\begin{array}{c}1 / 2 \\
-1 / 2\end{array}\right)$ & $\left(\begin{array}{l}1 \\
0\end{array}\right)$ \\
\hline \hline
\end{tabular}

the photon $A_{\mu}$, three massive ones, the $W_{\mu}^{+}, W_{\mu}^{-}$and $Z_{\mu}^{0}$ bosons, and a massive scalar field, the Higgs field. The initial $S U(3)$ gauge symmetry remains unbroken and its gauge bosons unrelated to the electroweak carriers, keeping Quantum Chromodynamics a separate theory.

The initial gauge-invariant electro-weak Lagrangian is

$$
\mathcal{L}_{E W}=\mathcal{L}_{\text {scalar }}+\mathcal{L}_{\text {gauge }}+\mathcal{L}_{\text {matter }}+\mathcal{L}_{Y L}+\mathcal{L}_{Y Q}
$$

The scalar part is

$$
\mathcal{L}_{\text {scalar }}=\left(D^{\mu} \phi\right)^{\dagger}\left(D_{\mu} \phi\right)-V\left(\phi^{\dagger} \phi\right),
$$

where $D_{\mu}$ is the $U(1)_{Y} \times S U(2)_{I}$ covariant derivative

$$
D_{\mu}=\partial_{\mu}+\frac{i g \prime}{2} Y_{W} B_{\mu}+\frac{i g}{2} \vec{\tau} \cdot \overrightarrow{W_{\mu}} .
$$

The 2-dimensional $S U(2)$ generators are the Pauli matrices $\vec{\tau}, g^{\prime}$ and $g$ are the $U(1)_{Y}$ and $S U(2)_{I}$ coupling constants, respectively, $B_{\mu}$ and $\overrightarrow{W_{\mu}}$ are the $U(1)_{Y}$ and $S U(2)_{I}$ gauge bosons, and $\phi$ is the Higgs complex doublet

$$
\phi=\left(\begin{array}{c}
\phi^{+} \\
\phi^{0}
\end{array}\right) .
$$


The potential in Eq.(1.18) is

$$
V\left(\phi^{\dagger} \phi\right)=\mu^{2}\left(\phi^{\dagger} \phi\right)+\lambda\left(\phi^{\dagger} \phi\right)^{2}
$$

with $\lambda>0$ and $\mu^{2}<0$ so that there is a degenerate vacuum state with expectation value $v \equiv \sqrt{\frac{-\mu^{2}}{\lambda}}$. Choosing a vacuum state of the scalar field

$$
\phi_{0}=\left(\begin{array}{c}
0 \\
v / \sqrt{2}
\end{array}\right)
$$

breaks both $U(1)_{Y}$ and $S U(2)_{I}$ symmetries, but preserves an invariance under the $U(1)_{Q}$ generated by the electric charge operator

$$
e^{i \alpha Q} \phi_{0}=\phi_{0}
$$

since

$$
Q \phi_{0}=\frac{1}{2}\left(\tau_{3}+Y\right) \phi_{0}=0 .
$$

Expanding $\phi$ about the minimum of the potential gives

$$
\phi=\exp \left(\frac{i \zeta \cdot \tau}{2 v}\right)\left(\begin{array}{c}
0 \\
(v+H) / \sqrt{2}
\end{array}\right),
$$

where $\zeta$ are the Goldstone boson fields and $H$ is the Higgs scalar field. The exponential factor can be eliminated with a gauge transformation leaving

$$
\phi=\left(\begin{array}{c}
0 \\
(v+H) / \sqrt{2}
\end{array}\right) .
$$

The kinetic term for the gauge fields is

$$
\mathcal{L}_{\text {gauge }}=-\frac{1}{4} F_{\mu \nu}^{l} F^{l \mu \nu}-\frac{1}{4} f_{\mu \nu} f^{\mu \nu}
$$

and the field strength tensors are

$$
F_{\mu \nu}^{l}=\partial_{\mu} W_{\nu}^{l}-\partial_{\nu} W_{\mu}^{l}+g \epsilon_{j k l} W_{\mu}^{j} W_{\nu}^{k}
$$


for the $S U(2)_{I}$ gauge fields and

$$
f_{\mu \nu}=\partial_{\mu} B_{\nu}-\partial_{\nu} B_{\mu}
$$

for the $U(1)_{Y}$ gauge field. The matter term is

$$
\mathcal{L}_{\text {matter }}=\bar{R} i \gamma^{\mu}\left(\partial_{\mu}+\frac{i g \prime}{2} B_{\mu} Y_{W}\right) R+\bar{L} i \gamma^{\mu} D_{\mu} L,
$$

where $L$ denotes a left-handed $S U(2)_{I}$ doublet

$$
L \equiv Q_{i L}, L_{i L}
$$

and $R$ denotes a right-handed $S U(2)_{I}$ singlet

$$
R \equiv U_{i R}, D_{i R}, L_{i R},
$$

with

$$
\begin{gathered}
Q_{i L} \equiv\left(\begin{array}{l}
u \\
d
\end{array}\right)_{L},\left(\begin{array}{c}
c \\
s
\end{array}\right)_{L},\left(\begin{array}{l}
t \\
b
\end{array}\right)_{L}, \\
L_{i L} \equiv\left(\begin{array}{l}
\nu_{e} \\
e
\end{array}\right)_{L},\left(\begin{array}{c}
\nu_{\mu} \\
\mu
\end{array}\right)_{L},\left(\begin{array}{c}
\nu_{\tau} \\
\tau
\end{array}\right)_{L}, \\
U_{i R} \equiv u_{R}, c_{R}, t_{R}, \\
D_{i R} \equiv d_{R}, s_{R}, b_{R}, \\
L_{i R} \equiv e_{R}, \mu_{R}, \tau_{R},
\end{gathered}
$$

$\nu_{R}$ terms are missing since neutrinos are treated as massless. The interaction part of $\mathcal{L}_{\text {matter }}$ can be summarized in

$$
\mathcal{L}_{I N T}=g^{\prime} J_{B}^{\mu} B_{\mu}+g \vec{J}^{\mu} \cdot \vec{W}_{\mu},
$$

where the currents are

$$
J_{B}^{\mu}=-\frac{1}{2} \bar{R} \gamma^{\mu} Y_{W} R-\frac{1}{2} \bar{L} \gamma^{\mu} Y_{W} L
$$


and

$$
J_{i}^{\mu}=-\frac{1}{2} \bar{L} \gamma^{\mu} \tau_{i} L
$$

The physical fields $W_{+}^{\mu}, W_{-}^{\mu}, Z^{\mu}$ and $A^{\mu}$ corresponding to $W^{+}, W^{-}, Z$ and the photon are linear combinations of the initial gauge fields

$$
\begin{aligned}
A^{\mu} & =\sin \theta_{W} W_{3}^{\mu}+\cos \theta_{W} B^{\mu} \\
Z^{\mu} & =\cos \theta_{W} W_{3}^{\mu}-\sin \theta_{W} B^{\mu} \\
W_{ \pm}^{\mu} & =\frac{1}{\sqrt{2}}\left(W_{1}^{\mu} \mp i W_{2}^{\mu}\right),
\end{aligned}
$$

where $\theta_{W}$ is the weak mixing angle. In terms of them the interactions are

$$
\begin{aligned}
\mathcal{L}_{I N T} & =\frac{g}{2 \sqrt{2}}\left[J_{-}^{\mu} W_{\mu+}+J_{+}^{\mu} W_{\mu-}\right] \\
& +\left[\left(g \cos \theta_{W}+g^{\prime} \sin \theta_{W}\right) J_{3}^{\mu}-g^{\prime} \sin \theta_{W} J_{e m}^{\mu}\right] Z_{\mu} \\
& +\left[g^{\prime} \cos \theta_{W} J_{e m}^{\mu}+\left(g^{\prime} \cos \theta_{W}-g \sin \theta_{W}\right) J_{3}^{\mu}\right] A_{\mu}
\end{aligned}
$$

where the charged current and the electromagnetic current are defined as

$$
\begin{aligned}
J_{+}^{\mu} & =2\left(J_{1}^{\mu}-i J_{2}^{\mu}\right)=2 \bar{U}_{i L} \gamma^{\mu} D_{i L}, \\
J_{-}^{\mu} & =2\left(J_{1}^{\mu}+i J_{2}^{\mu}\right)=2 \bar{D}_{i L} \gamma^{\mu} U_{i L}, \\
J_{e m}^{\mu} & =J_{3}^{\mu}+J_{B}^{\mu}=-\frac{1}{2}\left[\bar{L} \gamma^{\mu}\left(\begin{array}{cc}
1+Y_{W} & 0 \\
0 & -1+Y_{W}
\end{array}\right) L+\bar{R} \gamma^{\mu} Y_{W} R\right] \\
& =-Q \bar{f} \gamma^{\mu} f,
\end{aligned}
$$

where $U_{i L}=\left(u, c, t, \nu_{e}, \nu_{\mu}, \nu_{\tau}\right), D_{i L}=(d, s, b, e, \mu, \tau), Q=I_{3}+Y_{W} / 2$ and $f$ is a fermion field. In order to identify the last term in Eq.(1.42) with the electromagnetic interaction

$$
\mathcal{L}_{I N T}^{e m}=e J_{e m}^{\mu} A_{\mu}
$$

we must impose the relation $e=g^{\prime} \cos \theta_{W}=g \sin \theta_{W}$, where $e$ is the electron charge. 
Thus the interaction Lagrangian takes the form

$$
\begin{aligned}
\mathcal{L}_{I N T} & =\frac{e}{2 \sqrt{2} \sin \theta_{W}}\left(W_{+}^{\mu} J_{\mu-}+W_{-}^{\mu} J_{\mu+}\right) \\
& +\frac{e}{2 \cos \theta_{W} \sin \theta_{W}} Z^{\mu} J_{\mu N C}+e A^{\mu} J_{\mu \text { em }}
\end{aligned}
$$

where the weak neutral current is defined as

$$
\begin{aligned}
J_{N C}^{\mu} & =2\left(J_{3}^{\mu}-\sin ^{2} \theta_{W} J_{e m}^{\mu}\right)=-\bar{L} \gamma^{\mu}\left(\begin{array}{cc}
1 & 0 \\
0 & -1
\end{array}\right) L+2 \sin ^{2} \theta_{W} Q \bar{f} \gamma^{\mu} f \\
& =\bar{f} \gamma^{\mu}\left(2 Q \sin ^{2} \theta_{W}-I_{3}+I_{3} \gamma^{5}\right) f
\end{aligned}
$$

The mass Lagrangian for the gauge bosons results from the covariant derivatives in $\mathcal{L}_{\text {scalar }}$, where after substituting the physical gauge fields and the expression Eq.(1.26) for $\phi$, one gets

$$
\begin{aligned}
M_{W^{ \pm}} & =g v / 2, \\
M_{Z} & =M_{W^{ \pm}} / \cos \theta_{W} .
\end{aligned}
$$

The scalar Lagrangian also contains a mass term for the Higgs field

$$
m_{H}=\sqrt{-2 \mu^{2}}
$$

and interaction terms with the gauge bosons.

The lepton fields acquire mass through the Yukawa term

$$
\mathcal{L}_{Y L}=-G_{i}\left(\bar{L}_{i L} \phi L_{i R}+\bar{L}_{i R} \phi^{\dagger} L_{i L}\right)
$$

where $G_{i}$ are unknown coupling constants. After substituting Eq.(1.26) $\mathcal{L}_{Y L}$ becomes

$$
\mathcal{L}_{Y L}=-G_{i}\left(\bar{\ell}_{i R} \ell_{i L}+\bar{\ell}_{i L} \ell_{i R}\right) \frac{v+H}{\sqrt{2}}=\frac{-G_{i} v}{\sqrt{2}} \bar{\ell}_{i} \ell_{i}-\frac{-G_{i}}{\sqrt{2}} \bar{\ell}_{i} \ell_{i} H
$$


where $\ell_{i}$ is $e, \mu$ or $\tau$ and the leptons have acquired mass $m_{i}=G_{i} v / \sqrt{2}$. The Yukawa term for the quarks is

$$
\mathcal{L}_{Y Q}=-G_{i j}^{u} \bar{Q}_{i L} \bar{\phi} U_{j R}-G_{i j}^{d} \bar{Q}_{i L} \phi D_{j R}+\text { h.c. }
$$

where

$$
\bar{\phi}=i \tau_{2} \phi^{*}=\left(\begin{array}{c}
\bar{\phi}^{0} \\
-\phi^{-}
\end{array}\right)
$$

Local gauge invariance does not require the matrices $G_{i j}^{u / d}$ to be diagonal. In fact, to obtain the observed quark mixing they cannot be. Therefore, after the symmetry breaking, non diagonal mass matrices arise

$$
M_{i j}^{u / d}=\frac{v}{\sqrt{2}} G_{i j}^{u / d}
$$

To determine the quark mass eigenstates, the mass matrices must be diagonalized

$$
\left(\mathrm{U}_{L}^{u / d}\right)^{\dagger} M^{u / d} \mathrm{U}_{R}^{u / d}=M_{d i a g}^{u / d}
$$

where $\mathrm{U}_{L}^{u / d}$ and $\mathrm{U}_{R}^{u / d}$ are unitary matrices linked to the basis change

$$
\left(\begin{array}{c}
u_{L / R} \\
c_{L / R} \\
t_{L / R}
\end{array}\right) \rightarrow \mathrm{U}_{L / R}^{u}\left(\begin{array}{c}
u_{L / R} \\
c_{L / R} \\
t_{L / R}
\end{array}\right),\left(\begin{array}{c}
d_{L / R} \\
s_{L / R} \\
b_{L / R}
\end{array}\right) \rightarrow \mathrm{U}_{L / R}^{d}\left(\begin{array}{c}
d_{L / R} \\
s_{L / R} \\
b_{L / R}
\end{array}\right)
$$

Since $M^{u / d}$ are hermitian by construction, $\mathrm{U}_{L}^{u / d}=\mathrm{U}_{R}^{u / d}$. It is worth noting that $J_{N C}^{\mu}(1.48)$ and $J_{e m}^{\mu}(1.45)$ are flavor diagonal in both the weak and the mass basis. This is the essence of the GIM mechanism and implies absence of flavor-changing neutral current (FCNC) transitions at the tree level. Thus the neutral currents are not affected by the basis change. However, for the hadronic charged currents this leads to

$$
J_{+H A D}^{\mu}=2\left(\bar{u}_{L}, \bar{c}_{L}, \bar{t}_{L}\right) \gamma^{\mu}\left(\mathrm{U}_{L}^{u}\right)^{\dagger} \mathrm{U}_{L}^{d}\left(\begin{array}{c}
d_{L} \\
s_{L} \\
b_{L}
\end{array}\right)
$$




$$
J_{-H A D}^{\mu}=2\left(\bar{d}_{L}, \bar{s}_{L}, \bar{b}_{L}\right)\left(\mathrm{U}_{L}^{d}\right)^{\dagger} \mathrm{U}_{L}^{u} \gamma^{\mu}\left(\begin{array}{c}
u_{L} \\
c_{L} \\
t_{L}
\end{array}\right) .
$$

The unitary matrix

$$
V_{C K M} \equiv\left(\mathrm{U}_{L}^{u}\right)^{\dagger} \mathrm{U}_{L}^{d}
$$

is known as the Cabibbo-Kobayashi-Maskawa (CKM) matrix [7] [8]. Because of the unitarity and the 5 arbitrary phases between the six quarks, there are only four independent parameters in the CKM matrix. They account for 4 of the 18 Standard Model parameters to be determined by experiment, the other 14 being the 9 fermion masses, 3 coupling constants, the Higgs mass and Higgs vacuum expectation value. A convenient representation, in which the matrix elements are expressed as a power series of a small parameter $\lambda$, was introduced by Wolfenstein $(1.61)$.

$V_{C K M}=\left(\begin{array}{ccc}V_{u d} & V_{u s} & V_{u b} \\ V_{c d} & V_{c s} & V_{c b} \\ V_{t d} & V_{t s} & V_{t b}\end{array}\right)=\left(\begin{array}{ccc}1-\frac{\lambda^{2}}{2} & \lambda & A \lambda^{3}(\rho-i \eta) \\ -\lambda & 1-\frac{\lambda^{2}}{2} & A \lambda^{2} \\ A \lambda^{3}(1-\rho-i \eta) & -A \lambda^{2} & 1\end{array}\right)+O\left(\lambda^{4}\right)$.

So far $\left|V_{u d}\right|$ and $\left|V_{u s}\right|$ have been determined precisely in nuclear beta decay and $K \rightarrow \pi \ell \nu$, respectively and $\left|V_{c b}\right|$ has been determined from semileptonic B decays. These give values for the parameters $\lambda=0.22$ and $A=0.84$ accurate to about $1 \%$. The other two parameters however, are still poorly determined. 


\subsection{Search for New Physics}

\subsubsection{CP violation}

In addition to the continuous transformations of the Poincare group, there are three independent discrete transformations which preserve the space-time interval $t^{2}-\vec{x}^{2}$. These are the Charge conjugation, the Parity and the Time reversal. The charge conjugation operator $C$ transforms a particle into its anti-particle, the parity operator $P$ flips the sign of the space components in a 4 -vector and the time reversal operator $T$ changes the 4-vector's time component sign. All three together (i.e. $C P T$ ) represent a fundamental symmetry of Nature. However, they are individually violated in the weak interactions. Parity and Charge conjugation violation were discovered by $\mathrm{Wu}$ et al. [9] and Goldhaber et al. [10], respectively, and are accommodated in the SM with the choice of gauge transformation properties of the left and right-handed matter fields. This results in the charged currents having the form $\bar{f}_{L} \gamma^{\mu} f_{L}=1 / 2 \bar{f} \gamma^{\mu}\left(1-\gamma^{5}\right) f$, which has a vector - axial vector (V-A) structure. CP (and therefore T) violation was first observed in the $K$ system by Christenson et al. [11] and recently confirmed by the BABAR and BELLE collaborations in the $B$ system [12] and [13]. The phenomenon is built in the model through the presence of a non-zero complex phase in the CKM matrix as the weak Hamiltonian then does not commute with the operator T. In 1967 Sakharov suggested that $\mathrm{CPV}$ is necessary to generate the matter-antimatter asymmetry in the Universe [14]. However, despite the huge success of the Standard Model in recent years, this sole source of $\mathrm{CP}$ violation in the theory is not enough to explain the amount of the observed asymmetry.

The phase information in the CKM matrix can be displayed using the so-called 


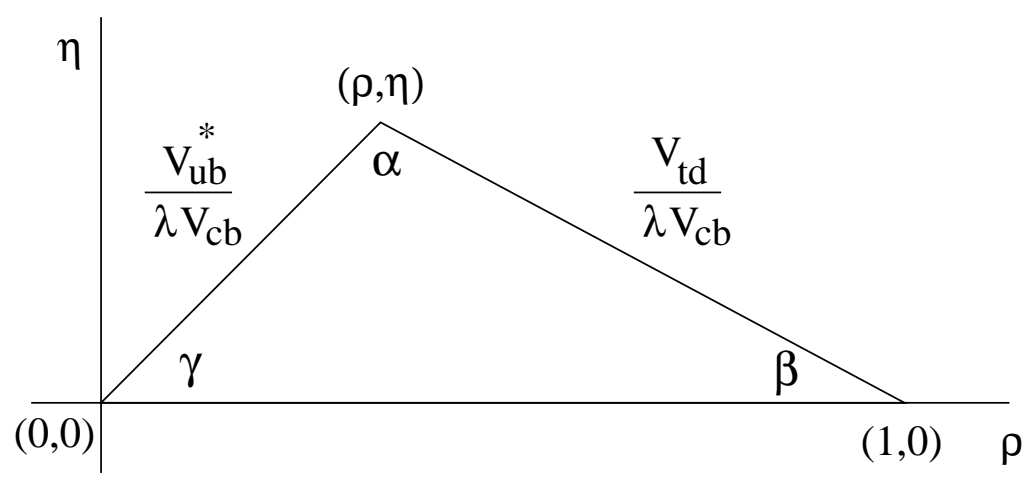

Figure 1: The unitarity triangle.

unitarity triangle (Fig. 1), which follows from the orthogonality of the first and third columns

$$
V_{u d} V_{u b}^{*}+V_{c d} V_{c b}^{*}+V_{t d} V_{t b}^{*}=0,
$$

rescaled by $\left|V_{c d} V_{c b}^{*}\right|=A \lambda^{3}=\lambda\left|V_{c b}\right|$. The non-zero imaginary component $i \eta$ in $V_{u b}$ and $V_{t d}$ see Eq.(1.61) results in a non-zero area of the triangle. The angles $\alpha, \beta$ and $\gamma$ can be measured via $\mathrm{CP}$ violation in $B$ meson decays. The sides of the triangle can also be measured independently. Any deviation from $\alpha+\beta+\gamma=\pi$ or any inconsistency with the independently measured sides would indicate physics beyond the Standard Model. Another place to look for new physics are rare decays which could provide complementary information to the $\mathrm{CP}$ asymmetry measurements and thus help identifying the source of any new physics.

\subsubsection{New Physics in Rare $B$ Decays}

Although FCNC transitions are forbidden at tree level in the Standard Model, due to the different masses of the same-charge quarks, such processes can occur via one-loop diagrams (see Fig.2), albeit at a very low rate [15][16][17][18]. Due to the 

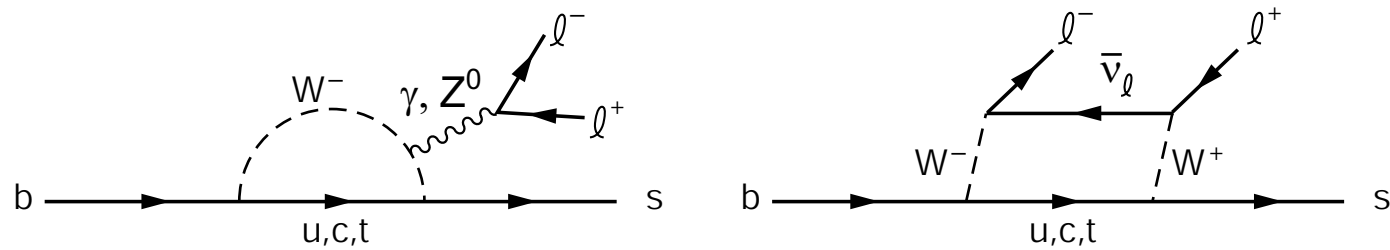

Figure 2: Penguin and Box Feynman diagrams for the transition $b \rightarrow s \ell^{+} \ell^{-}$ are shown on the left and right, respectively.

combinations of CKM matrix elements involved, such loops are quite sensitive to new physics. One-loop processes are possible in $c$ and $t$ decays, but these particles have the CKM-favored decays $c \rightarrow s$ and $t \rightarrow b$ accessible to them. On the other hand since the $s$ and $b$ quarks have no kinematically-allowed CKM-favored decay, the relative importance of the loop decays is greater. Both $s$ and $b$ loop decay amplitudes are dominated by $t$ exchange due to its much higher mass with respect to $u$ and $c$. Since the heaviest known particles $(t, W, Z)$ dominate the amplitudes, rates for these processes are very sensitive to non-SM extensions with for example, heavy charged Higgs or supersymmetric particles. This makes the FCNC processes the most sensitive low energy probes for such extensions to the Standard Model. Searching for new physics in $b$ rather than $s$ decays has some definite advantages, however. Since the $s$ quark mass is relatively small, $s \rightarrow d$ loop diagrams are typically dominated by large non-perturbative effects. In the case of $b \rightarrow s(d)$, due to $\left|V_{t b}\right|$ being close to unity, the loop decays are less suppressed.

At very short distance (high energy) scales $O\left(M_{W, Z, t}\right)$, quark decays are represented by Feynman diagrams (Fig. 2) with $W, Z$ and quark propagators. A picture of a decaying hadron with a mass on the order of $O\left(M_{b, c, s}\right)$ can be described by a set of effective point-like vertices (Fig. 3), represented by local operators $Q_{i}$. The coupling constants associated with those operators are known as the Wilson coef- 

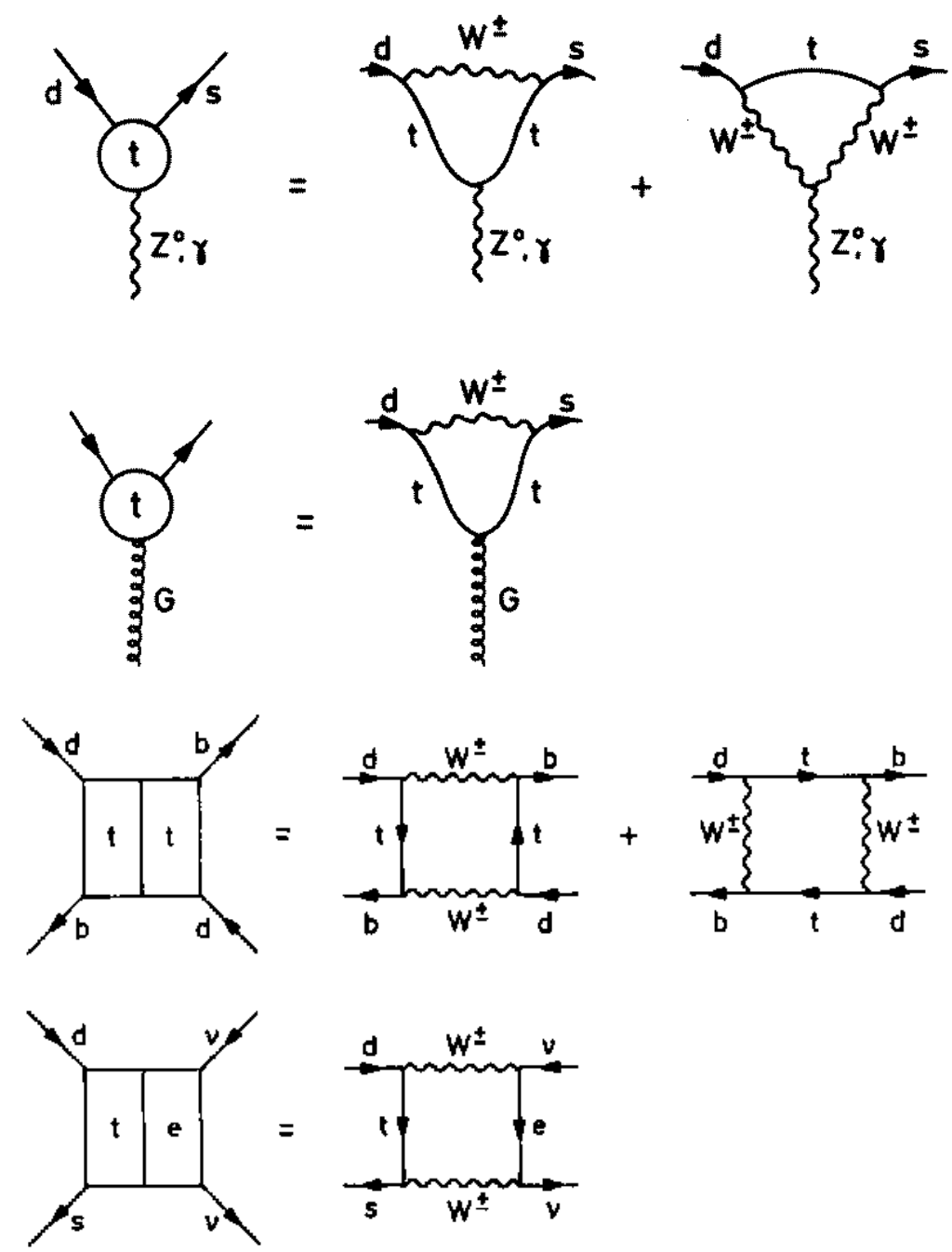

Figure 3: Effective "penguin" and "box" vertices in terms of basic vertices.

ficients $C_{i}$. The effective Hamiltonian describing weak hadron decays can thus be written in the form

$$
H_{e f f}=\frac{G_{F}}{\sqrt{2}} \sum_{i} V_{C K M}^{i} C_{i}(\mu) Q_{i}(\mu),
$$

where $G_{F}$ is the Fermi constant, $\mu$ is a separation energy scale and $Q_{i}$ are the relevant local operators which govern the decays in question. The factors $V_{C K M}$ and the Wilson coefficients $C_{i}$ describe the strength with which a given operator 
enters the Hamiltonian. In the case of beta decay, $H_{\text {eff }}$ is

$$
H_{e f f}^{\beta}=\frac{G_{F}}{\sqrt{2}} \cos \theta_{c}\left[\bar{u} \gamma_{\mu}\left(1-\gamma_{5}\right) d \bar{e} \gamma^{\mu}\left(1-\gamma_{5}\right) \nu_{e}\right],
$$

which corresponds to the Fermi theory of point-like 4-fermion interactions to which the known $u-d$ mixing has been incorporated via the Cabibbo angle $\theta_{c}$. In this particular case the Wilson coefficient is equal to unity and the local operator is given by a product of two currents.

Thus, to obtain an effective low energy theory relevant for scales $O\left(\mu \sim M_{b} \sim\right.$ $5 \mathrm{GeV}$ ), the heavy degrees of freedom $-W, Z^{0}$ and $t$ - must be integrated out, after which they do not appear explicitly in the theory, but their effects are hidden in the Wilson coefficients. The Wilson coefficients $C_{i}(\mu)$ represent the short distance(energy scale higher than $\mu$ ) electroweak interactions and the perturbative (due to asymptotic freedom) strong interactions. To calculate them, they are first obtained at high scale by evaluating the Feynman diagrams with full $W, Z$, $t$ and new particle exchanges and include short-distance $Q C D$ effects. They are then evolved to a low-energy scale using renormalization group equations. This evolution mixes the operators

$$
C_{i}(\mu)=\sum_{j} U_{i j}\left(\mu, M_{W}\right) C_{j}\left(M_{W}\right) .
$$

The renormalization guarantees that the $\mu$ dependence of $C_{i}$ is canceled by the $\mu$ dependence of $Q_{i}$. Since extensions of the Standard Model contribute additional diagrams at the high-energy scale, they modify the values of the Wilson coefficients in the effective low-energy theory. The operator elements $\left\langle X\left|Q_{i}(\mu)\right| B\right\rangle$ summarize the long-distance (energy scale lower than $\mu$ ) non-perturbative strong interactions (here $\mid B>$ represents the $B$ meson and $\mid X>$ the final state it decays into). Unlike the Wilson coefficients, the operator elements cannot be obtained perturbatively due to the confining nature of strong interactions at large 
distances. Non-perturbative methods such as lattice calculations, $Q C D$ sum rules, Heavy Quark Effective Theory(HQET) and so on are used in this case, but they all have limitations. Consequently the dominant theoretical uncertainties in the decay amplitudes reside in the matrix elements $\left\langle Q_{i}(\mu)\right\rangle$. However, when $|X\rangle$ represents an inclusive final state, to leading order $<X_{s}\left|Q_{i}\right| B>\sim<s\left|Q_{i}\right| b>$, where $<s\left|Q_{i}\right| b>$ is an operator element for free quarks which can be calculated perturbatively if no resonant intermediate states are involved. The first nonperturbative corrections are of second order $O\left(1 / M_{b}^{2}\right)$, and are small, thanks to the large $b$ quark mass.

The subject of my study is the inclusive decay $B \rightarrow X_{s} \ell^{+} \ell^{-}$, for which the effective Hamiltonian reads [1]

$$
H_{e f f}=-\frac{4 G_{F}}{\sqrt{2}} V_{t s}^{\star} V_{t b} \sum_{i=1}^{10} C_{i}(\mu) Q_{i}(\mu) .
$$

The first six coefficients $C_{i}(\mu), i=1, \ldots, 6$, determine the non-leptonic $B$-decay rates and the $B$-hadron lifetimes. Since data on $B$ decays and the results obtained in the SM from Eq.(1.66), including QCD effects, are in good agreement with each other $(20 \%)$, there is not much room left for the first six coefficients involving the four-quark operators to deviate from their SM values. The coefficients of interest are $C_{7}(\mu), \ldots, C_{10}(\mu)$, since they are generated at scale $\mu=M_{W}$ by electroweak loops (penguins and boxes). Once extracted from experiment, these can be compared to the theoretical predictions. If there is a deviation from the SM values, new models accounting for the discrepancy can be looked for. If no deviation is observed, the results can be used to set bounds on new physics. In order to measure the values of these coefficients, several observables can be used [19]:

- Invariant dilepton mass distributions $d \mathcal{B}\left(B \rightarrow X_{s} \ell^{+} \ell^{-}\right) / d \hat{s}$, where

$$
\hat{s}=\frac{s}{M_{b}^{2}}, \quad s=\left(p_{\ell^{+}}+p_{\ell^{-}}\right)^{2},
$$


- Forward-backward (FB) charge asymmetry $\mathcal{A}(\hat{s})$ in $B \rightarrow X_{s} \ell^{+} \ell^{-}$, defined with respect to the angular variable $z \equiv \cos \theta$, where $\theta$ is the angle of the $\ell^{+}$with respect to the $b$-quark direction in the center-of-mass system of the dilepton pair.

These quantities can be expressed in terms of the Wilson coefficients

$$
\begin{aligned}
& \frac{d \mathcal{B}\left(B \rightarrow X_{s} \ell^{+} \ell^{-}\right)}{d \hat{s}}= K(1-\hat{s})^{2}\left\{\left(\left|C_{9}^{e f f}(\mu)\right|^{2}+\left|C_{10}(\mu)\right|^{2}\right)(1+2 \hat{s})\right. \\
&\left.+\left|C_{7}(\mu)\right|^{2} \frac{4}{\hat{s}}(2+\hat{s})+12 \operatorname{Re}\left(C_{7}(\mu) C_{9}^{e f f}(\mu)\right)\right\} \\
& \mathcal{A}(\hat{s})=-\frac{3}{2} K(1-\hat{s})^{2} C_{10}(\mu)\left\{C_{9}^{e f f}(\mu) \hat{s}+4 C_{7}(\mu)\right\}
\end{aligned}
$$

where $K$ is a constant and $C_{9}^{e f f}(\mu)=C_{9}(\mu)+f\left(C_{1}(\mu), \ldots, C_{6}(\mu), \hat{s}\right)$. Figures 4 and 5 show dilepton invariant mass and FB asymmetry distributions for several SM extensions as well as the SM predictions.

Recent calculations of the branching fractions are provided in Table 2. In the electron channel, the branching fraction is predicted to be $\mathcal{B}\left(B \rightarrow X_{s} e^{+} e^{-}\right)=$ $(4.2 \pm 0.7) \times 10^{-6}$, for $m\left(e^{+} e^{-}\right)>0.2 \mathrm{GeV}$.

Table 2: Branching fraction predictions from Ali et al. [1]

\begin{tabular}{ll}
\hline \hline Decay & Branching fraction \\
\hline$B \rightarrow K e^{+} e^{-}$ & $(0.35 \pm 0.12) \times 10^{-6}$ \\
$B \rightarrow K^{*} e^{+} e^{-}$ & $(1.58 \pm 0.49) \times 10^{-6}$ \\
$B \rightarrow X_{s} e^{+} e^{-}$ & $(6.89 \pm 1.01) \times 10^{-6}$ \\
$B \rightarrow K \mu^{+} \mu^{-}$ & $(0.35 \pm 0.12) \times 10^{-6}$ \\
$B \rightarrow K^{*} \mu^{+} \mu^{-}$ & $(1.19 \pm 0.39) \times 10^{-6}$ \\
$B \rightarrow X_{s} \mu^{+} \mu^{-}$ & $(4.15 \pm 0.70) \times 10^{-6}$ \\
\hline
\end{tabular}




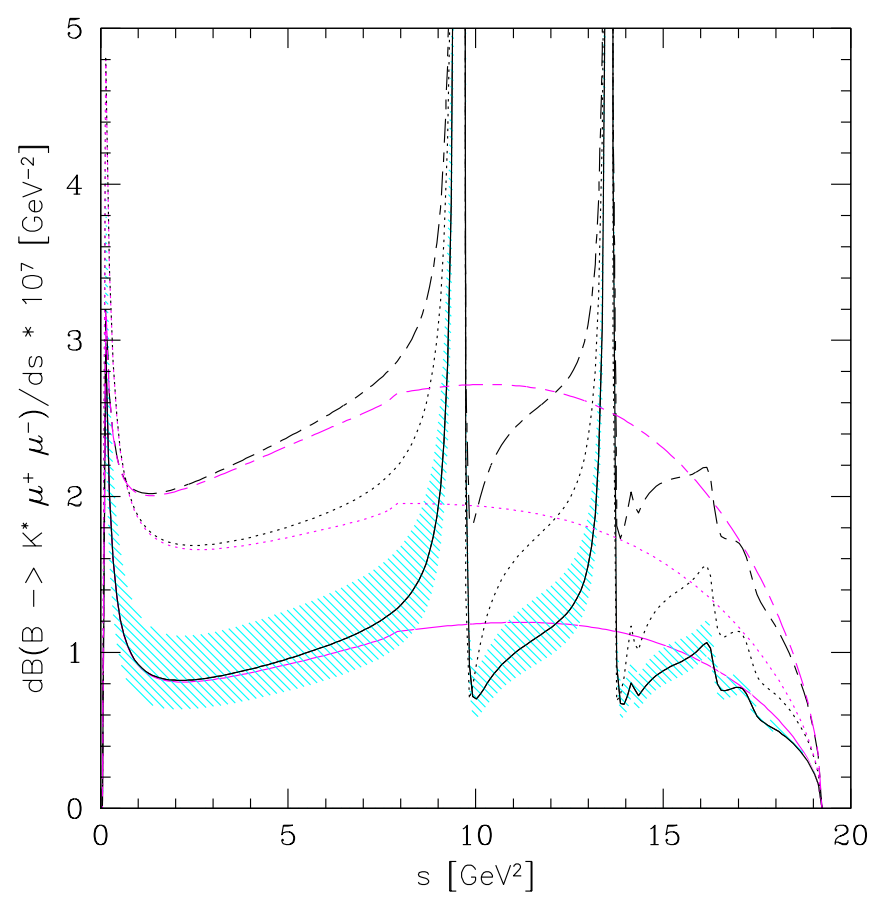

Figure 4: The dilepton invariant mass distribution in $B \rightarrow K^{*} \mu^{+} \mu^{-}$decays [3]. The solid line represents the SM and the shaded area depicts the form factor-related uncertainties. The dotted line corresponds to the SUGRA model with $R_{7}=-1.2, R_{9}=1.03$ and $R_{10}=1$. The longshort dashed lines correspond to an allowed point in the parameter space of the MIA-SUSY model, given by $R_{7}=-0.83, R_{9}=0.92$ and $R_{10}=1.61$. The corresponding pure SD spectra are shown in the lower part of the plot. 


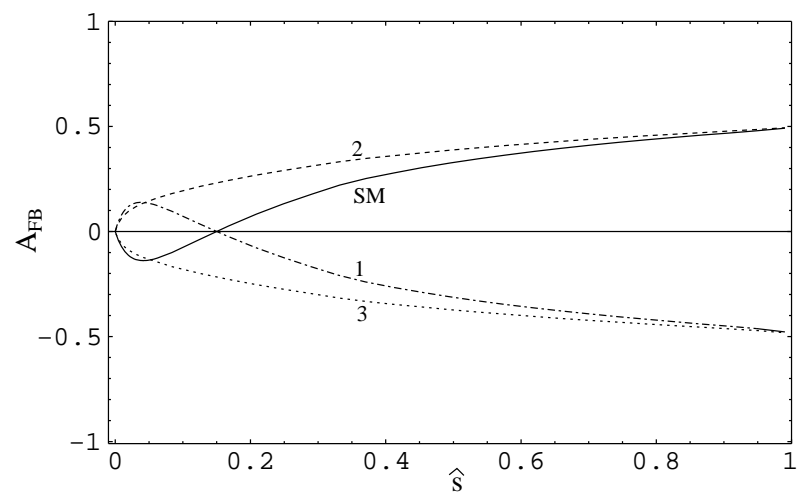

Figure 5: Differential Forward-Backward asymmetry for the decay $B \rightarrow$ $X_{s} \ell^{+} \ell^{-}[1]$. The curves correspond to the points indicated in Fig.6.

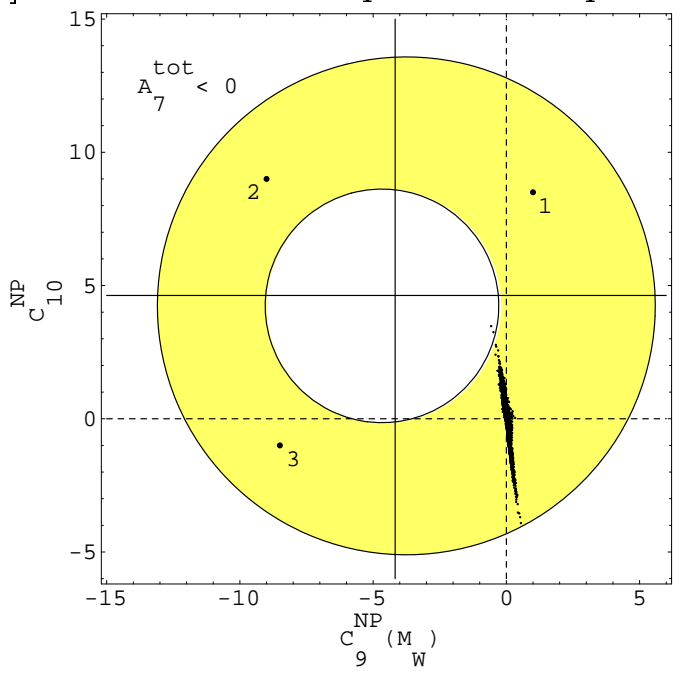

Figure 6: Superposition of all constraints for C9 and C10 [1]. 


\section{H A P T E R 2}

\section{The BABAR experiment}

The goal of the BABAR [20] experiment is to study $B$ meson decays produced by the PEP-II asymmetric electron-positron collider at the Stanford Linear Accelerator Center (SLAC). Although the detector design was prompted by the primary

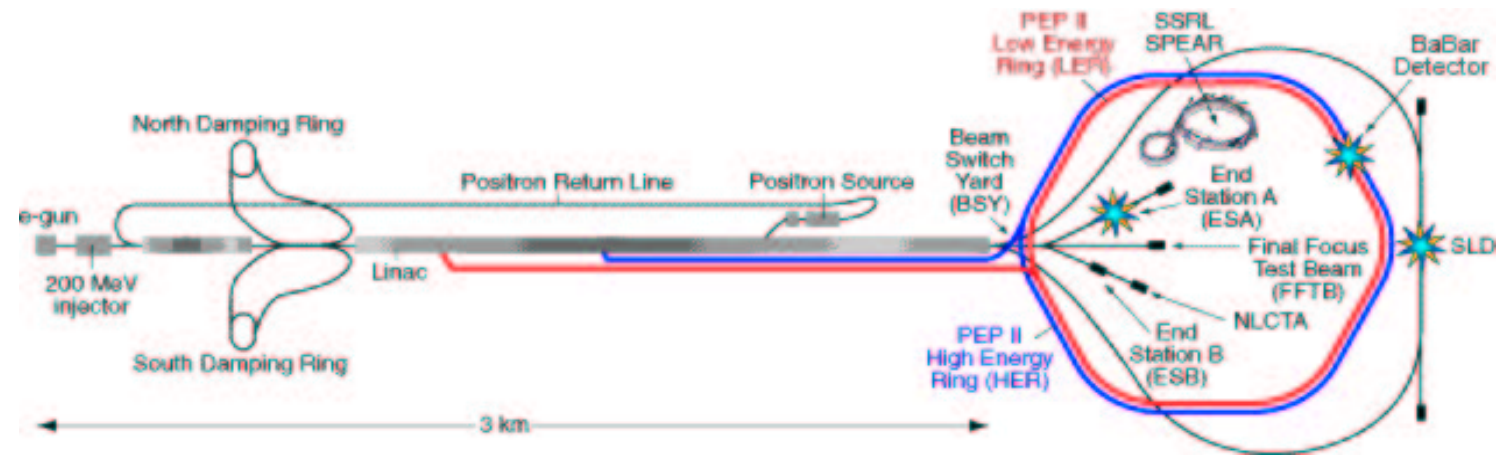

Figure 7: The SLAC Linear Collider, PEP-II and BABAR.

purpose of the experiment - the measurement of $\mathrm{CP}$ violation in the $B$ system - it is versatile enough to make a wide range of $B$ physics studies possible. 


\subsection{The PEP-II B Factory}

The PEP-II $B$ Factory is designed to operate at a luminosity of $3 \times 10^{33} \mathrm{~cm}^{-2} \mathrm{~s}^{-1}$ and above. It consists of two storage rings for the $9.0 \mathrm{GeV}$ electrons and $3.1 \mathrm{GeV}$ positrons located one on top of the other. The electrons go clockwise around the old lower ring, while the positrons go anticlockwise around the newly built upper ring. Being less energetic, the positron beam is diverted into the interaction region. The beam energies are selected to produce the $\Upsilon(4 S)$ resonance with a boost of

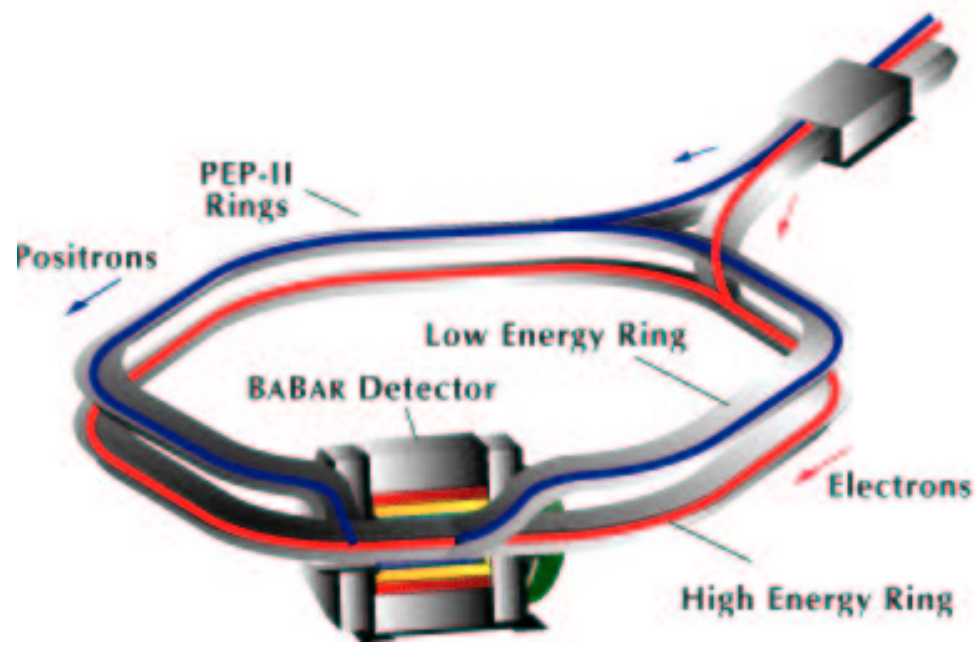

Figure 8: PEP-II

$\beta \gamma=0.56$. This resonance decays exclusively to $B^{0} \bar{B}^{0}$ and $B^{+} B^{-}$pairs and thus provides an ideal laboratory for the study of $B$ mesons. The boost makes it possible to reconstruct the decay vertices of the two $B$ mesons, determine their relative decay times, and thus measure the time dependence of their decay rates. Until recently PEP-II operated on a 40-50 minute cycle with the beams being replenished at the end of each fill. Due to higher backgrounds during injection data taking was halted and some detector components switched off. Since March 2004 a new trickle 
injection method was implemented. With it, new bunches are injected continuously at a rate of up to 10 per second. The average $b \bar{b}$ production rate is thus increased by up to $50 \%$. BABAR divides the data into runs, defined as periods of three hour duration or less during which beam and detector conditions are judged to be stable. While most of the data are recorded at the peak of the $\Upsilon(4 S)$ resonance, about $12 \%$ are taken at a center of mass (c.m.) energy $40 \mathrm{MeV}$ lower to allow for studies of non-resonant (continuum) background. This background is due to light quark (udsc) production the cross section for which, at the resonance, is about three times larger than the one for $\Upsilon(4 S)(1.05 \mathrm{nb})$.

The BABAR coordinate system is right-handed with the z-axis coinciding with the detector principle axis and the positive direction along the electron beam. This axis is offset relative to the beam axis by about $20 \mathrm{mrad}$ in the horizontal plane. The positive $\mathrm{y}$-axis points upward and the positive $\mathrm{x}$-axis points away from the center of the storage rings.

Several beam parameters are crucial for BABAR physics analyses. The mean beam energies are determined from the total magnetic bending strength. They enter in the calculation of $\Delta E=E_{B}^{*}-E_{\text {beam }}^{*}$ and the energy-substituted mass $m_{\mathrm{ES}}=\sqrt{E_{\text {beam }}^{* 2}-p_{B}^{* 2}}$, where $E_{B}^{*}$ and $p_{B}^{*}$ are the energy and 3-momentum of the reconstructed $\mathrm{B}$ candidate and the $*$ indicates that these quantities are measured in the c.m. frame. These two kinematic variables are largely uncorrelated and are widely used to separate signal from background in analyses involving exclusive $B$-meson decays. The use of $E_{\text {beam }}$ rather than $E_{B}$ in the calculation of $m_{\mathrm{ES}}$ results in better resolution. The absolute luminosity is derived from $e^{+} e^{-} \rightarrow e^{+} e^{-}$and $e^{+} e^{-} \rightarrow \mu^{+} \mu^{-}$processes. The position of the luminous region is important for decay-time-dependent analyses. In the transverse plane it is determined from the distribution of the distance of closest approach to the $z$-axis of tracks in well mea- 
sured two-track events as a function of the azimuth $\phi$. The longitudinal position is derived from the longitudinal vertex distribution of the two tracks.

\subsection{The BABAR Detector}

The BABAR detector was built by a large international collaboration of scientists. A longitudinal section through the detector center and an end view are shown on Figures 9 and 10, respectively. Because of the non-zero boost and in order to maximize acceptance, it is mounted asymmetrically with respect to the interaction point (IP). The detector incorporates five sub-systems, each providing complementary information. The charged particle tracking is done with a silicon vertex tracker (SVT) and a drift chamber $(\mathrm{DCH})$. The SVT measures positions and angles of charged particles near the IP, while the main purpose of the DCH is momentum measurement of charged particles and providing additional particle identification information via $d E / d x$. The third detector component is the ringimaging Cherenkov detector (DIRC) and is used for charged particle identification. Photons are detected in the electromagnetic calorimeter (EMC), which is designed to provide excellent energy and angular resolution. The muon and neutral hadron part of the detector (IFR) is an integral part of the magnet flux return. More detailed information about each of these subsystems is provided in the following subsections.

In order to achieve the desired charged particle momentum resolution, the detector is immersed in a magnetic field of $1.5 \mathrm{~T}$. The system consists of a superconducting solenoid, a flux return, a conventional water cooled field compensating coil, to reduce magnetic flux leakage at the backward end to levels acceptable for the PEP-II components and the DIRC photo-multipliers, and a cryostat for the super- 


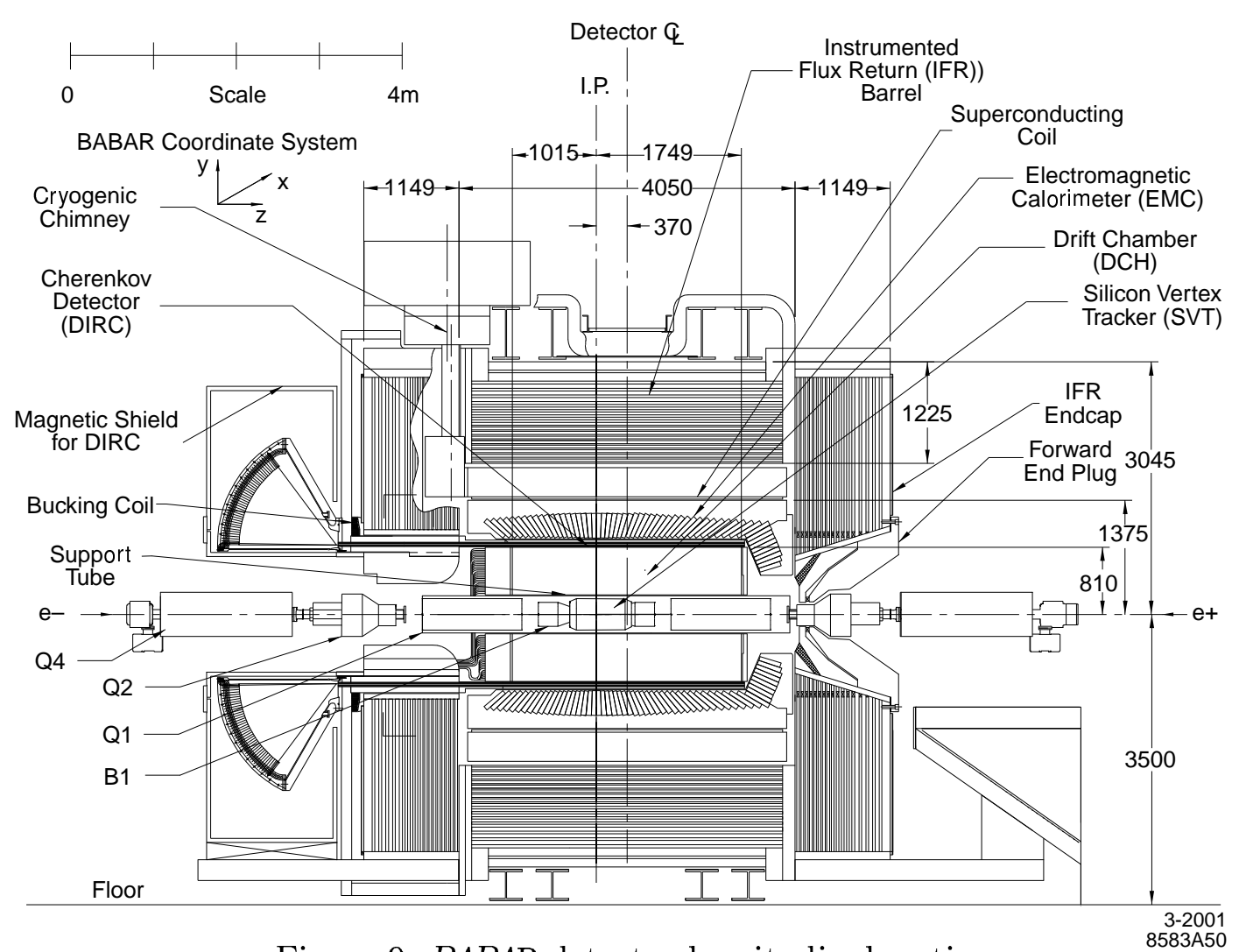

Figure 9: BABAR detector longitudinal section.

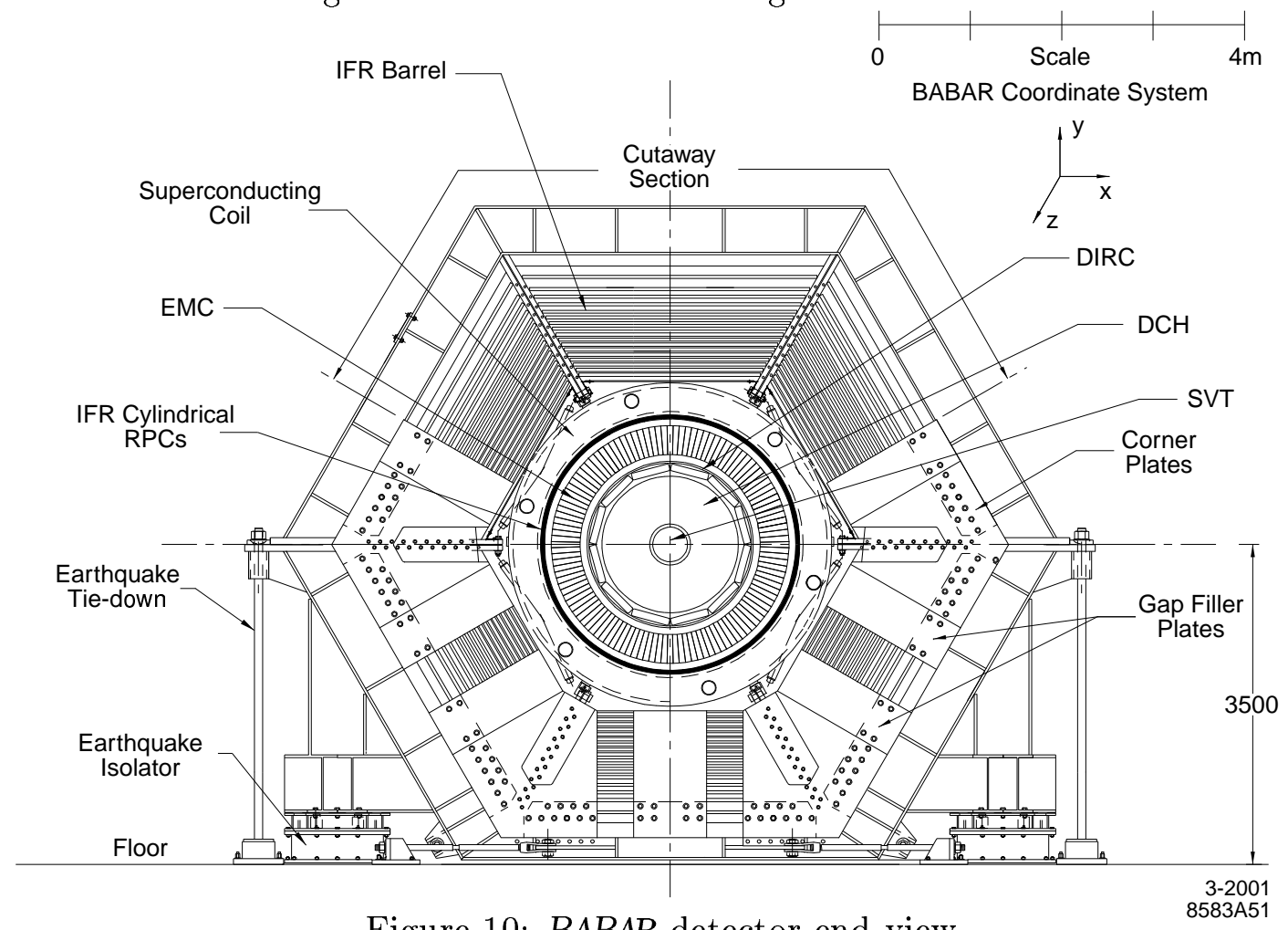

Figure 10: BABAR detector end view. 


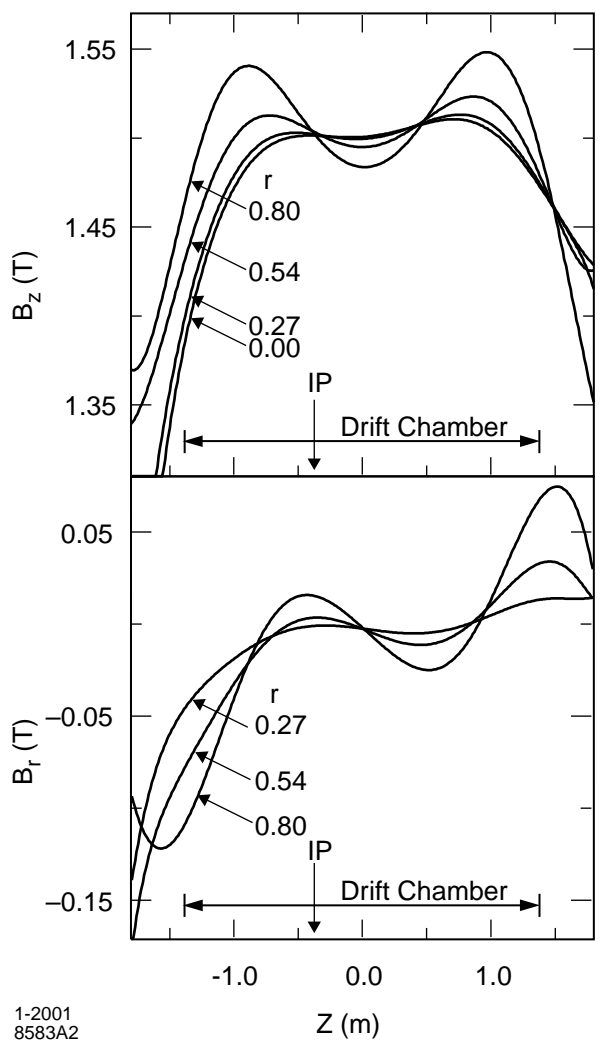

Figure 11: The magnetic field components $B_{z}$ and $B_{r}$ as a function of $z$ for various radial distances $r$ (in $\mathrm{m}$ ).

conducting coil. The direction of the field is along the detector's $\mathrm{z}$ axis, which is also the approximate direction of the electron beam. To facilitate track fitting, the field is uniform to within a few percent in the tracking volume, see Fig. 11. The cryostat uses helium to indirectly cool the superconducting solenoid to an operating temperature of $4.5 \mathrm{~K}$. 


\subsubsection{SVT}

The main goal of the experiment - measurement of time-dependent CPV depends greatly on the SVT and imposes a stringent requirement on the mean vertex resolution of a fully reconstructed B candidate to be better than $80 \mu \mathrm{m}$ along the z-axis. The resolution in the $\mathrm{x}-\mathrm{y}$ plane needs to be approximately 100 $\mu m$ to reconstruct $B^{0} \rightarrow D^{+} D^{-}$decays for example. In addition many $\mathrm{B}$ decay products have low transverse momenta $p_{t}<120 \mathrm{MeV} / \mathrm{c}$, the minimum that can be measured reliably in the $\mathrm{DCH}$ alone. In particular for the identification of slow pions from $D^{*}$ decays, a tracking efficiency of more than $70 \%$ is desirable for tracks with transverse momenta ranging from 50 to $120 \mathrm{MeV} / \mathrm{c}$. Additional requirements on reliability are imposed due to the inaccessibility and the large radiation dosage the SVT must withstand. In order to meet these objectives the SVT incorporates five layers of double-sided silicon strip detectors, Figures 12 and 13 . The first three layers are mounted close to the beam pipe and provide angle and vertex position information. The outer two layers are at larger radii and are used to link the SVT and DCH tracks as well as for standalone tracking for particles with $p_{t}<120 \mathrm{MeV} / \mathrm{c}$. The strips on the opposite sides of each sensor are orthogonal to

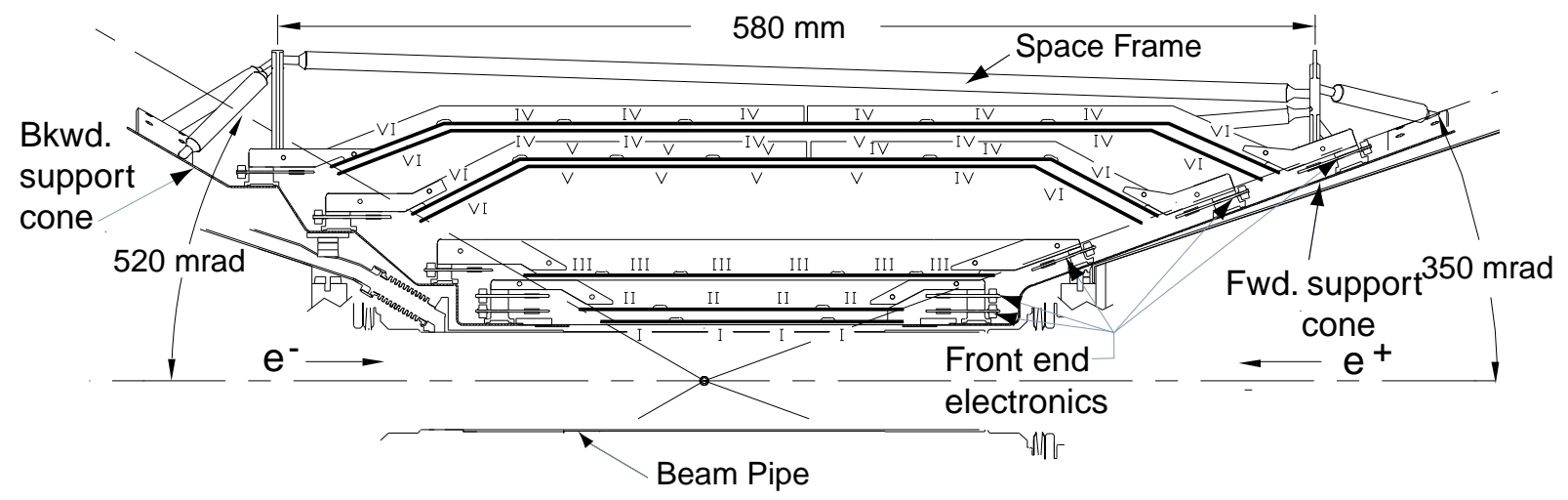

Figure 12: Schematic view of SVT: longitudinal section. 


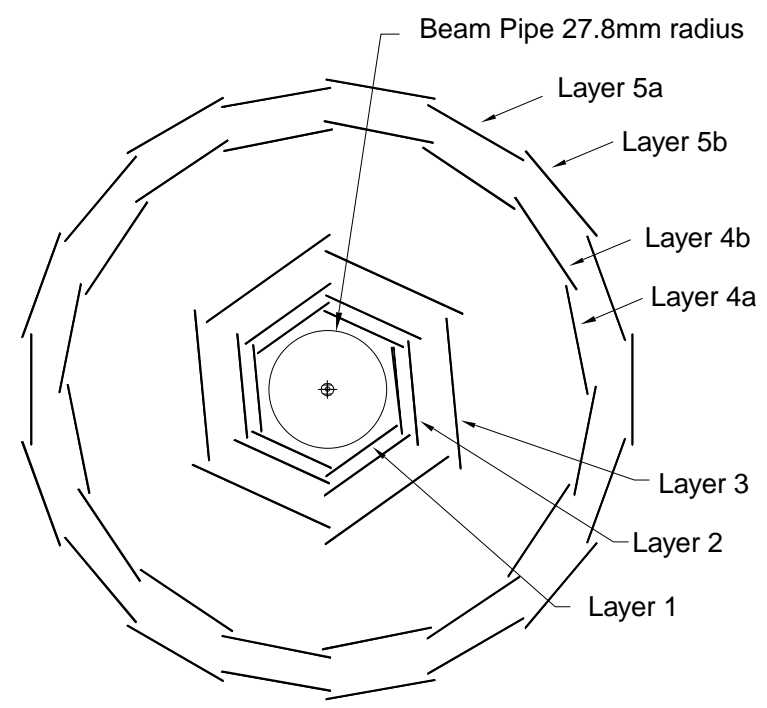

Figure 13: Schematic view of SVT: transverse section.

each other, the $\phi$ measuring strips run parallel to the beam and the $z$ measuring strips are transverse to the beam axis. To maximize acceptance while minimizing detector material the outer two layers have an arch design. The efficiency of each layer can be measured by comparing the number of associated hits to the number of tracks crossing the active area of the layer and is $97 \%$. The hit resolution is determined from high-momentum tracks in two prong events by measuring the distance, in the plane of the sensor, between the track trajectory and the hit, see Fig. 14. 

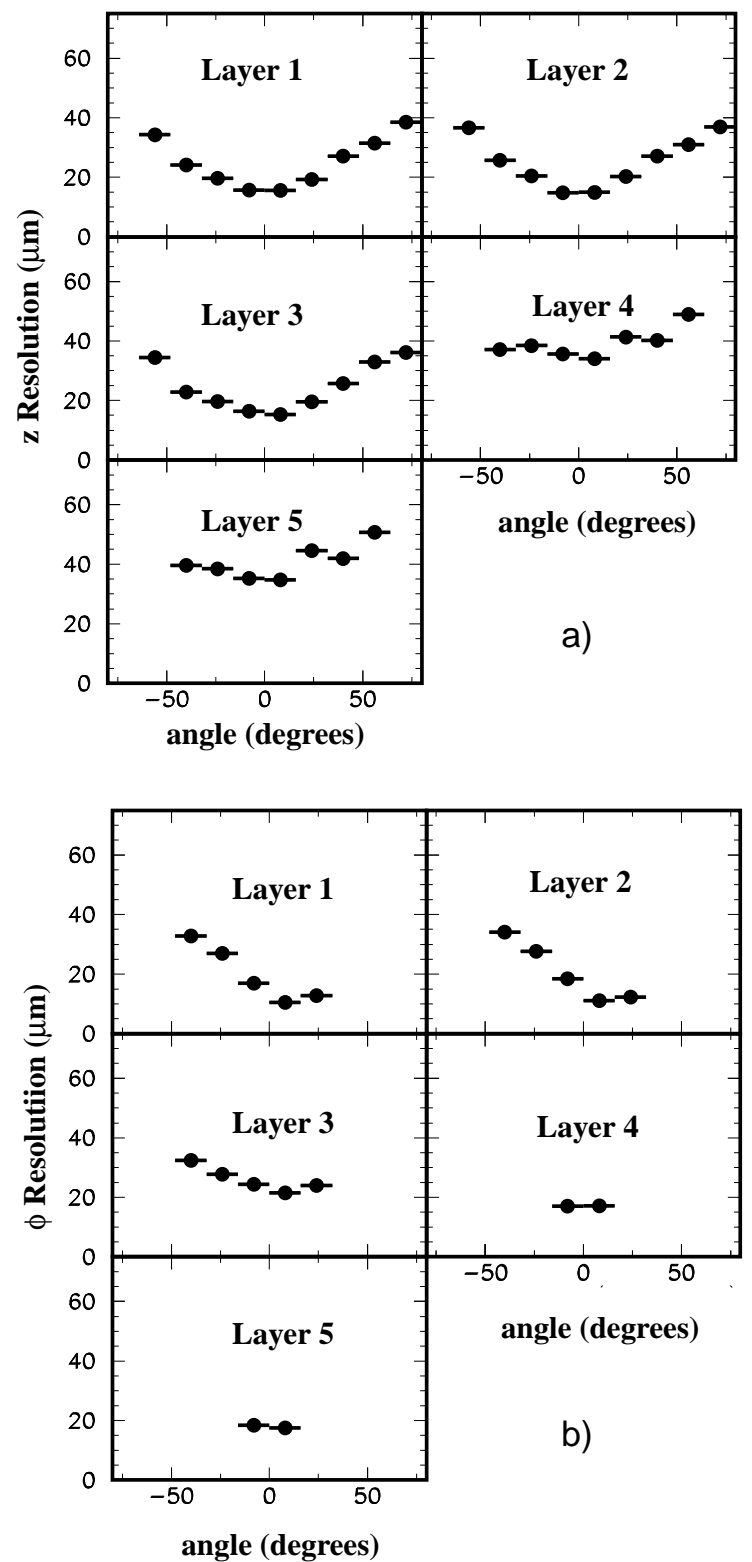

Figure 14: SVT hit resolution in the a) $z$ and b) $\phi$ coordinate in microns, plotted as a function of track incident angle in degrees. 


\subsubsection{DCH}

The DCH detects charged particles and measures their momenta with high precision. In addition it helps $\pi / K$ separation by measuring the ionization loss $d E / d x$. In the barrel this information is complementary to the one from the DIRC, but beyond the DIRC acceptance the $\mathrm{DCH}$ is the only device providing some discrimination. The reconstruction of decay vertices outside of the SVT relies solely on the $\mathrm{DCH}$ as well. For this purpose, in addition to transverse momenta and positions, the chamber should be capable of measuring longitudinal positions with a resolution of $1 \mathrm{~mm}$. The DCH consists of 40 layers of cells, filled with a heliumbased gas mixture, see Fig. 15. The cells are close to hexagonal in shape and consist of one sense wire surrounded by six field wires. A positive voltage of $1960 \mathrm{~V}$ is applied to the sense wires while the field wires are at ground potential. In order to obtain longitudinal information, the wires in 24 of the layers are at small angles with respect to the z-axis. Multiple scattering is minimized by the choice of gas and low-mass aluminum wires. The mixture of helium and isobutane is provided at a stable $80 / 20$ ratio by a gas recirculation system at a constant pressure. During normal operation a complete gas recirculation is done in six hours, and a full volume of fresh gas is added every 36 hours. In order to prevent electrical discharge a relatively high level of humidity is maintained by passing a controlled fraction of the gas through a watter bubbler. Oxygen is removed from the gas mixture with a palladium catalytic filter. The readout electronics are mounted on the back plate of the chamber to minimize material in front of the calorimeter endcap. 


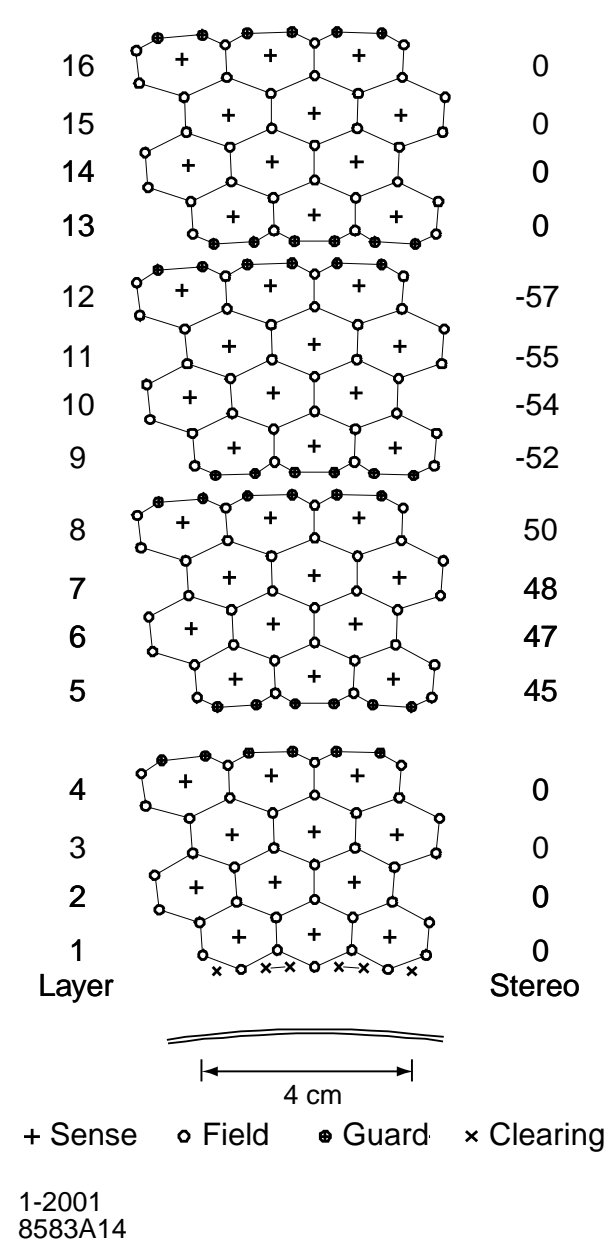

Figure 15: Schematic layout of drift cells for the four innermost superlayers. Lines have been added between field wires to aid in visualization of the cell boundaries. The numbers on the right side give the stereo angles (mrad) of sense wires in each layer.

\subsubsection{DIRC}

The ring-imaging Cherenkov detector utilizes the property of charged particles moving faster than the speed of light in a medium, to emit Cherenkov light. The light is emitted in a cone around the particle direction and the cone angle with respect to the axis, known as Cherenkov angle $\theta_{c}$, depends on the particle velocity $v$ and the medium index of refraction $n, \cos \theta_{c}=1 / n \beta(\beta=v / c, c=$ velocity of light in vacuum). The radiator material of the DIRC is synthetic, fused silica in the 


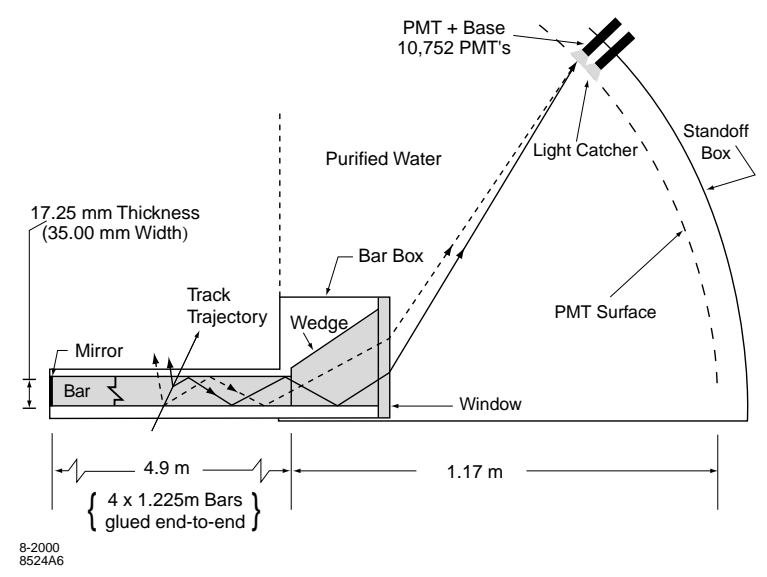

Figure 16: Schematics of the DIRC.

form of 4.9-m long, thin bars with rectangular cross section. The bars are optically isolated with air gaps from one-another and also serve as light pipes transporting the light by total internal reflection, while preserving the Cherenkov angle, to an array of photo-multiplier tubes (PMTs), where the angle is measured, see Fig. 16. Along with the DCH information of the track's angle and momentum, the Cherenkov angle allows to determine the particle velocity and hence identity. It is imperative for the total internal reflection that no condensation occurs on the bars. This is achieved by maintaining a flow of filtered nitrogen gas. The choice of fused silica as the bar material was based on a combination of properties among which resistance to ionizing radiation, large index of refraction, and because it allows excellent optical finish on the surfaces. To avoid instrumenting both ends of the bars with photon detectors and minimize interference with other detector components in the forward region, where particles are preferentially produced, mirrors are placed at the forward ends, while at the back the bars merge into a water-filled tank, called the standoff box. The photons are detected by PMTs arranged radially on the rear tank surface, at about $1.2 \mathrm{~m}$ from the bar end. Purified water is used in the standoff box due to its index of refraction being close to that of fused silica. The distance 


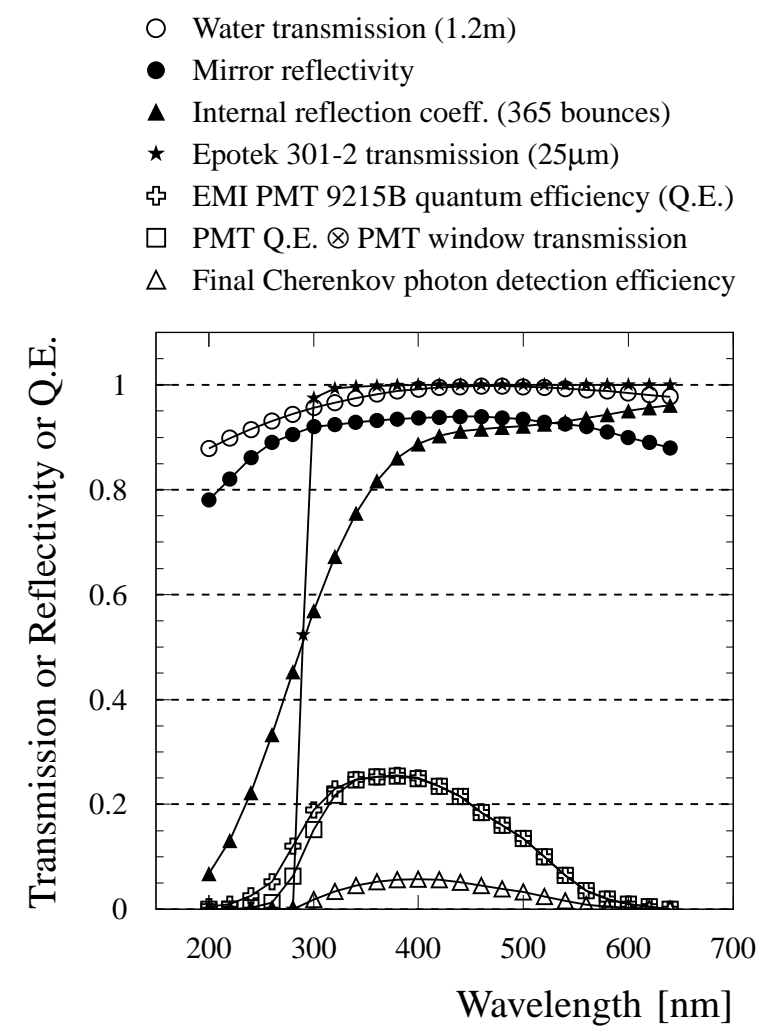

Figure 17: Transmission, reflectivity and quantum efficiency for various components of the DIRC as a function of wavelength for a $\beta=1$ particle at normal incidence to the center of a bar

between the bars and the PMTs along with their sizes gives a Cherenkov angle resolution of about $7 \mathrm{mrad}$. With transmission dispersions included, the overall single photon resolution is estimated to be $\sim 10 \mathrm{mrad}$. The photon detection efficiency of the DIRC depends on the optical properties of the components as well as the PMTs specifics. The wavelength dependent efficiency of some of the components is shown on Fig. 17, where the data points are for particles entering the center of the bar at $90^{\circ}$. In order to maintain good water transparency at wavelengths down to $300 \mathrm{~nm}$, the water must be ultra-pure, de-ionized, de-gassed and free of bacteria. For this purpose several systems are used including filters, a reverse osmosis unit and a UV lamp. Water quality is continuously monitored by checking its resistivity, pH-value, temperature and transparency. For the latter, 
three lasers with wavelengths $266 \mathrm{~nm}, 325 \mathrm{~nm}$ and $442 \mathrm{~nm}$ are used. The entire volume can be recirculated up to four times a day. Potential water seal failure is detected by a dedicated system with a capability of draining the water in 12 minutes.

\subsubsection{EMC}

The electromagnetic calorimeter (EMC) is used for energy and momentum reconstruction of photons as well as electron identification. It must provide excellent energy and angular resolution over a wide energy range. Photons with energies up to $9 \mathrm{GeV}$ need to be measured from QED processes, like $e^{+} e^{-} \rightarrow e^{+} e^{-}(\gamma)$ and $e^{+} e^{-} \rightarrow \gamma \gamma$, for calibration and luminosity determination. On the other hand photons with energies as low as $20 \mathrm{MeV}$ are needed in order to reconstruct $B$-meson decays containing low-energy $\pi^{0} \mathrm{~s}$ and $\eta^{0} \mathrm{~s}$. The requirement on the energy resolution comes from rare $\mathrm{B}$ decays containing $\pi^{0} \mathrm{~s}$ and is on the order of $1-2 \%$, since at energies lower than $2 \mathrm{GeV}$ it dominates the mass resolution of the reconstructed $\pi^{0} \mathrm{~s}$. At higher energies, however, the angular resolution plays a bigger role, which imposes a requirement for the latter to be roughly few mrads. To meet these objectives the EMC consists of appropriately sized thallium-doped cesium iodide $C s I$ crystals arranged projectively with respect to the IP, see Fig. 18. To maximize acceptance in the forward region, a conical endcap is added to the cylindrical barrel extending the polar angle coverage from $15.8^{\circ}$ to $141.8^{\circ}$. The crystals have trapezoidal cross section and increase in length from $29.6 \mathrm{~cm}$ in the backward to $32.4 \mathrm{~cm}$ in the forward direction to fully contain the electromagnetic showers from increasingly higher energy particles. The energy of the electromagnetic showers is measured by reading the scintillation light. For that purpose two silicon PIN diodes 


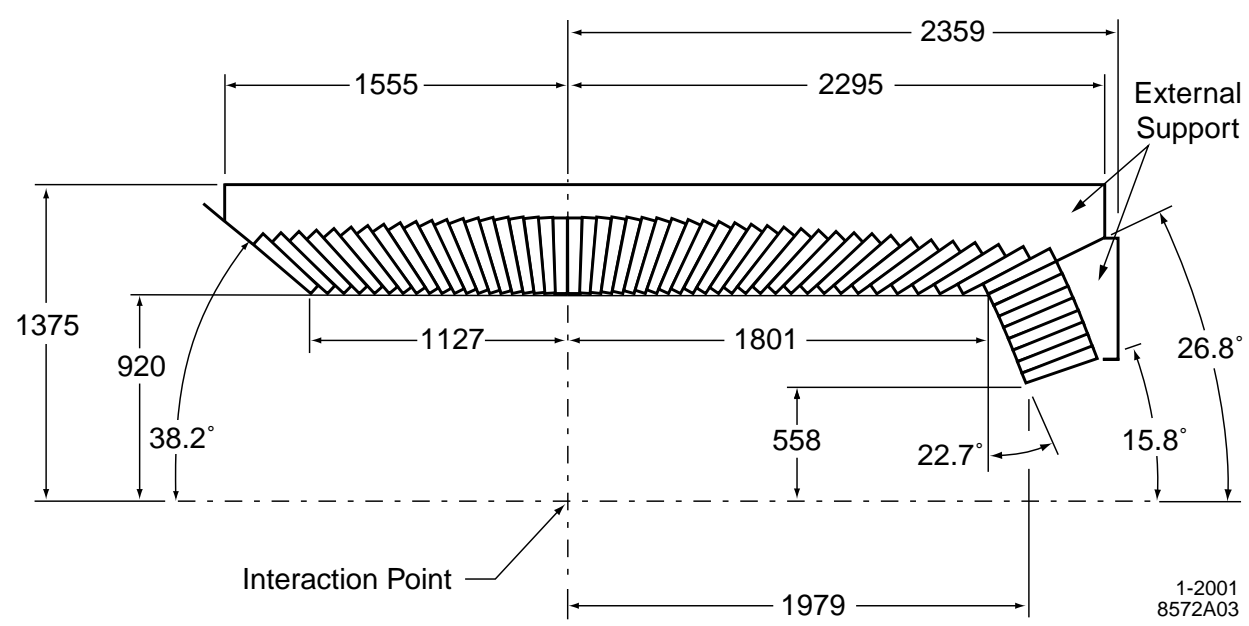

Figure 18: A longitudinal cross section of the EMC (top half). The detector is axially symmetric around the $z$-axis. All dimensions are given in $\mathrm{mm}$.

are glued to the rear face of the crystals and the surrounding area is covered with white reflective paint. The surfaces are polished to facilitate internal reflection as the crystals act not only as a scintillation medium but also as light guides. Transmitted light is recovered in part by wrapping the crystals in defuse white reflectors. In order to achieve uniform light yield along the crystal each was individually measured by placing a highly collimated radioactive source at 20 points along its length. Fine adjustments were made by selectively roughening or polishing the surface to reduce or increase its reflectivity. The crystals were then wrapped in aluminum foil which was grounded to provide Faraday shield and covered with a layer of mylar for electrical isolation. Since the photodiode leakage current depends strongly on the temperature and the diode-crystal joints could experience stress under temperature change due to different termal expansion, an effective cooling system is imperative. The system consists of Fluorinert (polychlorotrifluoro-ethylene) and water chillers for the preamplifiers and the digitizing electronics. The light yield 
of the individual crystals varies significantly and also changes with time under the impact of beam radiation. The front of the crystal is most strongly affected resulting in increased attenuation of the transmitted scintillation light. Individual crystal calibration must therefore be performed at different energies, corresponding to different shower penetration, to compensate the effects of the radiation damage. At $6.12 \mathrm{MeV}$ radioactive photon source provides absolute calibration at lower energies, while at higher energies $(3-9 \mathrm{GeV})$ the relation between polar angle and energy of $e^{ \pm}$from Bhabha events is used. The information obtained from the EMC is processed by reconstruction algorithms which separate the electromagnetic shower, that typically spreads over many adjacent crystals, into clusters and bumps which are associated with a particle. Other algorithms are responsible for reconstructing the angle by employing various weighting schemes on the crystals based on the deposited energy.

\subsubsection{IFR}

The IFR is designed for muon and neutral hadron identification over a large range of momenta and angles. The steel flux return is used as a muon filter and hadron absorber. It is segmented into layers ranging from 2 to $10 \mathrm{~cm}$ in thickness with resistive plate chambers (RPCs) inserted in the gaps between the layers. The RPCs detect streamers from minimum-ionizing particles via two-coordinate capacitance readout strips, see Fig. 19. There are 19 RPC layers in the barrel and 18 in the endcaps. An RPC schematic is shown in Fig. 20. It consists of two bakelite sheets separated by a $2 \mathrm{~mm}$ gap. The external bakelite surfaces are coated with graphite and an $8 \mathrm{kV}$ voltage is applied between them. The signals are red capacitively, on both sides of the gap, by $\mathrm{X}$ and $\mathrm{Y}$ aluminum strips. The 
strips are separated by the ground aluminum plane with a foam sheets. The RPCs operate with a non-flammable gas mixture of argon, Freon and isobutane. The gas is distributed among the chambers at a constant pressure of 6.5 Torr through a parallel manifold system. The IR experimental hall does not have temperature regulation and during the first summer of operation the maximum hall temperature frequently exceeded $31^{\circ} \mathrm{C}$ causing the dark current in many $\mathrm{RPC}$ modules to exceed the capabilities of the high-voltage system. Subsequently water cooling was installed stabilizing the temperature at $20-21^{\circ} \mathrm{C}$ in the barrel, $22^{\circ} \mathrm{C}$ in the backward and $24^{\circ} \mathrm{C}$ in the forward endcaps. During operation at high temperatures many RPCs $(>50 \%)$ showed some efficiency reduction compared to earlier measurements. After the instalation of the cooling system some of them continued to deteriorate, while others remained stable. The cause of the efficiency loss remains under investigation. Recently 24 modules in the endcaps have been replaced by new RPCs. After evaluation of the results with these new RPCs, plans on further IFR improvement can be made.

\subsection{Detector Readout}

The detector readout is achieved with a number of tightly coupled hardware and software systems, see Fig. 21. The front-end electronics for all detector subsystems is mounted on the detector to minimize noise pickup. It performs initial signal amplification and digitization as well as data transfer via optical fibers to the data acquisition system. A two-level trigger is designed to reject beam-induced background. The first level (L1) is implemented in hardware and relies on inputs from the DCH, EMC and IFR. The level 3 (L3) trigger is implemented in software and selects events to be stored for further processing. A provisional second level 


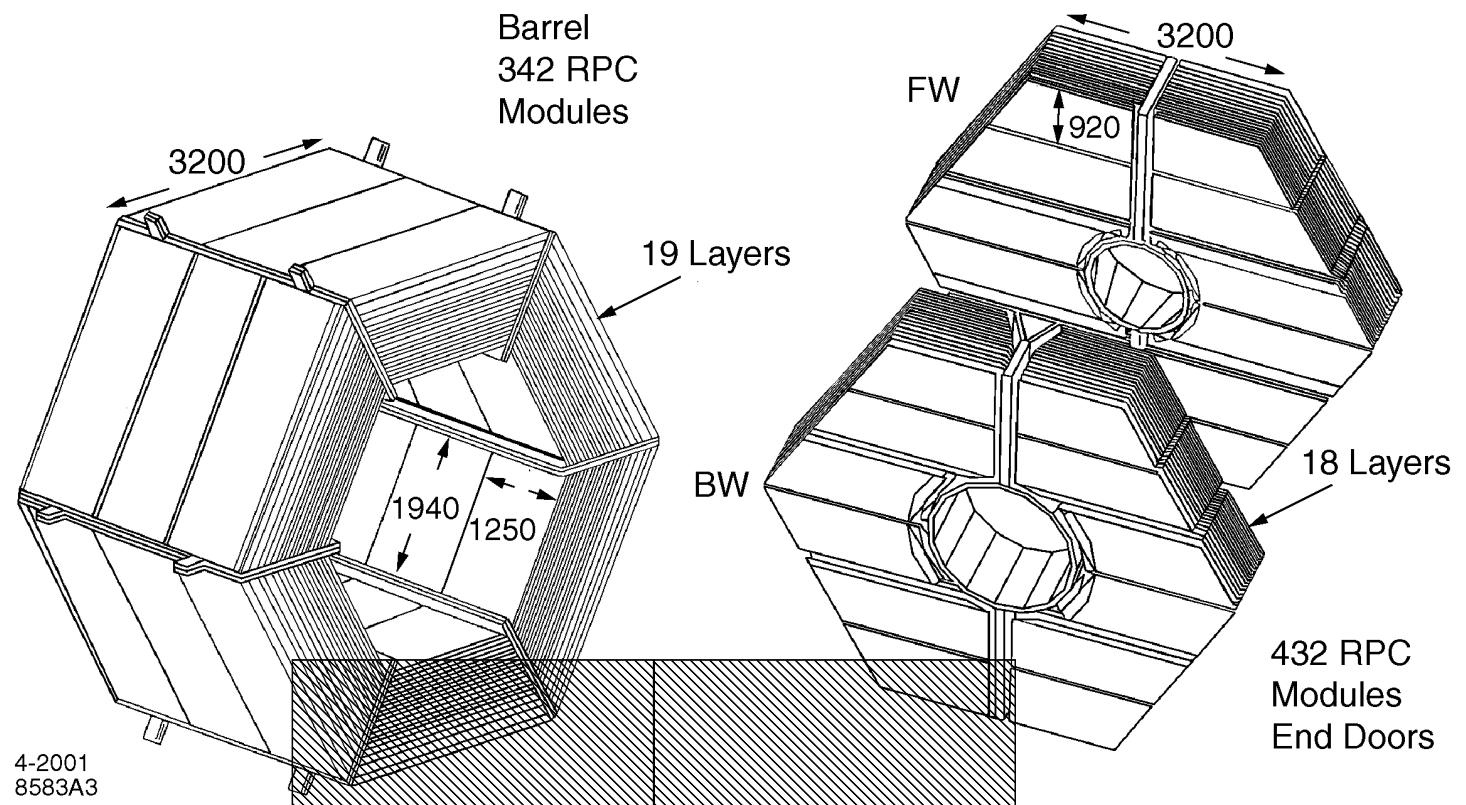

Figure 19: Overview of the IFR: Barrel sectors and forward (FW) and backward (BW) end doors; the shape of the RPC modules and their dimensions are indicated.

trigger is possible should severe conditions require additional sophistication. The online prompt reconstruction (OPR) reads the raw data after L3, selects physics events and writes the results to the event store.

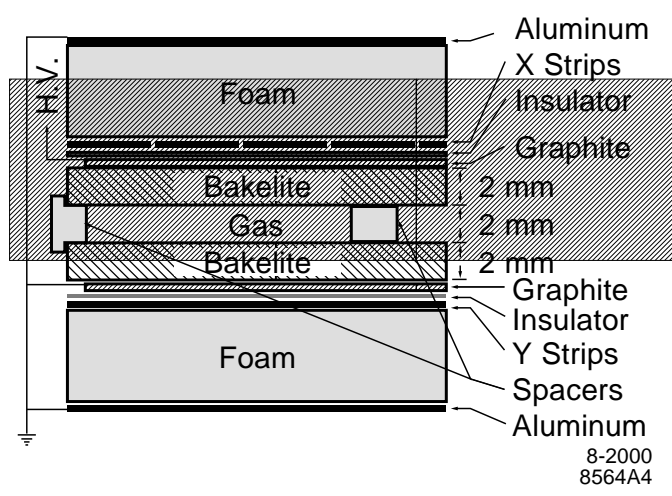

Figure 20: RPC cross section with the schematics of the high voltage connection. 


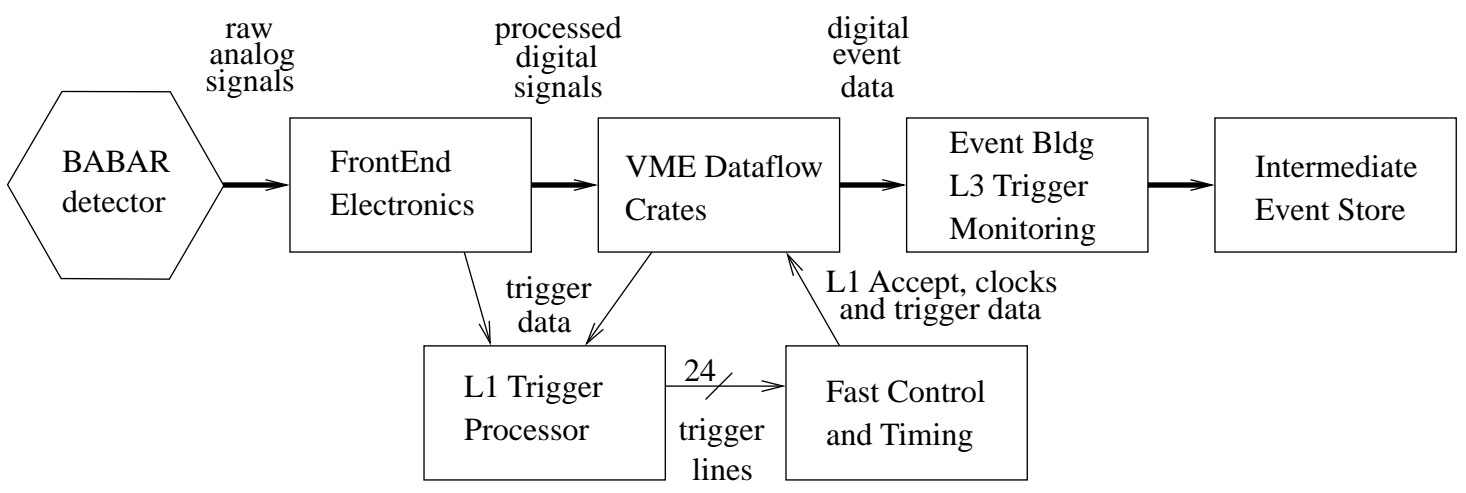

Figure 21: Schematic diagram of the data acquisition. 


\section{H A P T E R 3}

\section{Particle Identification}

In the inclusive $B \rightarrow X_{s} \ell^{+} \ell^{-}$analysis we adopt a sum of exclusive modes

technique. $B$ candidates are reconstructed from an oposite charge $e$ or $\mu$ pair, one $K^{ \pm}$or $K_{s}$ and up to three pions, of which at most one $\pi^{0}$. Thus all particle identification (PID) systems are involved in the analysis and due to the rareness of the process, outstanding performance is necessary. The PID criteria used are outlined in the following sections.

\section{$3.1 \quad$ Electron Id}

For the electrons we use the Likelihood Selector. A standard very loose set of cuts is applied to all charged tracks in the event:

- $0.5<E / p<5$, where E and $\mathrm{p}$ are the EMC-measured energy and the DCHmeasured momentum of the associated track, respectively.

- Number of crystals associated with the track $>3$

- $500<d E / d x$ ( the ionizing energy loss in $\mathrm{DCH})<1000$

A likelihood $L$ for each particle hypothesis is then calculated based on the variables: 
- $E / p$

- Lateral moment of the EMC cluster: $\mathrm{LAT}=\frac{R M}{R M+25\left(E_{1}+E_{2}\right)}$. Here $E_{1}$ and $E_{2}$ are the energies of the two most energetic crystals and $R M=\sum\left(r_{i} / 1.08\right)^{2} E_{i}$, where the sum goes over all but the two most energetic crystals and $r$ is the distance between a point located $12.5 \mathrm{~cm}$ from the crystal surface along its axis and the line connecting the cluster centroid (also $12.5 \mathrm{~cm}$ from the surface) and the origin of the coordinate system. For clusters with less than three crystals LAT is 0 .

- $\triangle \phi=$ charge $\left(\phi_{A t E M C}-\phi_{\text {cluster }}\right)$, where $\phi_{A t E M C}$ is the $\phi$ coordinate of the track intersection point with the EMC and $\phi_{\text {cluster }}$ is the $\phi$ coordinate of the cluster centroid projected to the crystal face.

- $d E / d x(\mathrm{DCH})$

- The Cherenkov angle from DIRC: $\theta_{C}$

A likelihood fraction is defined as

$$
f=\frac{P_{e} L(e)}{P_{e} L(e)+P_{\pi} L(\pi)+P_{K} L(K)+P_{p} L(p)},
$$

where $P_{e}$ and $P_{K}$ are equal to unity, $P_{\pi}=5$ and $P_{p}=0.2$. These values are prompted by the average multiplicity of these particles in an event. Electron candidates are selected by using the default tight selection corresponding to a cut of $f>0.95$. Plots of the efficiency and the pion missid rate as a function of the momentum are shown on Figs. 22 and 23. 


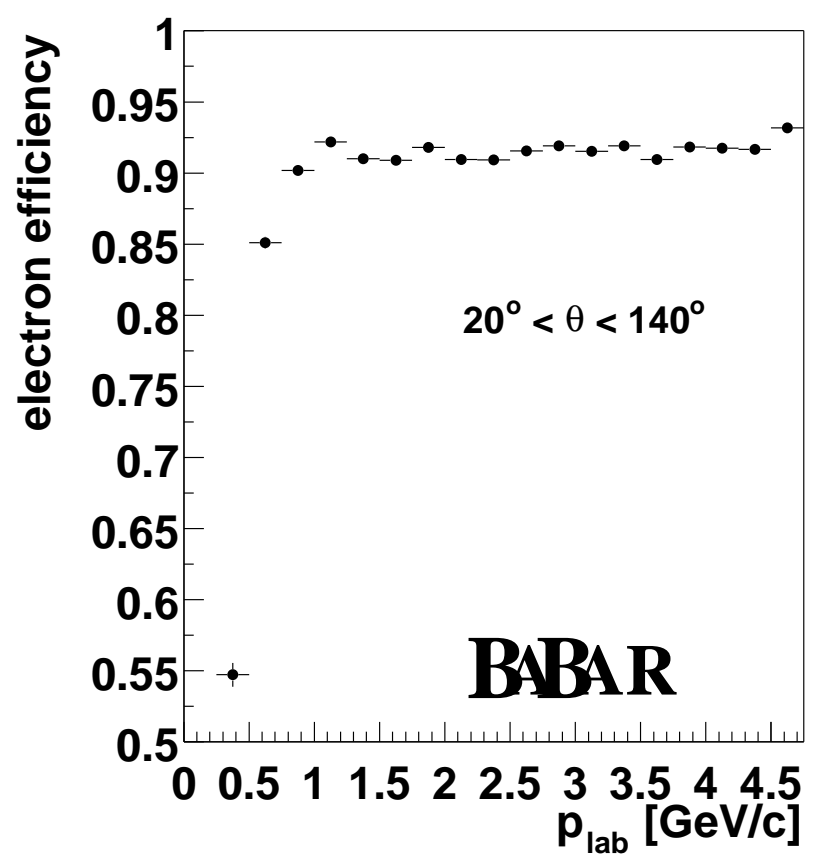

Figure 22: Electron Id efficiency vs momentum.

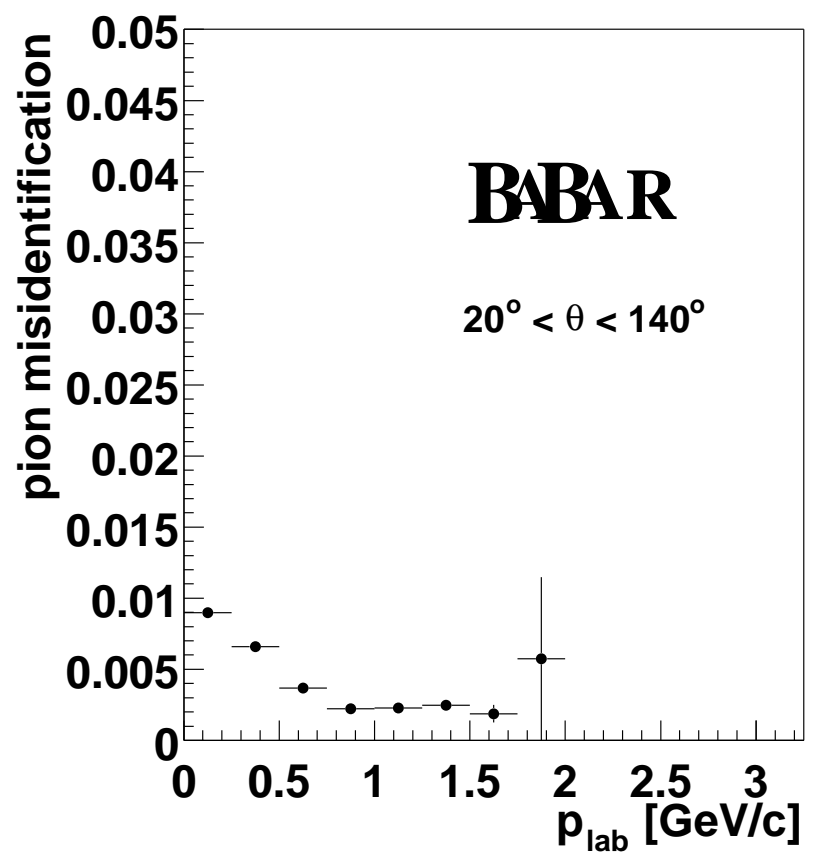

Figure 23: Pion miss-Id vs momentum. 


\subsection{Muon Id}

The muons are identified with the following variables:

1. Calorimeter energy: $E_{c a l}$

2. Number of IFR layers hit: $N_{L}$

3. A boolean variable which is true when a cluster has a hit in the inner RPC: $I_{h}$

4. The first IFR hit layer in the cluster: $F_{h}$

5. The last IFR hit layer in the cluster: $L_{h}$

6. The number of interaction lengths traversed by the track in the detector: $\lambda$

7. The number of interaction lengths in the detector which a muon with the same momentum is expected to traverse: $\lambda_{\text {exp }}$

8. The $\chi^{2}$ of the IFR cluster hits with the extrapolated track from DCH: $\chi_{\text {trk }}^{2}$

9. The $\chi^{2}$ of the IFR track fit: $\chi_{\text {fit }}^{2}$

10. The average multiplicity of hit strips per layer: $\bar{m}$

11. The standard deviation of $\bar{m}: \sigma_{\bar{m}}$

We use the very tight selector corresponding to cuts:

- $0.05<E_{\text {cal }}<0.4$

- $N_{L} \geq 2$

- $\triangle \lambda=\lambda_{\text {exp }}-\lambda<0.8$ 
- $\lambda>2.2$

- $\chi_{t r k}^{2}<5$

- $\chi_{\text {fit }}^{2}<3$

- $T_{c}=\left[\begin{array}{c}\frac{N_{L}}{L_{h}-F_{h}+1} \text { if } \mathrm{I}_{\mathrm{h}}=\text { false } \\ \frac{N_{L}}{L_{h}-F_{h}} \text { if } \mathrm{I}_{\mathrm{h}}=\text { true }\end{array}\right]>0.34$

- $\bar{m}<8$

- $\sigma_{\bar{m}}<4$

Tha plateau efficiency, starting from around $2 \mathrm{GeV}$, for the very tight selector is about $70.3 \%$ with missidentification rate of $2.3 \%$.

\subsection{Charged Kaon Id}

Charged kaons are identified with the PidKaonMicroSelector. The selector uses a neural network, taking inputs from the SVT, the DCH and the DIRC. These inputs are:

- The momentum of the candidate: $p$

- $\frac{L_{K}^{S V T}}{L_{K}^{S V T}+L_{\pi}^{S V T}}$

- $\frac{L_{K}^{D C H}}{L_{K}^{D C H}+L_{\pi}^{D C H}}$

- $\frac{L_{K}^{D I R C}}{L_{K}^{D I R C}+L_{\pi}^{D I R C}}$

where $L_{K}^{\text {system }}$ and $L_{\pi}^{\text {system }}$ are likelihoods for $K$ and $\pi$ hypothesis, respectively, for the corresponding detector system. In this analysis we use the KMicroLoose selection, corresponding to a cut of 0.5 on the neural network output. The efficiency 
and pion miss-Id for the KMicroLoose selector are shown in Figs. 24 and 25, the red and blue dots correspond to an older and newer code release version. 


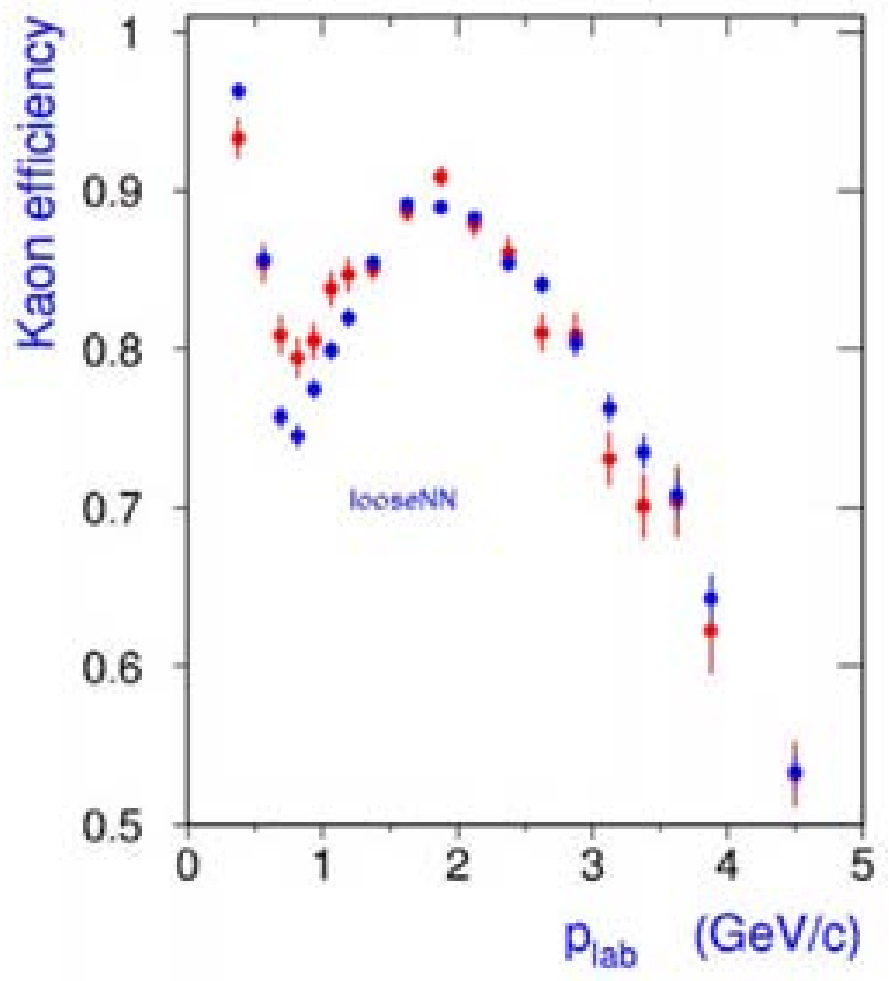

Figure 24: Kaon Id efficiency vs momentum.

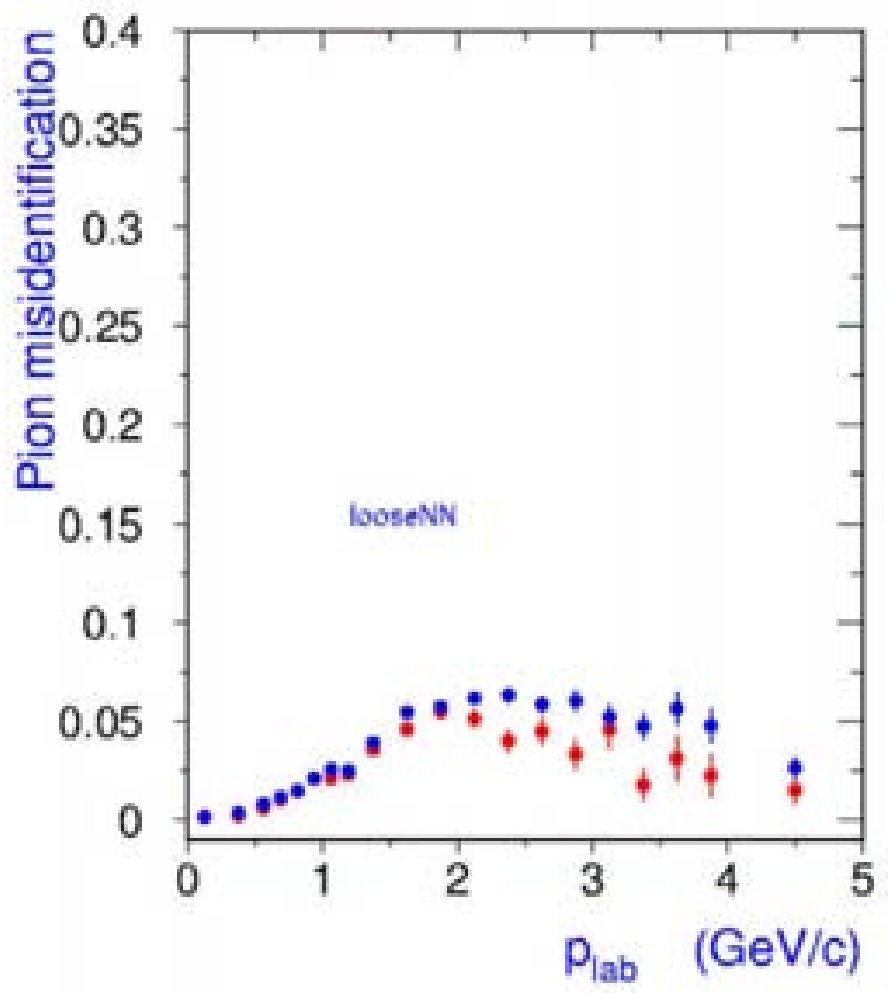

Figure 25: Pion miss-Id vs momentum. 


\subsection{Neutral Kaon Id}

We only consider Ks decaying into two charged pions. The candidates are taken from the KsDefault list and are made out of oppositely charged tracks with fitted mass within $0.025 \mathrm{GeV}$ of the PDG Ks mass, $497.7 \mathrm{MeV}$. In order to purify the sample three additional cuts are applied:

- A tighter cut on the reconstructed Ks mass: $|m(\pi \pi)-m(K s)|<11.2 \mathrm{MeV}$

- $L_{d e c}>2 \mathrm{~mm}$, where $L_{d e c}$ is the decay length calculated as the distance between the Ks decay point and the event primary vertex

- $\cos \alpha>0.99$, where $\alpha$ is the angle between the Ks momentum and the primary vertex - decay point line

\subsection{Charged Pion Id}

For the charged pions we use particles from the GoodTracksLoose list which are not consistent with being KMicroVeryTight and eMicroVeryTight. The pion candidates in the GoodTracksLoose list satisfy the following requirements:

- Min transverse momentum: $0.1 \mathrm{GeV}$

- Max momentum: $10 \mathrm{GeV}$

- Min number of DCH hits: 12

- Max distance of closest approach (DOCA) in XY plane: $1.5 \mathrm{~cm}$

- Min Z DOCA: $-10 \mathrm{~cm}$ 
- Max Z DOCA: $10 \mathrm{~cm}$

The kaon candidates in the KMicroVeryTight list correspond to a cut of 0.68 on the neural network output described in Sec. 3.3. The eMicroVeryTight lists consists of candidates which satisfy the requirements:

- $540<d E / d x<860$

- EMC shower of at least 3 crystals

- $0.89<E / p<1.2$

- $0.1<L A T<0.6$

- $-10<A_{42}<0.11$, where $A_{42}$ is the modulus of the Zernike moment of order $(4,2)[21]$.

- At least three photons detected in the DIRC and Cherenkov angle consistent with electron hypothesis within $3 \sigma$

- DCH track matched to the EMC cluster

\subsection{Neutral Pion Id}

The $\pi^{0}$ particles are composed of photon candidates identified by EMC bumps unmatched to any track. The bumps must have $L A T<0.8$ and energy $E>0.05$ $\mathrm{GeV}$. In order to compute the photons 3-momenta their origin is assumed to be at the primary event vertex. In addition we apply cuts:

- $E\left(\pi^{0}\right)>0.4 \mathrm{GeV}$

- $|m(\gamma \gamma)-m(\pi 0)|<10 \mathrm{MeV}$ 


\section{H A P T E R 4}

\section{Analysis overview}

Our aim is to measure the overall inclusive branching fraction for the $B \rightarrow$ $X_{s} \ell^{+} \ell^{-}$decay, considering electron and muon modes, as well as the branching fractions in several dilepton and hadronic mass regions. The inclusive branching fraction measurement for the electron modes is done for $m_{e^{+} e^{-}}>0.2 \mathrm{GeV}$. The results we present are based on a sample of $81.9 \mathrm{fb}^{-1}$ of on-resonance data. We also used $9.6 \mathrm{fb}^{-1}$ of off-resonance data for continuum background studies.

The sum of exclusive modes technique we have adopted allows us to use the strong background suppression power of the two kinematic variables $\Delta E$ and $m_{\mathrm{ES}}$ described in Section 2.1. However, it also introduces a significant hadronization model dependence, since the signal reconstruction efficiency varies with the particle multiplicity of the hadronic system $\left(X_{s}\right)$ in the final state.

\subsection{Monte Carlo generator}

We use Monte Carlo (MC) generated signal and background events to estimate the final signal reconstruction efficiency, tune the background suppression cuts and asses the systematic errors. The overall signal model is a combination of three separate Monte Carlo generators: $B \rightarrow K^{+} \ell^{-}, B \rightarrow K^{*} \ell^{+} \ell^{-}$for $m_{X s}<1.1 \mathrm{GeV}$ 
and $b \rightarrow s \ell^{+} \ell^{-}$for $m_{X s} \geq 1.1 \mathrm{GeV}$. The exclusive $B \rightarrow K \ell^{+} \ell^{-}$and $B \rightarrow K^{*} \ell^{+} \ell^{-}$ decays are generated according to the model by Ali, Ball, Handoki and Hiller [3], where the required form factors are computed using light cone QCD sum rules. For the inclusive $b \rightarrow s \ell^{+} \ell^{-}$decays we set the spectator-quark mass $m_{q}$ to zero and the Fermi motion parameter $p_{F}$ to $410 \mathrm{MeV}$, as suggested by the CLEO $B \rightarrow X_{s} \gamma$ data [22]. All three generators use NNLO Wilson coefficients, following Ali et al. [1]. The three pieces are combined according to the branching fraction predictions for $m_{l l}>0.2 \mathrm{GeV}$, see end of Sec. 1.3.2, multiplied by a factor reflecting the difference between the lifetimes of the $B^{+}$and $B^{0}$ mesons: $\tau\left(B^{+}\right) / \tau\left(B^{0}\right)=1.083 \pm 0.017$ [2]. Since the exclusive predictions in Table 2 are given for $B^{0}$ decays, we multiply the corresponding branching fractions for $B^{+}$decays by 1.083 . The predictions for the inclusive decays, however, are averaged over charged and neutral $B$ mesons, thus we multiply the branching fraction for $b \rightarrow s \ell^{+} \ell^{-}$by 1.04 for charged $B$ mesons and 0.96 for neutral $B$ mesons.

Figures 26 through 29 show the hadronic and dilepton mass distributions for the combined MC signal model in the electron and muon channels. The fractions of final states in different hadronic topologies are given in Table 3.

The various signal and background MC samples used in this analysis are summarized in Table 4. 


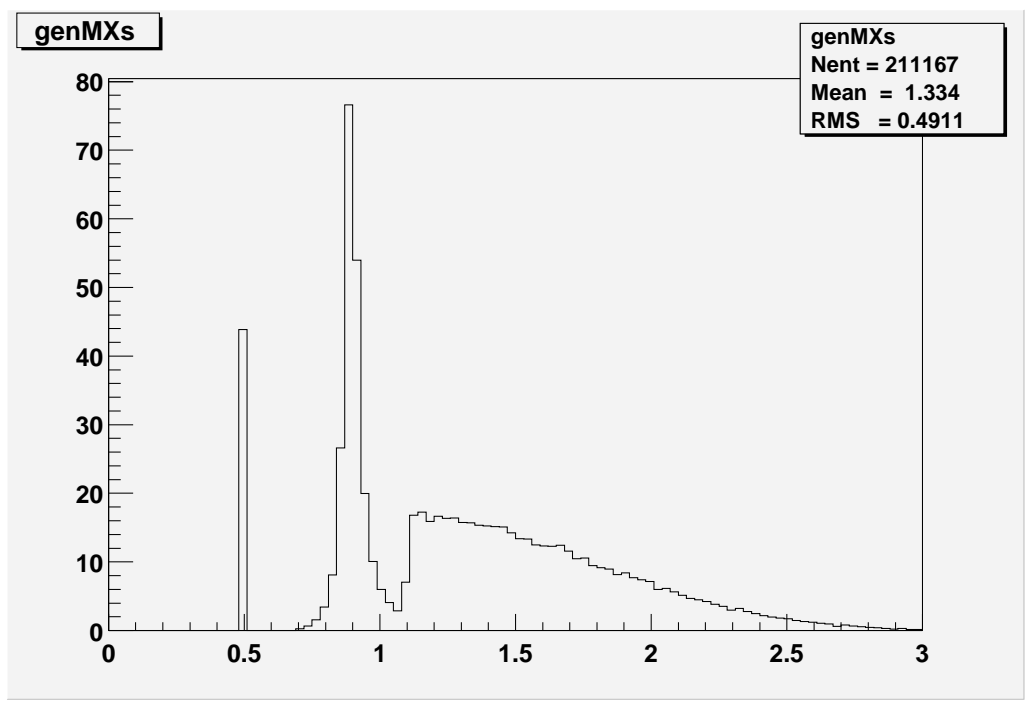

Figure 26: Hadronic mass distribution of the signal model in the electron channel. The entries are normalized to the expected number in 81.9 $\mathrm{fb}^{-1}$.

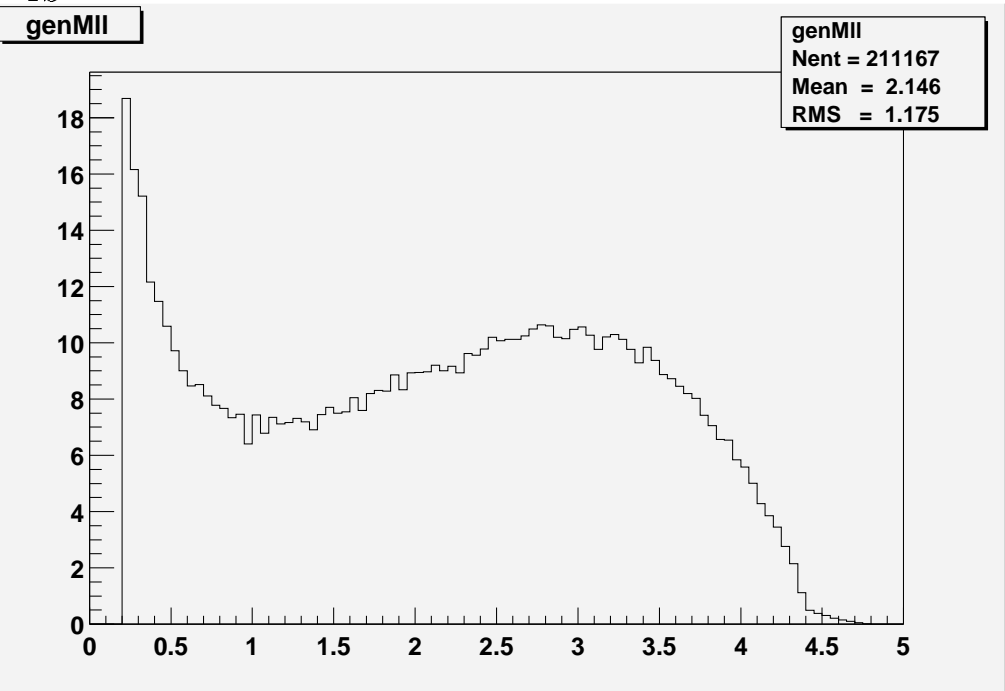

Figure 27: Dilepton mass distribution of the signal model in the electron channel for $m_{l l}>0.2 \mathrm{GeV}$. The entries are normalized to the expected number in $81.9 \mathrm{fb}^{-1}$. 


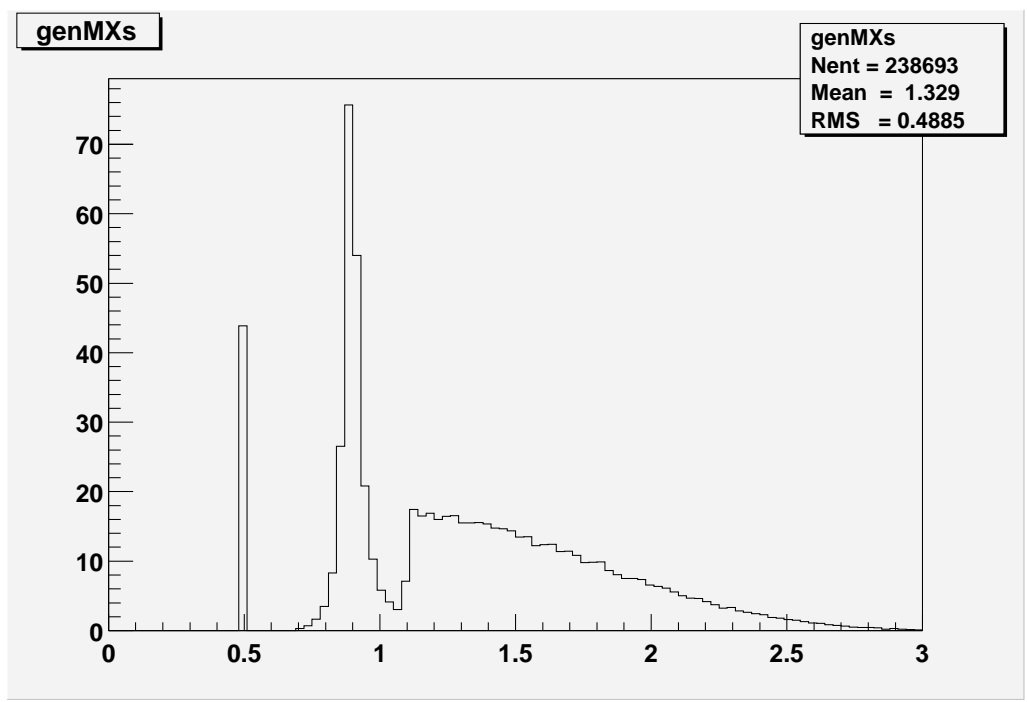

Figure 28: Hadronic mass distribution of the signal model in the muon channel. The entries are normalized to the expected number in $81.9 \mathrm{fb}^{-1}$.

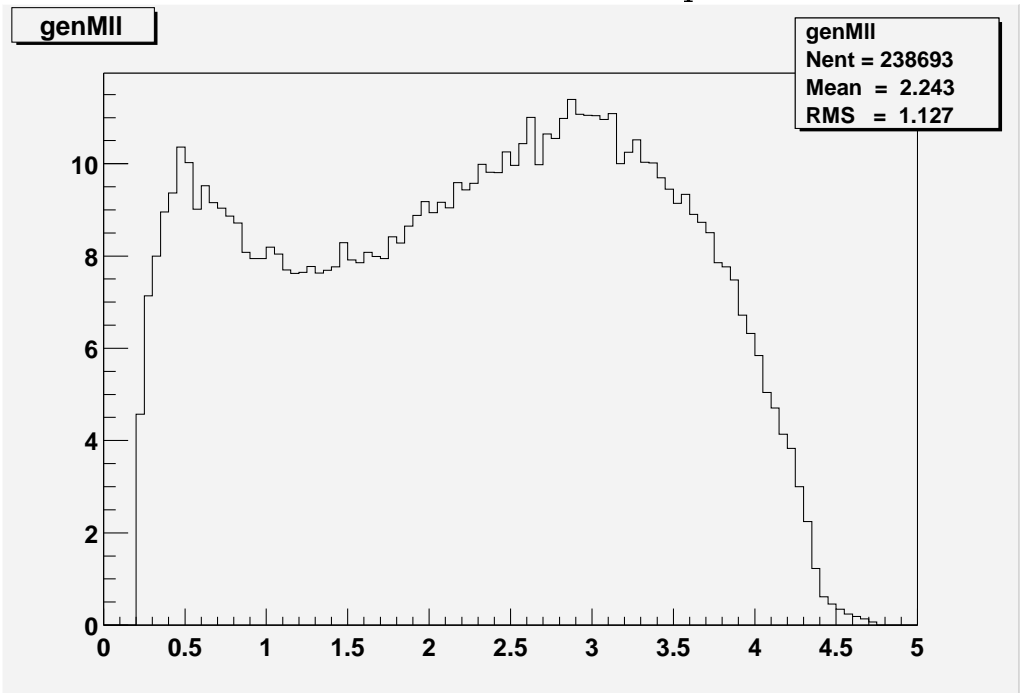

Figure 29: Dilepton mass distribution of the signal model in the muon channel. The entries are normalized to the expected number in $81.9 \mathrm{fb}^{-1}$. 
Table 3: Fractions of $B$ decays in different hadronic final states for the signal model.

\begin{tabular}{ccc}
\hline \hline$X_{s}$ topology & $B \rightarrow X_{s} e^{+} e^{-}\left(\right.$with $\left.m_{l l}>0.2 \mathrm{GeV}\right)$ & $B \rightarrow X_{s} \mu^{+} \mu^{-}$ \\
\hline$K$ & 0.044 & 0.044 \\
$K \pi^{0}$ & 0.071 & 0.072 \\
$K \pi$ & 0.125 & 0.126 \\
$K \pi \pi^{0}$ & 0.056 & 0.053 \\
$K \pi \pi$ & 0.049 & 0.051 \\
$K \pi \pi \pi^{0}$ & 0.036 & 0.036 \\
$K \pi \pi \pi$ & 0.010 & 0.010 \\
\hline$K_{s}$ & 0.021 & 0.021 \\
$K_{s} \pi^{0}$ & 0.032 & 0.032 \\
$K_{s} \pi$ & 0.069 & 0.069 \\
$K_{s} \pi \pi^{0}$ & 0.031 & 0.030 \\
$K_{s} \pi \pi$ & 0.023 & 0.022 \\
$K_{s} \pi \pi \pi^{0}$ & 0.015 & 0.016 \\
$K_{s} \pi \pi \pi$ & 0.006 & 0.006 \\
\hline
\end{tabular}

\subsection{Analysis roadmap}

The analysis consists of several stages, which are outlined below. The procedure is motivated by the fact that the signal yield is extremely small, which necessitates background suppression better than $1 / 10^{7}$ in order to obtain a final signal-to-background ratio of about $1 / 1$.

1. Events are required to have a good primary vertex and pass a loose skim (see Sec. 5.1) intended to select hadronic events with at least two charged leptons.

2. In each event $B$ candidates are formed by first picking a lepton pair, and then adding one $K^{ \pm}$or $K_{s}$ and up to three pions, of which at most one can be a $\pi^{0}$. In this way fourteen different modes are considered: $\mathrm{K}, \mathrm{K} \pi^{0}, \mathrm{~K} \pi, \mathrm{K} \pi \pi^{0}$, $\mathrm{K} \pi \pi, \mathrm{K} \pi \pi \pi^{0}, \mathrm{~K} \pi \pi \pi, K_{s}, K_{s} \pi^{0}, K_{s} \pi, K_{s} \pi \pi^{0}, K_{s} \pi \pi, K_{s} \pi \pi \pi^{0}, K_{s} \pi \pi \pi$.

3. The number of $B$ candidates per event is reduced by applying a set of loose 
Table 4: Monte Carlo samples used in the analysis.

\begin{tabular}{lr}
\hline \hline Sample & \# events \\
\hline$B \rightarrow K^{+} e^{+} e^{-}$ & 54000 \\
$B \rightarrow K_{s} e^{+} e^{-}$ & 51000 \\
$B \rightarrow K^{*} e^{+} e^{-}$with $m_{X s}<1.1 \mathrm{GeV}$ & 46828 \\
$B \rightarrow X_{s} e^{+} e^{-}$with $m_{X s} \geq 1.1 \mathrm{GeV}$ & 59658 \\
\hline$B \rightarrow K^{+} \mu^{+} \mu^{-}$ & 51000 \\
$B \rightarrow K_{s} \mu^{+} \mu^{-}$ & 52000 \\
$B \rightarrow K^{*} \mu^{+} \mu^{-}$with $m_{X s}<1.1 \mathrm{GeV}$ & 49703 \\
$B \rightarrow X_{s} \mu^{+} \mu^{-}$with $m_{X s} \geq 1.1 \mathrm{GeV}$ & 52597 \\
\hline$B \rightarrow J / \psi X$ & 390000 \\
$B \rightarrow \psi(2 S) X$ & 137000 \\
\hline Generic $u d s$ & $96.3 \times 10^{6}$ \\
Generic $c \bar{c}$ & $60.7 \times 10^{6}$ \\
Generic $B^{+} B^{-}$ & $75.2 \times 10^{6}$ \\
Generic $B^{0} \bar{B}^{0}$ & $75.1 \times 10^{6}$ \\
\hline
\end{tabular}

preselection cuts. A signal likelihood is then calculated for each candidate passing the preselection to pick the best candidate in the event.

4. The best candidate is subjected to a series of background rejection postselection cuts. These include cuts on the dilepton invariant mass to suppress backgrounds from $B \rightarrow J / \psi X_{s}, B \rightarrow \psi(2 S) X_{s}$ and $B \rightarrow X_{s} \gamma$ with $\gamma \rightarrow e^{+} e^{-}$, which are peaking in $m_{E S}$, as well as cuts reducing the combinatorial background from $B \bar{B}$ and continuum events.

5. The signal yield is extracted with an extended unbinned maximum likelihood fit to the $m_{E S}$ distribution. 


\section{H A P T E R 5}

\section{Signal reconstruction and background suppression}

\subsection{Event selection}

In the first selection stage, events are required to have a good primary vertex (converging fit) and pass the following skim criteria, generally satisfied by multihadron, but not by continuum events:

1. The number of charged tracks is greater than three.

2. The ratio between the second and zeroth order Fox-Wolfram moments [23], $R_{2}$, calculated using charged tracks and neutral calorimeter clusters must be less than 0.5 . The value of $R_{2}$ ranges from 0 to 1 and is indicative of the jettiness of the event. Values close to 1 indicate a jetty event, while values closer to 0 correspond to a more spherical event.

3. Either the $\mathrm{DCH}$ or the EMC L3 trigger fired to guarantee an event triggered by an electron positron collision rather than a cosmic event.

4. The event contains at least two leptons, which could be either electrons or muons satisfying loose particle identification criteria and lab frame momenta greater than $0.5 \mathrm{GeV}$ and $0.8 \mathrm{GeV}$, respectively. 
The efficiency for the above requirements is $95 \%$ for both $B \rightarrow X_{s} e^{+} e^{-}$and $B \rightarrow$ $X_{s} \mu^{+} \mu^{-}$signal MC.

\section{2 $B$ candidate selection}

In the second selection stage, the $e^{+} e^{-}$or $\mu^{+} \mu^{-}$pair, if any, with the highest lab frame energy and converging vertex is picked. The signal efficiency for this stage is $93.5 \%$ for the electron and $84.8 \%$ for the muon modes. Because of their relatively low mass, the electrons are likely to loose significant amount of their energy via Bremsstrahlung. We perform a Bremsstrahlung recovery by picking up to three photons lying within a small angular region around the electron direction to calculate the electron energy. For vertexing we use the original non-recovered electrons. Using only the highest energy dilepton, numerous $B \rightarrow X_{s} \ell^{+} \ell^{-}$candidates are formed in every event by adding a $K$ or a $K_{s}$, and up to three pions (of which at most one $\pi^{0}$ ). A candidate is rejected if it fails any of the following preselection requirements:

1. The dilepton vertex fit probability $\left(P_{\ell \ell v t x}\right)$ satisfies $\log \left(P_{\ell \ell v t x}\right)>-10$.

2. The $B$ vertex fit probability $\left(P_{B v t x}\right)$, using only the charged particles, satisfies $\log \left(P_{B v t x}\right)>-10$.

3. The invariant mass of the hadronic system $m_{X s}<2.5 \mathrm{GeV}$.

4. The beam energy-substituted mass, see Sec. 2.1 , satisfies $5.00<m_{E S}<5.29$ $\mathrm{GeV}$.

5. The difference between the energy of the $B$ candidate and the beam energy in the CM frame $|\Delta E|<0.3 \mathrm{GeV}$. 
These criteria retain $77 \%$ and $90 \%$ of the electron and muon MC signal decays, respectively. The best candidate in the event is selected among the ones passing the preselection. In an average MC signal event there are about 5 candidates to choose from with some events having up to 30, see Figs. 30 and 31, the figures also show distributions of the number of combinations per event for $B \bar{B}$, continuum $\mathrm{MC}$ and data. In events with two or more candidates, the one with the highest signal likelihood is selected. The likelihood function is based on distributions of the variables $\Delta E, P_{B v t x}$ and $\cos \theta_{B}$, where $\theta_{B}$ is the angle between the momentum of the $B$ candidate and the beam axis in the CM frame. Figures 32 and 33 show the distributions of these variables for truth-matched (correctly reconstructed) signal decays and all other decays from the signal MC sample, hereby referred to as crossfeed decays. The $\Delta E$ distributions for the truth-matched signal exhibit a radiative tail, which is more pronounced for the electron modes. Since the initial electron and positron are relativistic, the $\Upsilon(4 S)$ is created with its spin aligned parallel to the $z$ direction. Thus the two scalar $B$ mesons are preferentially produced in perpendicular directions to the $z$ axis in the $\mathrm{CM}$ frame to conserve the angular momentum, resulting in the characteristic $\cos \theta_{B}$ distribution for the truth-matched signal. A cross-feed event would typically miss a signal decay product and/or include a particle from the other $B$. Such events tend to have flatter $\Delta E$ and $\cos \theta_{B}$ distributions and a lower vertex quality (if a charged particle from the other $B$ was included).

The probability density functions (PDFs) for $\Delta E, \log \left(P_{B v t x}\right)$ and $\cos \theta_{B}$ are the sum of a Novosibirsk and a Gaussian function, the sum of a first-order polynomial and an exponential, and a second order polynomial, respectively. The signal likelihood is the product of these one-dimensional PDFs. The Novosibirsk function is 

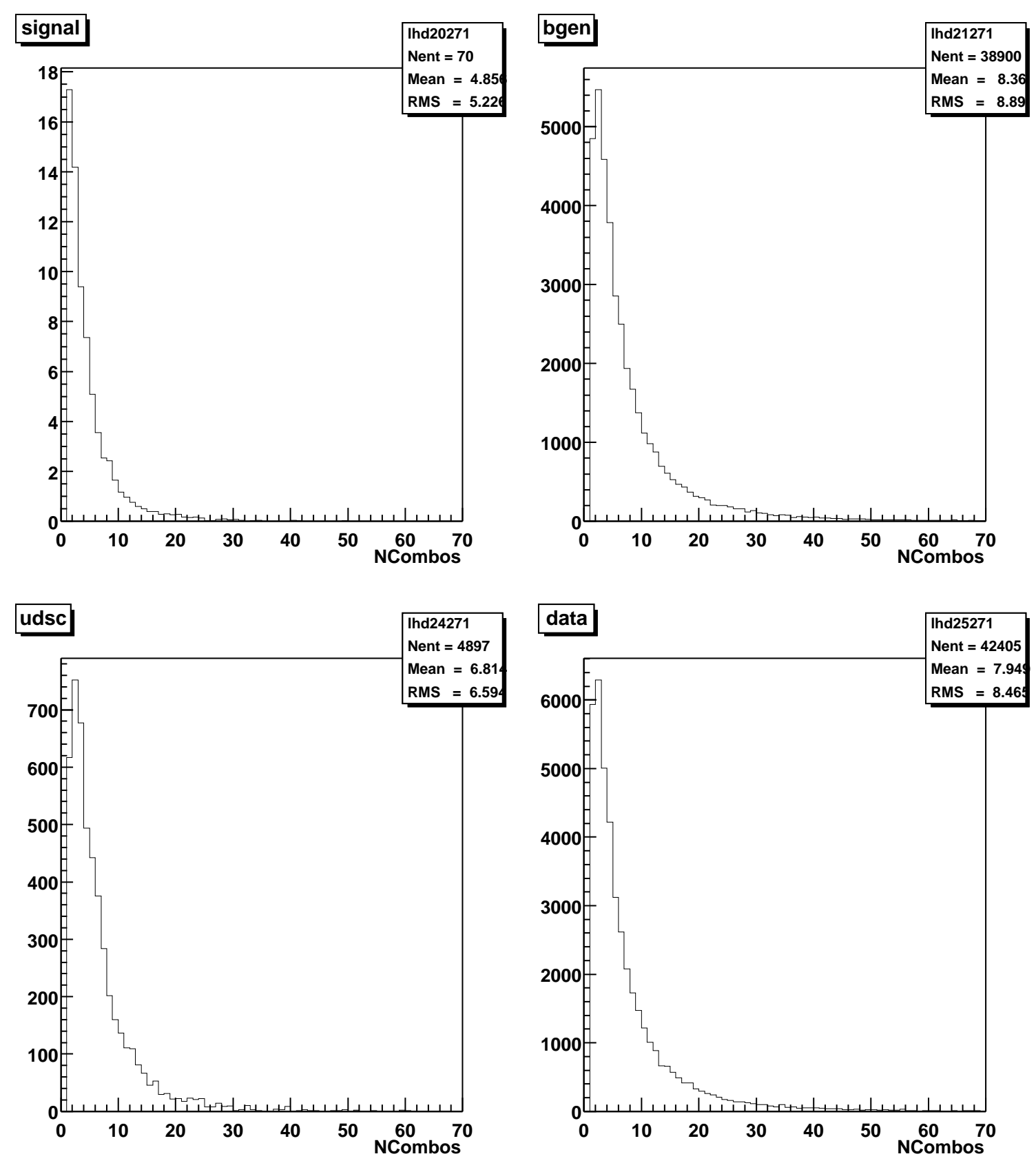

Figure 30: Distributions of the number of $B \rightarrow X_{s} e^{+} e^{-}$candidates per event after preselection for $B \rightarrow X_{s} \ell^{+} \ell^{-}$(top left), $B \bar{B}$ (top right), continuum MC (bottom left), and data events (bottom right). 

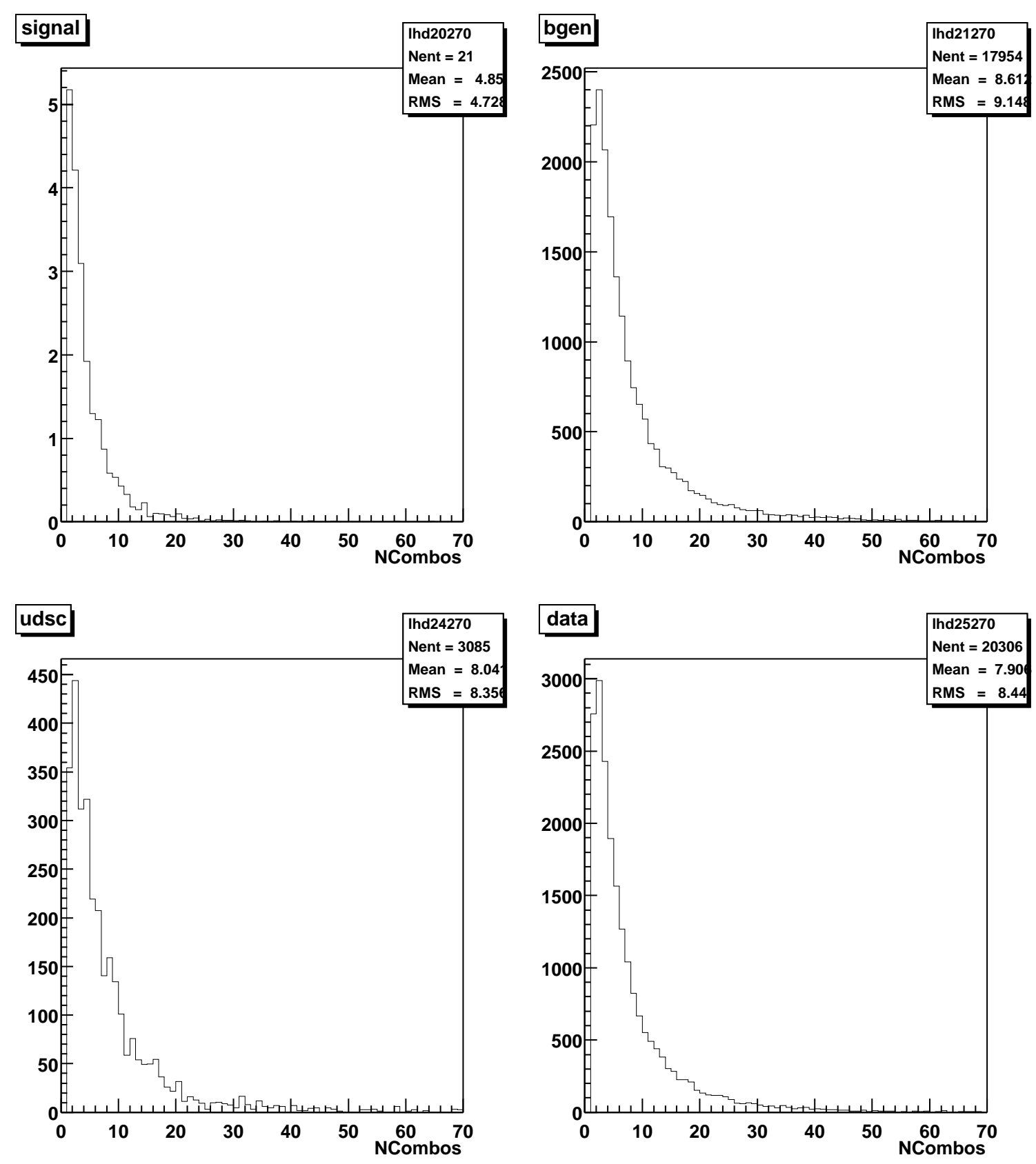

Figure 31: Distributions of the number of $B \rightarrow X_{s} \mu^{+} \mu^{-}$candidates per event after preselection for $B \rightarrow X_{s} \ell^{+} \ell^{-}$(top left), $B \bar{B}$ (top right), continuum MC (bottom left), and data events (bottom right). 

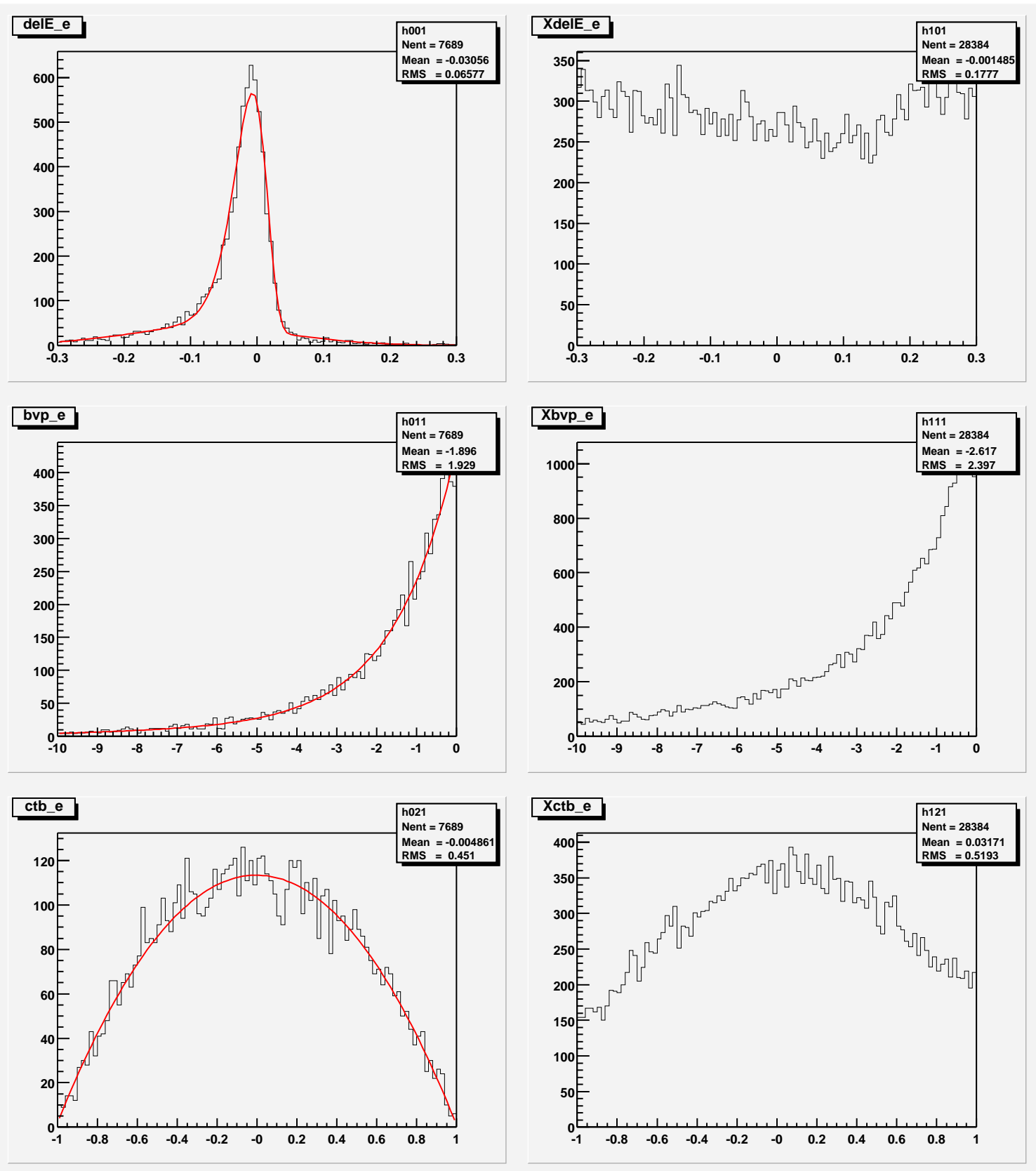

Figure 32: Distributions of $\Delta E$ (top), $\log \left(P_{B v t x}\right)$ (middle), and $\cos \theta_{B}$ (bottom) for truth-matched $B \rightarrow X_{s} e^{+} e^{-}$decays (left column), and all other decays (right column), a.k.a. cross-feed, in the signal $B \rightarrow X_{s} \ell^{+} \ell^{-}$ MC. 

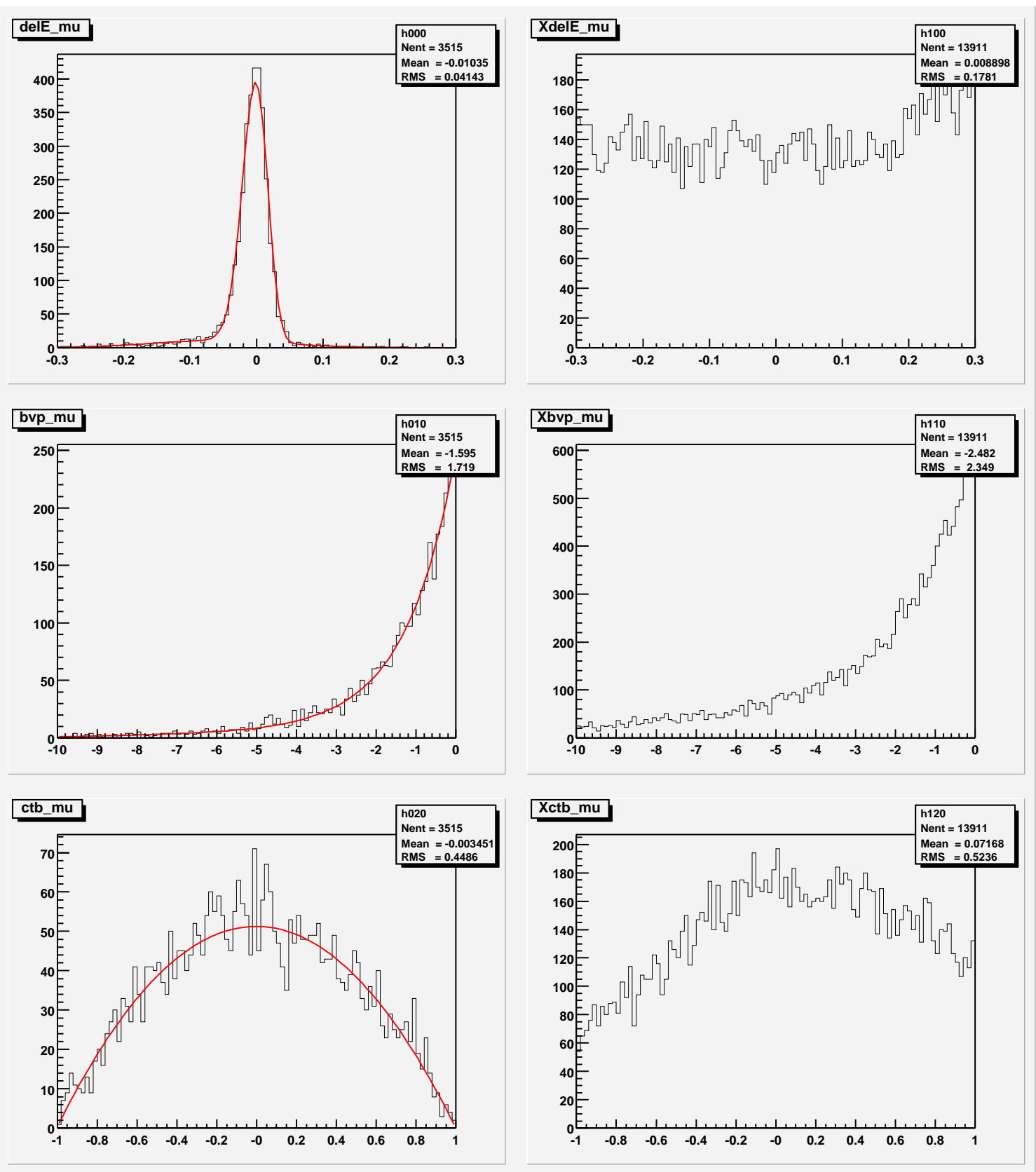

Figure 33: Distributions of $\Delta E$ (top), $\log \left(P_{B v t x}\right)$ (middle), and $\cos \theta_{B}$ (bottom) for truth-matched $B \rightarrow X_{s} \mu^{+} \mu^{-}$decays (left column), and all other decays (right column), a.k.a. cross-feed, in the signal $B \rightarrow X_{s} \ell^{+} \ell^{-}$ MC. 
effectively a Gaussian with an asymmetric tail

$$
f_{\text {Novo }}(x, \mu, \sigma, \tau)=N \exp \left(\frac{-\log ^{2}\left(1+\tau \frac{\sinh (\tau \sqrt{\log 4})}{\tau \sqrt{\log 4}} \frac{x-\mu}{\sigma}\right)}{2 \tau^{2}}-\frac{\tau^{2}}{2}\right) \text {, }
$$

where $\mu$ is the $x$ value at which the function peaks, $\sigma$ is the Gaussian width, and $\tau$ is the tail parameter. The signal efficiencies for the fourteen considered hadron topologies for the best candidate selection step are $76.5 \%$ and $85 \%$ for the electron and muon modes, respectively. 


\subsection{Background suppression}

In the third selection stage, events from various background sources are suppressed. Backgrounds that peak in the $m_{\mathrm{ES}}$ distribution, and thus mimic the $B \rightarrow X_{s} \ell^{+} \ell^{-}$signal are:

1. Charmonium $B \rightarrow J / \psi X_{s}$ and $B \rightarrow \psi(2 S) X_{s}$ decays, with $J / \psi$ or $\psi(2 S) \rightarrow$ $e^{+} e^{-}$or $\mu^{+} \mu^{-}$, constitute a potentially large background since these decays have a signature identical to the signal and a branching fraction higher by an order of magnitude. They are effectively removed by cutting on the dilepton mass around the $J / \psi$ and $\psi(2 S)$ masses, and provide an excellent control sample for the analysis.

2. $B \rightarrow X_{s} \gamma$ decays followed by a gamma conversion in the material $\gamma \rightarrow e^{+} e^{-}$ are a modest source of peaking background in the electron channel. This background is removed with a minimum cut on the dilepton mass.

3. Hadronic $B \rightarrow D^{(*)} \pi$ decays can also mimic signal decays if a $\pi^{+} \pi^{-}$pair is misidentified as either an $e^{+} e^{-}$or a $\mu^{+} \mu^{-}$pair. The latter is considered in this analysis due to the relatively high $\pi \rightarrow \mu$ misidentification rate.

Purely combinatorial background originates from both $B \bar{B}$ and continuum events. In the case of the $B \bar{B}$ background, the most common source are events in which each $B$ meson decays semileptonically and the final state hadrons combine to form the $B \rightarrow X_{s} \ell^{+} \ell^{-}$candidate.

An initial suppression of the combinatorial backgrounds is achieved by tightening some of the preselection requirements (first 3 cuts listed below). A cut on the maximum value of $m_{X s}$ is particularly useful as backgrounds rise with increasing $m_{X s}$, whereas the signal decreases. Similarly, background events populate $m_{\mathrm{ES}}$ 
evenly, while the signal is concentrated near the $B$ mass. These and additional cuts employed to reduce the backgrounds are:

1. $m_{X s}<1.8 \mathrm{GeV}$.

2. $5.20<m_{\mathrm{ES}}<5.29 \mathrm{GeV}$.

3. $-0.2<\Delta E<0.1 \mathrm{GeV}$.

4. The z-separation between the two lepton at their point of closest approach to the beam spot in the $x y$ plane satisfies $|\Delta z|<0.15 \mathrm{~cm}$.

5. The difference between the energy of the rest of the event and the beam energy in the CM frame $-5.0<\Delta E^{R O E}<2.0 \mathrm{GeV}$, where the rest of the event is formed out of all charged tracks and neutral EMC clusters not included in the $B$ candidate.

6. The beam energy-substituted mass for the rest of the event $m_{E S}^{R O E}>4.9 \mathrm{GeV}$.

7. The lepton pair is required not to contain any electron candidate consistent with a possible gamma conversion.

The above criteria constitute the postselection of the analysis. After the postselection, charmonium background is suppressed by removing candidates with dilepton mass in the following ranges:

$$
\begin{aligned}
& 2.70<m\left(e^{+} e^{-}\right)<3.25 \mathrm{GeV} \\
& 2.80<m\left(\mu^{+} \mu^{-}\right)<3.20 \mathrm{GeV} \\
& 3.45<m\left(e^{+} e^{-}\right)<3.80 \mathrm{GeV} \\
& 3.55<m\left(\mu^{+} \mu^{-}\right)<3.80 \mathrm{GeV}
\end{aligned}
$$



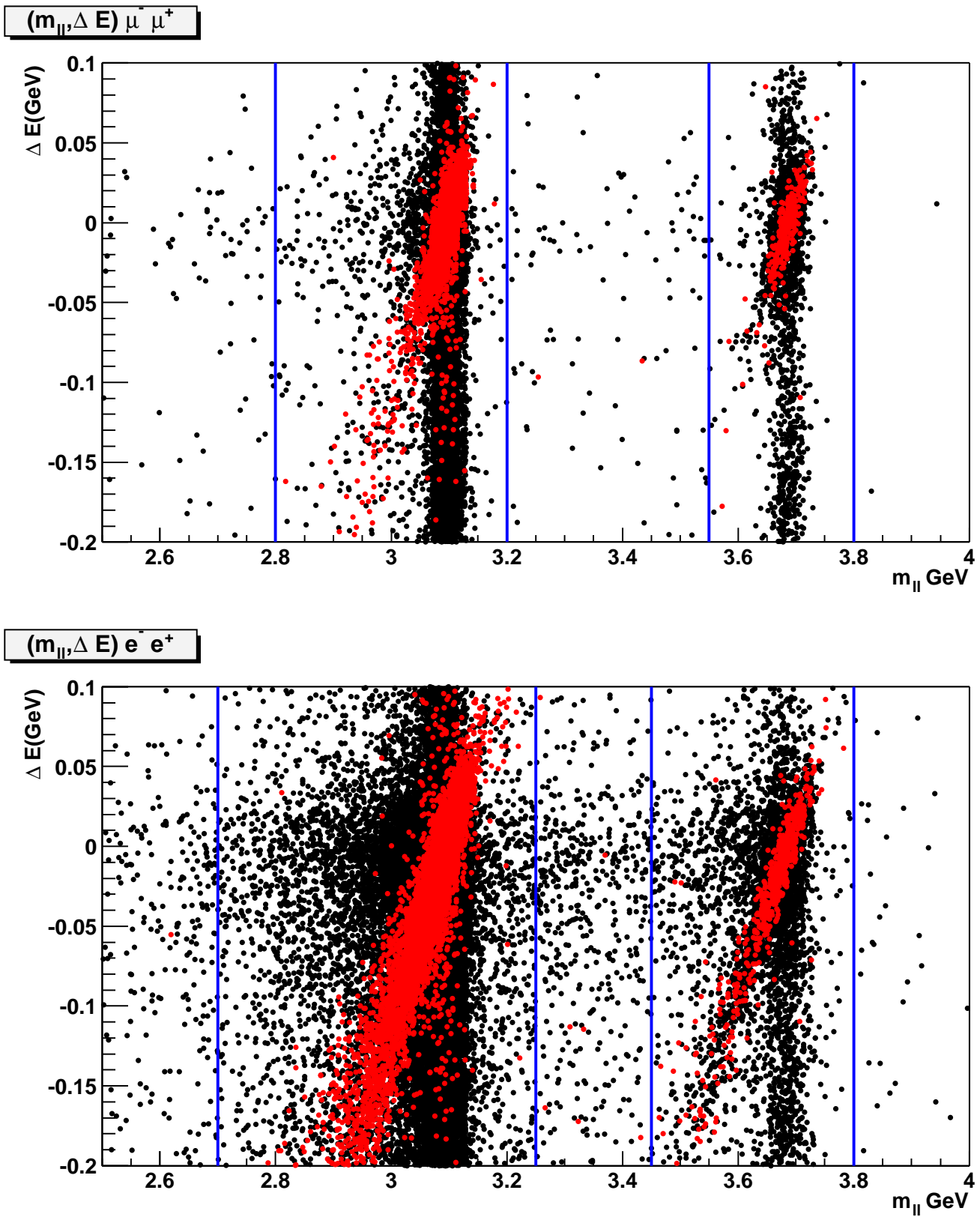

Figure 34: Scatter plots of $\Delta E$ vs. dilepton mass in $B \bar{B} \mathrm{MC}$ events for which the leptons of the reconstructed $B \rightarrow X_{s} \ell^{+} \ell^{-}$candidate are either a $J / \psi$ or $\psi(2 S)$ decay products. The muon channel is shown on top and the electron channel is at the bottom. The vertical bands represent the veto regions. The red points indicate fully reconstructed charmonium $B$ decays, while the black ones correspond to charmonium $B$ decays with miss-reconstructed hadronic part. 
Figure 34 shows scatter plots of $\Delta E$ (for the $B$ decay candidate) as a function of dilepton mass in simulated $B \rightarrow J / \psi X_{s}$ and $B \rightarrow \psi(2 S) X_{s}$ events. These charmonium veto cuts use the Brem-recovered dilepton mass and remove $98.50 \%$ (99.85\%) of this background in the electron (muon) channel. However, we found that a small peaking component still remained in the electron channel and was traced to events in which one or both the electrons erroneously pick up a photon in the Bremsstrahlung recovery process. This may cause a sufficient increase of the dilepton mass to evade the charmonium veto. To reduce the effect of such events, the veto is applied to the dilepton mass before and after the Bremsstrahlung recovery. This additional requirement results in a loss of about $3 \%$ in signal efficiency. Single vs. double charmonium veto comparisons are shown on Fig. 35. Figure 36 illustrates the effect of the charmonium veto on the $m_{\mathrm{ES}}$ distributionfor $B \rightarrow X_{s} \ell^{+} \ell^{-}$ candidates in $B \bar{B}$ MC events for which the leptons of the reconstructed candidate are either a $J / \psi$ or $\psi(2 S)$ decay products.

Another source of peaking background (in the electron channel only) comes from $B \rightarrow X_{s} \gamma$ followed by a conversion of the photon into an $e^{+} e^{-}$pair in the detector material. Figure 37 shows $m_{\mathrm{ES}}$ and dilepton mass distributions for data and $\mathrm{MC}$ in the sideband region $m_{\mathrm{ES}}<5.27 \mathrm{GeV}$. The distributions are provided before and after the likelihood ratio cut (to be described later). A common feature is the excess of data in the very low dilepton mass region, where gamma conversions are expected to contribute. Because of this discrepancy between data and MC in the low $m_{l l}$ region and because this region is not of theoretical interest, we require the dilepton mass to be greater than $0.2 \mathrm{GeV}$. If the already chosen pair fails this requirement the event is rejected. 

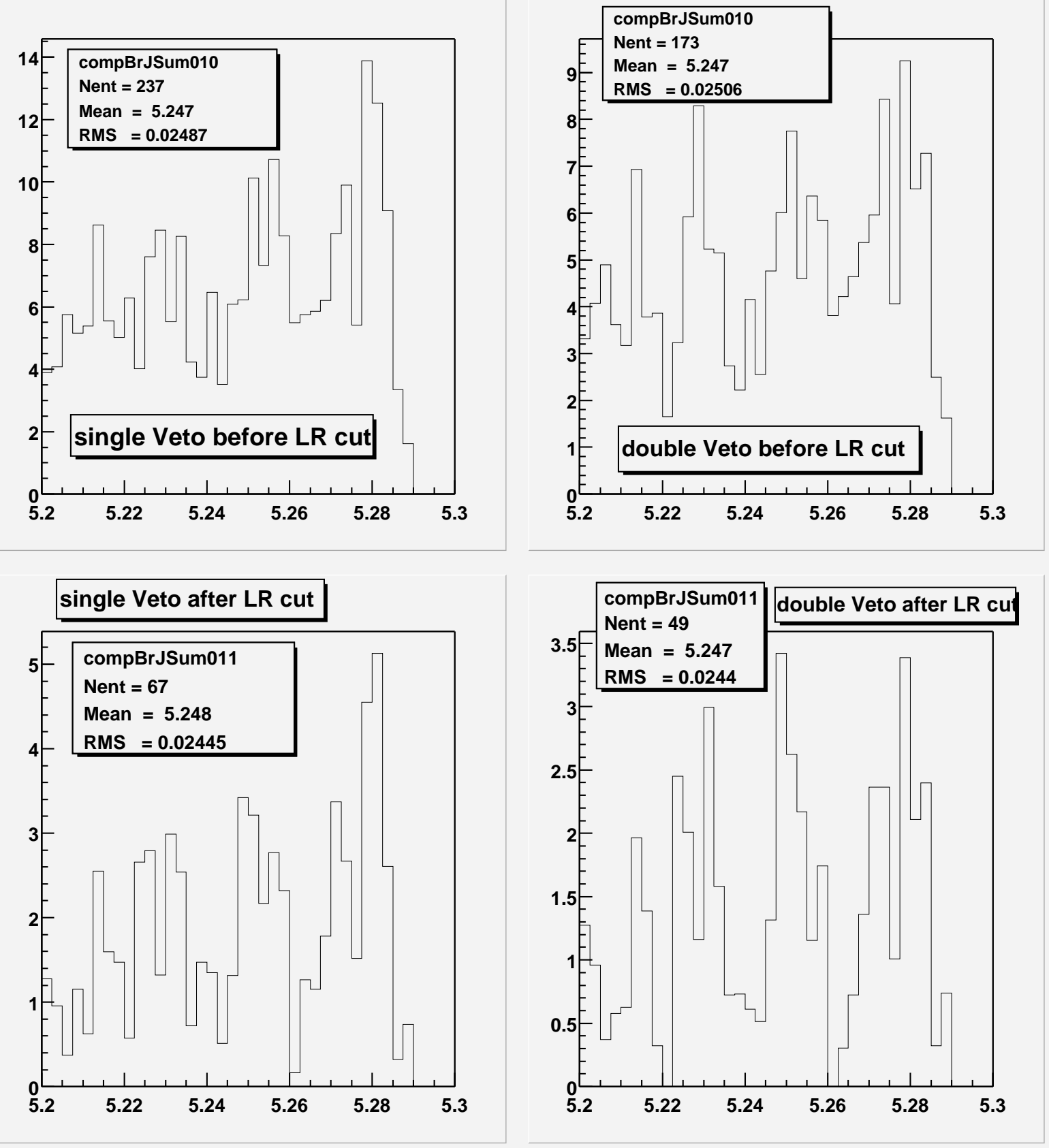

Figure 35: Distributions of $m_{E S}$ for $B \rightarrow X_{s} e^{+} e^{-}$candidates in $B \bar{B}$ MC events for which the dilepton originates from either a $J / \psi$ or $\psi(2 S)$ decay, before likelihood ratio cut (top) and after (bottom). The left-hand plots correspond to applying a single charmonium veto and those on the right correspond to applying a double veto. 

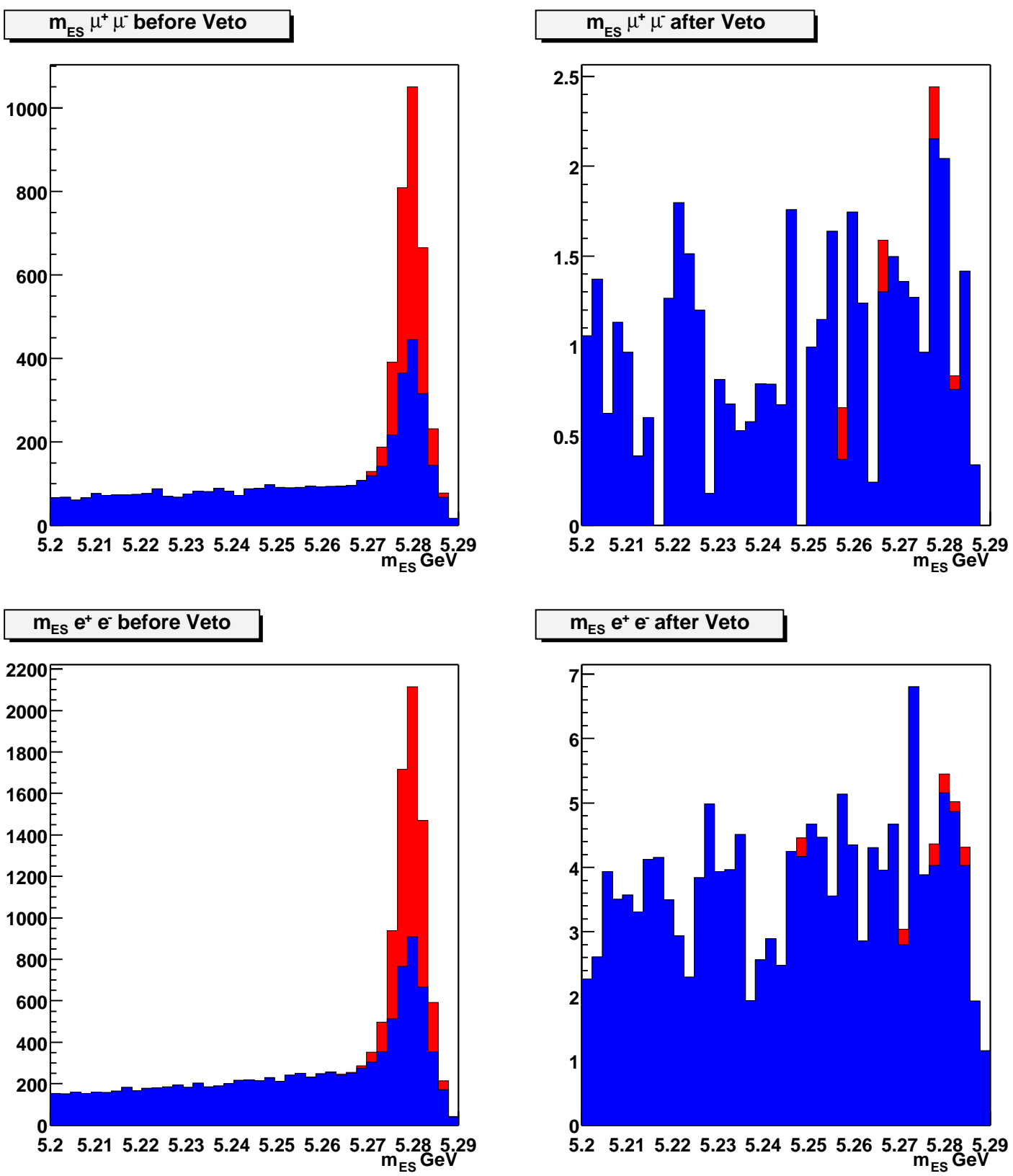

Figure 36: Distributions of $m_{\mathrm{ES}}$ for $B \rightarrow X_{s} \ell^{+} \ell^{-}$candidates in $B \bar{B} \mathrm{MC}$ events for which the leptons of the reconstructed candidate are either a $J / \psi$ or $\psi(2 S)$ decay products. The muon channel is shown on top and the electron channel is at the bottom. All cuts prior to the charmonium veto are applied on the left and the charmonium veto is included on the right. The red area represents fully reconstructed charmonium $B$ decays and the blue corresponds to charmonium $B$ decays with miss-reconstructed hadronic part. 

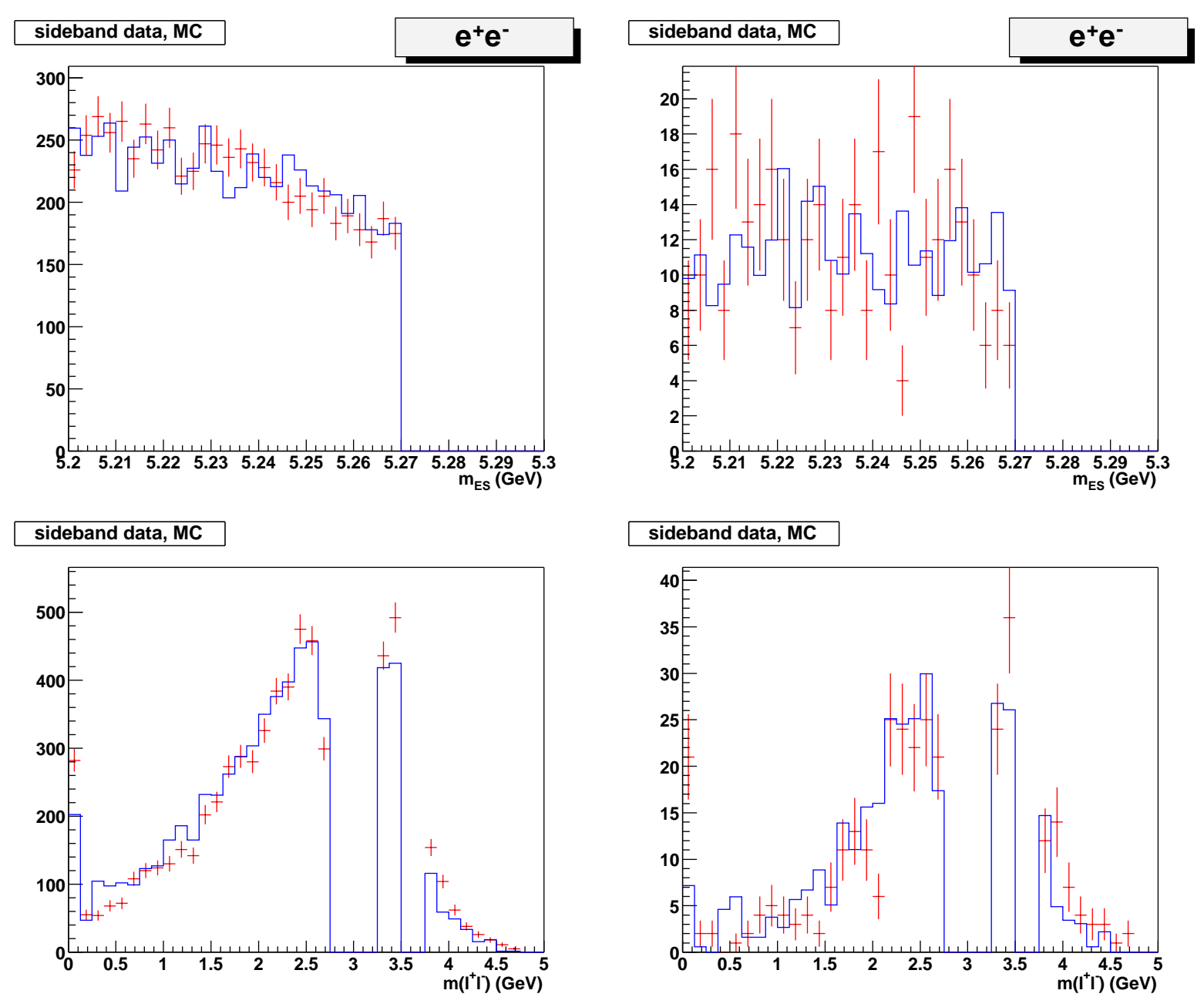

Figure 37: Distributions of $m_{E S}$ (top) and dilepton mass (bottom) for $B \rightarrow$ $X_{s} e^{+} e^{-}$candidates in $m_{\mathrm{ES}}<5.27 \mathrm{GeV}$ sideband on-resonance data (points) and MC (histograms). The plots to the right include the likelihood ratio cut. MC distributions have been normalized to correspond to $81.9 \mathrm{fb}^{-1}$. 
Further suppression of the combinatorial background is achieved by cutting on a likelihood ratio defined as

$$
\mathcal{L}_{R}=\frac{\mathcal{L}^{\text {signal }}}{\mathcal{L}^{\text {signal }}+\mathcal{L}^{B \bar{B}}+\mathcal{L}^{\text {cont }}} .
$$

The likelihood functions for each sample are products of nine PDFs $-P_{i}-$ for that sample, $\mathcal{L}^{\text {sample }}=\prod_{i} P_{i}^{\text {sample }}$, each PDF providing some background separation. The variables we use for the PDFs are:

- Kinematical

$-\Delta E$

- $\triangle E^{R O E}$

$-\mathrm{m}_{\mathrm{ES}}^{\mathrm{ROE}}$

- $\cos \theta_{\text {miss }}$, where $\theta_{\text {miss }}$ is the angle between the missing momentum in the event and the $z$ axis in the CM frame

- Topological

$-\Delta z$

$-\log \left(P_{B v t x}\right)$

- $\left|\cos \theta_{T}\right|$, where $\theta_{T}$ is the angle between the thrust axes of the $B$ candidate and the rest of the event in the CM frame

- $R_{2}$, using only charged tracks

- Angular momentum

$-\cos \theta_{B}$ 
Table 5: Functional forms used to fit the nine components of the $\mathcal{L}_{R}$ likelihood.

\begin{tabular}{ll}
\hline \hline Variable & PDF form \\
\hline$\Delta E$ & Sum of 2 Gaussians \\
$\Delta E^{R O E}$ & Novosibirsk + Gaussian \\
$\mathrm{m}_{\mathrm{ES}}^{\mathrm{ROE}}$ & Novosibirsk + Gaussian \\
$\Delta z$ & Sum of 2 Gaussians \\
$\log \left(P_{B v t x}\right)$ & First order polynomial + exponential \\
$\cos \theta_{\text {miss }}$ & Second order polynomial + sum of 2 exponentials \\
$\cos \theta_{B}$ & Second order polynomial \\
$\left|\cos \theta_{T}\right|$ & First order polynomial + exponential \\
$R_{2}$ & Novosibirsk \\
\hline
\end{tabular}

The variables $\Delta E, \log \left(P_{B v t x}\right)$ and $\cos \theta_{B}$ are the same as those already used for picking the best candidate. The rest of the event variables $-\Delta E^{R O E}$ and $\mathrm{m}_{\mathrm{ES}}^{\mathrm{ROE}}-$ are useful against combinatorial $B \bar{B}$ background, since these events are characterized by significant amount of missing energy due to neutrinos from both $B$ decays. The topological variables rely either on the fact that the charged tracks in a signal event must be consistent with originating from a point $-\Delta z$ and $\log \left(P_{B v t x}\right)-$ or on the overall event shape $-\left|\cos \theta_{T}\right|$ and $R_{2}$ - the latter being useful primarily against continuum. Plots of the nine variables, showing signal and background components along with the fits used to parametrize them as PDFs are shown in Figs. 78 to 94. Table 5 lists the functional form of each PDF. The $\Delta E, \Delta E^{R O E}$ and $\mathrm{m}_{\mathrm{ES}}^{\mathrm{ROE}}$ variables are particularly efficient at rejecting $B \bar{B}$ background, especially for events with two semileptonic decays which are characterized by larger missing energy than signal events. For continuum suppression, the event shape variables $\left|\cos \theta_{T}\right|$ and $R_{2}$ are most useful. 


\subsection{Likelihood ratio cut optimization}

The optimal value of the likelihood ratio cut is determined by maximizing the statistical significance of the signal yield $N_{s i g}$. To this end the cut value is varied, and the corresponding signal yield is extracted from a binned maximum likelihood fit to the $m_{\mathrm{ES}}$ distribution in the MC, see for example the bottom left plot in Fig. 38. The signal significance is defined as $N_{s i g} / \sigma_{s i g}$, where $\sigma_{s i g}$ is the error on $N_{s i g}$ as determined from the fit. The fit function is a sum of an Argus-shape function [24] for the background events and a Gaussian for the truth-matched signal events. The shape parameters of these two functions are determined from separate fits to the background-only and signal-only $m_{\mathrm{ES}}$ distributions, see Fig. 38. The small cross-feed component is included in the background for this optimization method.

As mentioned earlier the background increases rapidly with the increase of multiplicity (or hadronic mass), while at the same time the signal decreases. This brings a dramatic drop of the signal-to-background ratio at high hadronic masses and necessitates a separate likelihood ratio cut optimization in different hadronic mass regions. The optimizations were performed separately for electron and muon modes in three $m_{X s}$ ranges following the method described above. For the $m_{X s}$ ranges we chose: $m_{X s}<0.6 \mathrm{GeV}, 0.6<m_{X s}<1.1 \mathrm{GeV}$ and $m_{X s}>1.1 \mathrm{GeV}$, the values roughly correspond to the boundaries between the three MC signal components. The likelihood ratio distributions in these three regions are shown in Figs. 39 through 44 .

Given the small expected signal yield and the substantial background for candidates with final state topologies containing 3 pions, when optimizing we also considered a case where all 3-pion modes are excluded. In addition we explored the effect of defining the likelihood ratio in such a way as to account for the difference 
sigBMesN
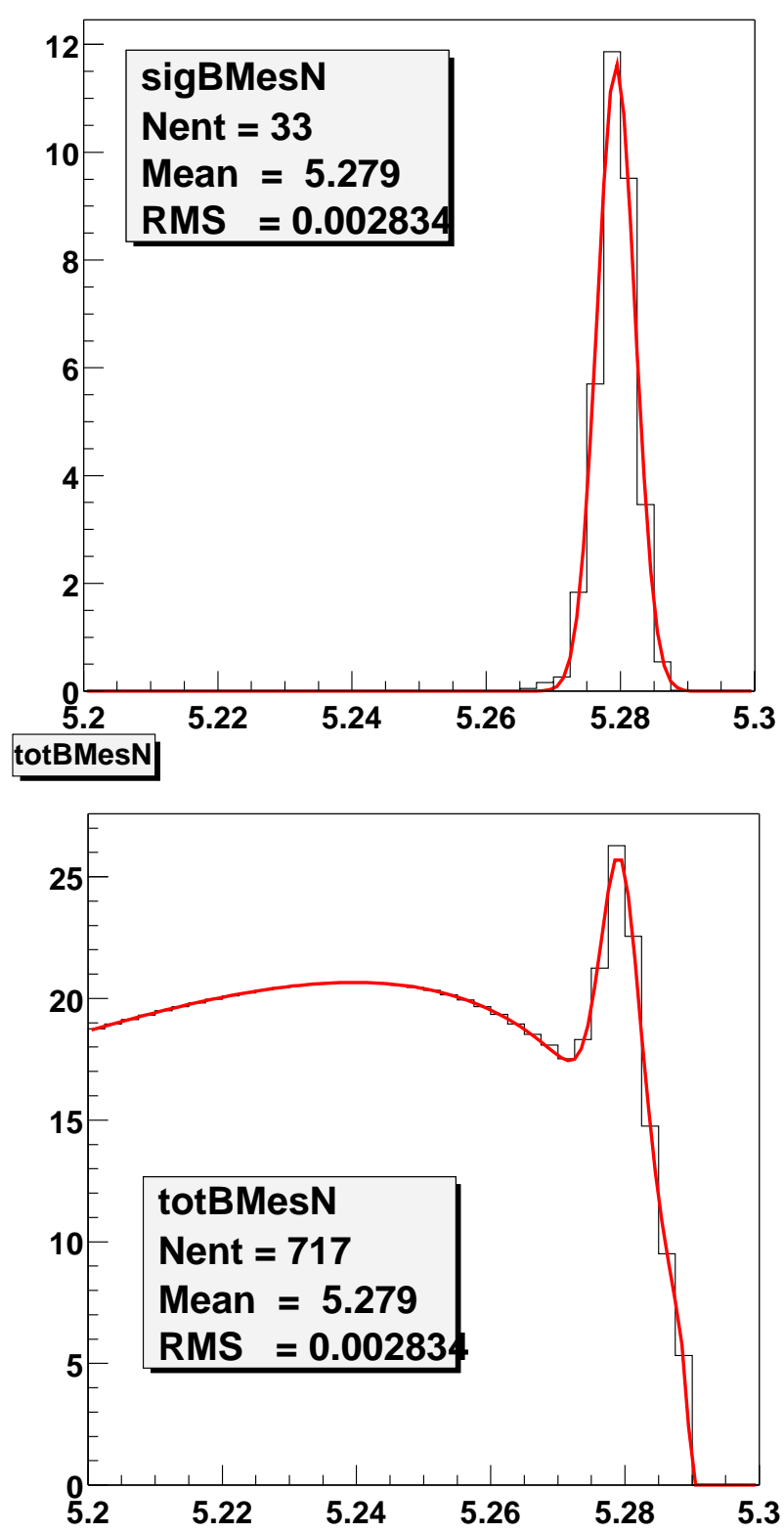

bkgBMesN

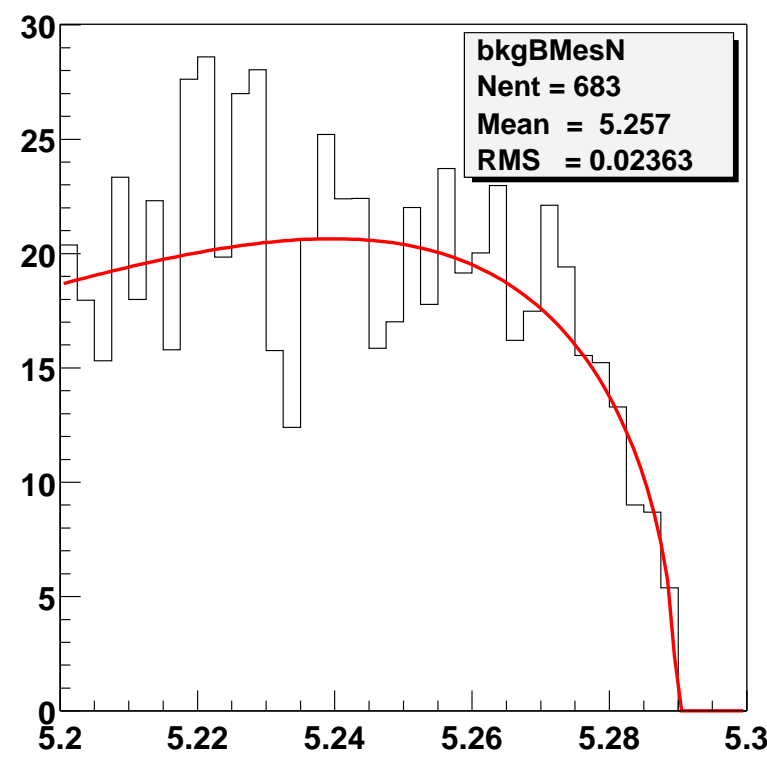

Figure 38: Distribution of $m_{E S}$ in the electron channel with a particular set of likelihood ratio cuts for the following components in the MC: truthmatched $B \rightarrow X_{s} e^{+} e^{-}$signal (top left), total background including cross-feed (top right), and sum of signal and smoothed background (bottom left). The lines represent the result of a binned maximum likelihood fit to each distribution separately. Entries have been normalized to correspond to $81.9 \mathrm{fb}^{-1}$. 

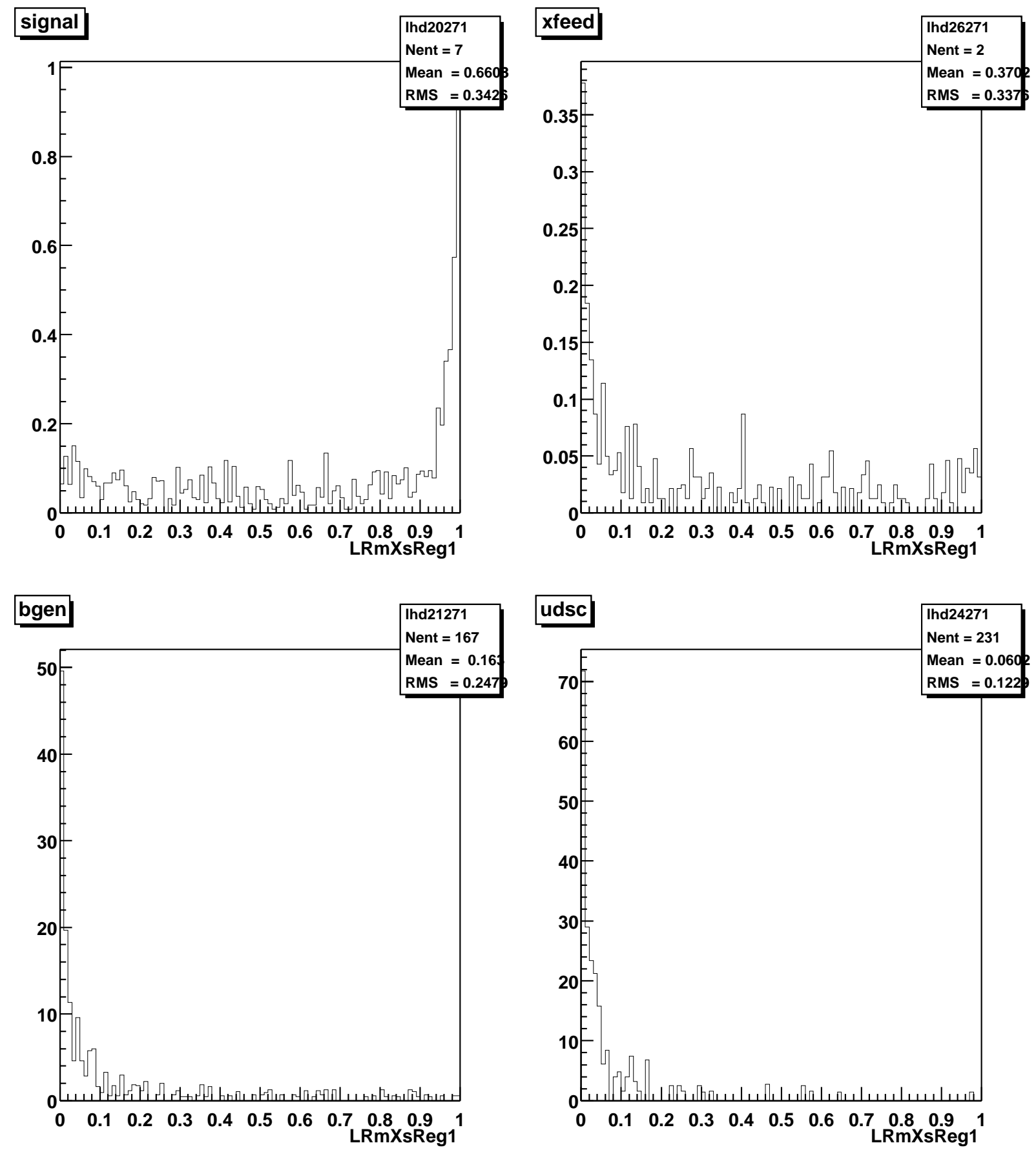

Figure 39: Distributions of the likelihood ratio for $B \rightarrow X_{s} e^{+} e^{-}$candidates with $M_{X_{s}}<0.6 \mathrm{GeV}$ in truth-matched signal (top left), cross-feed (top right), $B \bar{B}$ (bottom left) and continuum (bottom right) events. 

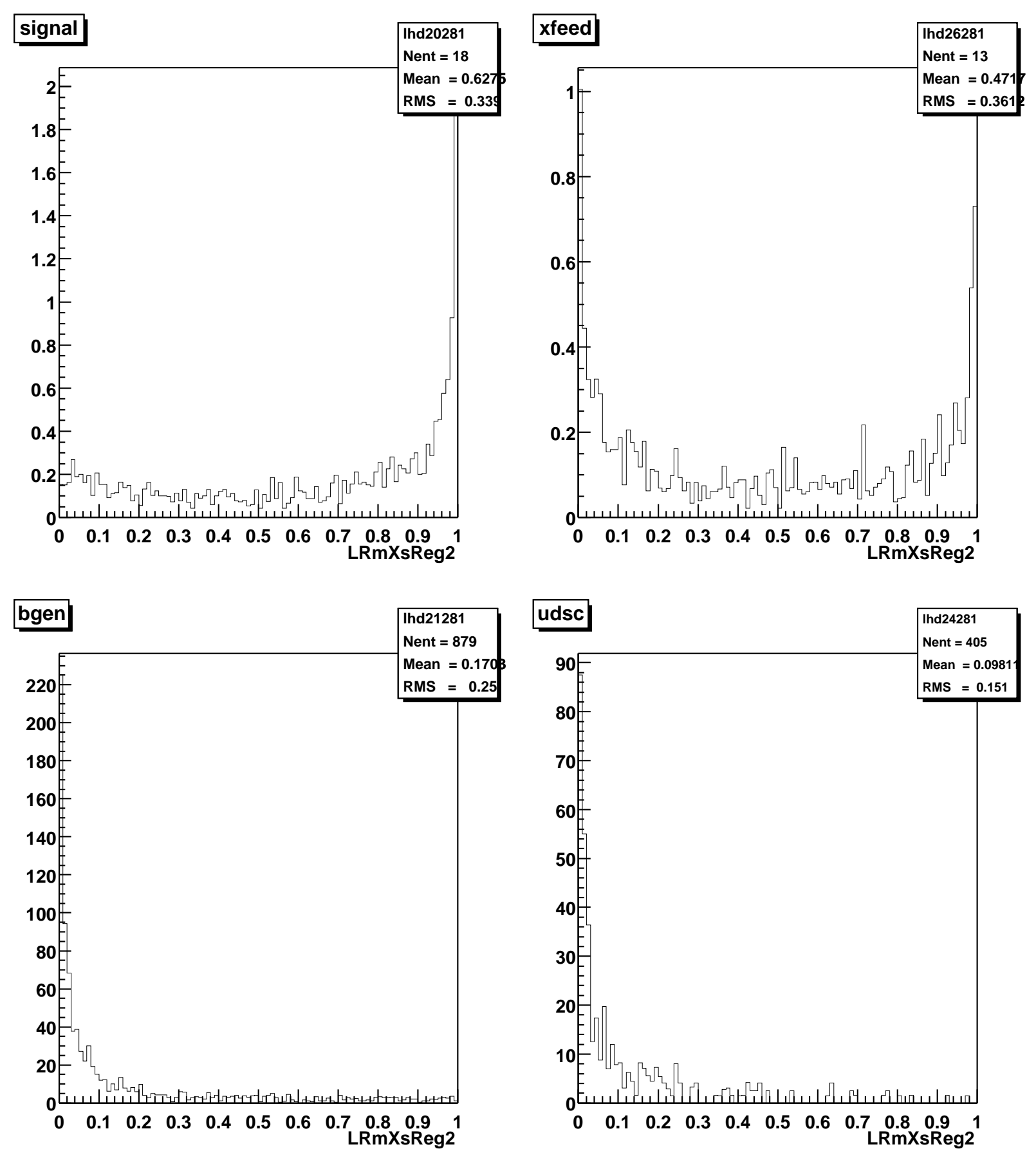

Figure 40: Distributions of the likelihood ratio for $B \rightarrow X_{s} e^{+} e^{-}$candidates with $0.6<M_{X_{s}}<1.1 \mathrm{GeV}$ in truth-matched signal (top left), crossfeed (top right), $B \bar{B}$ (bottom left) and continuum (bottom right) events. 

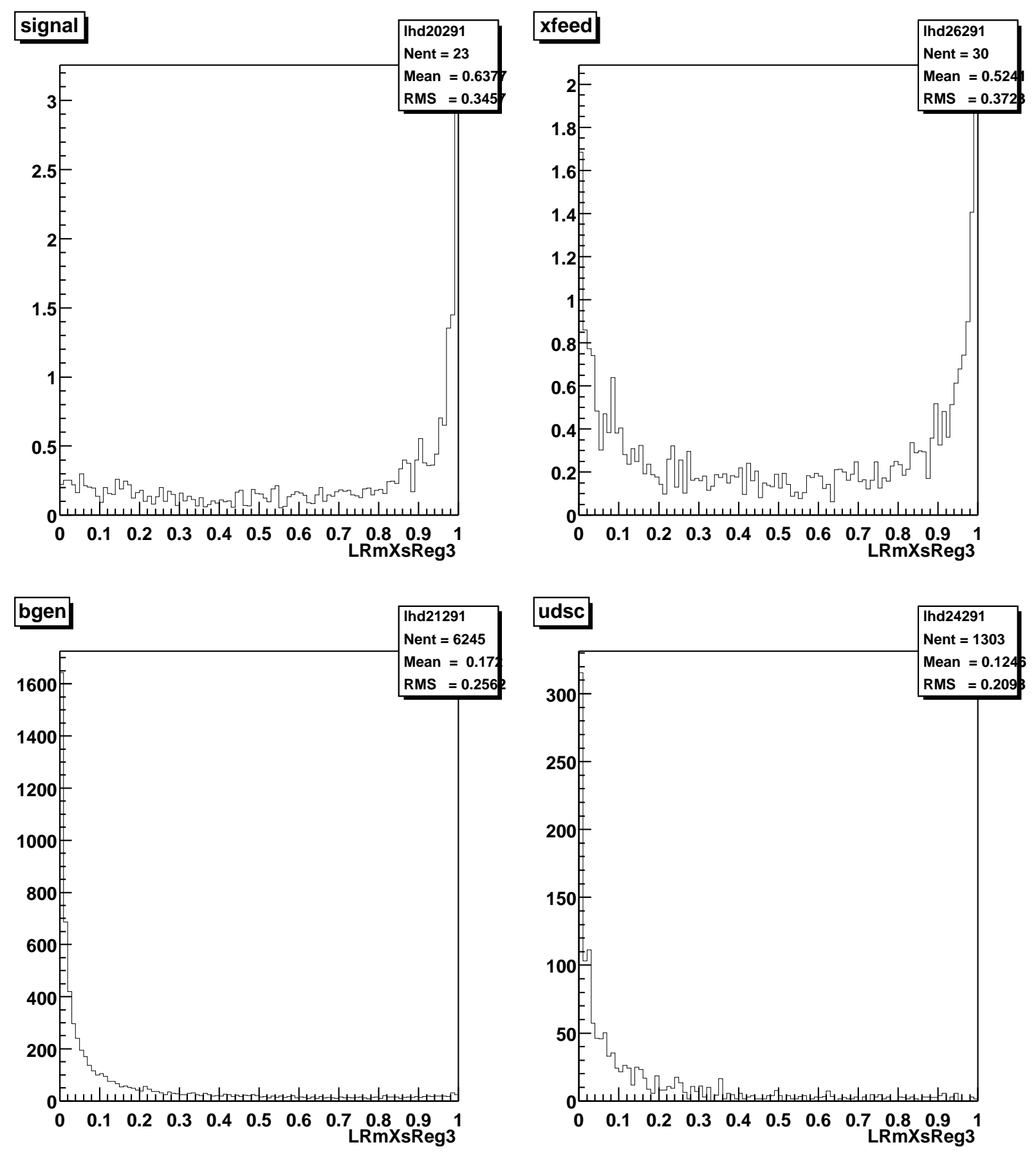

Figure 41: Distributions of the likelihood ratio for $B \rightarrow X_{s} e^{+} e^{-}$candidates with $1.1<M_{X_{s}}<1.8 \mathrm{GeV}$ in truth-matched signal (top left), crossfeed (top right), $B \bar{B}$ (bottom left) and continuum (bottom right) events. 

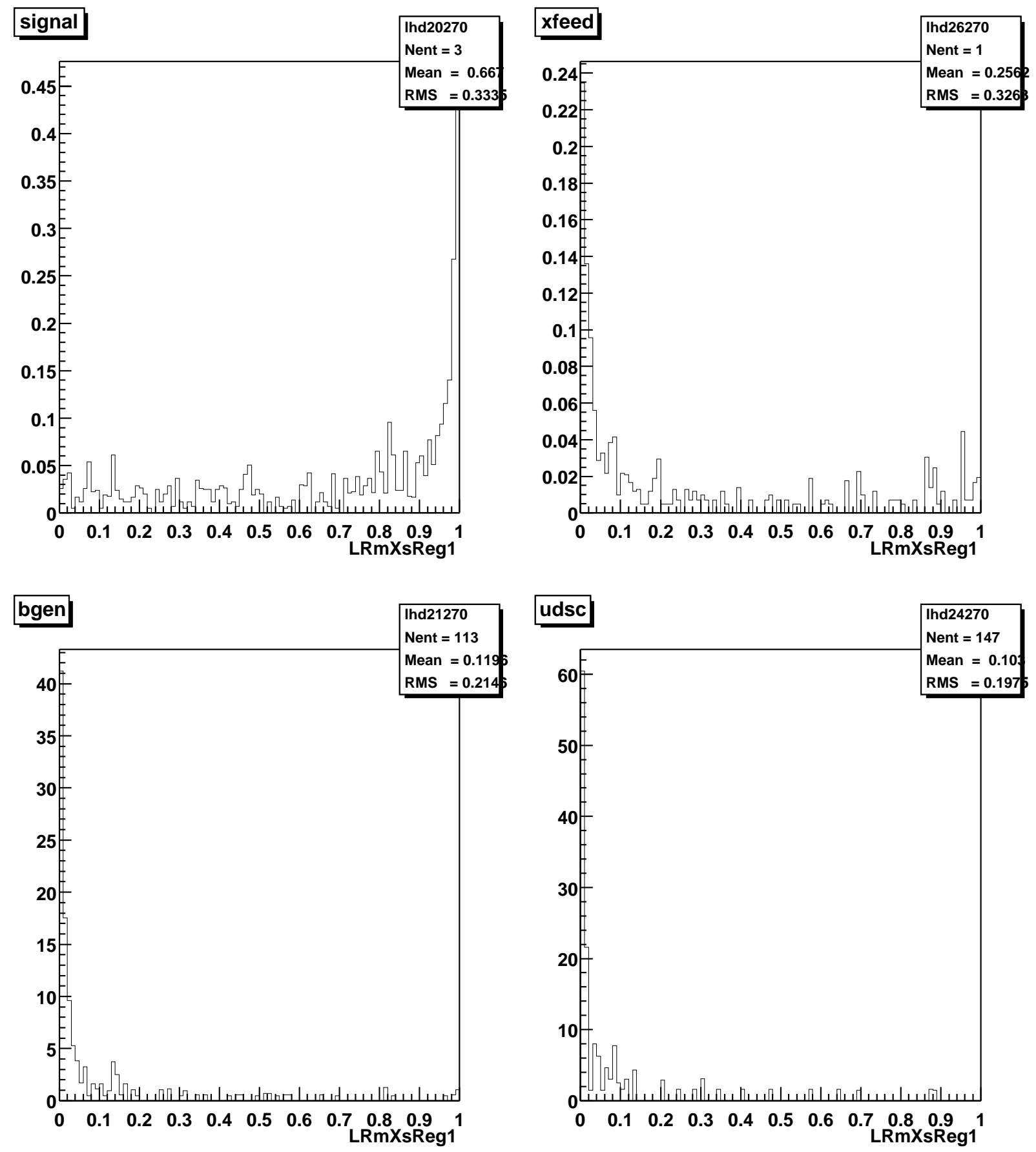

Figure 42: Distributions of the likelihood ratio for $B \rightarrow X_{s} \mu^{+} \mu^{-}$candidates with $M_{X_{s}}<0.6 \mathrm{GeV}$ in truth-matched signal (top left), cross-feed (top right), $B \bar{B}$ (bottom left) and continuum (bottom right) events. 

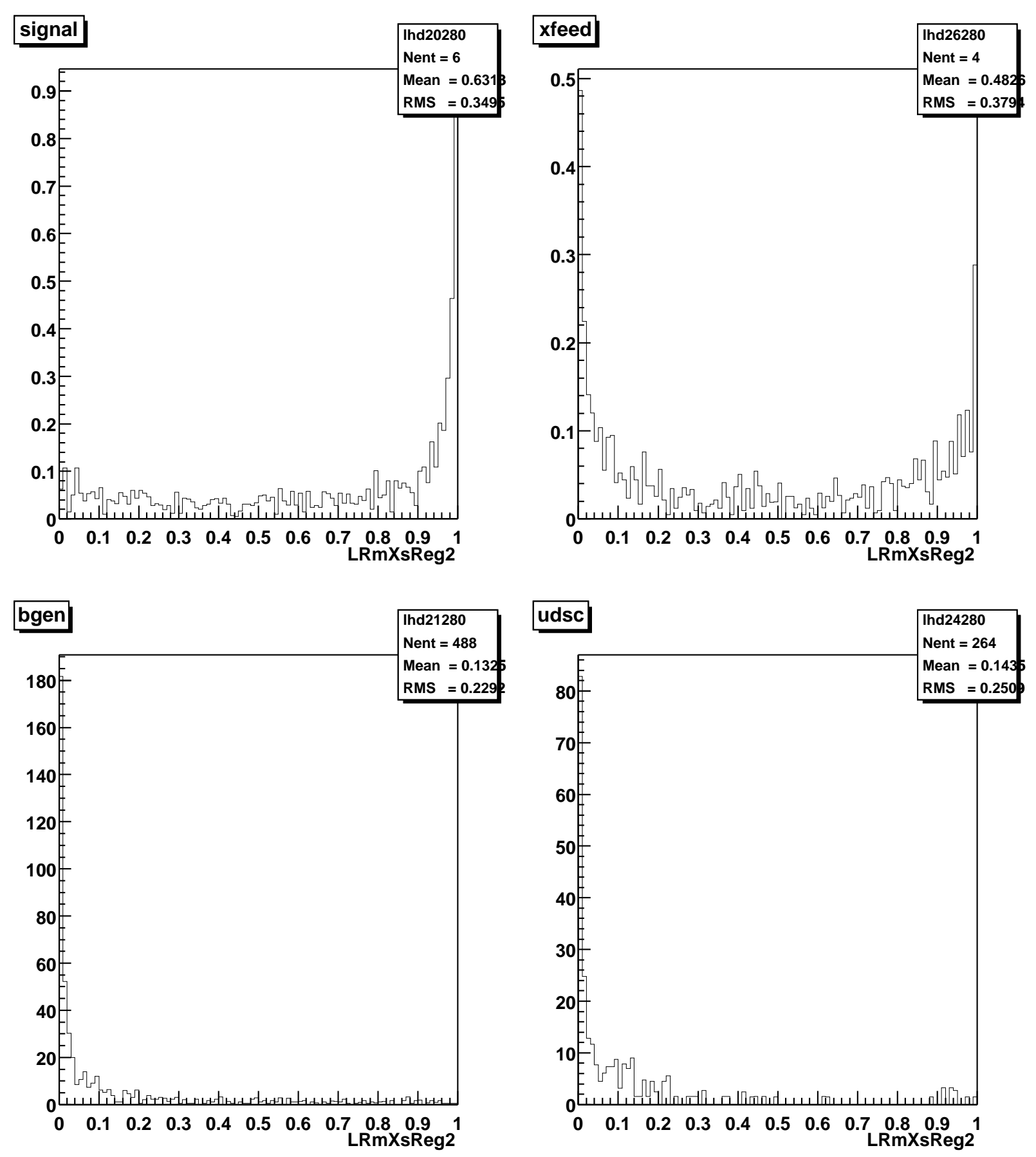

Figure 43: Distributions of the likelihood ratio for $B \rightarrow X_{s} \mu^{+} \mu^{-}$candidates with $0.6<M_{X_{s}}<1.1 \mathrm{GeV}$ in truth-matched signal (top left), crossfeed (top right), $B \bar{B}$ (bottom left) and continuum (bottom right) events. 

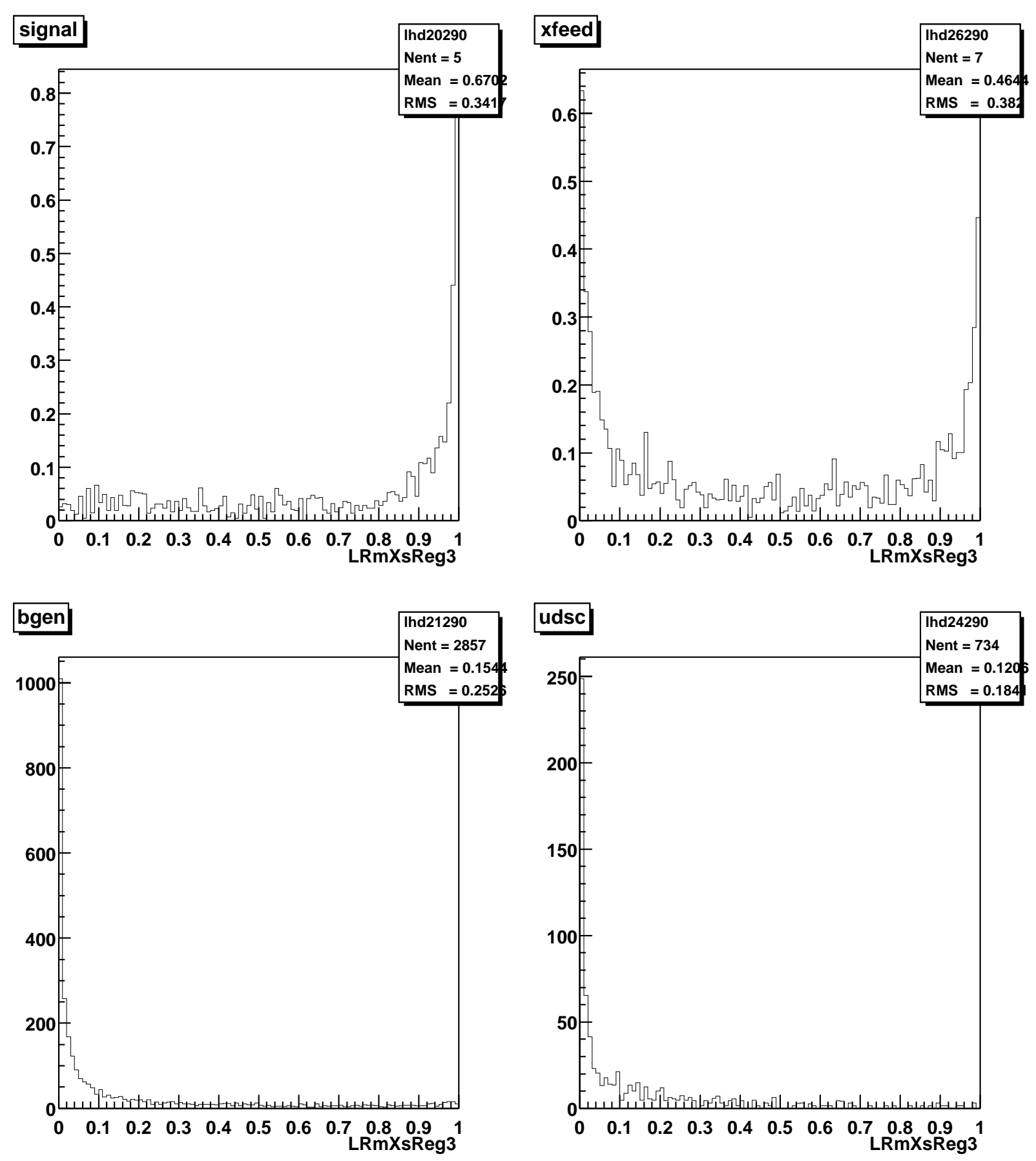

Figure 44: Distributions of the likelihood ratio for $B \rightarrow X_{s} \mu^{+} \mu^{-}$candidates with $1.1<M_{X_{s}}<1.8 \mathrm{GeV}$ in truth-matched signal (top left), crossfeed (top right), $B \bar{B}$ (bottom left) and continuum (bottom right) events. 
in the number of candidates from $B \bar{B}$ and continuum events

$$
\mathcal{L}_{R}^{\prime}=\frac{\mathcal{L}^{\text {signal }}}{\mathcal{L}^{\text {signal }}+f_{B \bar{B}} \mathcal{L}^{B \bar{B}}+f_{\text {cont }} \mathcal{L}^{\text {cont }}},
$$

where $f_{B \bar{B}}$ and $f_{\text {cont }}$ represent the fraction of the background due to $B \bar{B}$ and continuum events: $f_{B \bar{B}}=79 \%(75 \%)$ and $f_{\text {cont }}=21 \%(25 \%)$ in the electron (muon) channel. The results of the different optimization methods and the optimal cut values for each case are presented in Table 5.1. Based on these results we apply the following set of cuts as the final stage of the event selection:

1. The 3 pion modes are excluded. As a result, the final sample contains a total of 10 different modes. Given the final states produced in our particular MC model (see Table 3) and the fraction of $K \ell^{+} \ell^{-}$modes (see Table 2), this set of 10 modes corresponds to approximately $50 \%$ of all possible final states. If one assumes that the missing $K_{L}$ component is equal to the $K_{S}$ component, this fraction becomes $70 \%$.

2. Likelihood ratio cuts for $B \rightarrow X_{s} e^{+} e^{-}$:

$$
\begin{array}{r}
\mathcal{L}_{R}>0.3 \text { for } m_{X s}<0.6 \mathrm{GeV}, \\
\mathcal{L}_{R}>0.4 \text { for } 0.6<m_{X s}<1.1 \mathrm{GeV}, \\
\mathcal{L}_{R}>0.9 \text { for } m_{X s}>1.1 \mathrm{GeV} .
\end{array}
$$

3. Likelihood ratio cuts for $B \rightarrow X_{s} \mu^{+} \mu^{-}$:

$$
\begin{array}{r}
\mathcal{L}_{R}>0.2 \text { for } m_{X s}<0.6 \mathrm{GeV}, \\
\mathcal{L}_{R}>0.6 \text { for } 0.6<m_{X s}<1.1 \mathrm{GeV}, \\
\mathcal{L}_{R}>0.9 \text { for } m_{X s}>1.1 \mathrm{GeV} .
\end{array}
$$




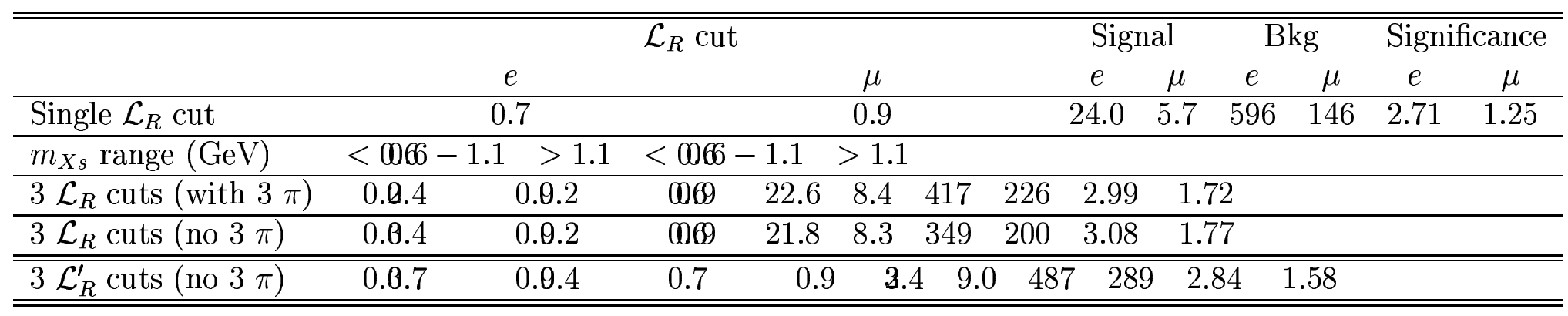

Tabe 5.1: Signal and background yields for different likelihood ratio cut optimization approaches. The first row shows the results obtained with a single cut, the following rows correspond to separate cuts in different $m_{X s}$ regions, with and without the 3-pion modes. The last row corresponds to the likelihood ratio $\mathcal{L}_{R}^{\prime}$ defined in Eq. 5.4. The likelihood ratio cut values are given on the left-hand side in the order of the $m_{X s}$ ranges. 
An improvement of the signal significance by about 0.3 and $0.5 \sigma$ is achieved in the electron and muon channels compared to the single $\mathcal{L}_{R}$ cut case. Figures 45 and 46 show the final $m_{X s}$ spectrum of reconstructed truth-matched MC signal in the electron and muon chanels.

The MC-based signal reconstruction efficiencies at the different stages of the analysis for the 14 final state topologies are presented in Tables 28 and 29. By far, the largest source of inefficiency is due to the requirement that all final state particles are reconstructed and correctly identified (the column "in lists" in the tables). Similarly, Tables 30 and 31 show efficiencies for MC background events in the electron and muon channels. 


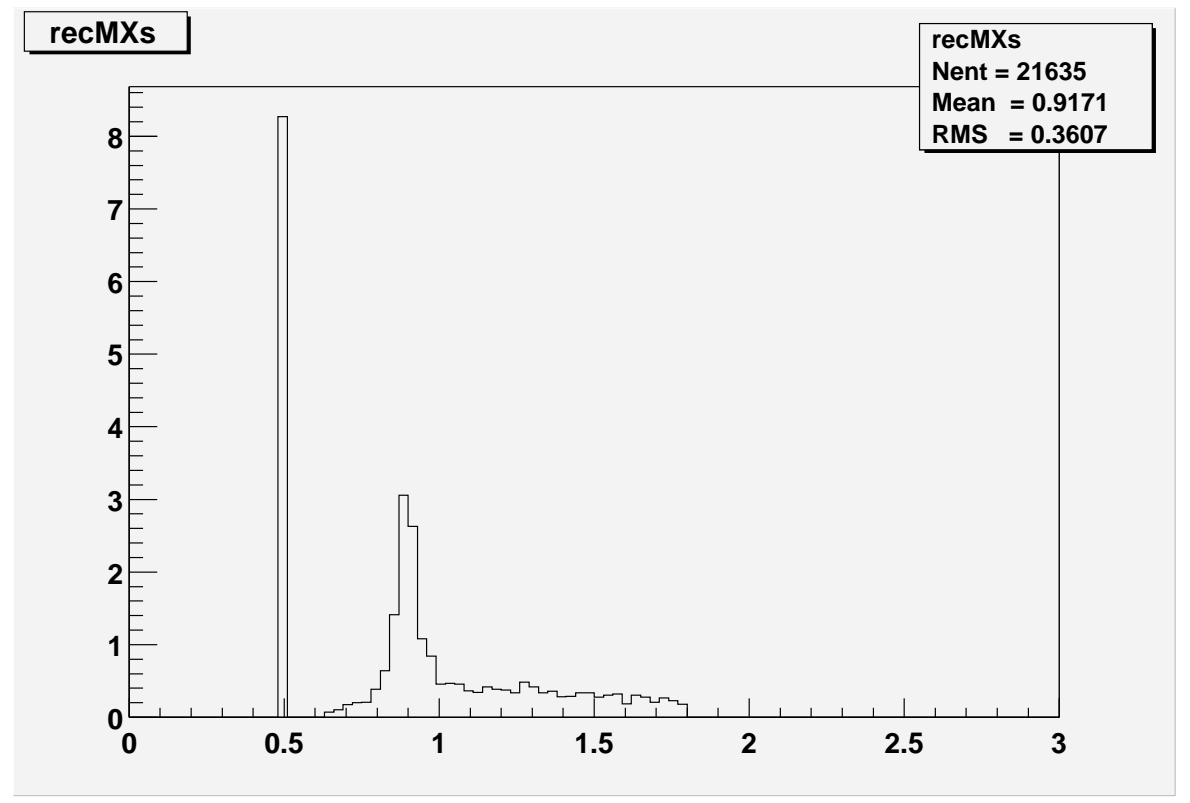

Figure 45: Distributions of hadronic mass for correctly reconstructed $B \rightarrow$ $X_{s} e^{+} e^{-}$signal after the nominal likelihood ratio cuts are applied. The selection also excludes final states containing 3 pions. The Belle model is used for the signal MC.

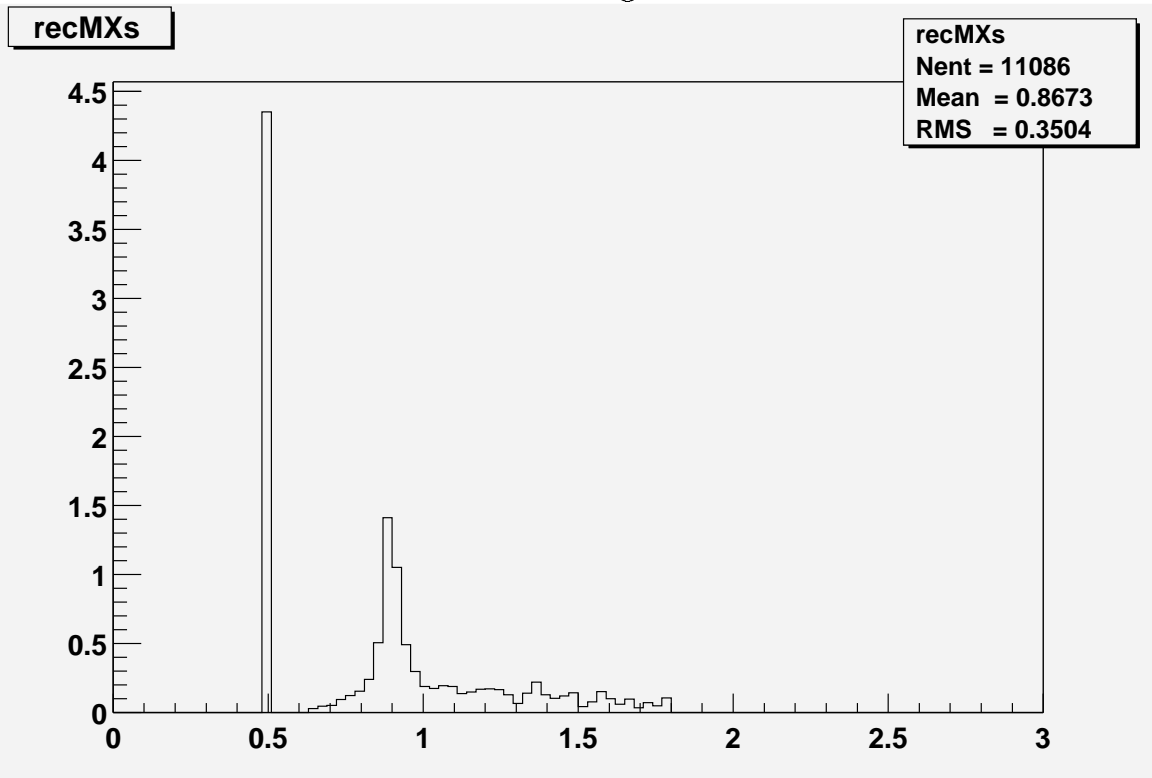

Figure 46: Distributions of hadronic mass for correctly reconstructed $B \rightarrow$ $X_{s} \mu^{+} \mu^{-}$signal after the likelihood ratio cuts are applied. The selection also excludes final states containing 3 pions. The Belle model is used for the signal MC. 


\section{H A P T E R 6}

\section{Cross checks and validation}

In this analysis we use MC generated signal and background events and detector simulation to tune the background suppression cuts and estimate the final signal efficiency. In order to test the validity of such approach we use the following control samples to compare directly the real detector response and cut effectiveness to the ones obtained from simulation:

1. Charmonium sample.

2. $X_{s} e^{ \pm} \mu^{\mp}$ sample.

3. Off-resonance sample.

4. On-resonance sideband sample.

\subsection{Charmonium control sample}

The charmonium decays $B \rightarrow J / \psi X_{s}$ and $B \rightarrow \psi(2 S) X_{s}$ have signatures identical to that of our signal and provide an excellent control sample to compare signal reconstruction efficiencies, albeit in restricted dilepton mass regions. The control sample is obtained at the stage when the charmonium veto is applied, after the 
Table 6: Definition of the topology number of the hadronic system $X_{s}$.

\begin{tabular}{lr}
\hline \hline$X_{s}$ Topology & Id \# \\
\hline$K$ & 1 \\
$K \pi^{0}$ & 2 \\
$K \pi$ & 3 \\
$K \pi \pi^{0}$ & 4 \\
$K \pi \pi$ & 5 \\
\hline$K_{S}^{0}$ & 20 \\
$K_{S}^{0} \pi^{0}$ & 21 \\
$K_{S}^{0} \pi$ & 22 \\
$K_{S}^{0} \pi \pi^{0}$ & 23 \\
$K_{S}^{0} \pi \pi$ & 24 \\
\hline
\end{tabular}

postselection and before the likelihood ratio cuts. The events in the sample are required to satisfy one of the following dilepton mass cuts:

$$
\begin{aligned}
& 2.70<m\left(e^{+} e^{-}\right)<3.25 \mathrm{GeV} \\
& 2.85<m\left(\mu^{+} \mu^{-}\right)<3.15 \mathrm{GeV} \\
& 3.50<m\left(e^{+} e^{-}\right)<3.76 \mathrm{GeV} \\
& 3.60<m\left(\mu^{+} \mu^{-}\right)<3.74 \mathrm{GeV}
\end{aligned}
$$

where the $e^{+} e^{-}$invariant mass is computed after Bremsstrahlung recovery. In order to obtain a sample with higher charmonium purity the above cuts are tighter than the ones used for the veto. First we compare data and MC for this raw sample, which includes B candidates with $m_{\mathrm{ES}}$ values between 5.20 and $5.29 \mathrm{GeV}$ implying that the hadronic system is not correctly reconstructed for a significant fraction of the sample, see Figs. 47 to 49 . Figure 48 shows the $X_{s}$ system topology following the definition in Table 6 .

Second, we compare data and MC for fully reconstructed $B \rightarrow J / \psi X_{s}$ and $B \rightarrow \psi(2 S) X_{s}$ decays by only considering events from the signal region in $m_{\mathrm{ES}}$ : 

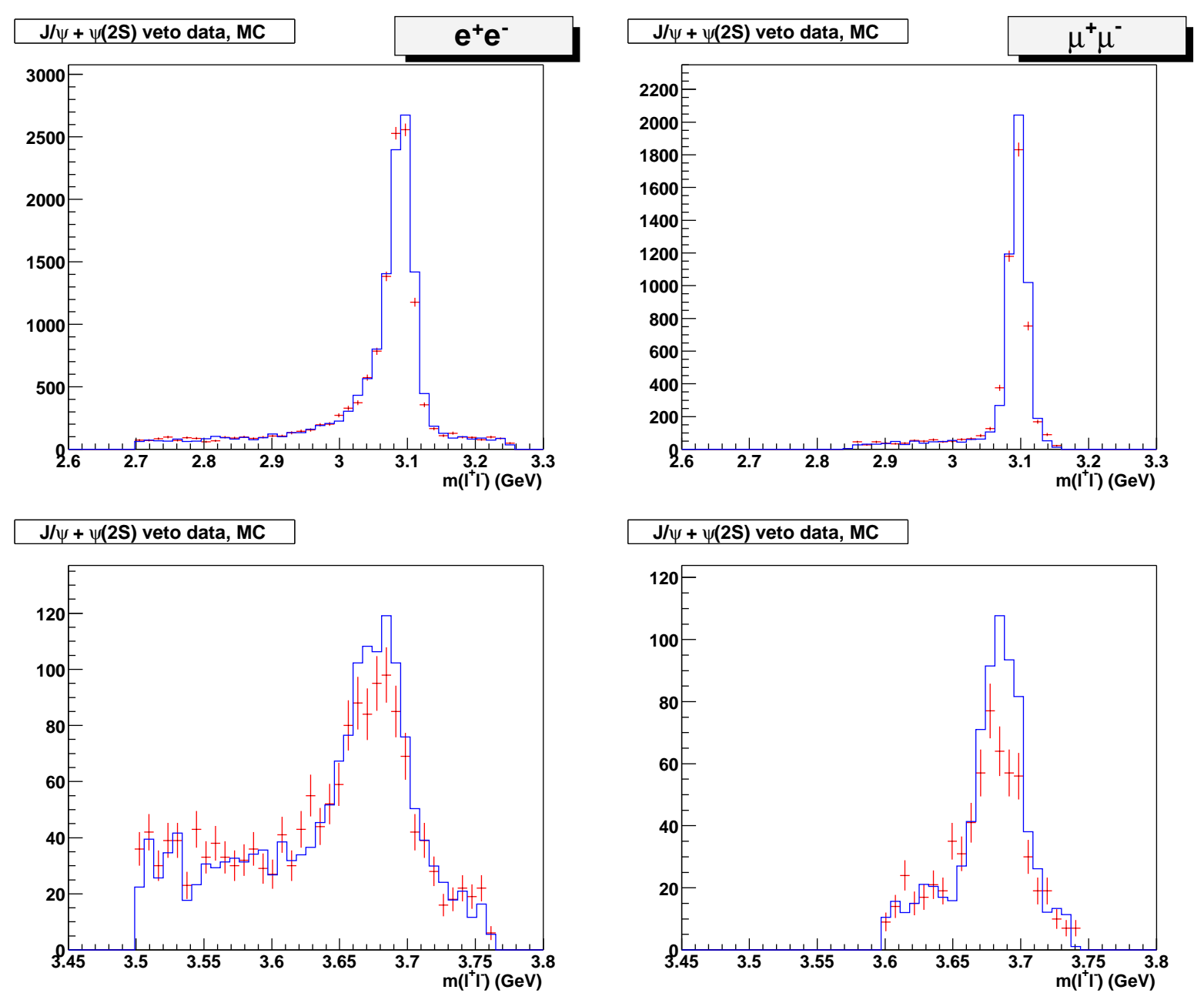

Figure 47: Distributions of dilepton invariant mass for $B \rightarrow X_{s} \ell^{+} \ell^{-}$candidates in the charmonium veto sample for electrons (left) and muons (right). The top and bottom rows are for the $J / \psi$ and $\psi(2 S)$ mass regions, respectively. The points represent the data and the histograms the MC, scaled to correspond to an integrated luminosity of $81.9 \mathrm{fb}^{-1}$. 

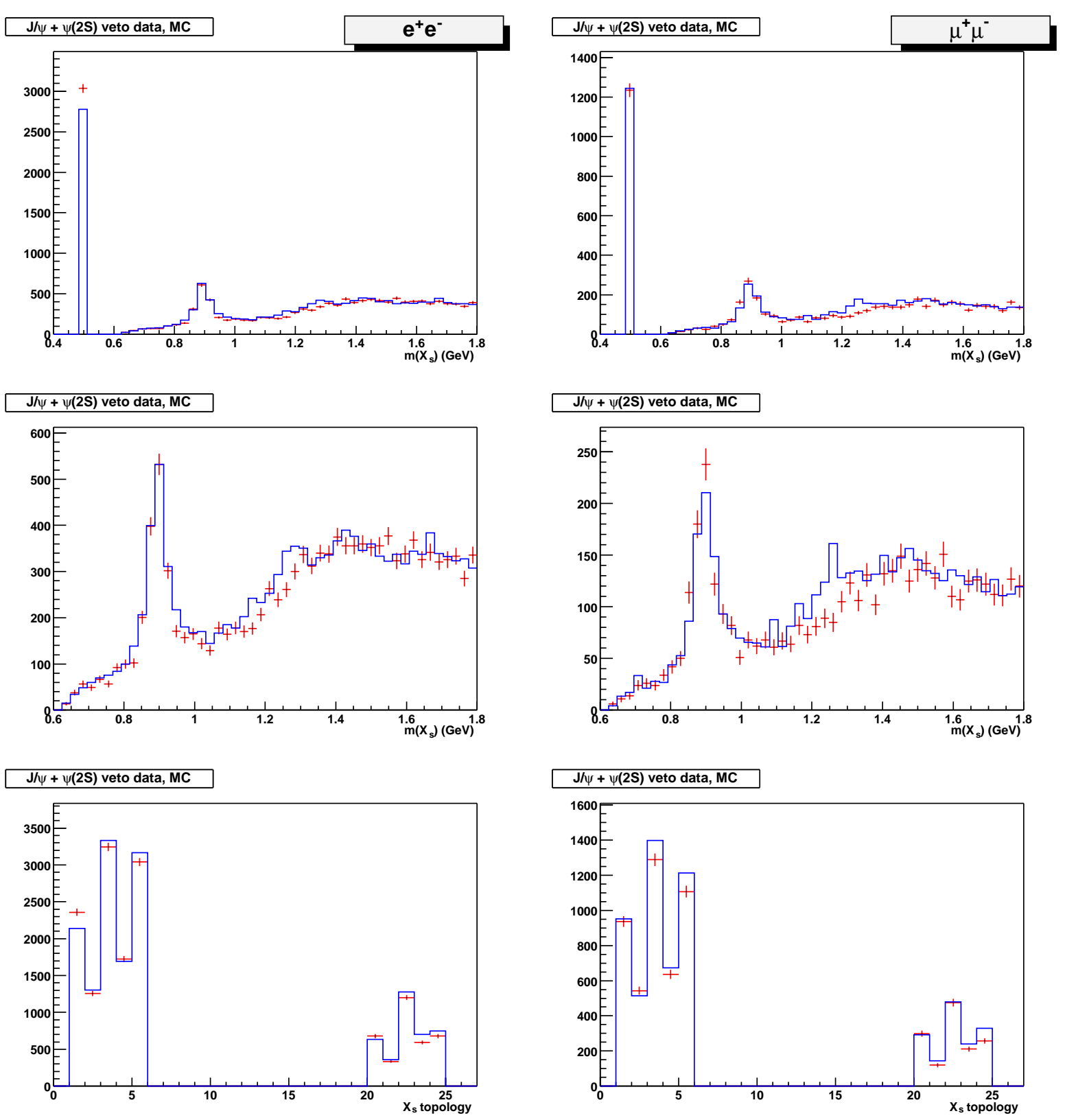

Figure 48: Distributions of hadronic invariant mass and final state topology (see Table 6) for $B \rightarrow X_{s} \ell^{+} \ell^{-}$candidates in the charmonium veto sample for electrons (left) and muons (right). The points represent the data and the histograms the MC, scaled to correspond to an integrated luminosity of $81.9 \mathrm{fb}^{-1}$. 

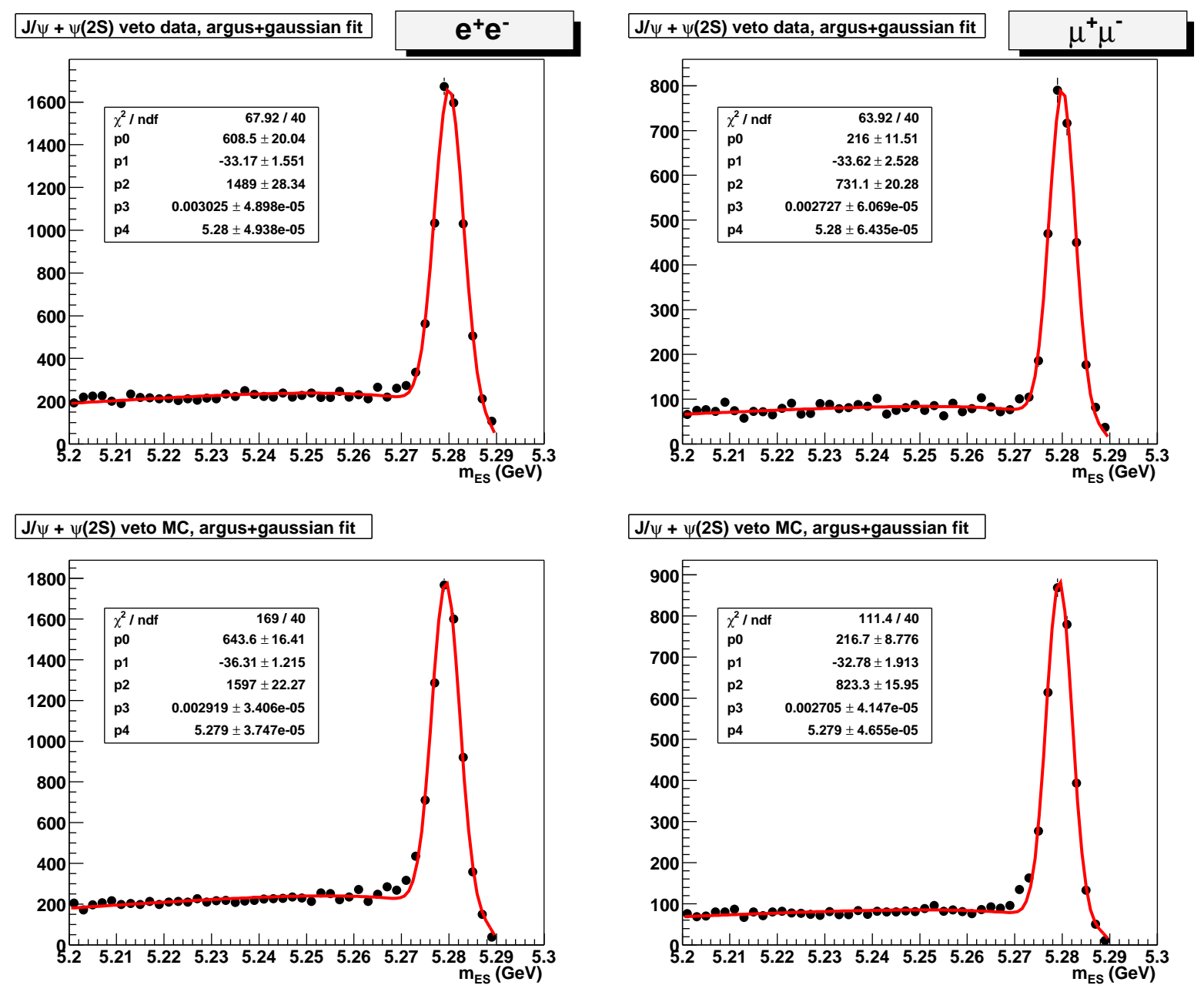

Figure 49: Distributions of $m_{E S}$ for $B \rightarrow X_{s} \ell^{+} \ell^{-}$candidates in the charmonium veto sample for electrons (left) and muons (right). The top row corresponds to data and the bottom shows the MC, scaled to correspond to an integrated luminosity of $81.9 \mathrm{fb}^{-1}$. 
$5.27<m_{\mathrm{ES}}<5.29 \mathrm{GeV}$. We estimate and subtract the combinatorial background in the signal region by using a scale factor obtained from a $\chi^{2}$ fit to the $m_{\mathrm{ES}}$ distributions, see Fig. 49. The maximum likelihood fit uses a Gaussian to describe the signal component and an Argus function for the combinatorial background. The end point of the Argus function is fixed to $5.29 \mathrm{GeV}$, all other fit parameters are allowed to float. Distributions obtained after background subtraction approximate the distributions to be expected from correctly reconstructed charmonium $B$ decays. Figures 6.1 and 6.2 , in particular, show comparisons for the nine variables entering the background-suppression likelihood ratio. Other distributions are shown in Figs. 50-51. A slight excess is observed in the data for the $\mathrm{Ke}^{+} e^{-}$final state and a slight deficit in the $K_{1}(1270)^{0} \ell^{+} \ell^{-}$mode, see below.

Third, we apply the nominal values of the likelihood ratio cut. The resulting distributions are shown in Figs. 52-56. Of particular interest is the ratio between the absolute yields in the data and the MC. This ratio is shown in Tables 7 and 8 for each reconstructed $B \rightarrow J / \psi X$ topology in the electron and muon channels. Tables 9 and 10 show the same results for $B \rightarrow \psi(2 S) X$ candidates. This comparison is important since it tests whether the simulation reliably estimates the efficiency to reconstruct $B$ decays into final states with a signature identical to that of the $B \rightarrow X_{s} \ell^{+} \ell^{-}$signal. However, the current knowledge of inclusive $B \rightarrow J / \psi X$ and $B \rightarrow \psi(2 S) X$ decays is limited: $\mathcal{B}(B \rightarrow J / \psi X)=(1.15 \pm 0.06) \%$ and $\mathcal{B}(B \rightarrow \psi(2 S) X)=(0.35 \pm 0.05) \%$ [2], i.e. the total branching fractions are known with a relative uncertainty of $5.2 \%$ and $14 \%$, respectively. Furthermore, only a few exclusive decay channels have been measured with a level of accuracy close to this. The branching fractions for decays into $J / \psi K$ and $J / \psi K^{*}$ are known to $5-6 \%$ and $7-9 \%$, respectively (the uncertainties vary between $B^{0}$ and $B^{+}$decays). Higher mass final states are rather unknown. This means that the comparison be- 

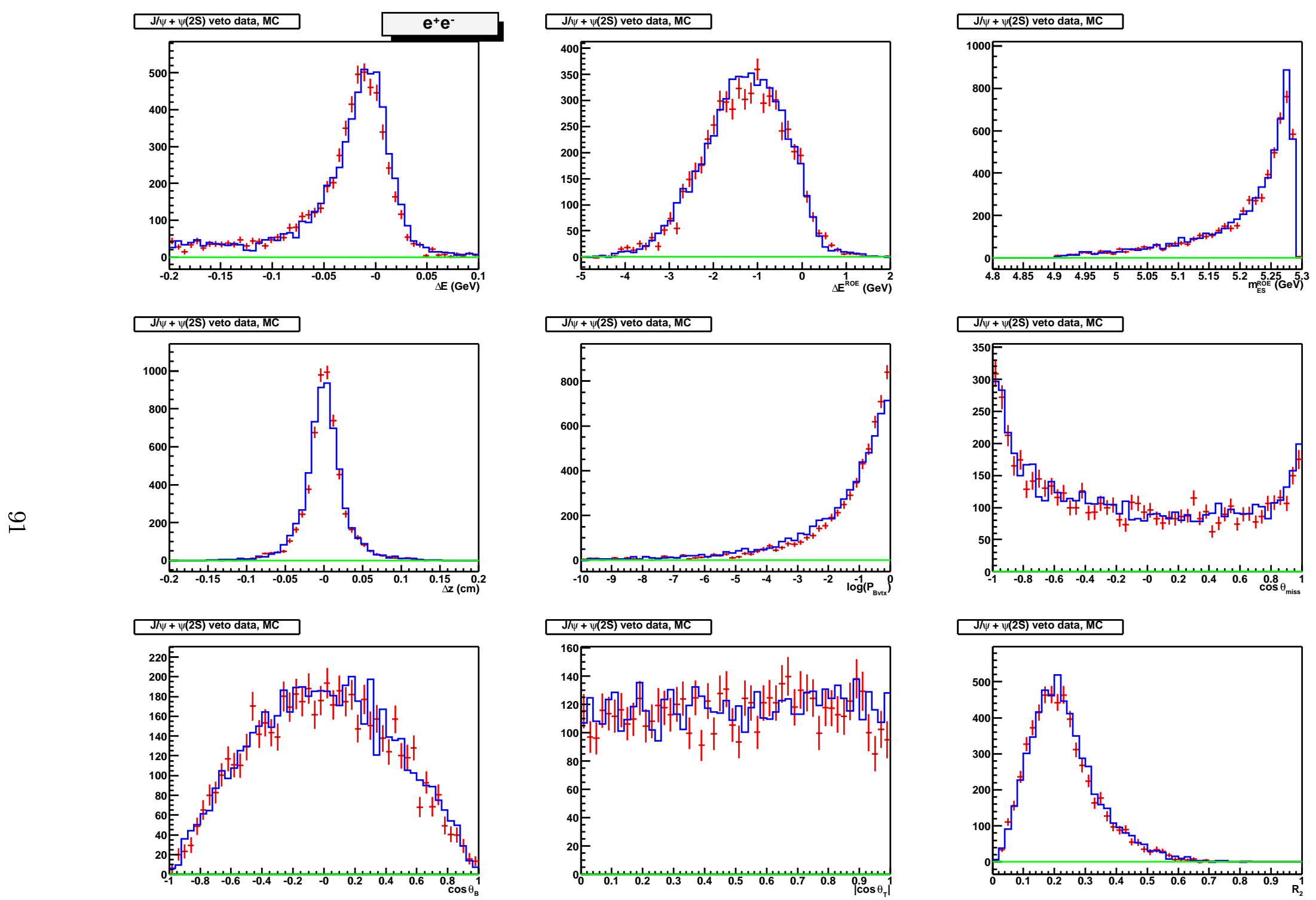

Figure 6.1: Distributions of the background-suppression variabes entering the likelihood ratio for the backgroundsubtracted charmonium events in the electron channel. The points represent the data and the histograms the MC, scaled to correspond to an integrated luminosity of $81.9 \mathrm{fb}^{-1}$. 

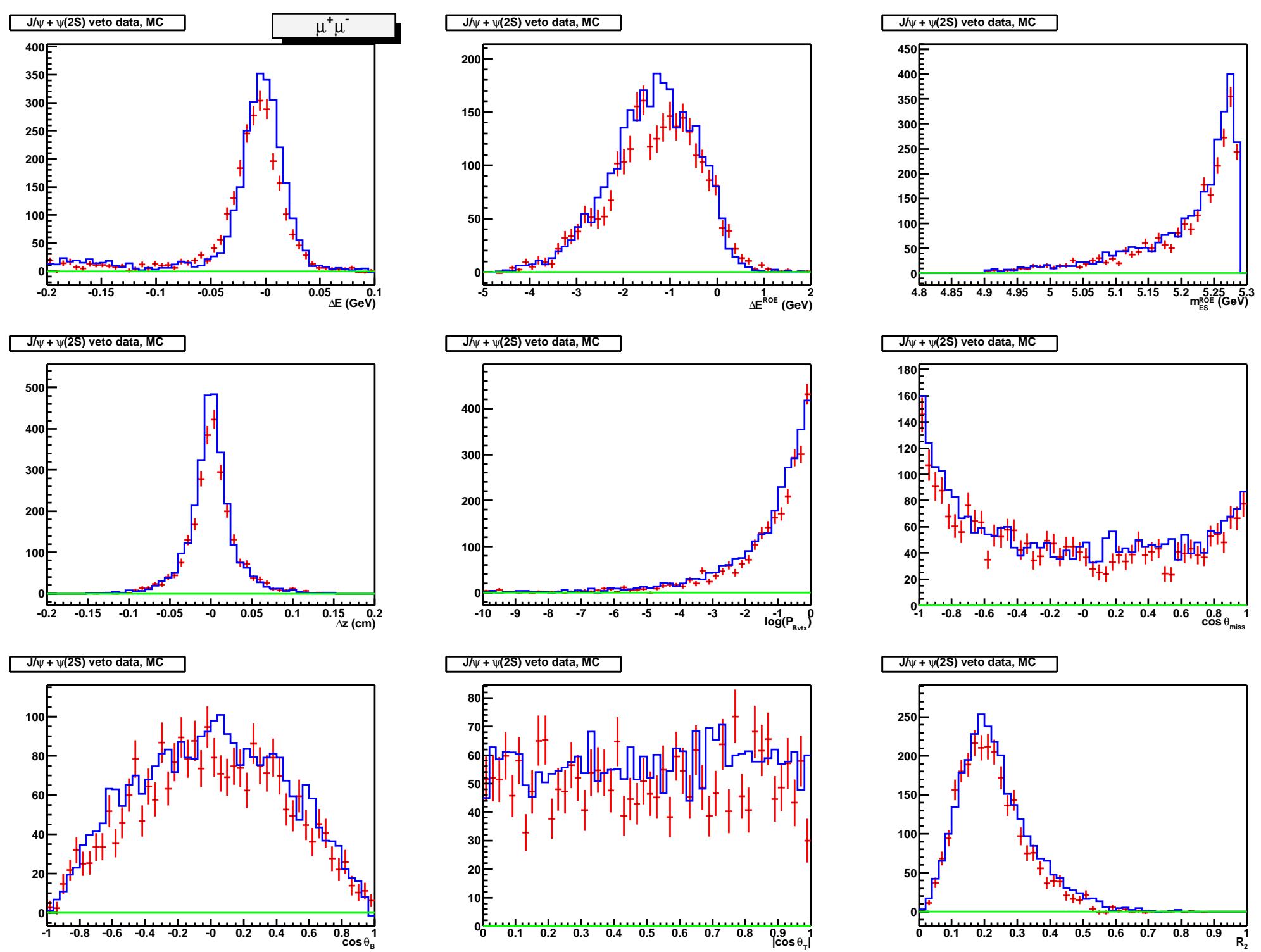

Figure 6.2: Distributions of the background-suppression variabes entering the likelihood ratio for the backgroundsubtracted charmonium events in the muon channel. The points represent the data and the histograms the $\mathrm{MC}$, scaled to correspond to an integrateduminosity of $81.9 \mathrm{fb}^{-1}$. 

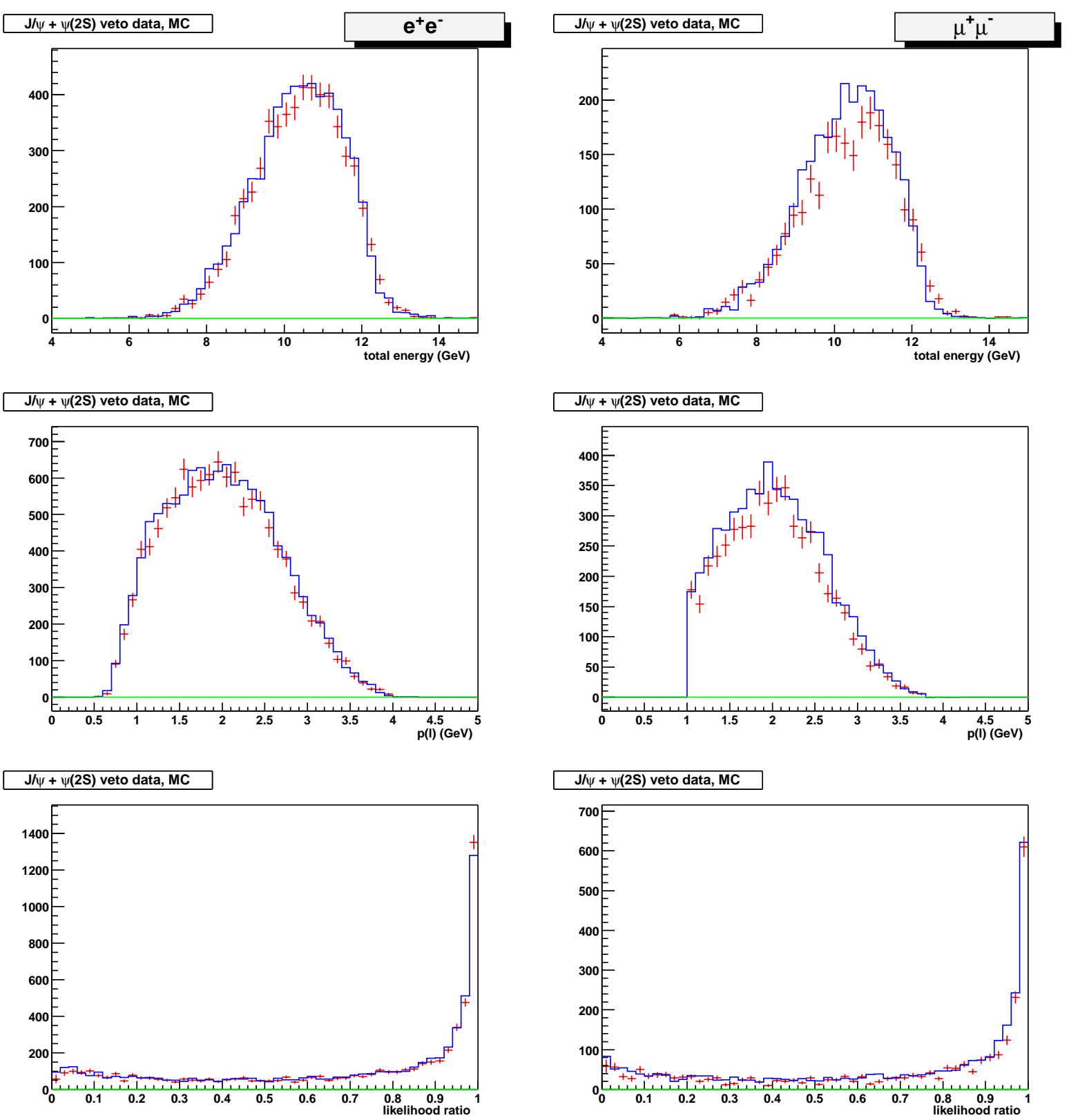

Figure 50: Distributions of total event energy, lepton momentum (both in the lab frame), and likelihood ratio for $B \rightarrow X_{s} \ell^{+} \ell^{-}$candidates in the charmonium veto sample for electrons (left) and muons (right) after background subtraction. The points represent the data and the histograms the MC, scaled to correspond to an integrated luminosity of $81.9 \mathrm{fb}^{-1}$. 

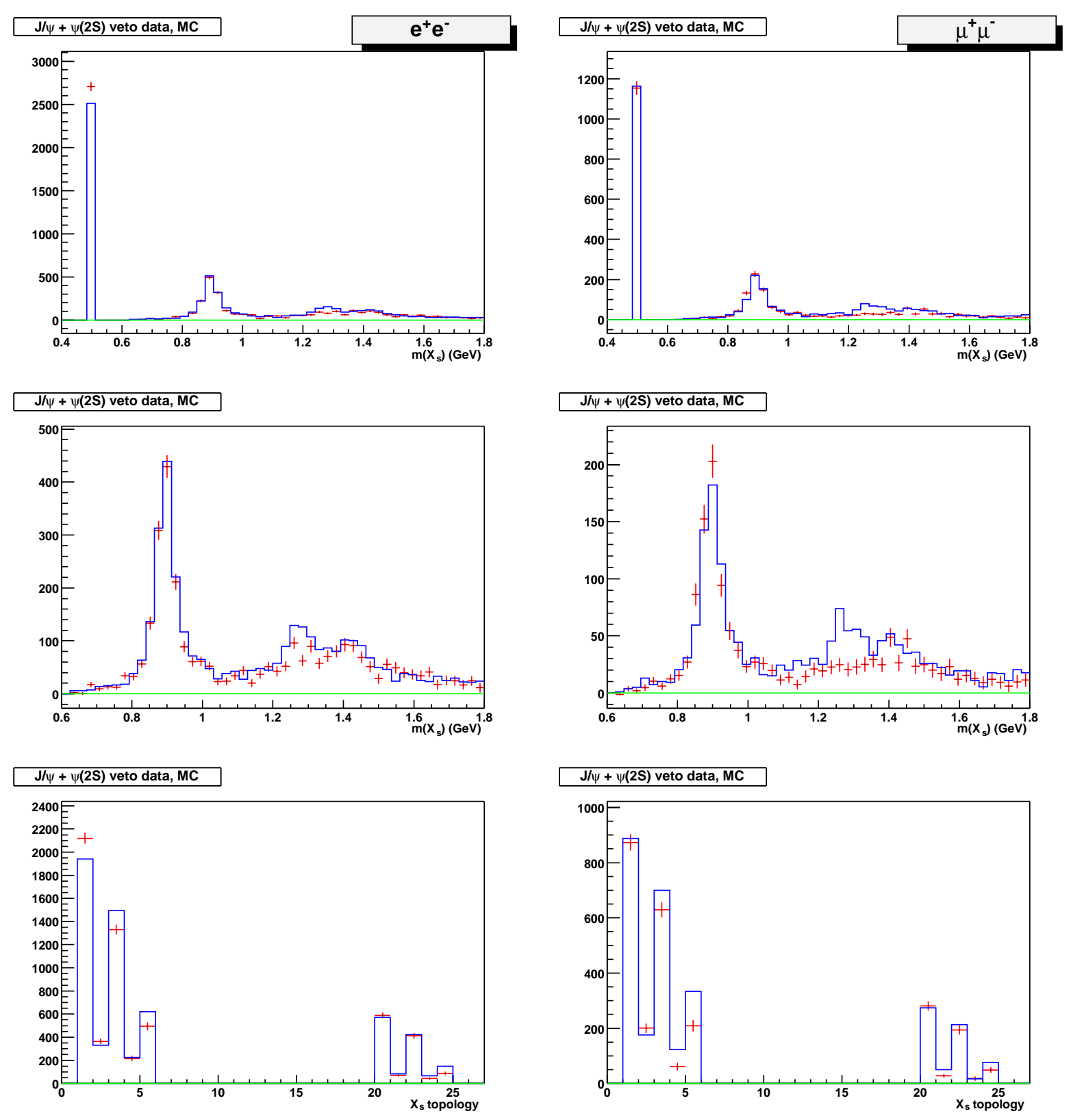

Figure 51: Distributions of hadronic invariant mass and final state topology (see Table 6) for $B \rightarrow X_{s} \ell^{+} \ell^{-}$candidates in the charmonium veto sample for electrons (left) and muons (right) after background subtraction. The points represent the data and the histograms the MC, scaled to correspond to an integrated luminosity of $81.9 \mathrm{fb}^{-1}$. 
Table 7: Ratio of background-subtracted $B \rightarrow J / \psi X$ yields for data and MC in the different topologies for the electron channels.

\begin{tabular}{lccc}
\hline \hline$X_{s}$ Topology & Data/MC & Data/MC(corrected) & MC correction \\
\hline$K$ & $1.118 \pm 0.036$ & $0.991 \pm 0.061$ & $1.128 \pm 0.059$ \\
$K \pi^{0}$ & $1.16 \pm 0.13$ & $1.08 \pm 0.16$ & $1.07 \pm 0.10$ \\
$K \pi$ & $1.013 \pm 0.048$ & $1.00 \pm 0.09$ & $1.01 \pm 0.07$ \\
$K \pi \pi^{0}$ & $1.07 \pm 0.24$ & & \\
$K \pi \pi$ & $0.67 \pm 0.08$ & & \\
\hline$K_{S}^{0}$ & $1.107 \pm 0.069$ & $1.14 \pm 0.10$ & $0.971 \pm 0.058$ \\
$K_{S}^{0} \pi^{0}$ & $0.79 \pm 0.20$ & $0.78 \pm 0.21$ & $1.01 \pm 0.07$ \\
$K_{S}^{0} \pi$ & $1.01 \pm 0.09$ & $0.94 \pm 0.12$ & $1.07 \pm 0.10$ \\
$K_{S}^{0} \pi \pi^{0}$ & $0.64 \pm 0.35$ & & \\
$K_{S}^{0} \pi \pi$ & $0.49 \pm 0.16$ & & \\
\hline All above $J / \psi X$ modes & $1.035 \pm 0.024$ & & \\
$J / \psi X(0$ or $1 \pi)$ & $1.078 \pm 0.025$ & $1.008 \pm 0.041$ & \\
\hline
\end{tabular}

tween data and $\mathrm{MC}$ is most useful in the lower multiplicity $J / \psi K$ and $J / \psi K^{*}$ final states. Tables 7 and 8 indicate good agreement between data and MC. In the third column of these tables, the MC yields have been corrected to account for differences between branching fractions used in the MC generator and those listed in the PDG [2]. (Here, we assumed that the $K \pi$ and $K \pi^{0}$ modes are dominated by the $K^{*}$ resonance.) Tables 11 and 12 compare the branching fractions used in the MC generator with those from the PDG [2]. The exclusive decay modes listed in the table are supplemented with inclusive quark processes hadronized via Jetset, the result of this process is tuned such as to reproduce the fully inclusive branching fraction, as shown in the last row of Tables 11 and 12 . It should be noted that the corrected ratio data/MC for combined 0 and 1 pion modes given in Tables $7-10$ is computed assuming the total uncertainties in the individual modes are uncorrelated. The uncertainty in that combined mode ratio is thus a lower estimate. 

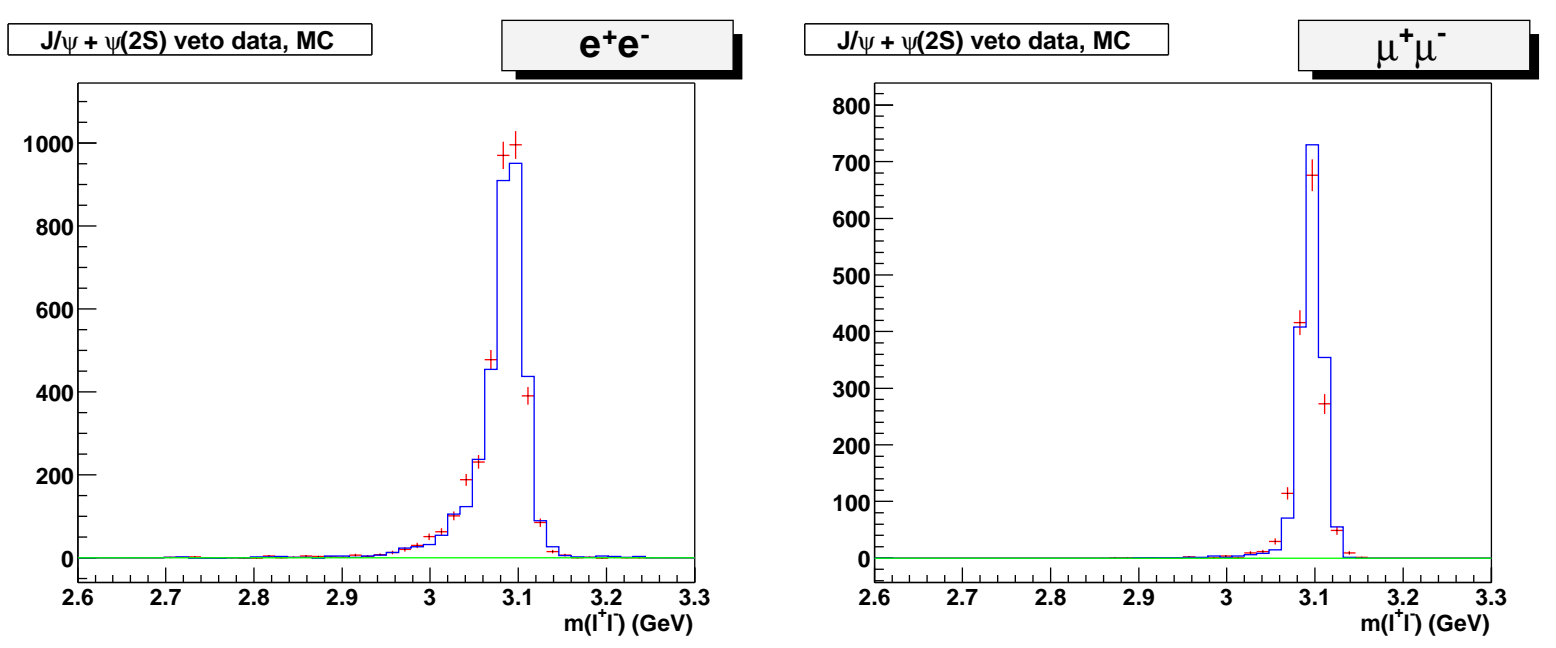

$\mathbf{J} / \psi+\psi(2 \mathbf{S})$ veto data, $\mathbf{M C}$

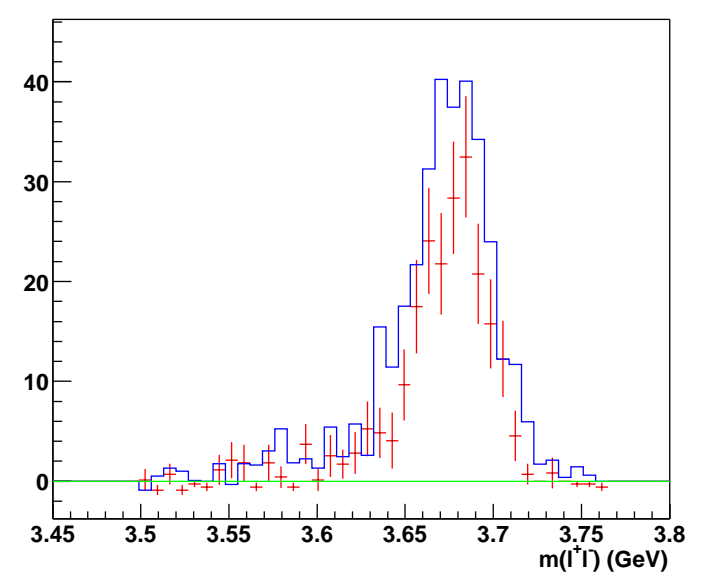

$\mathrm{J} / \psi+\psi(\mathbf{2 S})$ veto data, $\mathbf{M C}$

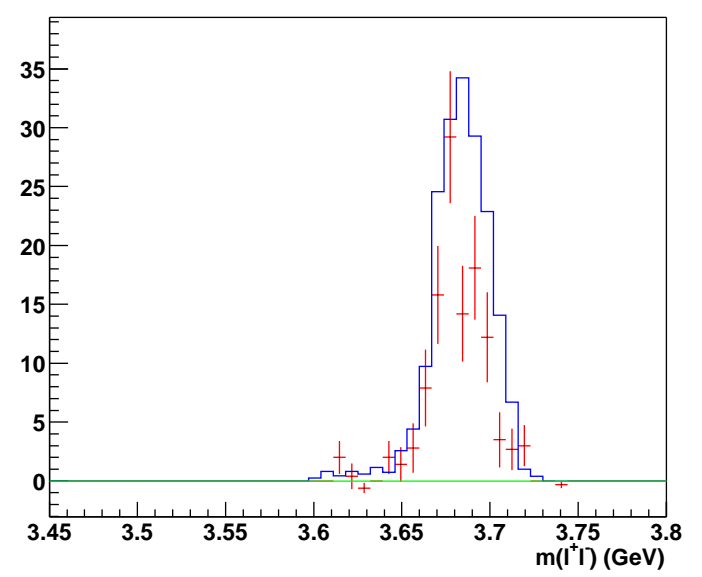

Figure 52: Distributions of dilepton invariant mass for $B \rightarrow X_{s} \ell^{+} \ell^{-}$candidates in the charmonium veto sample for electrons (left) and muons (right) after likelihood ratio cuts and background subtraction. The top and bottom rows are for the $J / \psi$ and $\psi(2 S)$ mass regions, respectively. The points represent the data and the histograms the $\mathrm{MC}$, scaled to correspond to an integrated luminosity of $81.9 \mathrm{fb}^{-1}$. 

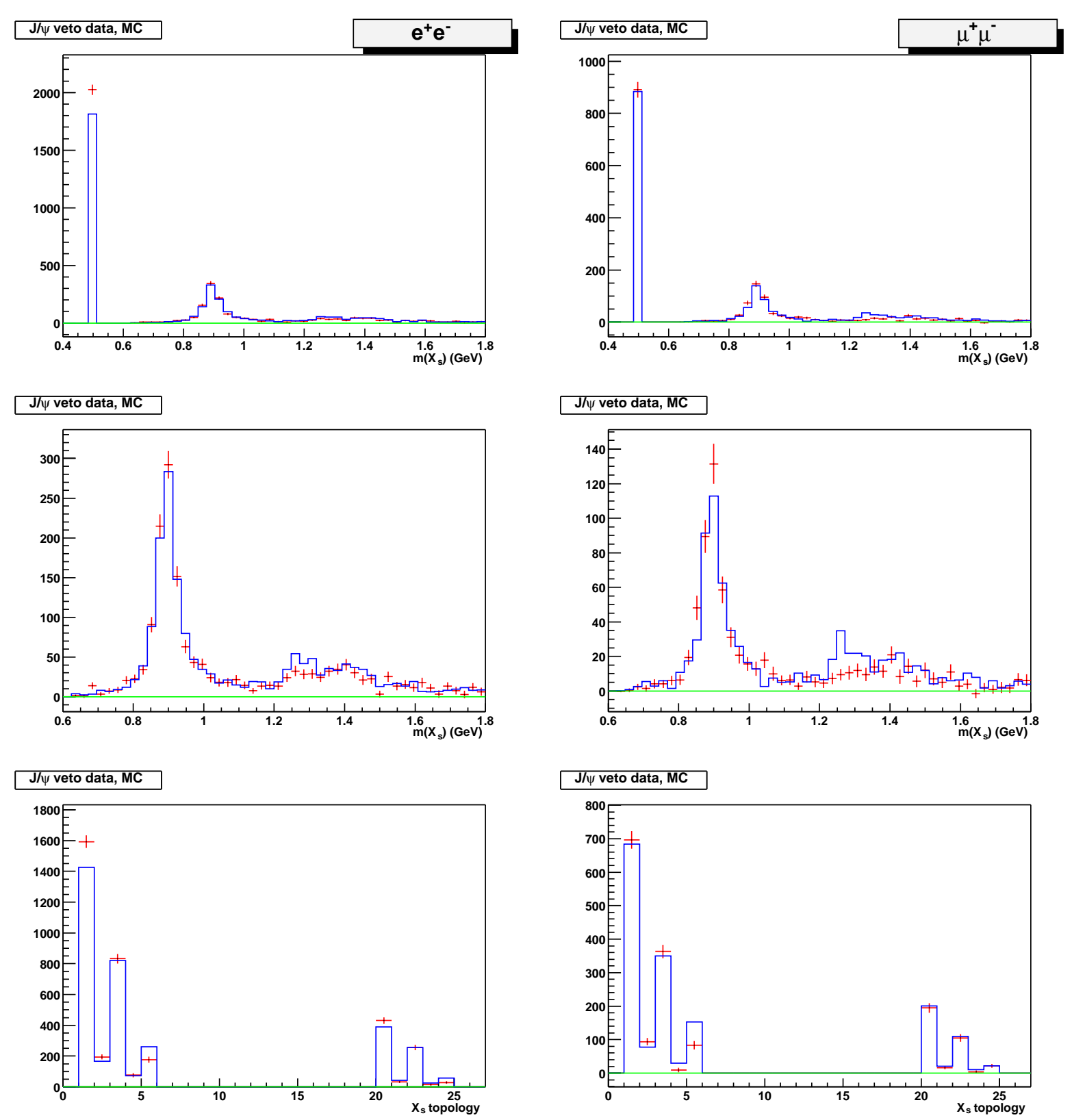

Figure 53: Distributions of hadronic invariant mass and final state topology (see Table 6) for $B \rightarrow X_{s} \ell^{+} \ell^{-}$candidates in the $B \rightarrow J / \psi X$ veto sample for electrons (left) and muons (right) after likelihood ratio cuts and background subtraction. The points represent the data and the histograms the MC, scaled to correspond to an integrated luminosity of $81.9 \mathrm{fb}^{-1}$. 

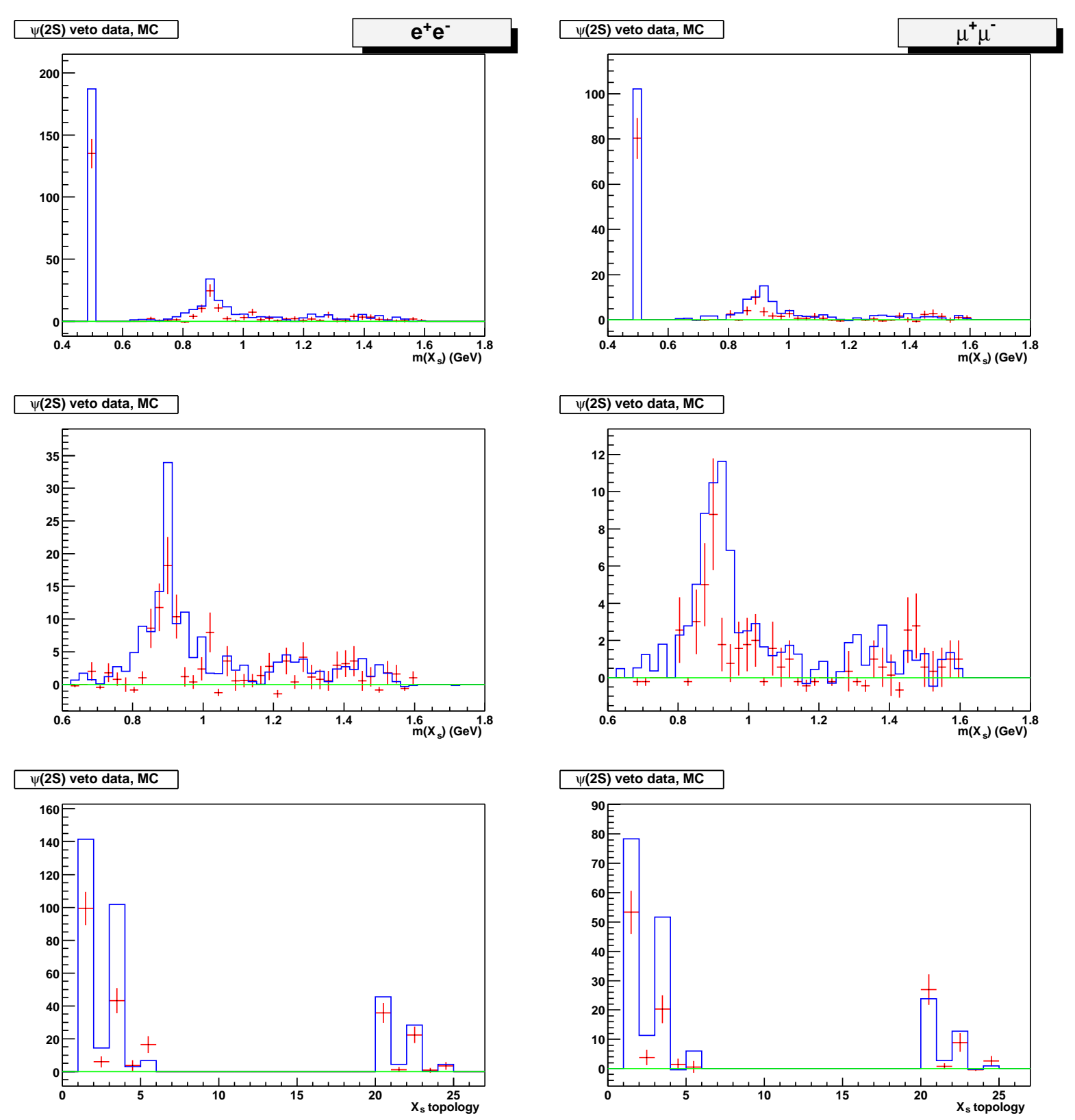

Figure 54: Distributions of hadronic invariant mass and final state topology (see Table 6) for $B \rightarrow X_{s} \ell^{+} \ell^{-}$candidates in the $B \rightarrow \psi(2 S) X$ veto sample for electrons (left) and muons (right) after likelihood ratio cuts and background subtraction. The points represent the data and the histograms the MC, scaled to correspond to an integrated luminosity of $81.9 \mathrm{fb}^{-1}$. 

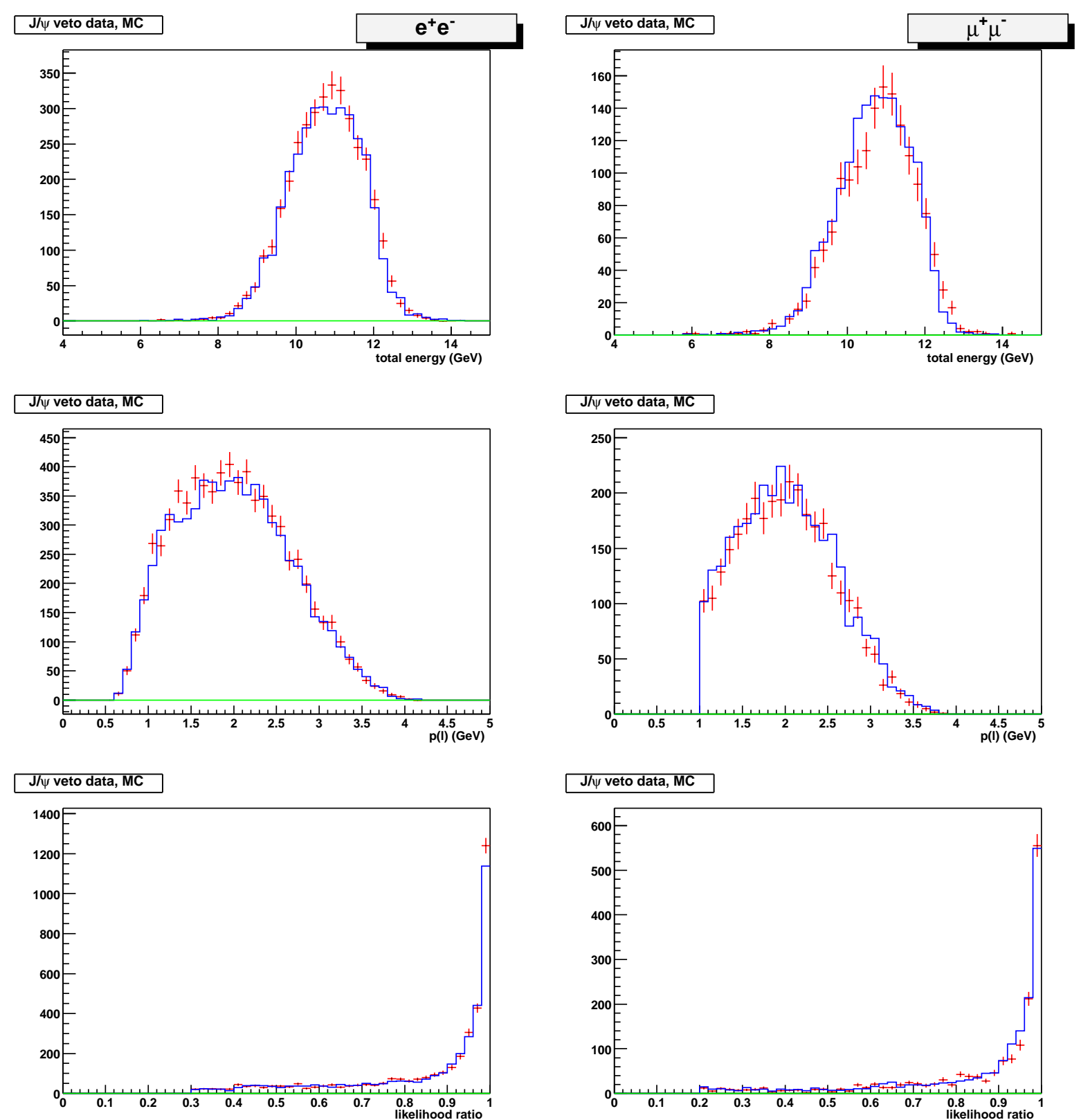

Figure 55: Distributions of total event energy, lepton momentum (both in the lab frame), and likelihood ratio for $B \rightarrow X_{s} \ell^{+} \ell^{-}$candidates in the $B \rightarrow J / \psi X$ veto sample for electrons (left) and muons (right) after likelihood ratio cuts and background subtraction. The points represent the data and the histograms the MC, scaled to correspond to an integrated luminosity of $81.9 \mathrm{fb}^{-1}$. 

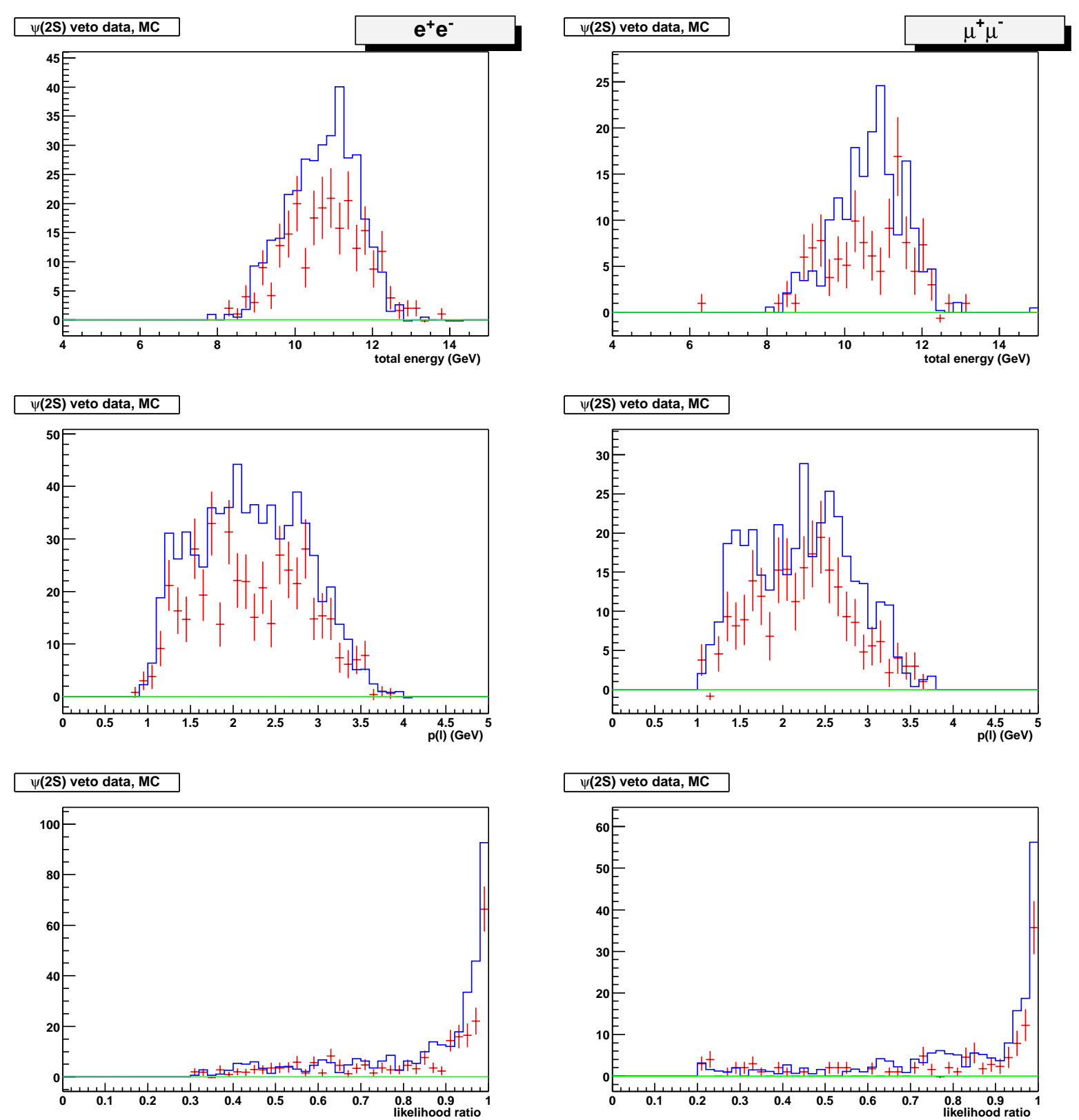

Figure 56: Distributions of total event energy, lepton momentum (both in the lab frame), and likelihood ratio for $B \rightarrow X_{s} \ell^{+} \ell^{-}$candidates in the $B \rightarrow \psi(2 S) X$ veto sample for electrons (left) and muons (right) after likelihood ratio cuts and background subtraction. The points represent the data and the histograms the $\mathrm{MC}$, scaled to correspond to an integrated luminosity of $81.9 \mathrm{fb}^{-1}$. 
Table 8: Ratio of background-subtracted $B \rightarrow J / \psi X$ yields for data and MC in the different topologies for the muon channels.

\begin{tabular}{lccc}
\hline \hline$X_{s}$ Topology & Data/MC & Data/MC(corrected) & MC correction \\
\hline$K$ & $1.018 \pm 0.048$ & $0.910 \pm 0.064$ & $1.118 \pm 0.059$ \\
$K \pi^{0}$ & $1.21 \pm 0.18$ & $1.14 \pm 0.20$ & $1.07 \pm 0.10$ \\
$K \pi$ & $1.040 \pm 0.072$ & $1.04 \pm 0.10$ & $1.00 \pm 0.07$ \\
$K \pi \pi^{0}$ & $0.31 \pm 0.24$ & & \\
$K \pi \pi$ & $0.54 \pm 0.09$ & & \\
\hline$K_{S}^{0}$ & $0.97 \pm 0.09$ & $1.01 \pm 0.11$ & $0.963 \pm 0.058$ \\
$K_{S}^{0} \pi^{0}$ & $0.78 \pm 0.26$ & $0.78 \pm 0.27$ & $1.00 \pm 0.07$ \\
$K_{S}^{0} \pi$ & $0.96 \pm 0.13$ & $0.90 \pm 0.15$ & $1.07 \pm 0.10$ \\
$K_{S}^{0} \pi \pi^{0}$ & $0.32 \pm 0.42$ & & \\
$K_{S}^{0} \pi \pi$ & $0.99 \pm 0.33$ & & \\
\hline All above $J / \psi X$ modes & $0.957 \pm 0.032$ & & \\
$J / \psi X(0$ or $1 \pi)$ & $1.019 \pm 0.034$ & $0.961 \pm 0.045$ & \\
\hline
\end{tabular}

Table 9: Ratio of background-subtracted $B \rightarrow \psi(2 S) X$ yields for data and $\mathrm{MC}$ in the different topologies for the electron channels.

\begin{tabular}{lccc}
\hline \hline$X_{s}$ Topology & Data/MC & Data/MC(corrected) & MC correction \\
\hline$K$ & $0.70 \pm 0.08$ & $0.90 \pm 0.15$ & $0.78 \pm 0.08$ \\
$K \pi^{0}$ & $0.41 \pm 0.27$ & $0.55 \pm 0.38$ & $0.75 \pm 0.18$ \\
$K \pi$ & $0.42 \pm 0.08$ & $0.65 \pm 0.17$ & $0.65 \pm 0.11$ \\
$K \pi \pi^{0}$ & $1.3 \pm 1.4$ & & \\
$K \pi \pi$ & $2.4 \pm 1.5$ & & \\
\hline$K_{S}^{0}$ & $0.79 \pm 0.16$ & $1.17 \pm 0.32$ & $0.67 \pm 0.12$ \\
$K_{S}^{0} \pi^{0}$ & $0.27 \pm 0.35$ & $0.41 \pm 0.55$ & $0.65 \pm 0.11$ \\
$K_{S}^{0} \pi$ & $0.79 \pm 0.22$ & $1.06 \pm 0.39$ & $0.75 \pm 0.18$ \\
$K_{S}^{0} \pi \pi^{0}$ & insuff.stats. & & \\
$K_{S}^{0} \pi \pi$ & $0.79 \pm 0.65$ & & \\
\hline All above $\psi(2 S) X$ modes & $0.663 \pm 0.056$ & & \\
$\psi(2 S) X(0$ or $1 \pi)$ & $0.619 \pm 0.053$ & $0.86 \pm 0.10$ & \\
\hline & & &
\end{tabular}


Table 10: Ratio of background-subtracted $B \rightarrow \psi(2 S) X$ yields for data and $\mathrm{MC}$ in the different topologies for the muon channels.

\begin{tabular}{lccc}
\hline \hline$X_{s}$ Topology & Data/MC & Data/MC(corrected) & MC correction \\
\hline$K$ & $0.68 \pm 0.11$ & $0.91 \pm 0.21$ & $0.75 \pm 0.12$ \\
$K \pi^{0}$ & $0.33 \pm 0.24$ & $0.47 \pm 0.36$ & $0.72 \pm 0.19$ \\
$K \pi$ & $0.39 \pm 0.10$ & $0.63 \pm 0.21$ & $0.62 \pm 0.13$ \\
$K \pi \pi^{0}$ & insuff.stats. & & \\
$K \pi \pi$ & $0.10 \pm 0.34$ & & $0.64 \pm 0.14$ \\
\hline$K_{S}^{0}$ & $1.13 \pm 0.28$ & $1.76 \pm 0.58$ & $0.62 \pm 0.13$ \\
$K_{S}^{0} \pi^{0}$ & $0.28 \pm 0.40$ & $0.46 \pm 0.65$ & $0.72 \pm 0.19$ \\
$K_{S}^{0} \pi$ & $0.70 \pm 0.30$ & $0.97 \pm 0.49$ & \\
$K_{S}^{0} \pi \pi^{0}$ & insuff.stats. & & \\
$K_{S}^{0} \pi \pi$ & insuff.stats. & & \\
\hline All above $\psi(2 S) X$ modes & $0.633 \pm 0.071$ & & \\
$\psi(2 S) X(0$ or $1 \pi)$ & $0.631 \pm 0.071$ & $0.91 \pm 0.14$ & \\
\hline
\end{tabular}

\section{2 $X_{s} e^{ \pm} \mu^{\mp}$ sample}

Since $X_{s} e^{ \pm} \mu^{\mp}$ combinations cannot result from a $B$ decay, they do not peak in $m_{E S}$ and therefore, are a good control sample of combinatorial background events. The sample is collected in parallel with the standard analysis described above, the only difference being the requirement for a different flavor of the dilepton candidates (1 $e$ and $1 \mu$ ). For the charmonium veto and the calculation of the likelihood ratio, we treat the $X_{s} e^{ \pm} \mu^{\mp}$ candidate as if it were an $X_{s} \mu^{+} \mu^{-}$candidate. Final states with three pions are rejected and nominal likelihood ratio cuts are applied.

Distributions of $m_{\mathrm{ES}}$ for data and MC, fitted with an Argus function, are shown in Fig. 57. The fitted parameters obtained from the two distributions are consistent within their errors. Other data and MC comparisons are shown in Fig. 58. The agreement is good and the ratio between the number of events in the data and $\mathrm{MC}$ is $0.986 \pm 0.050$, which shows that the combinatorial background is simulated accurately and no additional scaling is necessary. It should be noted that the 
Table 11: Branching fractions for $B$ decays to $J / \psi$ in SP4 MC (EvtGen) and data (PDG) [2]. The last entry gives the fully inclusive branching fraction at the $\Upsilon(4 S)$ where the MC uncertainty corresponds to the statistical uncertainty obtained in a large sample of generated events.

\begin{tabular}{lcc}
\hline \hline Decay mode & EvtGen MC & PDG \\
\hline$B^{0} \rightarrow J / \psi K^{0}$ & $9.0 \times 10^{-4}$ & $(8.7 \pm 0.5) \times 10^{-4}$ \\
$B^{0} \rightarrow J / \psi K^{* 0}$ & $1.3 \times 10^{-3}$ & $(1.31 \pm 0.09) \times 10^{-3}$ \\
$B^{0} \rightarrow J / \psi K^{+} \pi^{-}$ & $2.0 \times 10^{-4}$ & \\
$B^{0} \rightarrow J / \psi K^{0} \pi^{0}$ & $1.0 \times 10^{-4}$ & \\
$B^{0} \rightarrow J / \psi \phi K^{0}$ & $9.0 \times 10^{-5}$ & $\left(8.8_{-3.3}^{+3.7}\right) \times 10^{-5}$ \\
$B^{0} \rightarrow J / \psi K_{1}(1270)^{0}$ & $1.5 \times 10^{-3}$ & $(1.3 \pm 0.5) \times 10^{-3}$ \\
$B^{0} \rightarrow J / \psi K_{1}(140)^{0}$ & $1.0 \times 10^{-4}$ & \\
$B^{0} \rightarrow J / \psi K_{2}^{*}(1430)^{0}$ & $5.0 \times 10^{-4}$ & \\
$B^{0} \rightarrow J / \psi K^{0} \pi^{+} \pi^{-}$ & & $(1.0 \pm 0.4) \times 10^{-4}$ \\
$B^{0} \rightarrow J / \psi K^{*+} \pi^{-}$ & & $(0.8 \pm 0.4) \times 10^{-3}$ \\
$B^{0} \rightarrow J / \psi K^{* 0} \pi^{+} \pi^{-}$ & & $(6.6 \pm 2.2) \times 10^{-4}$ \\
$B^{0} \rightarrow J / \psi \pi^{0}$ & $2.0 \times 10^{-5}$ & $(2.1 \pm 0.5) \times 10^{-4}$ \\
$B^{0} \rightarrow J / \psi \rho^{0}$ & $3.0 \times 10^{-5}$ & $<2.5 \times 10^{-4}$ \\
$B^{0} \rightarrow J / \psi \omega$ & $3.0 \times 10^{-5}$ & $<2.7 \times 10^{-4}$ \\
\hline$B^{+} \rightarrow J / \psi K^{+}$ & $9.0 \times 10^{-4}$ & $(1.01 \pm 0.05) \times 10^{-3}$ \\
$B^{+} \rightarrow J / \psi K^{*+}$ & $1.3 \times 10^{-3}$ & $(1.39 \pm 0.13) \times 10^{-3}$ \\
$B^{+} \rightarrow J / \psi K^{+} \pi^{0}$ & $1.0 \times 10^{-4}$ & \\
$B^{+} \rightarrow J / \psi K^{0} \pi^{+}$ & $2.0 \times 10^{-4}$ & \\
$B^{+} \rightarrow J / \psi \phi K^{+}$ & $9.0 \times 10^{-5}$ & $\left(8.8_{-3.3}^{+3.7}\right) \times 10^{-5}$ \\
$B^{+} \rightarrow J / \psi K_{1}(1270)^{+}$ & $1.5 \times 10^{-3}$ & $(1.8 \pm 0.5) \times 10^{-3}$ \\
$B^{+} \rightarrow J / \psi K_{1}(1400)^{+}$ & $1.0 \times 10^{-4}$ & $<5 \times 10^{-4}$ \\
$B^{+} \rightarrow J / \psi K_{2}^{*}(1430)^{+}$ & $5.0 \times 10^{-4}$ & \\
$B^{+} \rightarrow J / \psi K^{+} \pi^{+} \pi^{-}$ & & $(1.4 \pm 0.6) \times 10^{-3}$ \\
$B^{+} \rightarrow J / \psi \pi^{+}$ & $4.0 \times 10^{-5}$ & $(4.2 \pm 0.7) \times 10^{-5}$ \\
$B^{+} \rightarrow J / \psi \rho^{+}$ & $6.0 \times 10^{-5}$ & $<7.7 \times 10^{-4}$ \\
\hline$B \rightarrow J / \psi X$ & $(1.10 \pm 0.02) \times 10^{-2}$ & $(1.15 \pm 0.06) \times 10^{-2}$ \\
\hline \hline
\end{tabular}


Table 12: Branching fractions for $B$ decays to $\psi(2 S)$ in SP4 MC (EvtGen) and data (PDG) [2]. The last entry gives the fully inclusive branching fraction at the $\Upsilon(4 S)$ where the MC uncertainty corresponds to the statistical uncertainty obtained in a large sample of generated events.

\begin{tabular}{lcc}
\hline \hline Decay mode & EvtGen MC & PDG \\
\hline$B^{0} \rightarrow \psi(2 S) K^{0}$ & $6.2 \times 10^{-4}$ & $(5.7 \pm 1.0) \times 10^{-4}$ \\
$B^{0} \rightarrow \psi(2 S) K^{* 0}$ & $9.0 \times 10^{-4}$ & $(8.0 \pm 1.3) \times 10^{-4}$ \\
$B^{0} \rightarrow \psi(2 S) K^{+} \pi^{-}$ & $4.0 \times 10^{-4}$ & \\
$B^{0} \rightarrow \psi(2 S) K^{0} \pi^{0}$ & $2.0 \times 10^{-4}$ & \\
$B^{0} \rightarrow \psi(2 S) K^{0} \pi^{+} \pi^{-}$ & $2.0 \times 10^{-4}$ & \\
$B^{0} \rightarrow \psi(2 S) K^{0} \pi^{0} \pi^{0}$ & $1.0 \times 10^{-4}$ & \\
$B^{0} \rightarrow \psi(2 S) K^{+} \pi^{-} \pi^{0}$ & $1.0 \times 10^{-4}$ & \\
$B^{0} \rightarrow \psi(2 S) K_{1}(1270)^{0}$ & $4.0 \times 10^{-4}$ & \\
\hline$B^{+} \rightarrow \psi(2 S) K^{+}$ & $6.2 \times 10^{-4}$ & $(6.6 \pm 0.6) \times 10^{-4}$ \\
$B^{+} \rightarrow \psi(2 S) K^{*+}$ & $9.0 \times 10^{-4}$ & $(9.2 \pm 2.2) \times 10^{-4}$ \\
$B^{+} \rightarrow \psi(2 S) K^{+} \pi^{0}$ & $2.0 \times 10^{-4}$ & \\
$B^{+} \rightarrow \psi(2 S) K^{0} \pi^{+}$ & $4.0 \times 10^{-4}$ & \\
$B^{+} \rightarrow \psi(2 S) K^{+} \pi^{+} \pi^{-}$ & $2.0 \times 10^{-4}$ & $(1.9 \pm 1.2) \times 10^{-3}$ \\
$B^{+} \rightarrow \psi(2 S) K^{+} \pi^{0} \pi^{0}$ & $1.0 \times 10^{-4}$ & \\
$B^{+} \rightarrow \psi(2 S) K^{0} \pi^{+} \pi^{0}$ & $1.0 \times 10^{-4}$ & \\
$B^{+} \rightarrow \psi(2 S) K_{1}(1270)^{+}$ & $4.0 \times 10^{-4}$ & \\
\hline$B \rightarrow \psi(2 S) X$ & $(3.2 \pm 0.1) \times 10^{-3}$ & $(3.5 \pm 0.5) \times 10^{-3}$ \\
\hline \hline
\end{tabular}

Table 13: Branching fractions for charmonium decays in SP4 MC (EvtGen) and data (PDG) [2].

\begin{tabular}{lcc}
\hline \hline Decay mode & EvtGen MC & PDG \\
\hline$J / \psi \rightarrow e^{+} e^{-}$ & $5.90 \times 10^{-2}$ & $(5.93 \pm 0.10) \times 10^{-2}$ \\
$J / \psi \rightarrow \mu^{+} \mu^{-}$ & $5.90 \times 10^{-2}$ & $(5.88 \pm 0.10) \times 10^{-2}$ \\
\hline$\psi(2 S) \rightarrow e^{+} e^{-}$ & $1.00 \times 10^{-2}$ & $(0.73 \pm 0.04) \times 10^{-2}$ \\
$\psi(2 S) \rightarrow \mu^{+} \mu^{-}$ & $1.00 \times 10^{-2}$ & $(0.70 \pm 0.09) \times 10^{-2}$ \\
\hline \hline
\end{tabular}


fit for the signal yield does not rely on this sample to describe the shape of the combinatorial background.

\subsection{Off-resonance sample}

In order to cross-check the combinatorial background contribution due to continuum events, as obtained from MC, we use off-resonance data, taken $40 \mathrm{MeV}$ below the $\Upsilon(4 S)$ peak. When comparing the data with MC, the reconstructed $m_{E S}$ value in the $\mathrm{MC}$ is decreased by $20 \mathrm{MeV}$ and all analysis cuts are applied. Data and MC are compared in Figs. 59 and 60. Agreement is good but statistics are poor. The ratio between the number of events in the data and $\mathrm{MC}$ is $1.78 \pm 0.86$ in the electron channel and $0.57 \pm 0.42$ in the muon channel.

\subsection{On-resonance sideband sample}

On-resonance sideband data, with $m_{E S}<5.27 \mathrm{GeV}$ provides another check of the combinatorial background. Comparison between data and MC shows good agreement, see Figs. 61-6.4. The ratio between the number of events in the data and $\mathrm{MC}$ is $0.96 \pm 0.08$ in the electron channel and $1.17 \pm 0.13$ in the muon channel. 


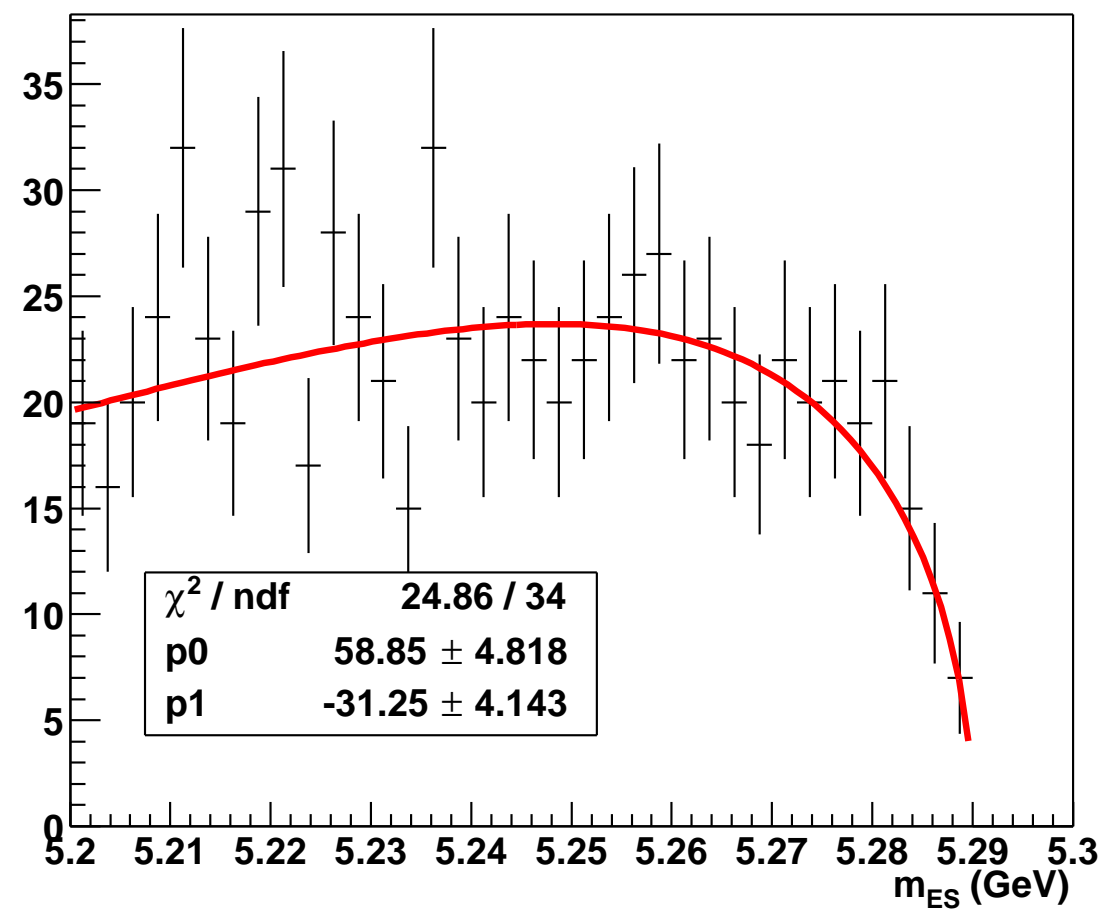

\section{e $\mu$ MC, argus fit}

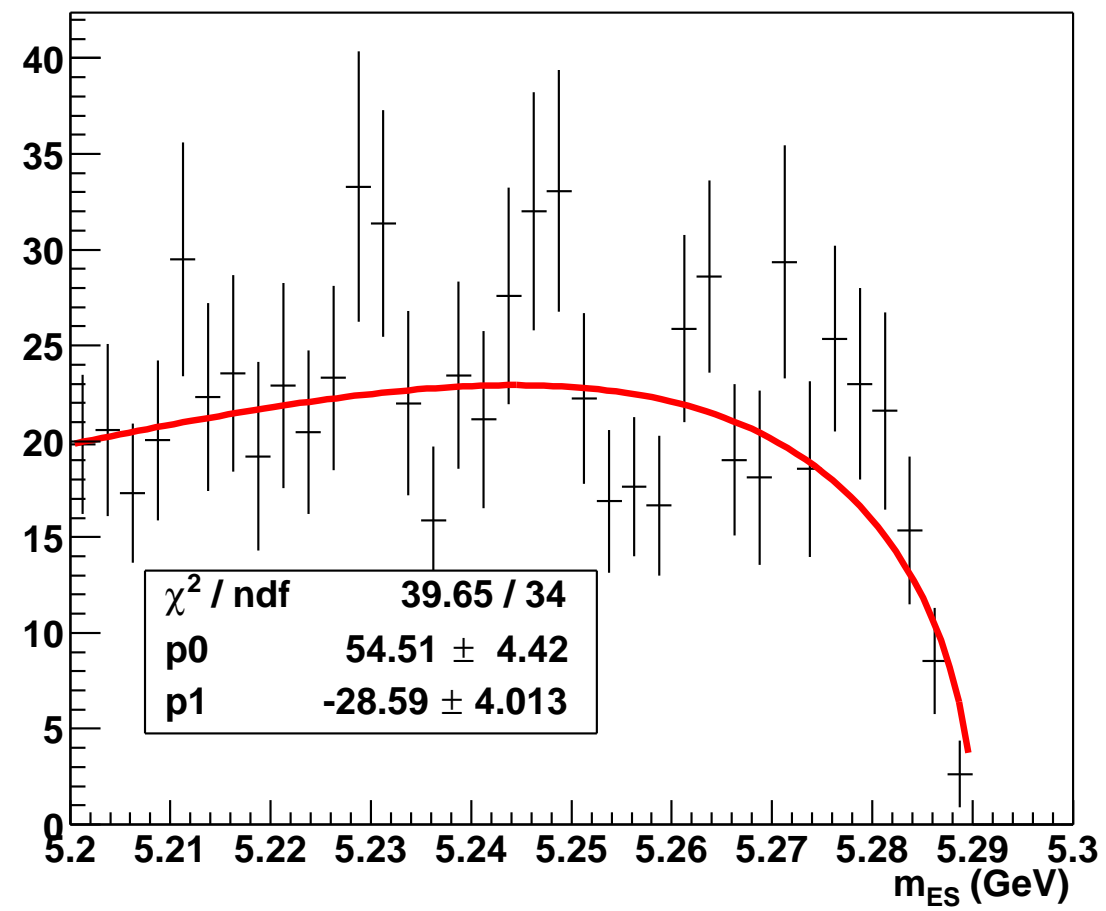

Figure 57: Distributions of $m_{E S}$ for $B$ decay candidates in the $B \rightarrow X_{s} e^{ \pm} \mu^{\mp}$ sample for data (top) and MC (bottom). The MC distribution has been scaled to correspond $166 \mathrm{an}$ integrated luminosity of $81.9 \mathrm{fb}^{-1}$. 

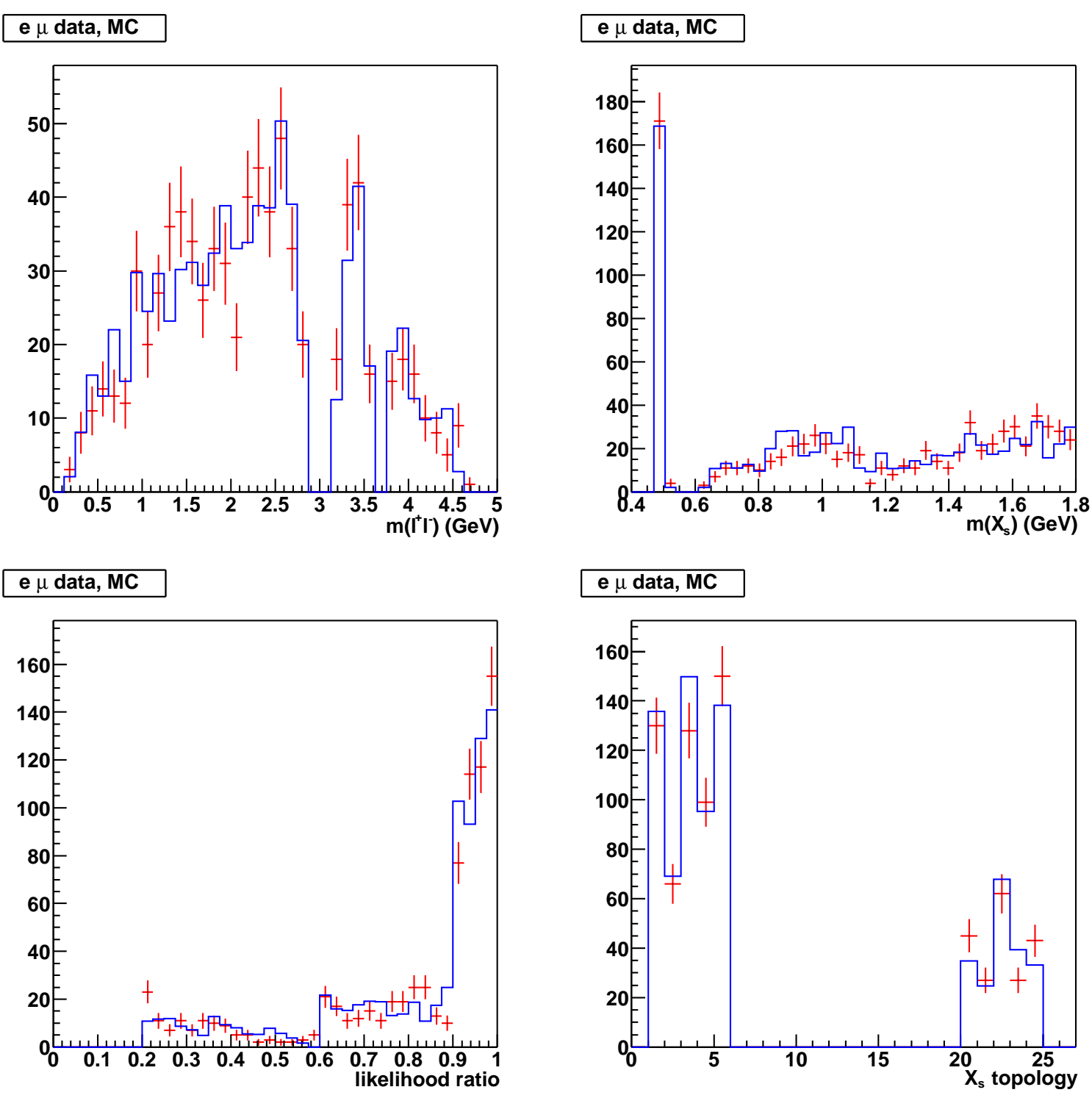

Figure 58: Distributions of dilepton mass, hadronic mass, likelihood ratio and final state topology for $B$ decay candidates in the $B \rightarrow X_{s} e^{ \pm} \mu^{\mp}$ sample. The points represent the data and the histograms the MC, scaled to correspond to an integrated luminosity of $81.9 \mathrm{fb}^{-1}$. 

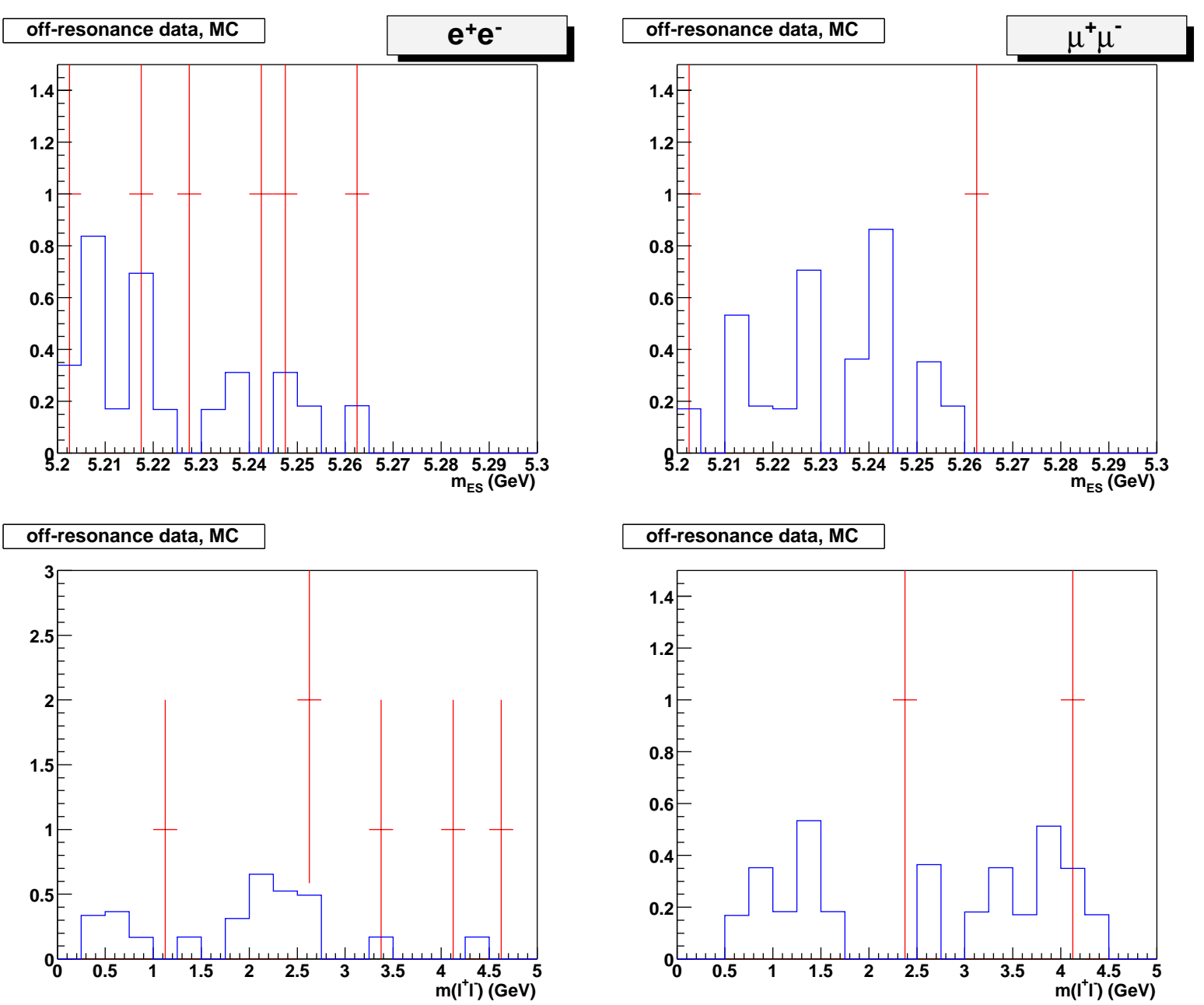

Figure 59: Distributions of $m_{E S}$ and dilepton mass for $B \rightarrow X_{s} \ell^{+} \ell^{-}$candidates in the off-resonance data sample for electrons (left) and muons (right). The points represent the data and the histograms the $\mathrm{MC}$, scaled to correspond to an integrated luminosity of $9.6 \mathrm{fb}^{-1}$. 

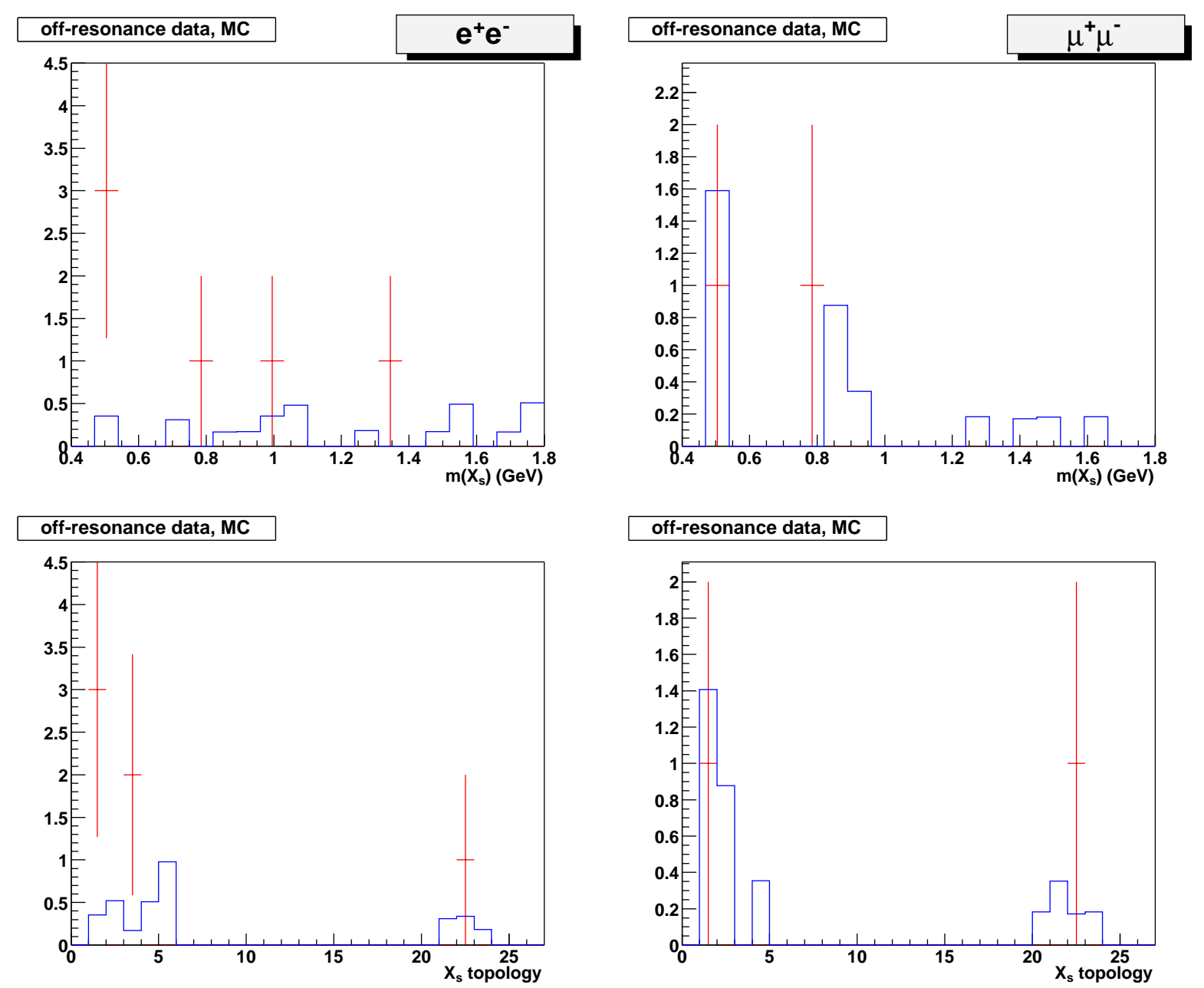

Figure 60: Distributions of hadronic mass and final state topology for $B \rightarrow$ $X_{s} \ell^{+} \ell^{-}$candidates in the off-resonance data sample for electrons (left) and muons (right). The points represent the data and the histograms the MC, scaled to correspond to an integrated luminosity of $9.6 \mathrm{fb}^{-1}$. 

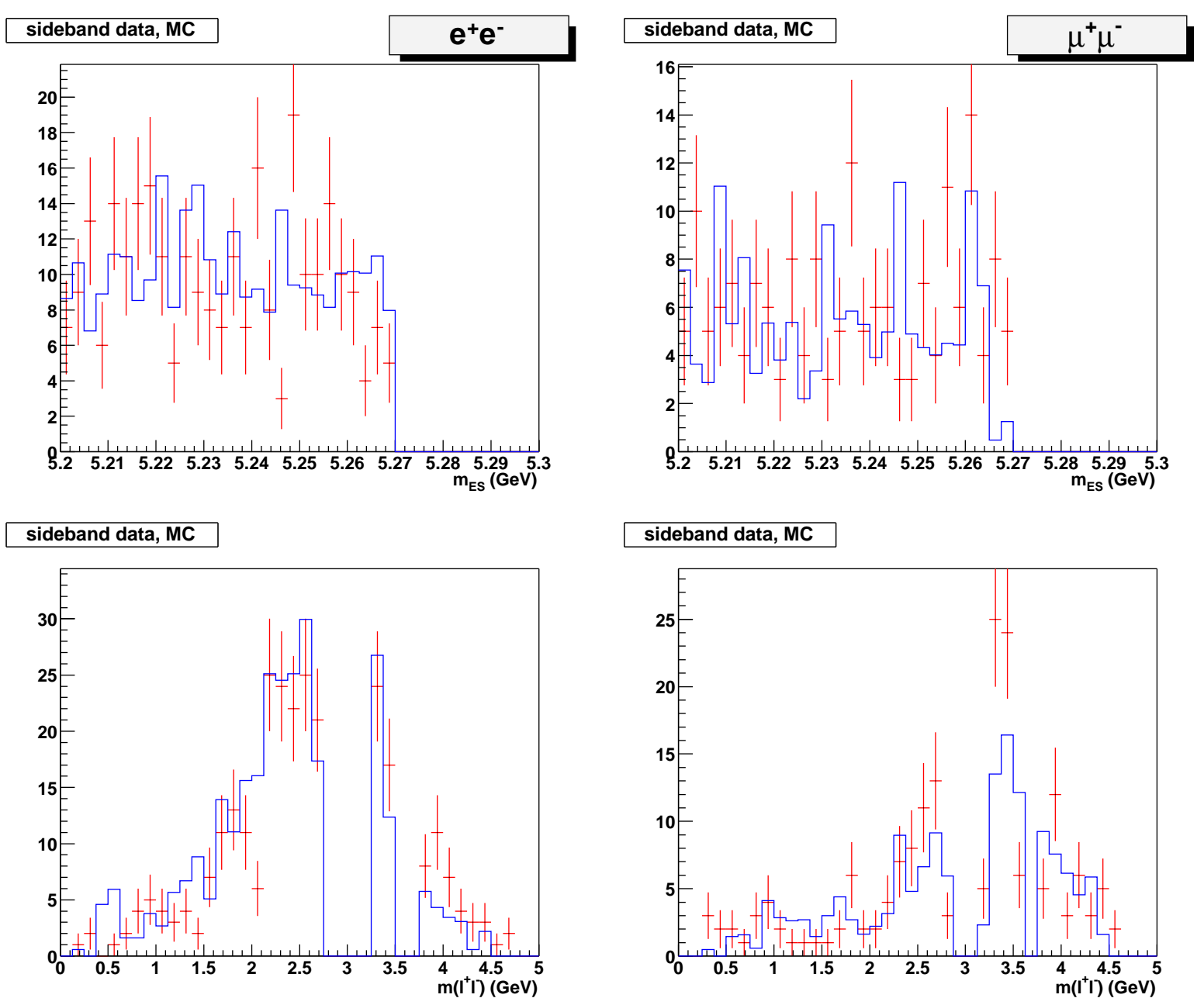

Figure 61: Distributions of $m_{E S}$ and dilepton mass for $B \rightarrow X_{s} \ell^{+} \ell^{-}$candidates in the sideband data sample for electrons (left) and muons (right). The points represent the data and the histograms the $\mathrm{MC}$, scaled to correspond to an integrated luminosity of $81.9 \mathrm{fb}^{-1}$. 

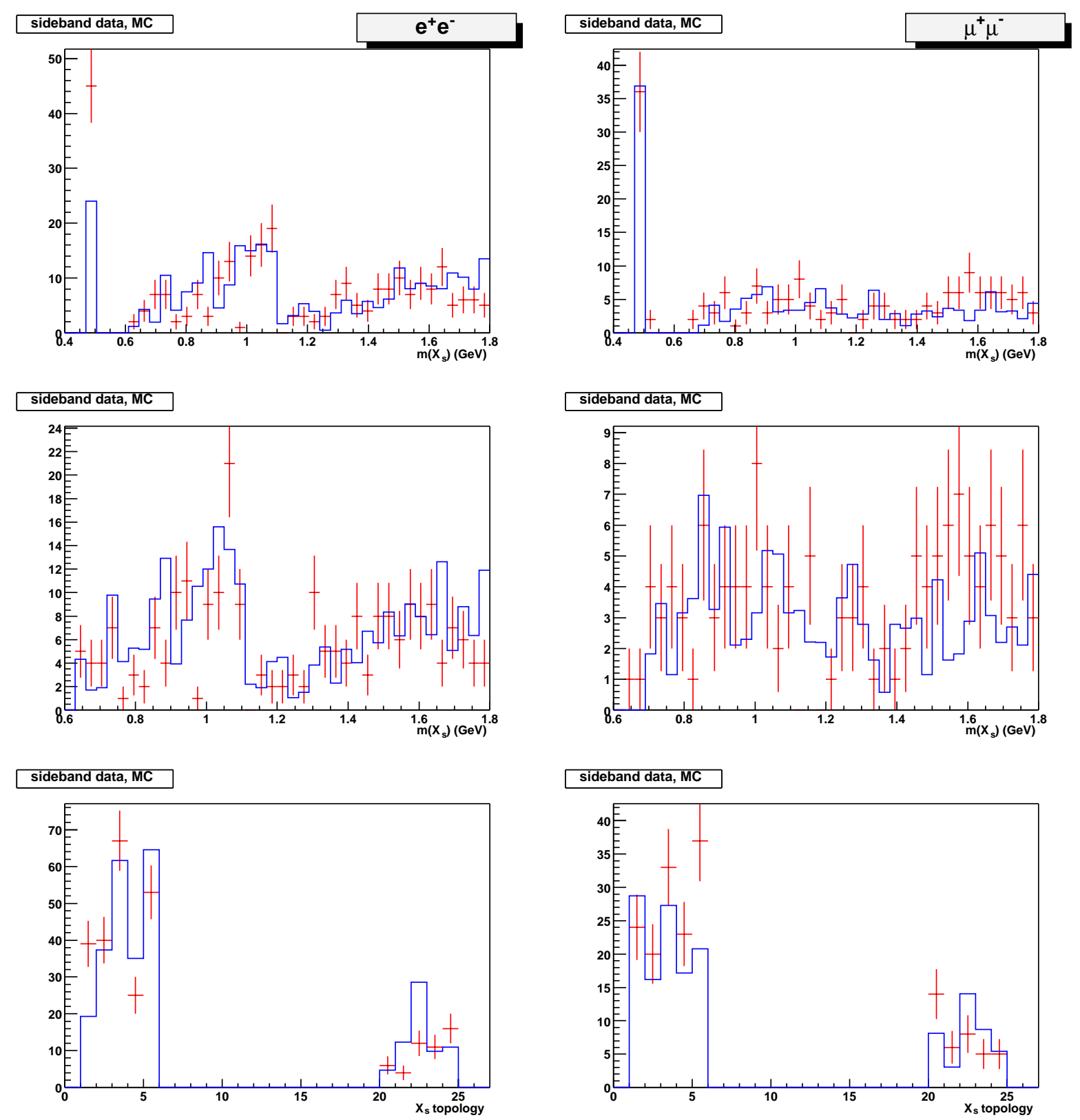

Figure 62: Distributions of hadronic mass and final state topology for $B \rightarrow$ $X_{s} \ell^{+} \ell^{-}$candidates in the sideband data sample for electrons (left) and muons (right). The points represent the data and the histograms the MC, scaled to correspond to an integrated luminosity of $81.9 \mathrm{fb}^{-1}$. 

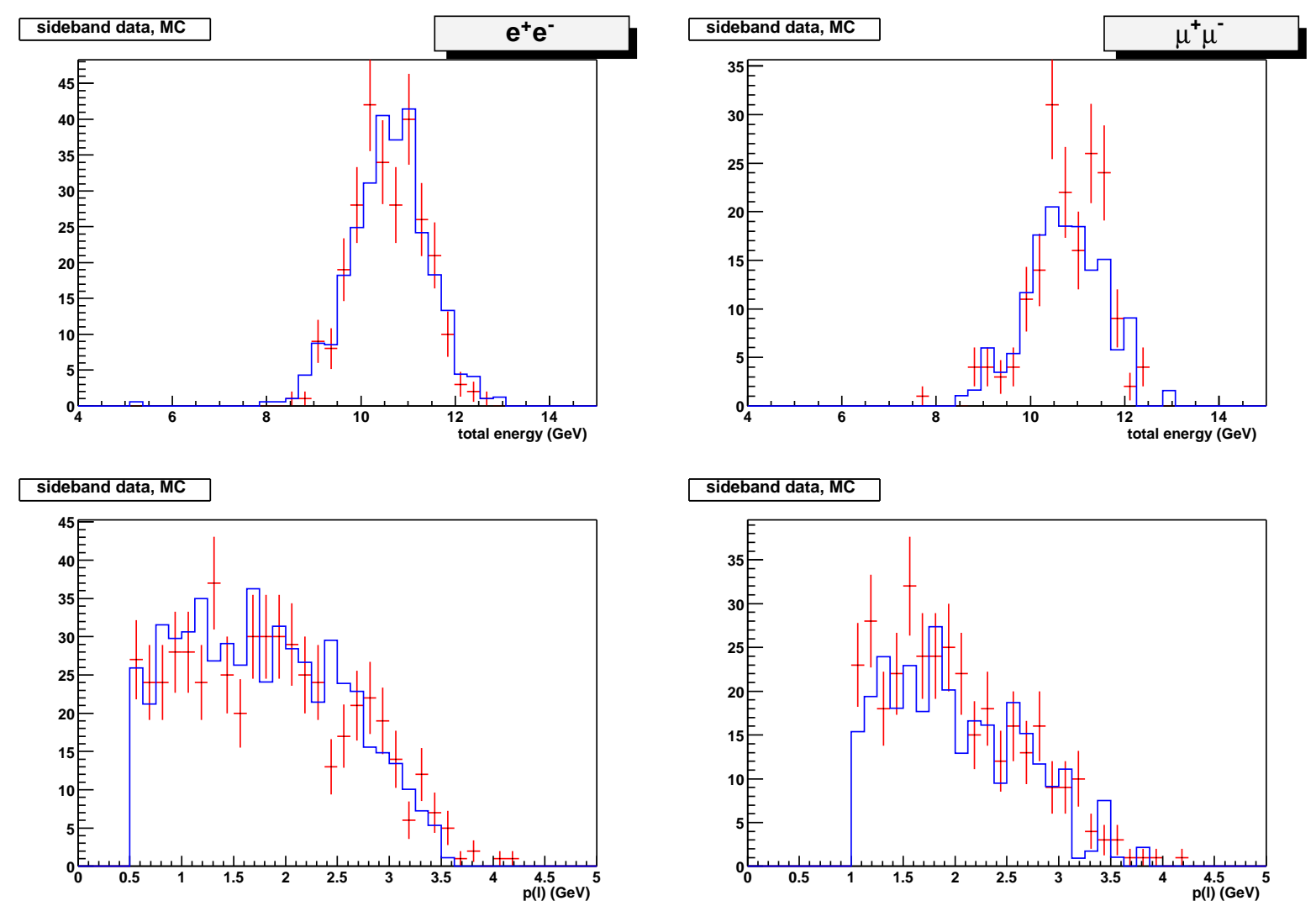

sideband data, MC
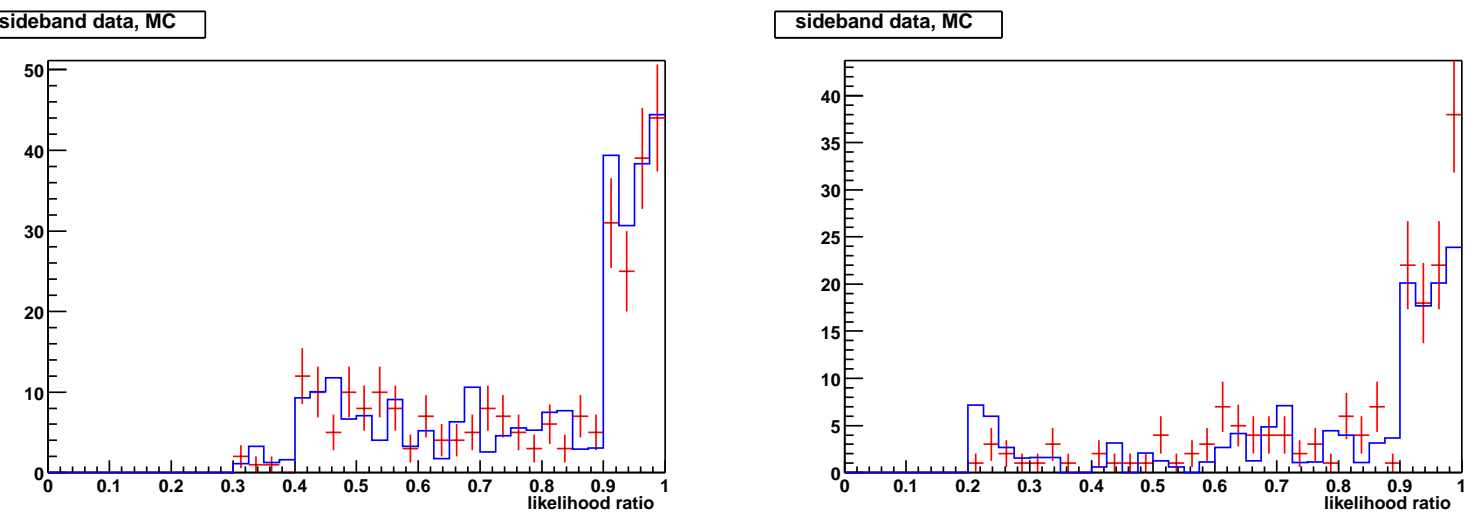

Figure 63: Distributions of total event energy, lepton momentum (both in the lab frame) and likelihood ratio for $B \rightarrow X_{s} \ell^{+} \ell^{-}$candidates in the sideband data sample for electrons (left) and muons (right). The points represent the data and the histograms the MC, scaled to correspond to an integrated luminosity of $81.9 \mathrm{fb}^{-1}$. 

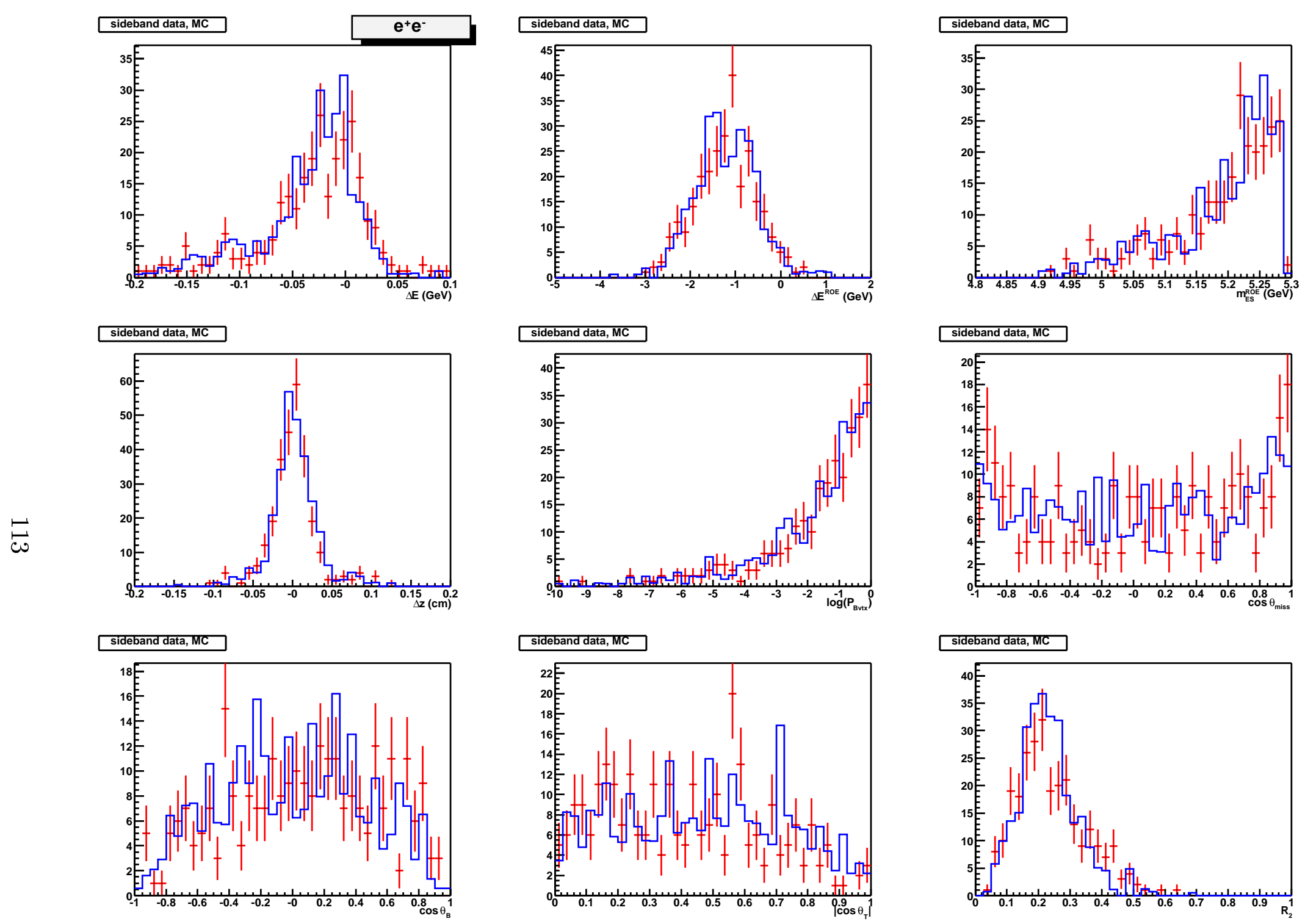

Figure 6.3: Distributions of the background-suppression variables entering the likelihood ratio for $B \rightarrow X_{s} e^{+} e^{-}$candidates in the sideband data sample. The points represent the data and the histograms the MC, scaled to correspond to an integrated luminosity of $81.9 \mathrm{fb}^{-1}$. 

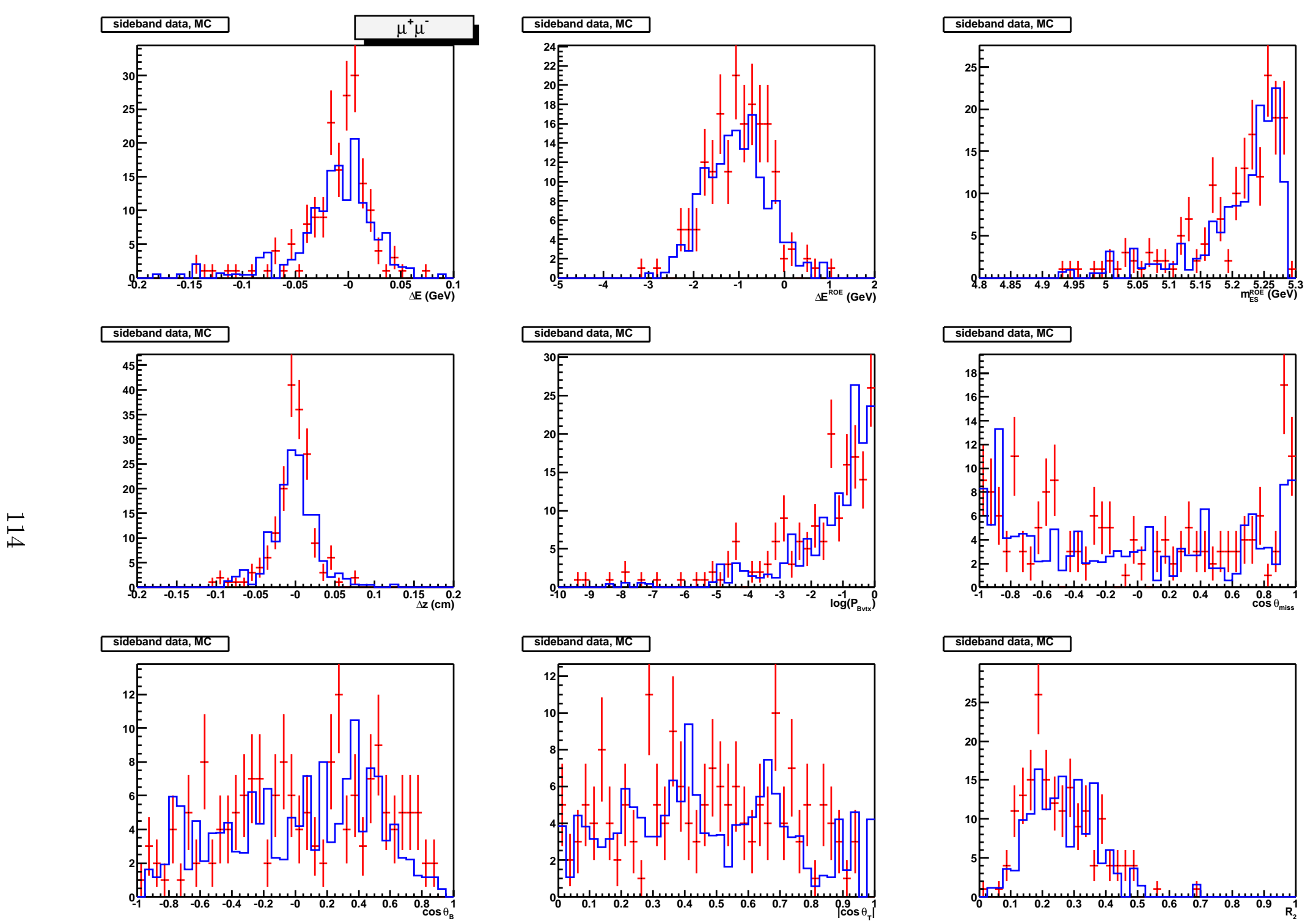

Figure 6.4: Distributions of the background-suppression variabes entering the likelihood ratio for $B \rightarrow X_{s} \mu^{+} \mu^{-}$candidates in the sideband data sample. The points represent the data and the histograms the MC, scaled to correspond to an integrated luminosity of $81.9 \mathrm{fb}^{-1}$. 


\title{
C H A P T E R 7
}

\author{
Peaking backgrounds
}

The three different peaking background types and the methods we use to suppress them were described in Sec. 5.3. In this chapter we estimate the remaining peaking background components after all analysis cuts have been applied.

\subsection{Charmonium peaking backgrounds}

Peaking backgrounds from $B \rightarrow J / \psi X$ and $B \rightarrow \psi(2 S) X$ decays are efficiently suppressed by the dilepton mass cuts. However, a very small contribution remains. To estimate this contribution, we used MC generated $B \rightarrow J / \psi X$ and $B \rightarrow \psi(2 S) X$ samples. Table 14 shows the raw numbers of fully reconstructed $B \rightarrow J / \psi X$ and $B \rightarrow \psi(2 S) X$ decays, in the signal region $m_{\mathrm{ES}}>5.27 \mathrm{GeV}$, and the corresponding

numbers scaled to an integrated luminosity of $81.9 \mathrm{fb}^{-1}$, before applying a cut on the likelihood ratio. We found a number of fully reconstructed $B \rightarrow J / \psi X$ decays with $J / \psi \rightarrow \mu^{+} \mu^{-}$in which one charged pion from the hadronic system was misidentified as a muon and one of the muons from the $J / \psi$ decay was not identified and therefore considered to be a pion. We refer to this situation as a case of $\mu \leftrightarrow \pi$ swap, and include these events in the total charmonium peaking background. These types of decays are likely to evade the charmonium veto cuts 
Table 14: Number of charmonium peaking background events from Monte Carlo simulation, before applying a cut on the likelihood ratio. The entries are scaled to an integrated luminosity of $81.9 \mathrm{fb}^{-1}$. The numbers given in parentheses are the raw event counts.

\begin{tabular}{lllll}
\hline \hline Mode & Generic $B \bar{B}$ MC & $B \rightarrow J / \psi X$ MC & $B \rightarrow \psi(2 S) X$ MC & Sum \\
\hline$J / \psi \rightarrow e^{-} e^{-}$ & $0.57(1)$ & $0.00(0)$ & - & $0.57(1)$ \\
$J / \psi \rightarrow \mu^{+} \mu^{-}$ & $0.00(0)$ & $0.00(0)$ & - & $0.00(0)$ \\
$\psi(2 S) \rightarrow e^{-} e^{-}$ & $0.40(1)$ & - & $0.21(1)$ & $0.61(2)$ \\
$\psi(2 S) \rightarrow \mu^{+} \mu^{-}$ & $0.40(1)$ & - & $0.21(1)$ & $0.61(2)$ \\
\hline$J / \psi \rightarrow \mu^{+} \mu^{-}$ & $2.28(4)$ & $1.20(2)$ & - & $3.48(6)$ \\
$(\mu \leftrightarrow \pi)$ swap & & & & \\
$\psi(2 S) \rightarrow \mu^{+} \mu^{-}$ & $0.00(0)$ & - & $0.00(0)$ & $0.00(0)$ \\
$(\mu \leftrightarrow \pi)$ swap & & & & \\
\hline
\end{tabular}

since the veto is applied on the wrong pair of particles (the reconstructed dilepton consists of one of the $J / \psi$ daughters and a pion from the hadronic system). In a future version of this analysis, one might consider vetoing against such an occurence, but no such requirement is imposed in the present analysis.

After scaling the raw number of peaking background decays to correspond to an integrated luminosity of $81.9 \mathrm{fb}^{-1}$, we find 0.59 and 2.05 events in the electron and muon channels, respectively. (The numbers in the right-hand column of Table 14 need to be divided by two.) Finally, we scale these numbers by a factor of 0.68 to account for the average efficiency for charmonium decays to pass the likelihood ratio cuts, as measured in the charmonium veto data. The final estimate for the number of charmonium peaking background events is thus $0.40 \pm 0.23$ in the electron channel and $1.39 \pm 0.49$ in the muon channel.

For the measurement of partial branching fractions in several bins of dilepton and hadronic mass, we need to estimate the fraction of background in each bin. For the distribution in terms of dilepton mass, we use the dilepton mass distribution of the small number of peaking background events found in the simulation. For 
Table 15: Number of charmonium peaking background events from Monte Carlo simulation for electron and muon modes combined in various bins of dilepton and hadronic mass, scaled to an integrated luminosity of $81.9 \mathrm{fb}^{-1}$.

\begin{tabular}{cccc}
\hline \hline $\begin{array}{c}m_{l l} \\
(\mathrm{GeV})\end{array}$ & \# bkg decays & $\begin{array}{c}m_{X s} \\
(\mathrm{GeV})\end{array}$ & \# bkg decays \\
\hline \hline $0.2-1.0$ & $0.00 \pm 0.00$ & $0.4-0.6$ & $0.69 \pm 0.21$ \\
$1.0-2.0$ & $0.19 \pm 0.19$ & $0.6-0.8$ & $0.05 \pm 0.02$ \\
$2.0-m_{J / \psi}$ & $1.18 \pm 0.48$ & $0.8-1.0$ & $0.37 \pm 0.11$ \\
$m_{J / \psi}-m_{\psi^{\prime}}$ & $0.41 \pm 0.21$ & $1.0-1.8$ & $0.67 \pm 0.20$ \\
$m_{\psi^{\prime}}-5.0$ & $0.00 \pm 0.00$ & & \\
\hline $1.0-2.45$ & $0.98 \pm 0.44$ & & \\
$3.8-5.0$ & $0.00 \pm 0.00$ & & \\
\hline
\end{tabular}

the distribution in terms of hadronic mass, we use the distribution found in the charmonium-veto data sample. The estimated numbers of background events are given in Table 15.

\subsection{Hadronic peaking backgrounds}

Another peaking background due to particle misidentification arises from hadronic $B$ meson decays where two hadrons of opposite charge have been misidentified as leptons. Since the $\pi \rightarrow e$ misidentification rate is negligible, this background is only considered in the muon channel. For example, the decay $B^{+} \rightarrow \bar{D}^{0} \pi^{+}$with $\bar{D}^{0} \rightarrow K^{+} \pi^{-}$is a background to $B^{+} \rightarrow K^{+} \mu^{+} \mu^{-}$when both pions are misidentified as muons.

These backgrounds are estimated using a sample of hadronic $B$ decays in BaBar data. The sample, later on referred to as $X_{s} h h$, is collected by imposing requirements identical to those for signal candidates, except that no lepton identification 
is applied. This results in a large sample of predominantly hadronic $B$ candidates. Each event is given a weight

$w=P\left(\pi^{+} \rightarrow \mu^{+}\right) P\left(\pi^{-} \rightarrow \mu^{-}\right)$, where $P\left(\pi^{+(-)} \rightarrow \mu^{+(-)}\right)$is the probability for positive (negative) pions to be misidentified by the positive (negative) muon signal selector.

The probabilities above are obtained from standard PID tables, which contain separate rates for each charge and each run condition block, as well as for varying degrees of binning in momentum and polar angle.

After applying the nominal likelihood ratio cuts, a weighted distribution in $m_{E S}$ is obtained, and a one-dimensional binned $\chi^{2}$ fit for signal plus combinatorial background gives the number of hadronic peaking background events.

The fits for each mode are shown in Figures 64, 65, and 66, and the signal yield for each fit is shown in Table 16. The hadronic peaking background for the muon mode is estimated to be $2.39 \pm 0.75$. The uncertainty is conservatively estimated by increasing all misidentification rates provided in the PID tables by $\pm 1 \sigma$, i.e. assuming the rates in the different bins of angle and momentum are fully correlated.

For the measurement of partial branching fractions in several bins of dilepton and hadronic mass, we need to estimate the fraction of background in each bin. The above procedure is repeated in each of the bins and the results for the $X_{s} h^{+} h^{-}$ topology are summarized in Table 17. 


\begin{tabular}{ll} 
Mode & $X_{s} h^{+} h^{-}$ \\
\hline$X_{s} \mu^{+} \mu^{-}$ & $2.39 \pm 0.75$ \\
\hline$K^{ \pm} \mu^{+} \mu^{-}$ & $1.04 \pm 0.38$ \\
$K^{ \pm} \pi^{0} \mu^{+} \mu^{-}$ & $0.24 \pm 0.06$ \\
$K^{ \pm} \pi^{ \pm} \mu^{+} \mu^{-}$ & $0.43 \pm 0.13$ \\
$K^{ \pm} \pi^{ \pm} \pi^{0} \mu^{+} \mu^{-}$ & $0.07 \pm 0.01$ \\
$K^{ \pm} \pi^{ \pm} \pi^{ \pm} \mu^{+} \mu^{-}$ & $0.15 \pm 0.04$ \\
$K_{s}^{0} \mu^{+} \mu^{-}$ & $0.19 \pm 0.06$ \\
$K_{s}^{0} \pi^{0} \mu^{+} \mu^{-}$ & $0.02 \pm 0.01$ \\
$K_{s}^{0} \pi^{ \pm} \mu^{+} \mu^{-}$ & $0.13 \pm 0.04$ \\
$K_{s}^{0} \pi^{ \pm} \pi^{0} \mu^{+} \mu^{-}$ & $0.04 \pm 0.01$ \\
$K_{s}^{0} \pi^{ \pm} \pi^{ \pm} \mu^{+} \mu^{-}$ & $0.02 \pm 0.01$
\end{tabular}

Table 16: Hadronic peaking backgrounds for $X_{s} \mu^{+} \mu^{-}$and its individual event topologies with the $X_{s} h^{+} h^{-}$sample.

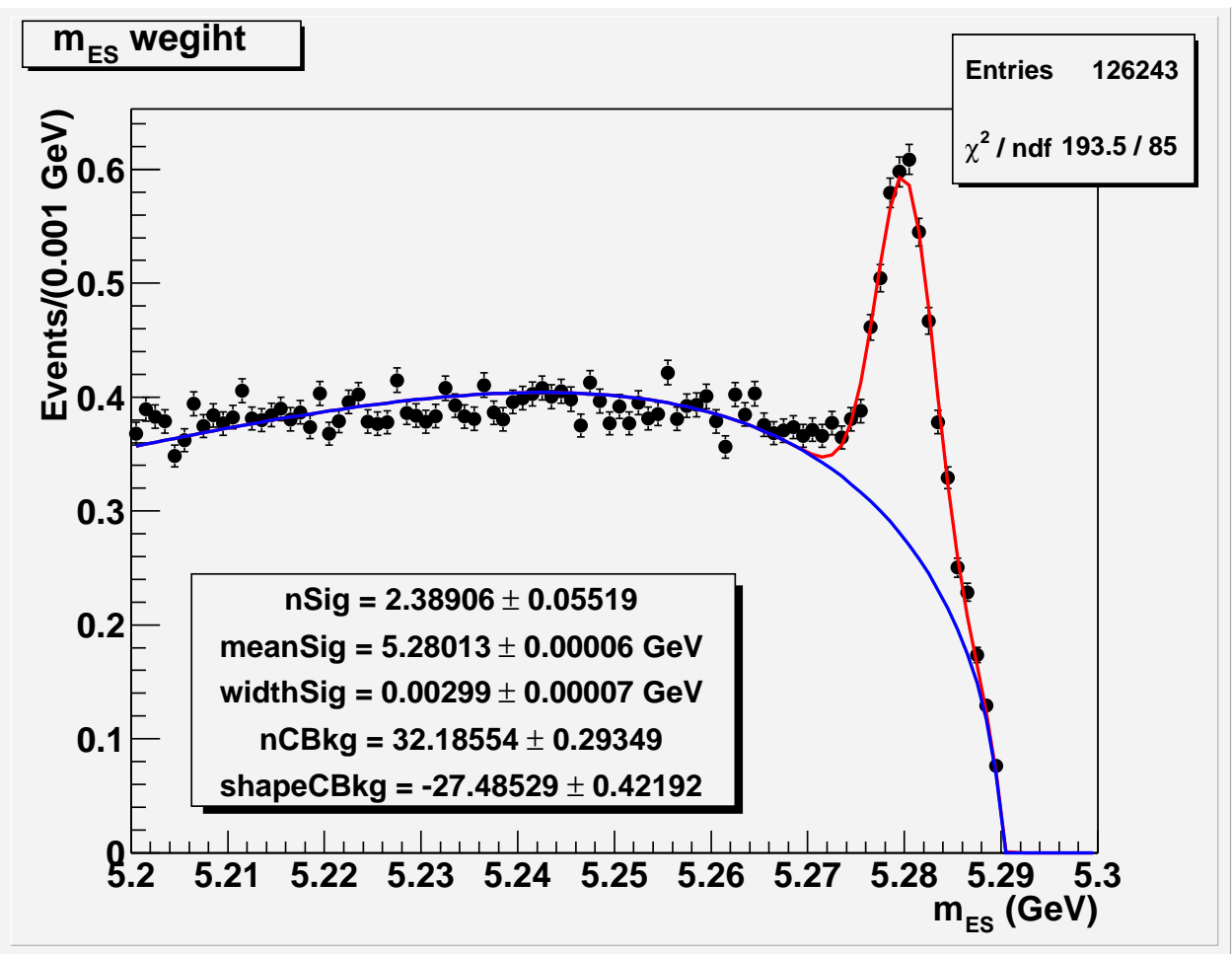

Figure 64: Weighted $m_{E S}$ distribution of $X_{s} h^{+} h^{-}$events. The red line is the total fit to a Gaussian signal plus an Argus background; the blue line is the Argus background portion of the fit. 

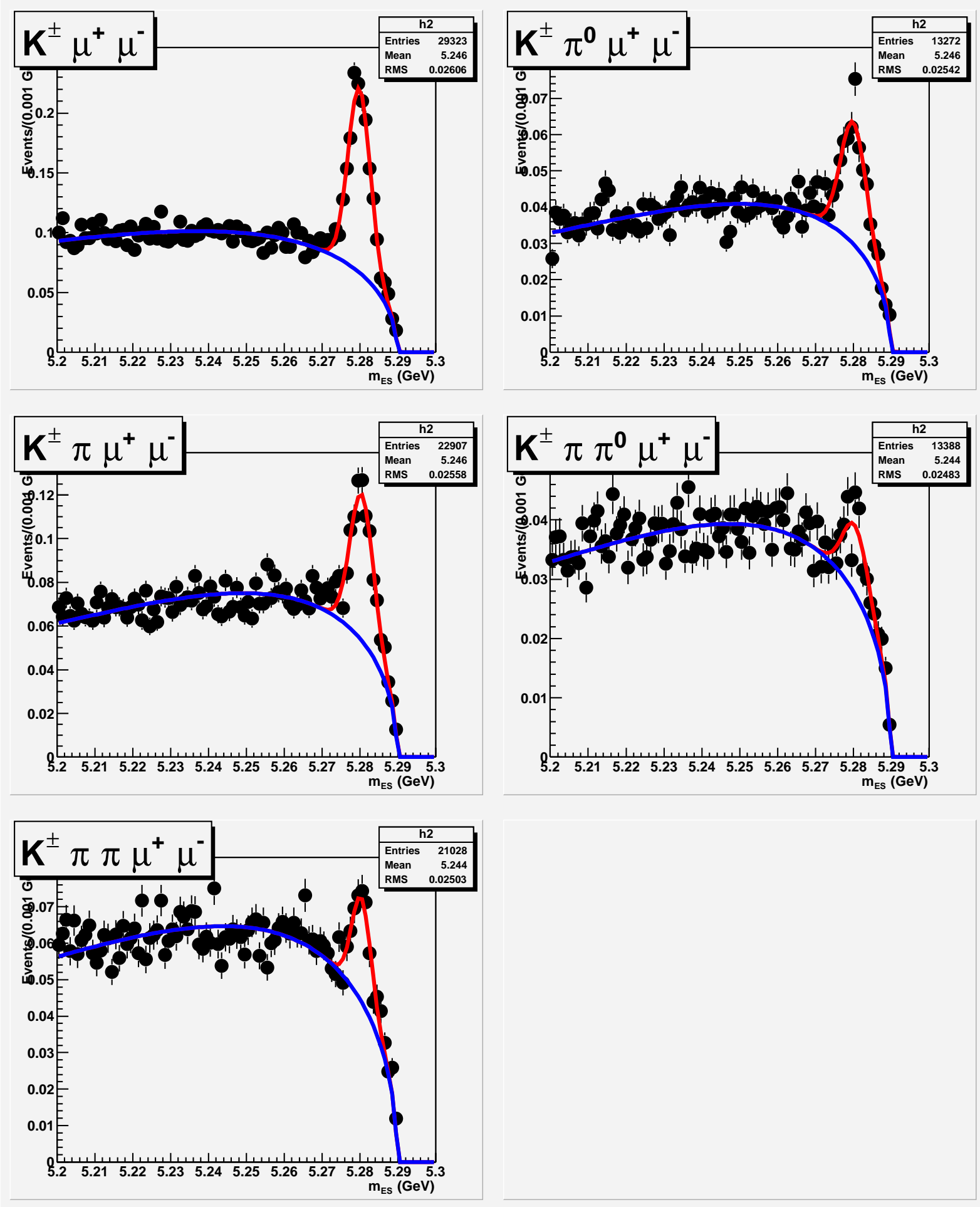

Figure 65: Weighted $m_{E S}$ distributions for $K^{ \pm} n \pi h^{+} h^{-}$final state topologies. The red line is the total fit to a Gaussian signal plus an Argus background; the blue line is the Argus background portion of the fit. 

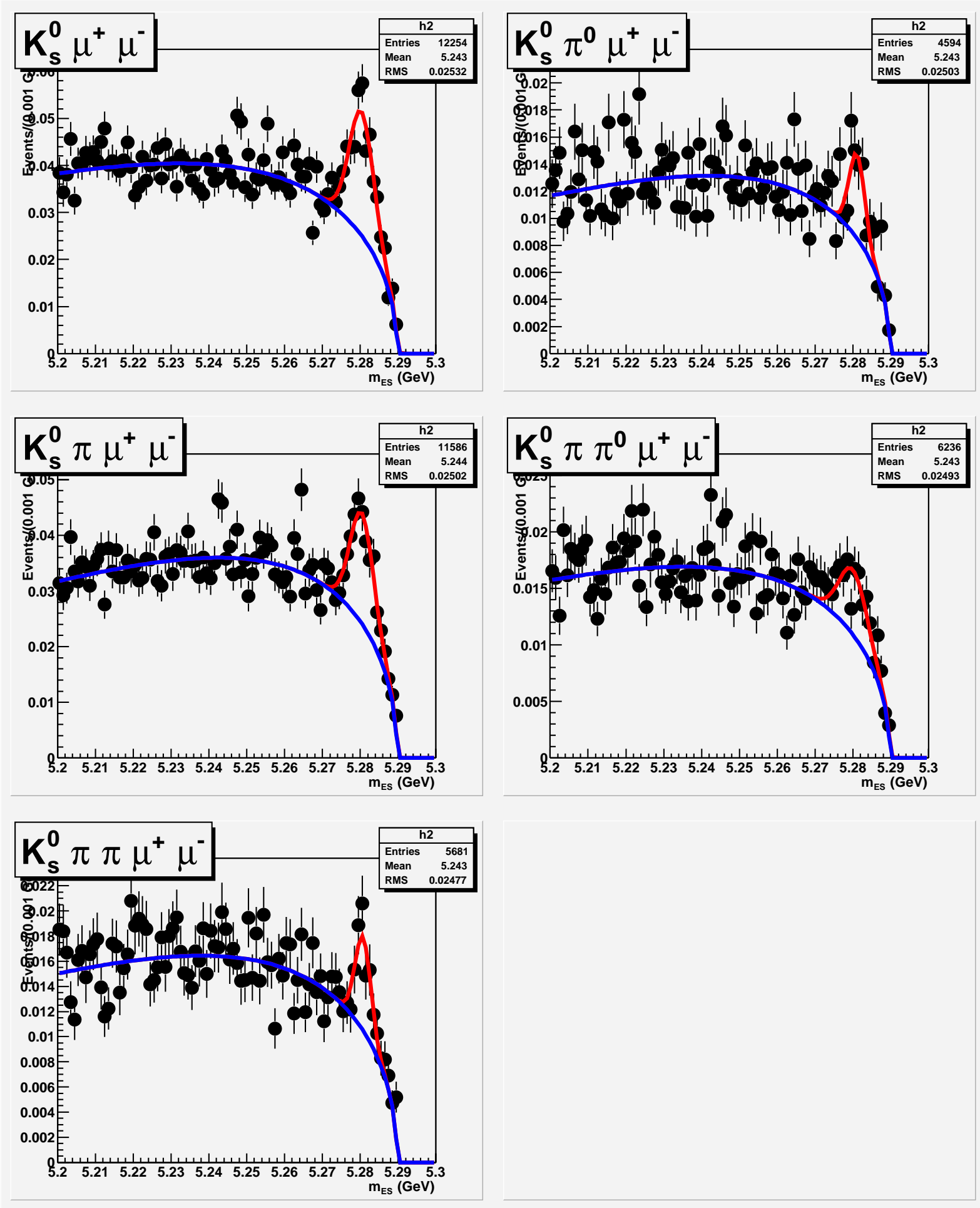

Figure 66: Weighted $m_{E S}$ distribution for $K_{s}^{0} n \pi h^{+} h^{-}$final state topologies. The red line is the total fit to a Gaussian signal plus an Argus background; the blue line is the Argus background portion of the fit. 
Table 17: Number of $X_{s} h^{+} h^{-}$hadronic peaking background events for muon modes in various bins of dilepton and hadronic mass.

\begin{tabular}{cccc}
\hline \hline $\begin{array}{c}m_{l l} \\
(\mathrm{GeV})\end{array}$ & \# bkg decays & $\begin{array}{c}m_{X s} \\
(\mathrm{GeV})\end{array}$ & \# bkg decays \\
\hline \hline $0.2-1.0$ & $0.06 \pm 0.03$ & $0.4-0.6$ & $1.24 \pm 0.45$ \\
$1.0-2.0$ & $0.11 \pm 0.03$ & $0.6-0.8$ & $0.22 \pm 0.06$ \\
$2.0-m_{J / \psi}$ & $0.29 \pm 0.08$ & $0.8-1.0$ & $0.37 \pm 0.11$ \\
$m_{J / \psi}-m_{\psi^{\prime}}$ & $0.58 \pm 0.16$ & $1.0-1.8$ & $0.55 \pm 0.15$ \\
$m_{\psi^{\prime}}-5.0$ & $1.32 \pm 0.47$ & & \\
\hline $1.0-2.45$ & $0.22 \pm 0.07$ & & \\
$3.8-5.0$ & $1.32 \pm 0.47$ & & \\
\hline
\end{tabular}




\section{H A P T E R 8}

\section{Maximum likelihood fit}

We extract the signal yield with an extended unbinned maximum likelihood fit to the $m_{E S}$ distribution in the region $m_{E S}>5.2 \mathrm{GeV}$. The likelihood function consists of 4 components: signal, charmonium peaking background, hadronic peaking background and continuum,

$$
\begin{aligned}
\mathcal{L}= & \frac{e^{-\left(N_{s i g}+N_{c \bar{c} s}+N_{h a d}+N_{c o m b}\right)}}{N !} \\
& \prod_{k=1}^{N}\left[\left(N_{s i g}+N_{c \bar{c} s}\right) \mathcal{P}^{s i g}\left(\mathrm{~m}_{\mathrm{ES}}^{\mathrm{k}}\right)+\mathrm{N}_{\mathrm{had}} \mathcal{P}^{\mathrm{had}}\left(\mathrm{m}_{\mathrm{ES}}^{\mathrm{k}}\right)+\mathrm{N}_{\text {comb }} \mathcal{P}^{\mathrm{comb}}\left(\mathrm{m}_{\mathrm{ES}}^{\mathrm{k}}\right)\right]
\end{aligned}
$$

The product runs over the total number $-N$ - of reconstructed candidate events. We perform separate fits for electron and muon modes, as well as a third fit where both samples are combined. The parameters $N_{s i g}, N_{c \bar{c} s}, N_{\text {had }}$ and $N_{\text {comb }}$ represent the signal yield, the charmonium peaking background, the hadronic peaking background and the combinatorial background, respectively, with corresponding PDFs given by $\mathcal{P}^{s i g}, \mathcal{P}^{\text {had }}$ and $\mathcal{P}^{\text {comb }}$. The fitted parameters are $N_{\text {sig }}, N_{\text {comb }}$ and the shape parameter of the PDF describing the combinatorial background $\mathcal{P}^{c o m b}$. The remaining parameters are fixed during the fit with values determined as follows: 
- Signal and charmonium peaking background shapes:

Both the signal and the charmonium peaking background shapes are described by one PDF, $\mathcal{P}^{s i g}$. For the functional form we use a Gaussian for the muon as well as the electron modes, since the Bremsstrahlung recovery leads to a negligible radiative tail in the $m_{E S}$ distribution of the latter. The shape parameters of the Gaussian are determined from Gaussian + Argus fits to the charmonium veto data sample as shown in Fig. 67. The fits yield signal peak positions at $m_{s i g}=5.280036 \pm 0.000051 \mathrm{GeV}, 5.280045 \pm 0.000070 \mathrm{GeV}$ and $5.280040 \pm 0.000041 \mathrm{GeV}$ in the electron, muon and electron+muon modes, respectively. The corresponding $m_{E S}$ resolutions are $\sigma_{s i g}=2.804 \pm 0.046 \mathrm{MeV}$ for electrons, $\sigma_{s i g}=2.613 \pm 0.061 \mathrm{MeV}$ for muons and $\sigma_{s i g}=2.745 \pm 0.037$ $\mathrm{MeV}$ for electrons and muons combined. The $m_{E S}$ distributions for truthmatched signal modes are illustrated in Fig. 68, and their Gaussian fit results are in good agreement with the shape parameters extracted from the fits to the charmonium veto data sample.

- Charmonium peaking background amount

The expected number of fully reconstructed $B \rightarrow J / \psi X$ and $B \rightarrow \psi(2 S) X$ decays $-N_{c \bar{c} s}-$ passing through the charmonium veto is estimated to be 0.40 and 1.39 events in the electron and muon channels, respectively, as determined from the MC, see Sec. 7.1.

- Hadronic peaking background

The amount of this background, $N_{\text {had }}=2.39$, is derived directly from data by performing the analysis without the lepton identification requirements, as described in Sec. 7.2. Parameters for the PDF $\mathcal{P}^{\text {had }}$ are also taken from that fit: the Gaussian mean is $m_{\text {had }}=5.28013 \pm 0.00006 \mathrm{GeV}$ and the Gaussian 

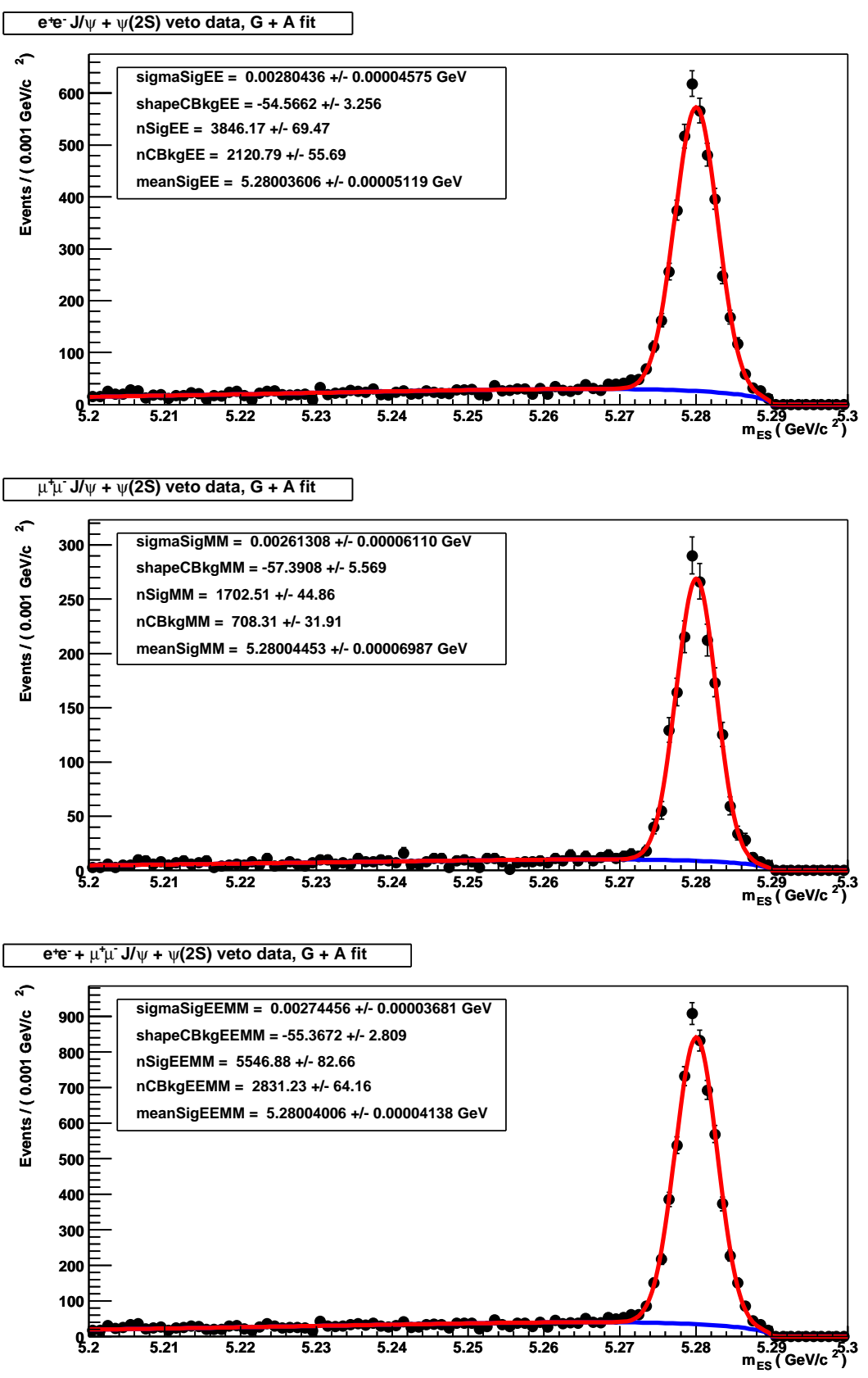

Figure 67: Distributions of $m_{E S}$ for $B \rightarrow X_{s} \ell^{+} \ell^{-}$candidates in the charmonium veto data sample for electrons (top), muons (middle), and both combined (bottom) after all cuts (but the inverted charmonium veto) are applied. 

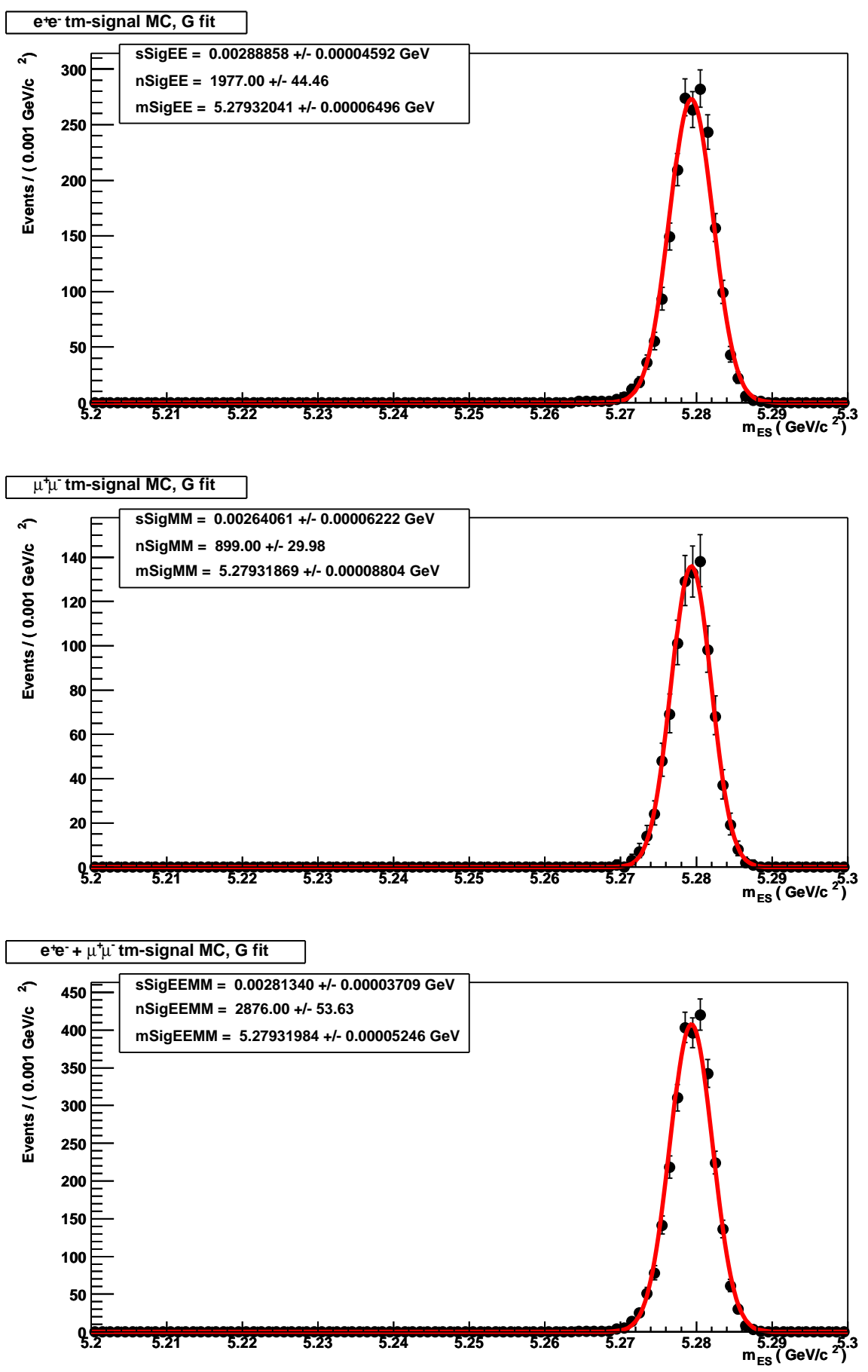

Figure 68: Distributions of $m_{E S}$ for truth-matched $B \rightarrow X_{s} \ell^{+} \ell^{-}$candidates for electrons (top) and muons (bottom). 
width is $\sigma_{\text {had }}=2.99 \pm 0.07 \mathrm{MeV}$, see Fig. 64 .

- Combinatorial background cutoff

The combinatorial background PDF, $\mathcal{P}_{k}^{c o m b}$, is given by an Argus shape which describes the combinatorial contribution from continuum events and $B \bar{B}$ events. The Argus cutoff is determined by the beam energy in the $\Upsilon(4 S)$ rest frame, $E_{\text {beam }}=5.290 \mathrm{GeV}$.

The signal yield obtained with the outlined procedure contains a small contribution from $B \rightarrow X_{s} \ell^{+} \ell^{-}$cross-feed events which is estimated from Monte Carlo, as will be shown in Section 9.1. The overall signal efficiency needed to compute branching fractions includes this small contribution. 


\section{H A P T E R 9}

\section{Results}

In this chapter we present the results from the measurements of the total branching fraction, the branching fractions in dilepton and hadronic mass bins and a measurement of the CP asymmetry.

\subsection{Total branching fractions}

The final $m_{\mathrm{ES}}$ histograms fitted as described in Chapter 8 are shown in Fig. 69. We measure a branching fraction of $\mathcal{B}\left(B \rightarrow X_{s} \ell^{+} \ell^{-}\right)=(5.64 \pm 1.46) \times 10^{-6}$ with statistical significance of $4.3 \sigma$, see Table 18. The significance is computed according to Signif $=\sqrt{2\left(\log \mathcal{L}_{\text {max }}-\log \mathcal{L}_{\text {max }}^{0}\right)}$, where $\mathcal{L}_{\text {max }}$ is the maximum likelihood for the nominal fit (with the signal yield as in Table 18) and $\mathcal{L}_{\text {max }}^{0}$ is a maximum likelihood obtained with a different fit for which the signal yield is fixed at $N_{\text {sig }}=0$.

The branching fraction $\mathcal{B}$ for the signal is calculated with

$$
\mathcal{B}=\frac{N_{\text {sig }}}{2 N_{B \bar{B}} \epsilon},
$$

where $N_{B \bar{B}}=(88.9 \pm 1.0) \times 10^{6}$ is the number of $B \bar{B}$ pairs produced in 81.9 $\mathrm{fb}^{-1}$ and $\epsilon$ is a signal efficiency calculated from the total truth-matched signal 

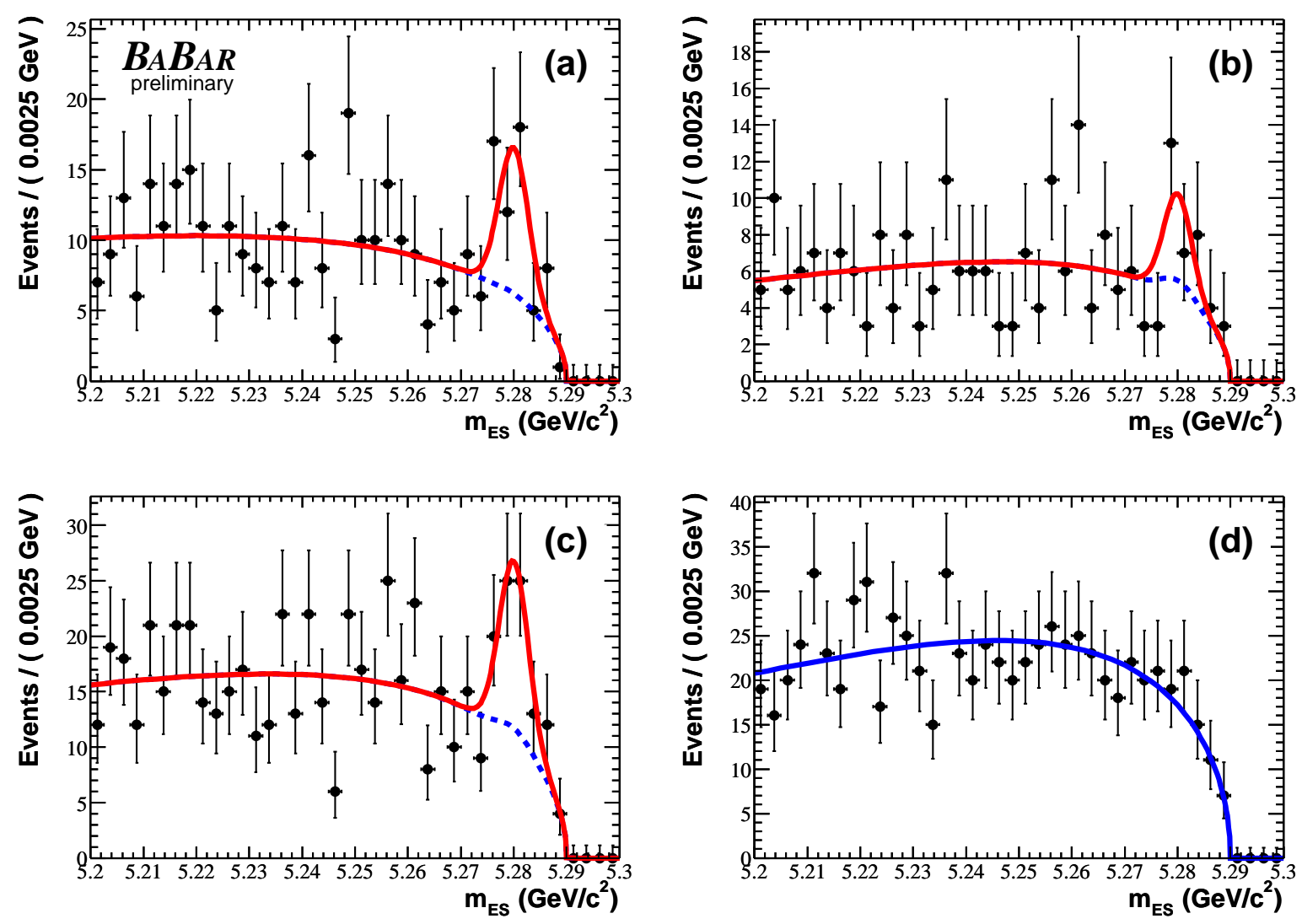

Figure 69: Distributions of $m_{E S}$ for data in the (a) $e^{+} e^{-}$, (b) $\mu^{+} \mu^{-}$, (c) $e^{+} e^{-}$ $+\mu^{+} \mu^{-}$, and (d) $e^{ \pm} \mu^{\mp}$ channels. The red line is the result of the fit, with the total background component shown in the dashed (or solid for $e^{ \pm} \mu^{\mp}$ ) blue line.

Table 18: Results of the fit to data: signal yield, peaking background (fixed in the fit), combinatorial background, signal significance, signal efficiency and branching fraction.

\begin{tabular}{lccccccc} 
Sample & $N_{\text {sig }}$ & $N_{\text {čss }}$ & $N_{\text {peak }}$ & $N_{\text {comb }}$ & Signif. & $\epsilon$ & $\mathcal{B}\left(\times 10^{-6}\right)$ \\
\hline$X_{s} e^{+} e^{-}$ & $29.22 \pm 8.28$ & 0.40 & 0.0 & $319.4 \pm 18.9$ & 4.0 & $2.74 \%$ & $6.00 \pm 1.70$ \\
$X_{s} \mu^{+} \mu^{-}$ & $11.25 \pm 6.19$ & 1.39 & 2.39 & $207.0 \pm 15.2$ & 2.0 & $1.26 \%$ & $5.04 \pm 2.77$ \\
$X_{s} \ell^{+} \ell^{-}$ & $40.06 \pm 10.35$ & 1.79 & 2.39 & $526.8 \pm 24.3$ & 4.3 & $2.00 \%$ & $5.64 \pm 1.46$ \\
\hline
\end{tabular}


reconstruction efficiency, multiplied by a factor to account for the small cross-feed component picked up by the signal Gaussian in the fit process.

The truth-matched signal reconstruction efficiency is shown in the last column of Tables 28 and 29, and is calculated as the ratio between the number of fully reconstructed signal decays in the ten considered modes and the total number of generated signal events in all topologies. The additional cross-feed factor is determined by comparing the fit results on the data obtained with the likelihood function from Chapter 8, see Fig. 69, to those obtained with a modified likelihood function that includes an extra PDF for the cross-feed contribution, the green line in Fig. 70. The shape of the cross-feed PDF is determined from MC, see Fig. 71 and is fixed during the maximum likelihood fit, the relative cross-feed yield, with respect to the signal yield, is derived from simulation and fixed to 0.47 and 0.37 for electron and muon modes, respectively. The additional signal yield obtained in the nominal fit due to cross-feed is determined to be $1.67,0.56$, and 2.10 events in the electron, muon, and electron+muon channels, respectively. This corresponds to a relative increase of $6.1 \%, 5.3 \%$, and $5.5 \%$ in the signal efficiency with respect to the efficiency obtained with truth-matched decays only. The signal efficiency calculated for truth-matched signal decays is therefore increased by a factor of 1.055, as determined from the larger statistics electron+muon channel. The overall signal efficiencies are thus estimated to be $2.74 \%, 1.26 \%$, and $2.00 \%$ in the electron, muon, and electron+muon channels, respectively. The efficiency for the electron+muon channel is computed as a simple average of the electron and muon channel efficiencies, since the branching fractions are expected to be equal after the requirement $m_{l l}>0.2 \mathrm{GeV}$. Branching fractions are given in Table 18 . For the combined electron and muon channels, the efficiency and branching fraction are to be understood as averages over the electron and muon channels. 

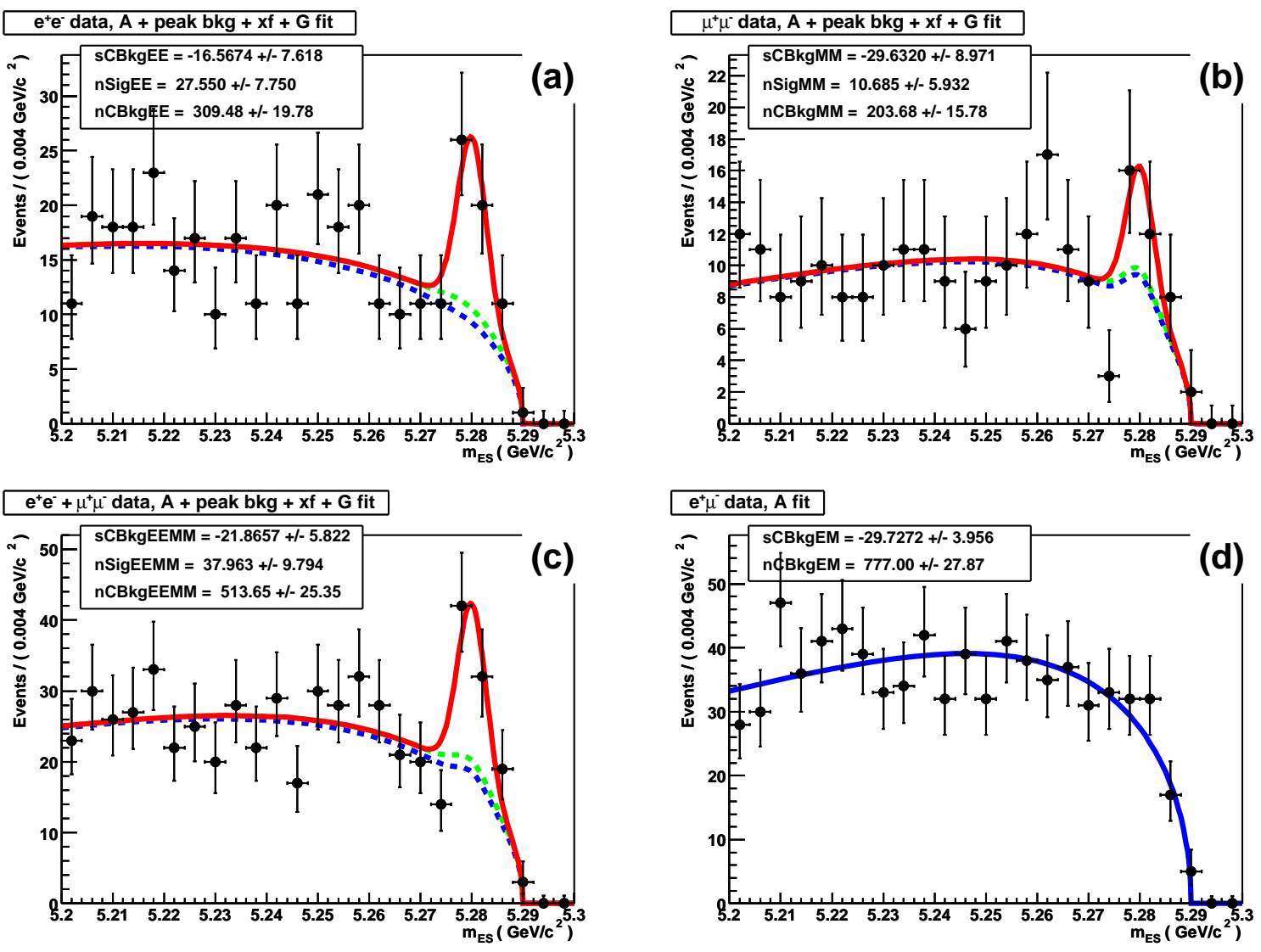

Figure 70: Distributions of $m_{E S}$ for data in the (a) $e^{+} e^{-}$, (b) $\mu^{+} \mu^{-}$, (c) $e^{+} e^{-}$ $+\mu^{+} \mu^{-}$, and (d) $e^{ \pm} \mu^{\mp}$ channels. The red line is the result of the fit, with the total background component shown in the dashed blue line and the sum of total background + signal cross-feed contribution in the dashed green line; a solid blue line is used for the total background in the $e^{ \pm} \mu^{\mp}$ channel. 

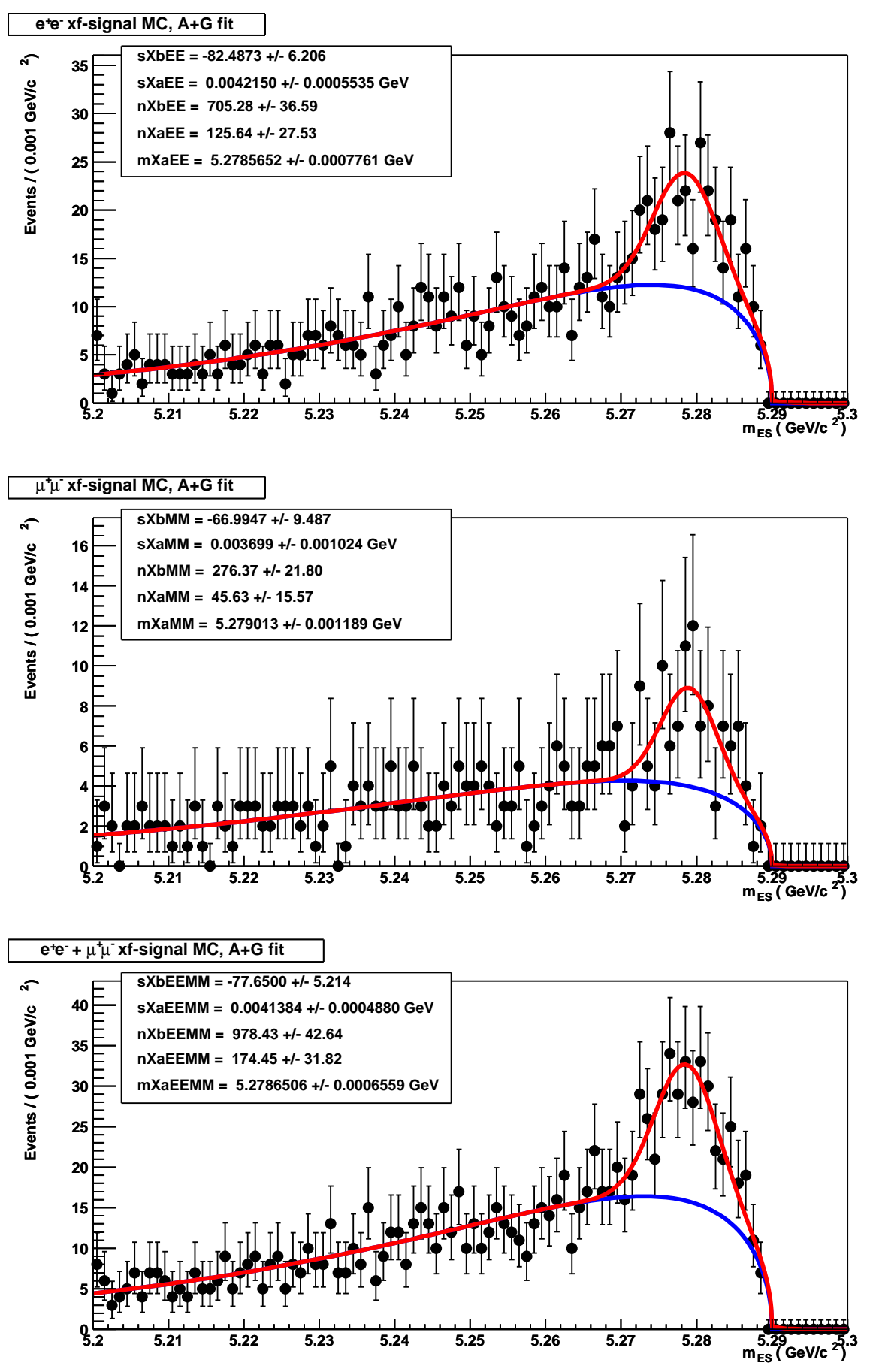

Figure 71: Distributions of $m_{E S}$ for $B \rightarrow X_{s} \ell^{+} \ell^{-}$candidates in the signal MC that fail truth-matching, i.e. cross-feed events for electrons (top) and muons (bottom). A Gaussian + Argus is used to parametrize the cross-feed PDF for both dilepton modes. 
Table 19: Signal yields for electron and muon modes combined in various bins of dilepton and hadronic mass.

\begin{tabular}{cccr}
\hline \hline $\begin{array}{c}m_{l l} \\
\left(\mathrm{GeV} / c^{2}\right)\end{array}$ & $N_{\text {sig }}$ & $\begin{array}{c}m_{X s} \\
\left(\mathrm{GeV} / c^{2}\right)\end{array}$ & \multicolumn{1}{c}{$N_{\text {sig }}$} \\
\hline \hline $0.2-1.0$ & $0.4 \pm 2.0$ & $0.4-0.6$ & $16.3 \pm 5.3$ \\
$1.0-2.0$ & $12.2 \pm 4.8$ & $0.6-0.8$ & $3.5 \pm 2.6$ \\
$2.0-m_{J / \psi}$ & $12.6 \pm 6.0$ & $0.8-1.0$ & $6.9 \pm 4.3$ \\
$m_{J / \psi}-m_{\psi^{\prime}}$ & $5.8 \pm 5.0$ & $1.0-1.8$ & $12.3 \pm 7.3$ \\
$m_{\psi^{\prime}}-5.0$ & $8.6 \pm 4.3$ & & \\
\hline $1.0-2.45$ & $15.9 \pm 6.2$ & & \\
$3.8-5.0$ & $8.6 \pm 4.3$ & & \\
\hline
\end{tabular}

\subsection{Partial branching fractions in dilepton and hadronic mass bins}

Signal yields are also extracted for the electron and muon channels combined in a series of dilepton and hadronic mass ranges. The individual $m_{E S}$ distributions and respective fits are shown in Figs. 72 and 73. The nominal fit procedure is performed with the nominal signal Gaussian shape parameters and numbers of peaking background decays as described in Secs. 7.1 and 7.2. The signal yields are given in Table 19.

To compute the partial branching fraction in each of the bins, we need to estimate the additional efficiency due to cross-feed $B \rightarrow X_{s} \ell^{+} \ell^{-}$events. Overall, the number of signal events attributed to cross-feed events is estimated to be $5.5 \%$ of the number of fully reconstructed events (i.e. truth-matched decays). To distribute these events among $m_{X s}$ and $m_{l l}$ bins, we use the simulated $m_{X s}$ and $m_{l l}$ distributions for cross-feed $B \rightarrow X_{s} \ell^{+} \ell^{-}$events. This approach assumes that the $m_{E S}$ shape of the cross-feed events does not change as a function of $m_{X s}$ and $m_{l l}$. Table 20 summarizes the efficiency correction factors used to account for the cross-feed 

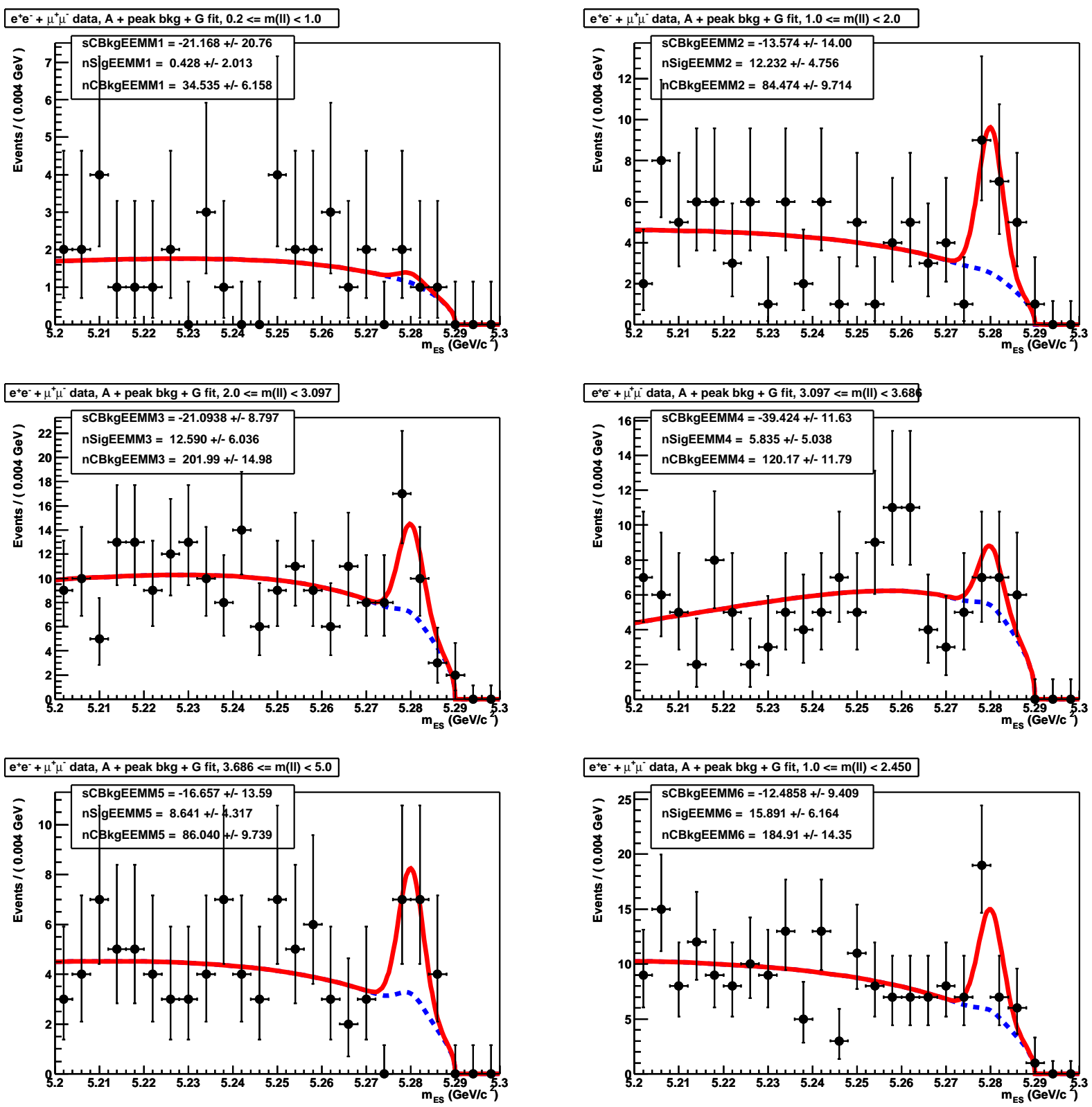

Figure 72: Distributions of $m_{E S}$ for $B \rightarrow X_{s} \ell^{+} \ell^{-}$candidates (electron+muon channels combined) in different ranges of dilepton mass (see range in the box above each plot). The red line is the result of the fit, with the total background component shown in the dashed blue line. 

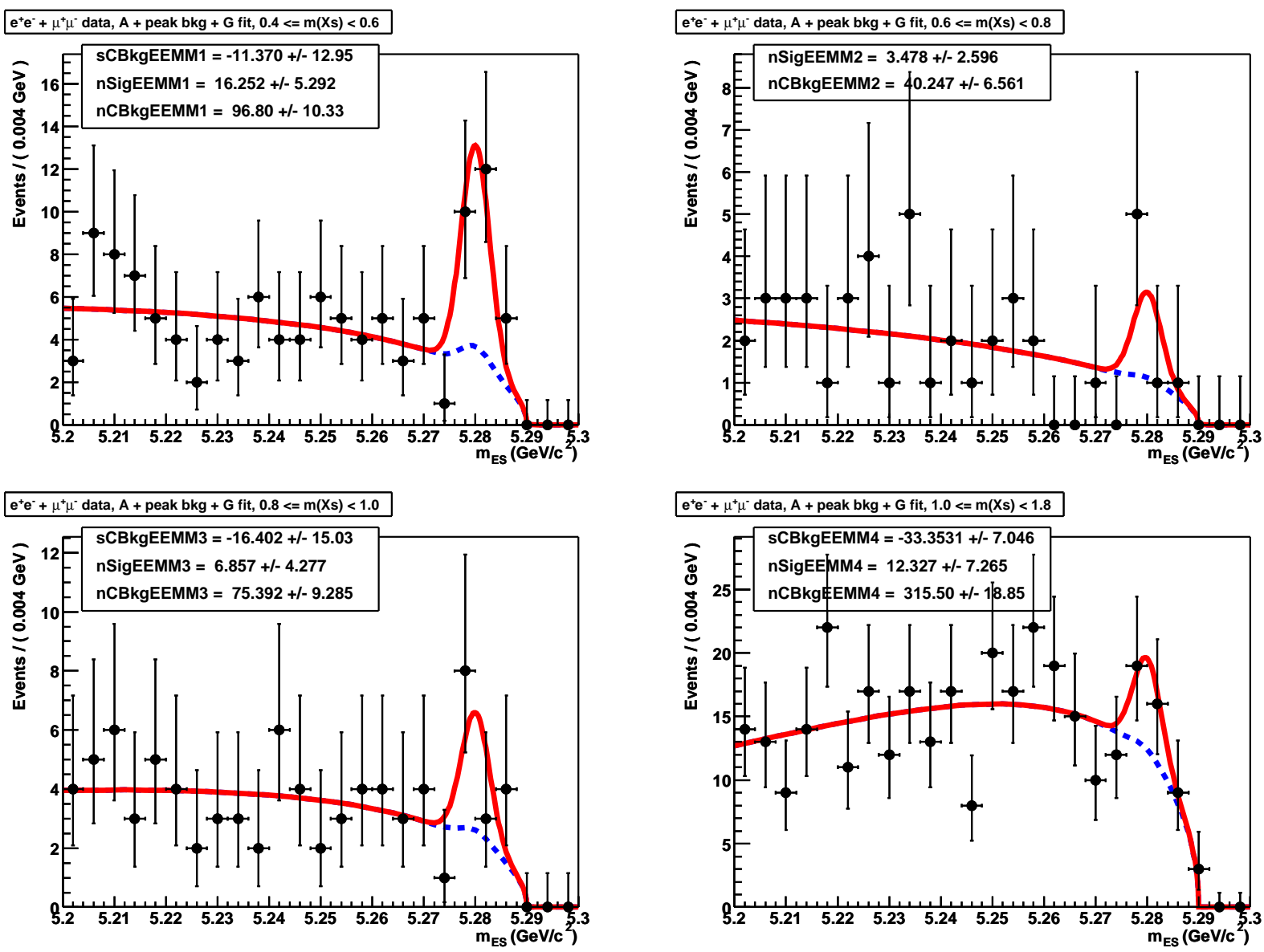

Figure 73: Distributions of $m_{E S}$ for $B \rightarrow X_{s} \ell^{+} \ell^{-}$candidates (electron+muon channels combined) in different ranges of hadronic mass (see range in the box above each plot). The red line is the result of the fit, with the total background component shown in the dashed blue line. 
Table 20: Efficiency (in percent) for truth-matched $B \rightarrow X_{s} \ell^{+} \ell^{-}$decays, enhancement factor to account for the cross-feed contribution to the signal yield, and final efficiency (in percent).

\begin{tabular}{lccc}
\hline \hline Sample & Raw efficiency & Factor & Efficiency \\
\hline \hline$e^{+} e^{-}$ & 2.60 & 1.06 & 2.74 \\
$\mu^{+} \mu^{-}$ & 1.19 & 1.06 & 1.26 \\
$\ell^{+} \ell^{-}$ & 1.89 & 1.06 & 2.00 \\
\hline$m\left(X_{s}\right)\left(\mathrm{GeV} / c^{2}\right)$ & & & \\
\hline $0.4-0.6$ & 8.49 & 1.02 & 8.66 \\
$0.6-0.8$ & 2.85 & 1.06 & 3.03 \\
$0.8-1.0$ & 2.76 & 1.09 & 2.99 \\
$1.0-1.8$ & 0.91 & 1.09 & 0.99 \\
\hline$m\left(\ell^{+} \ell^{-}\right)\left(\mathrm{GeV} / c^{2}\right)$ & & & \\
\hline $0.2-1.0$ & 1.07 & 1.45 & 1.55 \\
$1.0-2.0$ & 2.05 & 1.03 & 2.10 \\
$2.0-m_{J / \psi}$ & 1.94 & 1.04 & 2.02 \\
$m_{J / \psi}-m_{\psi^{\prime}}$ & 1.57 & 1.07 & 1.68 \\
$m_{\psi^{\prime}}-5.0$ & 3.49 & 1.10 & 3.82 \\
$1.0-2.45$ & 2.33 & 1.04 & 2.42 \\
$3.795-5.0$ & 4.45 & 1.10 & 4.88 \\
\hline \hline
\end{tabular}

contribution to the signal yield in the different $m_{X s}$ and $m_{l l}$ bins.

\subsection{CP asymmetry}

Using the fit described above, we can also extract the number of signal events in separate samples of $B$ and $\bar{B}$ decays, and thus measure the direct CP asymmetry in the $B \rightarrow X_{s} \ell^{+} \ell^{-}$decay process. To do so, we use only self-tagging decay modes, i.e., the 3 modes with hadronic systems consisting of $X_{s}=K_{s}, K_{s} \pi^{0}$, and $K_{s} \pi^{+} \pi^{-}$are removed. The $m_{E S}$ distributions for $\bar{B}$ and $B$ decays are shown in Fig. 74. As for the nominal fit, we extract the signal Gaussian shape parameters from the corresponding 7 modes in the charmonium veto sample. Furthermore, we 
assume identical shapes for the $B$ and $\bar{B}$ samples, as well as peaking backgrounds, i.e., we divide the amount of peaking background equally between the $B$ and $\bar{B}$ subsamples. Using our control samples, we found no evidence for these asumptions to be incorrect. Fit results are $N_{\text {sig }}^{\bar{B}}=14.7 \pm 6.5$ and $N_{\text {sig }}^{B}=22.9 \pm 7.4$, which corresponds to a charge asymmetry of $A_{C P} \equiv \frac{N_{s i g}^{\bar{B}}-N_{s i g}^{B}}{N_{s i g}^{\bar{B}}+N_{s i g}^{B}}=-0.22 \pm 0.26$.

This result is consistent with the expectation of no asymmetry in the Standard Model. Ali and Hiller [25] predict $A_{C P}=\left(0.19_{-0.19}^{+0.17}\right) \%$. 

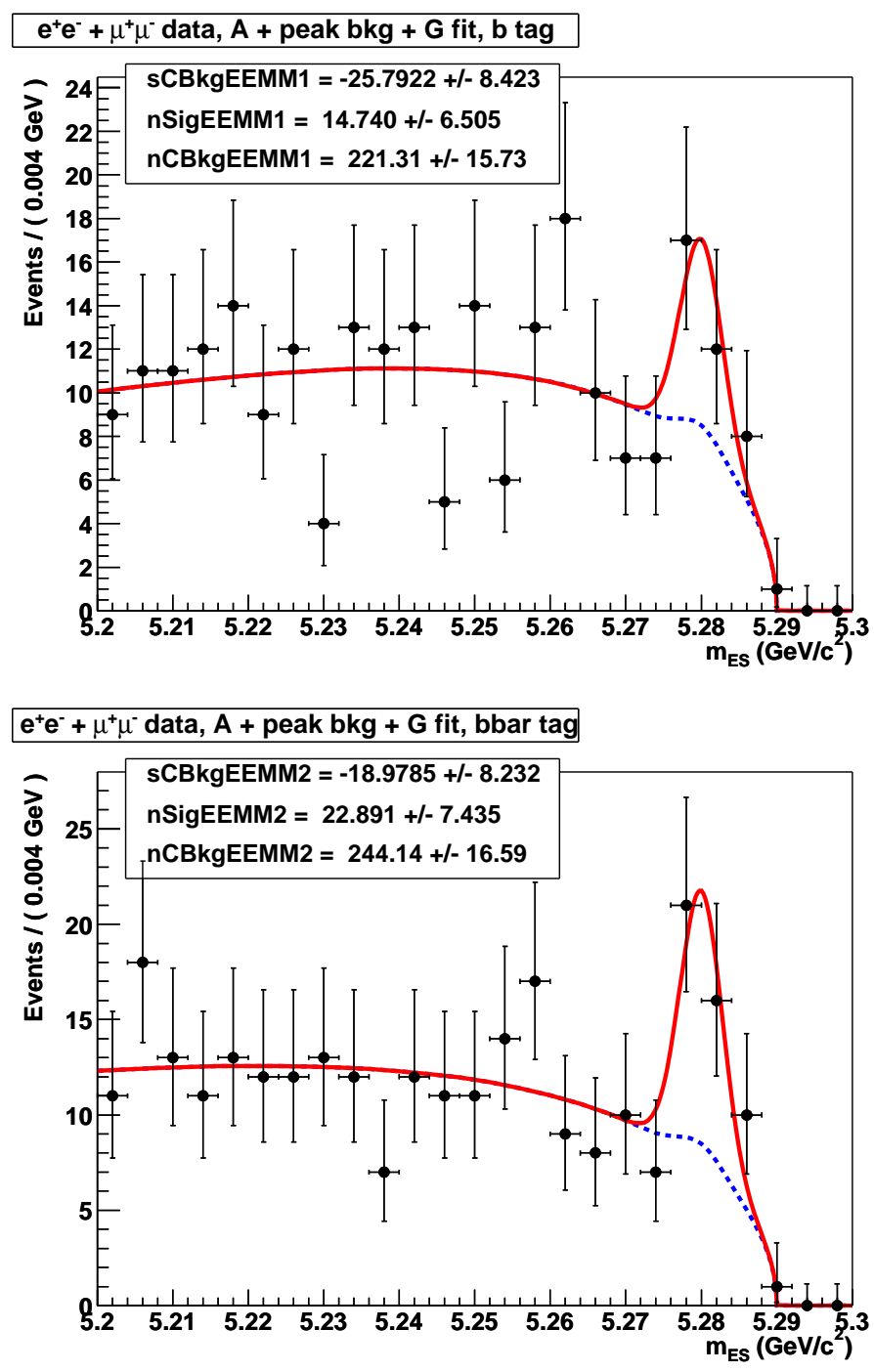

Figure 74: Distributions of $m_{E S}$ for combined electron and muon data in selftagged $\bar{B}$ (top) and $B$ (bottom) decays. The red line is the result of the fit, with the total background component shown in the dashed blue line. 


\title{
C H A P T E R 10
}

\author{
Systematic uncertainties
}

The systematic uncertainties are of two different types, those that affect the extraction of the number of signal decays and those that affect the calculation of the branching fraction.

\subsection{Signal yield systematics}

Uncertainties affecting the extraction of the signal yield are as follows:

- Signal Gaussian shape parameters Signal shape parameters are taken from a fit to the charmonium veto sample, see Fig. 67. The mean and width of the signal Gaussian are varied by $\pm 1 \sigma$, where $\sigma$ is the statistical uncertainty from the fit.

- Signal shape

Fits can be performed with different signal function shapes. Both Crystal Ball and Novosibirsk functions provide adequate description of the signal and allow for an asymmetric $m_{E S}$ distribution. The difference (from the nominal fit) in signal yields is taken as a systematic uncertainty. 
- Hadronic peaking background

The amount of hadronic peaking background $\left(B \rightarrow X_{s} h^{+} h^{-}\right)$is appreciable only in the muon channels and is estimated directly from data (see Table 16). The associated uncertainty is dominated by the uncertainty in the $\pi \rightarrow \mu$ misidentification probability. Gaussian shape parameters (mean and width) are also varied according to the fit result to the sample obtained without lepton identification.

- Charmonium peaking background

The other source of peaking background originates from $B \rightarrow J / \psi X$ and $B \rightarrow$ $\psi(2 S) X$ decays that evade the charmonium veto. The amount of background is estimated from Monte Carlo simulation. This amount is varied according to the error expected for the number of selected events in the simulation.

The total uncertainties in the signal yield are \pm 1.26 and \pm 0.90 events in the electron and muon channels, respectively. See Table 21 for a summary of the branching fraction systematic errors due to the signal yield uncertainties.

\subsection{Branching fraction systematics}

The uncertainties affecting the estimate of the signal selection efficiency (or the number of $B$ mesons in the sample) are as follows:

- Tracking efficiency

The systematic uncertainties of the charged track reconstruction efficiencies are determined by the Tracking Efficiency Task Force at BABAR. For the charged track reconstruction efficiency we apply a flat systematic uncertainty of $\pm 1.3 \%$ per track for lepton and kaon tracks, and $\pm 0.8 \%$ per track for the 
pion tracks. These values were chosen based on the PID selectors we use, and the average track multiplicity in our MC samples.

- $K_{S}^{0}$ efficiency

For $K_{S}^{0}$ the uncertainty is $1.5 \%$ per $K_{S}^{0}$ estimated using the official recipe [26].

- $\pi^{0}$ efficiency

For neutral pions, the uncertainty is $\pm 5.0 \%$ per $\pi^{0}$. This increases to $5.6 \%$ per $\pi^{0}$ after taking care of an additional uncertainty added in quadrature corresponding to whether one accounts for uncorrelated errors or not.

- Charged particle ID efficiency

The efficiency systematics for charged particle identification $(e, \mu$ and $K)$ can be estimated in two ways. First, we take uncertainties from the PID tables and properly average them using signal MC. This method yields uncertainties of $0.56 \%$ per electron, $3.85 \%$ per muon and $1.06 \%$ per kaon. Another approach has been taken for the study of exclusive $B \rightarrow K \ell^{+} \ell^{-}$and $B \rightarrow K^{*} \ell^{+} \ell^{-}$decays [27], where the efficiencies and associated uncertainties are computed from the charmonium veto sample. This second approach yields uncertainties of $0.65 \%$ per electron, $1.6 \%$ per muon and $1.0 \%$ per kaon. The latter approach is used for this analysis. In the case of the electron selection, we apply an additional uncertainty of $0.2 \%$ per electron (added linearly) to account for the difference in PID efficiency observed between electron candidates in the ChargedTracks selector (as used in this analysis) and the GoodTracksLoose selector (as used by the exclusive $B \rightarrow K^{(*)} \ell^{+} \ell^{-}$analysis) lists. The uncertainty is thus $0.85 \%$ per electron.

- Likelihood ratio efficiency 
The efficiency of the final step of background rejection is checked with the charmonium veto sample. After background subtraction, the efficiency of the likelihood ratio cuts is found to be $68.0 \%$ (68.1\%) in the electron (muon) data and $65.5 \%$ (65.9\%) in the electron (muon) MC. Given the available statistics the level of disagreement between data and $\mathrm{MC}$ is about $2 \sigma$. We take the discrepancy of $3.9 \%$ (3.3\%) in the electron (muon) channel to the systematic uncertainty in the likelihood ratio cut efficiency.

- Cross-feed efficiency

The fraction of cross-feed that ends up in the signal Gaussian is determined by comparing the fit results on the data obtained with either the nominal likelihood function or with a modified version that incorporates an extra PDF that represents the shape of the cross-feed contribution, as determined from MC. The additional signal yield obtained in the nominal fit is taken to correspond to an increase of $5.5 \%$ in the signal efficiency with respect to the efficiency obtained with truth-matched decays only. The number of cross-feed events included in the signal Gaussian are consistent with those obtained in toy MC studies. These studies indicate that the distribution in the number of cross-feed events has an R.M.S. close to the average number of cross-feed events. Therefore, we assign an uncertainty of $\pm 100 \%$ in the additional efficiency due to the signal cross-feed contribution.

- Fermi motion model

The parameters of the Fermi motion model are set to the values that best describe inclusive $b \rightarrow s \gamma$ decays measurements at CLEO [28]: $p_{F}=410 \mathrm{MeV} / c$ and $m_{q}=0$. Uncertainties in the parameter $p_{F}$ can be extracted using measurements of HQET parameters from measurements of hadronic moments in 
semileptonic decays: $\lambda_{1}=-0.24 \pm 0.11 \mathrm{GeV}^{2}[29]$ and from inclusive $b \rightarrow s \gamma$ decays: $\bar{\Lambda}=0.35 \pm 0.13 \mathrm{GeV}[28]$. These can be related to the parameters of the Fermi motion model, see Ref. [30], to yield $p_{F}=\sqrt{\frac{2}{3}\left(-\lambda_{1}\right)}=0.40_{-0.11}^{+0.08}$ $\mathrm{GeV}$ and $p_{F}=(\sqrt{\pi} / 2) \bar{\Lambda}=0.31 \pm 0.11 \mathrm{GeV}$. As a result, the range of Fermi motion parameter $p_{F}$ is thus 200 to $480 \mathrm{MeV}$.

- Fraction of $B \rightarrow K \ell^{+} \ell^{-}$and $B \rightarrow K^{*} \ell^{+} \ell^{-}$decays The signal model requires knowledge of the ratio between the branching fractions for exclusive $B \rightarrow K \ell^{+} \ell^{-}$and $B \rightarrow K^{*} \ell^{+} \ell^{-}$decays and inclusive $b \rightarrow s \ell^{+} \ell^{-}$decays. It relies on the calculations and uncertainties from Ref. [1], as shown in Table 2. We assume the branching fractions for $B \rightarrow K \ell^{+} \ell^{-}$and $B \rightarrow K^{*} \ell^{+} \ell^{-}$to be fully correlated (Gudrun Hiller indicates that these are more correlated than they are uncorrelated). We therefore vary both branching fractions together by $\pm 1 \sigma$.

- Transition point between $K^{*}$ to higher mass hadronic states

The signal model assumes that the hadronic system near the $K^{*}$ resonance is saturated by it below $M_{X_{s}}=1.1 \mathrm{GeV}$ (and above the kaon mass). The transition point is varied by $\pm 0.1 \mathrm{GeV}$.

\section{- Hadronization}

The inclusive Monte Carlo generator relies on Jetset to fragment and hadronize the system consisting of the final state $s$ quark and the spectator quark from the $B$ meson. Since the signal efficiencies depend strongly on the particle content of the final state, uncertainties in the fraction of charged and neutral kaon and pions translate into a significant uncertainty in the signal efficiency (for $m_{X_{s}}>1.1 \mathrm{GeV}$ ). We use measurements of various particle production observables (see below) performed in the semi-inclusive $B \rightarrow X_{s} \gamma$ BaBar 
analysis [31] to determine this uncertainty.

First, the ratio between modes containing a $K_{S}^{0}$ and those containing a charged $K$ is varied according to $K_{S}^{0} / K=0.50 \pm 0.05$, to account for possible isospin violation. The resulting relative uncertainty is $\pm 0.5 \%$ in both electron and muon channels. Second, the ratio between modes containing one $\pi^{0}$ and those containing none is varied according to $\pi^{0} / \pi=1.0 \pm 0.5$. Third, the ratio between two-body and three-body hadronic systems is varied according to $2-$ body $/ 3-$ body $=0.5 \pm 0.3$. The overall hadronization uncertainty in the electron and muon channels is $\pm 4.0 \%$ and $\pm 3.3 \%$, respectively.

The last two variations are motivated by the fact that the $b \rightarrow s \gamma$ data indicates the need for the fragmentation model to increase the ratio between modes including a $\pi^{0}$ and those without $\pi^{0}$ by a factor of 1.5 , and decrease the ratio between 2- and 3-body hadronic states by a factor of 0.4 .

\section{- Missing modes}

The 10 modes selected in this analysis only capture about $50 \%$ of the full set of final states. Approximately half of the missing modes is due to final states with a $K_{L}^{0}$ meson and can be determined from the $K_{S}^{0}$ modes. However, we need to account for the uncertainty in modes with too many pions or kaons (2 extra kaons may be produced via $s \bar{s}$ popping), as well as for modes with photons that do not originate from $\pi^{0}$ decays but rather from $\eta, \eta^{\prime}, \omega$, etc. The fraction of modes with $\geq 2 \pi^{0}$ is varied around the generator MC value of 0.20 (for $m_{X_{s}}>1.1 \mathrm{GeV} / c^{2}$ ) by $\pm 50 \%$.

The fraction of modes with either $0 \pi^{0}$ and $\geq 3$ charged pions or $1 \pi^{0}$ and $\geq 2$ charged pions is varied around the generator $\mathrm{MC}$ value of 0.26 (for $m_{X_{s}}>1.1$ $\left.\mathrm{GeV} / c^{2}\right)$ by $\pm 50 \%$. 
The fraction of modes with more than 1 kaon is varied around the generator $\mathrm{MC}$ value of 0.034 (for $m_{X_{s}}>1.1 \mathrm{GeV} / c^{2}$ ) by $\pm 50 \%$.

The fraction of modes with at least one photon that does not originate from the decay of a $\pi^{0}$ is varied around the generator MC value of 0.011 (for $\left.m_{X_{s}}>1.1 \mathrm{GeV} / c^{2}\right)$ by $\pm 50 \%$.

The overall systematic error due to missing mode uncertainties is $3.2 \%$ and $2.5 \%$ in the electron and muon modes, respectively.

- Monte Carlo statistics

The finite signal MC statistics translates into a relative uncertainty of $1.6 \%$, $1.8 \%$, and $1.3 \%$ in the electron, muon, and electron+muon channels.

- Number of $B \bar{B}$ events

This is taken to be $(88.9 \pm 1.0) \times 10^{6}$.

The signal model uncertainties amount to ${ }_{-16.3}^{+19.6} \%$ in the electron modes, ${ }_{-18.3}^{+20.8} \%$ in the muon modes, and ${ }_{-16.9}^{+19.9} \%$ in the combined $e+\mu$ modes. The other uncertainties in the efficiency amount to $\pm 9.9 \%, \pm 9.5 \%$, and $\pm 9.7 \%$, respectively. The total uncertainties in the signal efficiency is ${ }_{-19.1}^{+21.9} \%(e),{ }_{-20.7}^{+22.9} \%(\mu)$, and ${ }_{-19.5}^{+22.2 \%}(e+\mu)$. Relative uncertainties in the signal yields are $4.3 \%(e), 8.0 \%(\mu)$, and $4.4 \%(e+\mu)$. Tables 21, 22 and 10.1 summarize the results of the systematics studies. 
Table 21: List of systematic uncertainties (in \%) affecting the branching fraction calculation: uncertainties in the signal yield, the signal efficiency, and the number of $B$ mesons.

\begin{tabular}{lccc}
\hline \hline Source & $X_{s} e^{+} e^{-}$ & $X_{s} \mu^{+} \mu^{-}$ & $X_{s} \ell^{+} \ell^{-}$ \\
\hline \hline Signal shape & \pm 4.23 & \pm 2.77 & \pm 3.74 \\
Peaking bkg & \pm 0.78 & \pm 7.52 & \pm 2.25 \\
\hline \hline Signal yield total & \pm 4.31 & \pm 8.02 & \pm 4.36 \\
\hline \hline Tracking efficiency & \pm 4.10 & \pm 4.05 & \pm 4.08 \\
Lepton ID efficiency & \pm 1.70 & \pm 3.20 & \pm 2.17 \\
Kaon ID efficiency & \pm 0.76 & \pm 0.77 & \pm 0.76 \\
$\pi^{ \pm}$ID efficiency & \pm 0.94 & \pm 0.90 & \pm 0.93 \\
$K_{S}^{0}$ efficiency & \pm 0.85 & \pm 0.77 & \pm 0.82 \\
$\pi^{0}$ efficiency & \pm 0.67 & \pm 0.57 & \pm 0.64 \\
LR cut efficiency & \pm 3.86 & \pm 3.32 & \pm 3.69 \\
Cross-feed efficiency & \pm 5.53 & \pm 5.53 & \pm 5.53 \\
\hline Detector model subtotal & \pm 8.23 & \pm 8.40 & \pm 8.25 \\
\hline Fermi motion model & -1.04 & -1.09 & -1.06 \\
$\mathcal{B}\left(B \rightarrow K^{*} \ell^{+} \ell^{-}\right)$ & \pm 10.90 & \pm 9.92 & +16.59 \\
$K^{*}-X_{s}$ transition & \pm 4.68 & \pm 17.86 & \pm 16.30 \\
\hline Signal model subtotal & ${ }_{-16.30}^{19.58}$ & ${ }_{-18.35}^{+20.83}$ & \pm 4.49 \\
\hline Hadronization & \pm 4.05 & \pm 3.28 & \pm 3.95 \\
Missing modes & \pm 3.24 & \pm 2.52 & \pm 3.01 \\
MC statistics & \pm 1.63 & \pm 1.84 & \pm 1.26 \\
\hline \hline Efficiency total & -19.05 & -20.68 & -19.50 \\
\hline \hline$B \bar{B}$ counting & \pm 1.13 & \pm 1.13 & \pm 1.13 \\
\hline \hline
\end{tabular}


Table 22: List of systematic uncertainties (in \%) affecting the branching fraction calculation in the different hadronic mass ranges (see Table 19): uncertainties in the signal yield, the signal efficiency, and the number of $B$ mesons.

\begin{tabular}{lcccc}
\hline \hline Source & $m\left(X_{s}\right)$ bin 1 & $m\left(X_{s}\right)$ bin 2 & $m\left(X_{s}\right)$ bin 3 & $m\left(X_{s}\right)$ bin 4 \\
\hline \hline Signal shape & \pm 4.79 & \pm 0.72 & \pm 5.09 & \pm 3.31 \\
Peaking bkg & \pm 2.93 & \pm 1.77 & \pm 2.21 & \pm 1.98 \\
\hline \hline Signal yield total & \pm 5.61 & \pm 1.91 & \pm 5.55 & \pm 3.86 \\
\hline \hline Tracking efficiency & \pm 3.62 & \pm 4.42 & \pm 4.26 & \pm 4.55 \\
Lepton ID efficiency & \pm 2.22 & \pm 2.29 & \pm 2.15 & \pm 2.10 \\
Kaon ID efficiency & \pm 0.77 & \pm 0.83 & \pm 0.74 & \pm 0.76 \\
$\pi^{ \pm}$ID efficiency & \pm 0.88 & \pm 0.62 & \pm 1.00 & \pm 0.91 \\
$K_{S}^{0}$ efficiency & \pm 0.00 & \pm 1.24 & \pm 1.17 & \pm 1.61 \\
$\pi^{0}$ efficiency & \pm 0.00 & \pm 0.57 & \pm 0.87 & \pm 1.35 \\
LR cut efficiency & \pm 3.69 & \pm 3.69 & \pm 3.69 & \pm 3.69 \\
Cross-feed efficiency & \pm 1.93 & \pm 6.32 & \pm 8.54 & \pm 9.26 \\
\hline Detector model subtotal & \pm 6.06 & \pm 9.02 & \pm 10.63 & \pm 11.42 \\
\hline Fermi motion model & 0.00 & 0.00 & 0.00 & -0.44 \\
$\mathcal{B}\left(B \rightarrow K^{*} \ell^{+} \ell^{-}\right)$ & \pm .00 & \pm 0.00 & \pm 0.00 & \pm 2.34 \\
$K^{*}-X_{s}$ transition & \pm 0.00 & \pm 0.00 & \pm 0.01 & \pm 10.61 \\
\hline Signal model subtotal & ${ }_{-0.00}^{+0.00}$ & ${ }_{-0.00}^{+0.00}$ & ${ }_{-0.01}^{+0.01}$ & -10.87 \\
\hline Hadronization & \pm 0.00 & \pm 0.00 & \pm 0.00 & \pm 17.08 \\
Missing modes & \pm 0.00 & \pm 0.00 & \pm 0.00 & \pm 12.62 \\
MC statistics & \pm 0.47 & \pm 8.44 & \pm 1.35 & \pm 5.37 \\
\hline \hline Efficiency total & ${ }_{-6.08}^{+6.08}$ & ${ }_{-12.35}$ & ${ }_{-10.71}$ & -26.99 \\
\hline \hline$B \bar{B}$ counting & \pm 1.12 & \pm 1.12 & \pm 1.12 & \pm 1.12 \\
\hline \hline
\end{tabular}




\subsection{CP asymmetry systematics}

All systematic errors cancel by definition in the measurement of the $C P$ asymmetry, except for systematic effects which are different for $b$ and $\bar{b}$ final states. The effect of these differences is to fake a $C P$ asymmetry. There are many known effects which can give rise to the fake $C P$ asymmetry. We determine the systematic uncertainty using the charmonium veto sample. As a cross check, we also study the impact of individual detector modeling uncertainties.

In the following section, we will first describe the charmonium veto sample method and then the itemized method.

\section{Charmonium veto sample method}

We use the charmonium veto sample to estimate the $C P$ asymmetry systematic uncertainty. We consider the difference in the peaking component of $B$ and $\bar{B}$ charmonium veto sample as the uncertainty, assuming the $C P$ asymmetry in $B$ decays to charmonium to be zero. The advantage of this method is that since the uncertainty is directly estimated from data, all effects that may fake a $C P$ asymmetry are included, except mis-ID biases (however this effect is very small as

described later). This is of course only strictly valid in the dilepton mass regions of the charmonium veto cuts but we assume this restricted range to be representative of the full range included in the analysis. The result of separate fits to the $B$ and $\bar{B}$ data is shown in Fig. 75. We find the bias to be $A_{C P}(J / \psi \psi(2 S))=-0.005 \pm 0.016$, where the error is statistical only. 

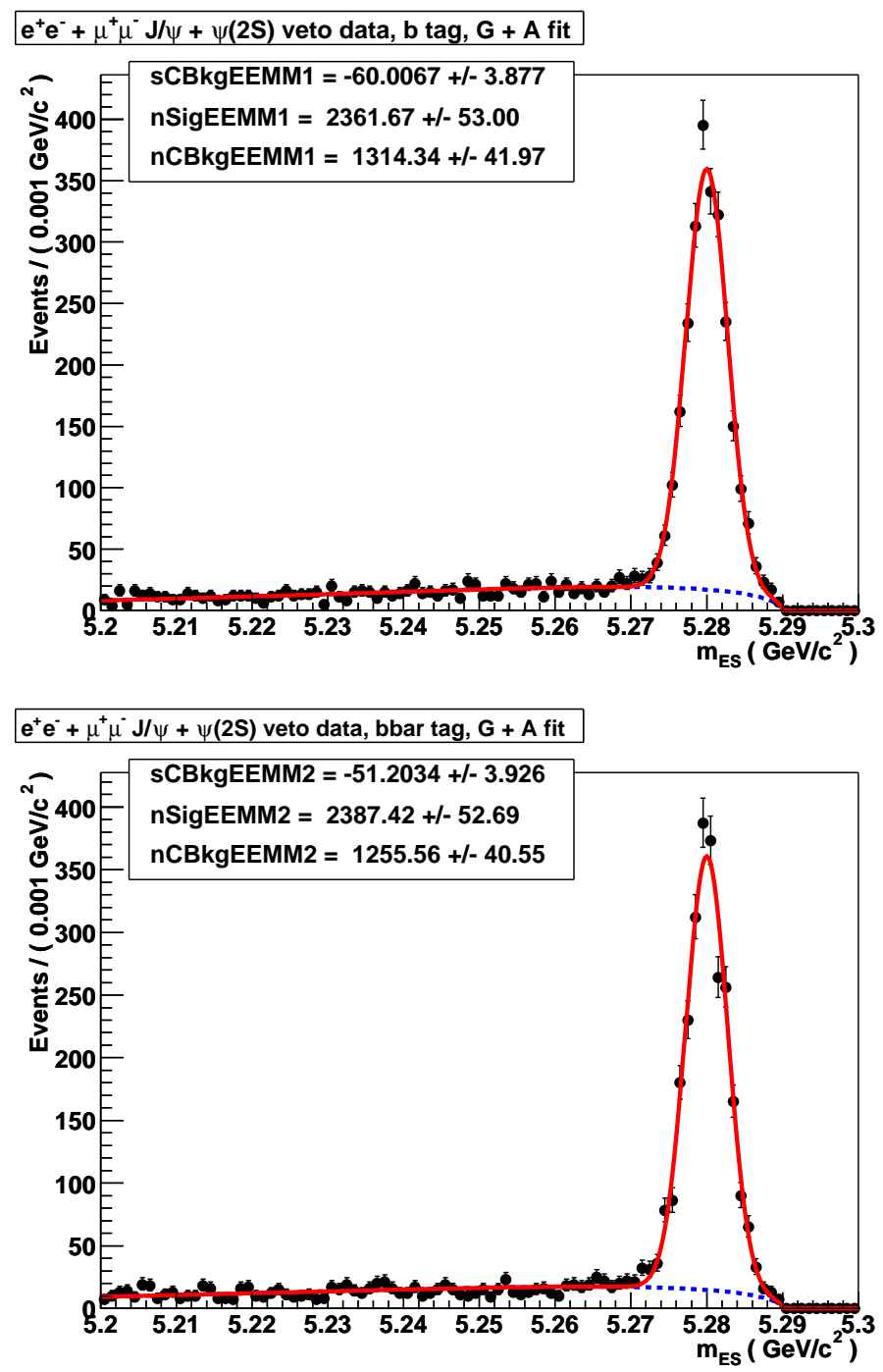

Figure 75: Distributions of $m_{E S}$ for combined electron and muon data in selftagged $\bar{B}$ (top) and $B$ (bottom) decays from the charmonium veto sample. The red line is the result of the fit, with the total background component shown in the dashed blue line. 
Table 23: Apparent $C P$ asymmetry in reconstructed signal events due to tracking, kaon ID and pion ID efficiencies biases. The corresponding statistical error and the error due to tracking, kaon ID and pion ID efficiencies errors are shown for the computed $C P$ asymmetry.

\begin{tabular}{cr} 
& $A_{C P}$ \\
\hline Trk eff. & $0.0094 \pm 0.0076 \pm 0.0017$ \\
Kaon ID & $0.0027 \pm 0.0076 \pm 0.0002$ \\
Pion ID & $0.0002 \pm 0.0076 \pm 0.0016$
\end{tabular}

\section{Itemized method}

We will first describe the fake $C P$ asymmetry due to detector biases which affects the signal events, then we will describe the mis-tagged events and the corresponding asymmetry and finally we will investigate the $C P$ asymmetry in cross-feed events.

- $C P$ asymmetry in signal events due to efficiency biases The detector efficiency is not perfectly symmetric for positive and negative charged tracks. Tracking efficiency differences can arise from the opposite curvature of the tracks in the solenoidal field. In the Drift Chamber this couples to the drift electron direction, which is distorted by the Lorentz angle effect. This can affect both the track reconstruction and the $\mathrm{dE} / \mathrm{dx}$ particle identification information. The other major source of asymmetry is the different strong interaction rates of positive and negative particles. This leads to tracking efficiency asymmetries and particle identification asymmetries.

We use the efficiency tables for positive and negative charged tracks, kaons and electrons, provided by the Tracking Efficiency Task Force, to analyze the apparent $C P$ asymmetry due to the particle reconstruction bias. The results are shown in Table 23. There is no evidence of bias within the errors.

- $C P$ asymmetry in mis-tagged signal MC events due to mis-ID biases 
Some signal events are not properly tagged in the correct flavor due to detector effects. This can generate a fake $C P$ asymmetry. We study only those events that are still classified as "true" signal events, i.e. reconstructed in the same or opposite flavor. Events reconstructed with opposite flavor are what we call "mistagged" events.

In the following, first we study the mis-tag rate in our events, then we investigate the corresponding flavor asymmetry.

We estimate the mis-tag rate using reconstructed signal $\mathrm{MC}$ event. The mis-tag rate is defined as the percentage of times the wrong $b$-flavor is reconstructed with respect to all the events which pass the selection. We find the mis-tagged rate $\alpha_{\text {tag }}=0.0002 \pm 0.0076$, where the error is statistical only. The mis-tagged rate is very small.

In order to estimate the flavor asymmetry in the misidentified events, we compute the asymmetry of the wrongly reconstructed $b$ flavor events versus the wrongly reconstructed $\bar{b}$ events, normalized by the total events which pass the selection. The result is $A_{C P}$ (Mis-tagged rate) $=-0.0002 \pm 0.0076$, where the error is statistical only. The asymmetry is consistent with zero. We also check the fake $C P$ asymmetry in mis-tagged events due to $K^{ \pm} \leftrightarrow \pi^{ \pm}$and $K^{ \pm} \leftrightarrow \mu^{ \pm}$mis-IDs by using PID look up tables for positive and negative charged particles. The results are summarized in Table 24. The asymmetries are zero.

- $C P$ asymmetry in cross-feed MC events due to detector effects There can also be a $C P$ asymmetry in the cross-feed events due to detector biases. In this section we consider only the "peaking" component of the cross-feed. There are no recipes available for estimating the rates for replacing or adding 
Table 24: Fake $A_{C P}$ in mis-tagged events due to $K^{ \pm} \leftrightarrow \pi^{ \pm}$and $K^{ \pm} \leftrightarrow \mu^{ \pm}$ mis-IDs. The corresponding statistical error and the error due to $K^{ \pm} \leftrightarrow \pi^{ \pm}$and $K^{ \pm} \leftrightarrow \mu^{ \pm}$mis-ID errors are shown for the computed $C P$ asymmetry.

$$
\begin{array}{lr} 
& A_{C P} \\
\hline K^{ \pm} \leftrightarrow \pi^{ \pm} \text {mis-id } & -0.0000 \pm 0.0076 \pm 0.0000 \\
K^{ \pm} \leftrightarrow \mu^{ \pm} \text {mis-id } & -0.0000 \pm 0.0076 \pm 0.0000
\end{array}
$$

\begin{tabular}{cc} 
Systematic & \\
\hline Trk eff. & 1.12 \\
Kaon ID & 0.28 \\
Pion ID & 0.18 \\
Mis-tag rate & 0.02 \\
$K^{ \pm} \leftrightarrow \pi^{ \pm}$mis-id & 0.00 \\
$K^{ \pm} \leftrightarrow \mu^{ \pm}$mis-id & 0.00 \\
MC stat & 0.76 \\
\hline Total & 1.49
\end{tabular}

Table 25: List of fractional systematic uncertainties (in \%) affecting the $A_{C P}$ calculation.

particles, so we use the peaking component of the cross-feed events from the reconstructed signal MC samples. Result of the difference in the peaking component of $b$ and $\bar{b}$ cross-feed events is considered as a bias of the asymmetry. We obtained the bias from the cross-feed events to be $A_{C P}($ cross-feed $)=$ $0.14 \pm 0.18$., where the error is statistical only. As the statistical error is very large (i.e. we do not expect many events from the peaking background components) we do not observe any asymmetry.

We summarize the list of systematic uncertainties for $C P$ asymmetry with the itemized method in Table 25. 


\section{H A P T E R 11}

\section{Summary}

We have observed the inclusive $b \rightarrow s \ell^{+} \ell^{-}$process in the $B \rightarrow X_{s} e^{+} e^{-}$and $B \rightarrow X_{s} \mu^{+} \mu^{-}$channels with a sum of exclusive modes technique. The inclusive branching fraction is determined to be

$$
\mathcal{B}\left(B \rightarrow X_{s} \ell^{+} \ell^{-}\right)=(5.6 \pm 1.5(\text { stat }) \pm 0.6(\text { exp syst }) \pm 1.1(\text { model syst })) \times 10^{-6}
$$

for $m_{l l}>0.2 \mathrm{GeV}$. Tables 26 and 27 summarize the other results of the analysis. These agree with the theoretical prediction of $(4.2 \pm 0.7) \times 10^{-6}[32]$ for $m_{\ell \ell}>0.2$ $\mathrm{GeV},(1.63 \pm 0.20) \times 10^{-6}[33]$ for $1<m_{\ell \ell}<2.45 \mathrm{GeV}$, and $(0.40 \pm 0.08) \times$ $10^{-6}$ [33] for $m_{\ell \ell}>3.795 \mathrm{GeV}$. We determine the direct $\mathrm{CP}$ asymmetry to be $A_{C P}=-0.22 \pm 0.26($ stat $) \pm 0.02($ syst $)$, in agreement with the vanishingly small asymmetry expected in the SM [25].

Figure 76 shows the results in terms of number of signal events (errors are statistical only) as a function of hadronic mass and dilepton mass. The figure shows that higher mass hadronic states contribute a significant portion of the total signal. Similarly, Fig. 77 shows the differential branching fraction as a function of hadronic and dilepton mass. In this case, systematic uncertainties in the branching fractions are included in quadrature in the outermost vertical error bars. 
Table 26: Summary of results: signal yield, signal significance, signal efficiency and branching fraction. In the case of the signal yield, the first error is statistical and the second error is systematic. In the case of the signal efficiency, the first error corresponds to the experimental systematic uncertainty arising from detector modeling, hadronization, $B \bar{B}$ counting, and Monte Carlo statistics, whereas the second error corresponds to the uncertainties in the signal model. In the case of the branching fraction, the first, second, and third errors correspond to statistical, experimental systematic, and signal model systematic uncertainties, respectively.

\begin{tabular}{lcccc}
\hline Sample & $N_{\text {sig }}$ & Signif. & $\epsilon(\%)$ & $\mathcal{B}\left(\times 10^{-6}\right)$ \\
\hline \hline$X_{s} e^{+} e^{-}$ & $29.22 \pm 8.28 \pm 1.26$ & 4.0 & $2.74 \pm 0.27 \pm 0.49$ & $6.00 \pm 1.70 \pm 0.65 \pm 1.09$ \\
$X_{s} \mu^{+} \mu^{-}$ & $11.25 \pm 6.19 \pm 0.90$ & 2.0 & $1.26 \pm 0.12 \pm 0.25$ & $5.04 \pm 2.77 \pm 0.63 \pm 1.01$ \\
$X_{s} \ell^{+} \ell^{-}$ & $40.06 \pm 10.35 \pm 1.75$ & 4.3 & $2.00 \pm 0.19 \pm 0.37$ & $5.64 \pm 1.46 \pm 0.60 \pm 1.06$ \\
\hline
\end{tabular}

Table 27: Summary of branching fractions, where the first error is statistical, the second is systematic excluding signal model uncertainties, and the third is from signal model systematics.

\begin{tabular}{|c|c|c|}
\hline Sample & $\overline{\mathcal{B}\left(\times 10^{-6}\right)}$ & Total $\sigma_{\text {syst }}$ \\
\hline 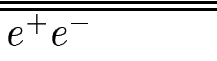 & 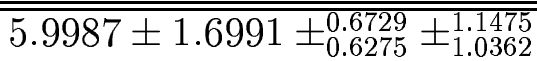 & \\
\hline$\mu^{+} \mu^{-}$ & $5.0373 \pm 2.7742 \pm_{0.6164}^{0.6459} \pm_{0.9099}^{1.1172}$ & $\pm_{1.0990}^{1.2905}$ \\
\hline$\ell^{+} \ell^{-}$ & $5.6396 \pm 1.4571 \pm_{0.5813}^{0.6222} \pm_{0.9872}^{1.1312}$ & $\pm_{1.1458}^{1.2910}$ \\
\hline \multicolumn{3}{|c|}{$m\left(X_{s}\right)\left(\mathrm{GeV} / c^{2}\right)$} \\
\hline $0.4-0.6$ & $0.5279 \pm 0.1719 \pm_{0.0434}^{0.0449} \pm_{0.0000}^{0.0000}$ & $\pm_{0.0434}^{0.0449}$ \\
\hline $0.6-0.8$ & $0.3223 \pm 0.2407 \pm_{0.00380}^{0.0432} \pm_{0.0000}^{0.0000}$ & $\pm_{0.0380}^{0.0432}$ \\
\hline $0.8-1.0$ & $0.6442 \pm 0.4018 \pm_{0.0743}^{0.0826} \pm_{0.0001}^{0.0001}$ & $\pm_{0.0743}^{0.0826}$ \\
\hline $1.0-1.8$ & $3.4960 \pm 2.0605 \pm_{0.7769}^{1.0092} \pm_{0.4240}^{0.4234}$ & $\pm_{0.8851}^{1.0944}$ \\
\hline \multicolumn{3}{|c|}{$m\left(\ell^{+} \ell^{-}\right)\left(\mathrm{GeV} / c^{2}\right)$} \\
\hline $0.2-1.0$ & $0.0776 \pm 0.3650 \pm_{0.00267}^{0.0644} \pm_{0.0271}^{0.0209}$ & $\pm_{0.0381}^{0.0677}$ \\
\hline $1.0-2.0$ & $1.6343 \pm 0.6354 \pm_{0.1849}^{0.1988} \pm_{0.4704}^{0.4097}$ & $\pm_{0.5054}^{0.4554}$ \\
\hline $2.0-m_{J / \psi}$ & $1.7508 \pm 0.8394 \pm_{0.1921}^{0.2036} \pm_{0.2675}^{0.3396}$ & $\pm_{0.3293}^{0.3960}$ \\
\hline$m_{J / \psi}-m_{\psi^{\prime}}$ & $0.9786 \pm 0.8450 \pm_{0.1076}^{0.162} \pm_{0.1161}^{0.1895}$ & $\pm_{0.1582}^{0.2223}$ \\
\hline$m_{\psi^{\prime}}-5.0$ & $0.6356 \pm 0.3175 \pm_{0.0793}^{0.0894} \pm_{0.0416}^{0.0809}$ & $\pm_{0.0895}^{0.1206}$ \\
\hline $1.0-2.45$ & $1.8466 \pm 0.7163 \pm_{0.2127}^{0.2268} \pm_{0.4666}^{0.4452}$ & $\pm_{0.5128}^{0.4996}$ \\
\hline $3.795-5.0$ & $0.4976 \pm 0.2486 \pm_{0.0016}^{0.0695} \pm_{0.0239}^{0.0462}$ & $\pm_{0.0661}^{0.0835}$ \\
\hline
\end{tabular}



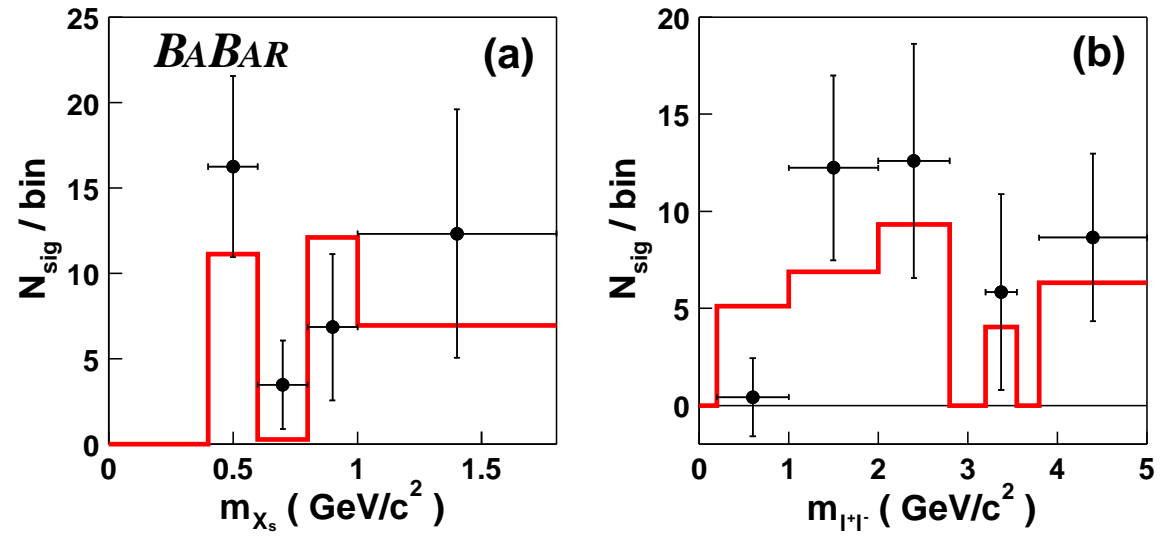

Figure 76: Number of fitted signal events in bins of $m_{X s}$ (left) and $m_{l l}$ for electron and muon channels combined for data (points) and MC signal (histogram). The error bars represent the statistical uncertainty only.
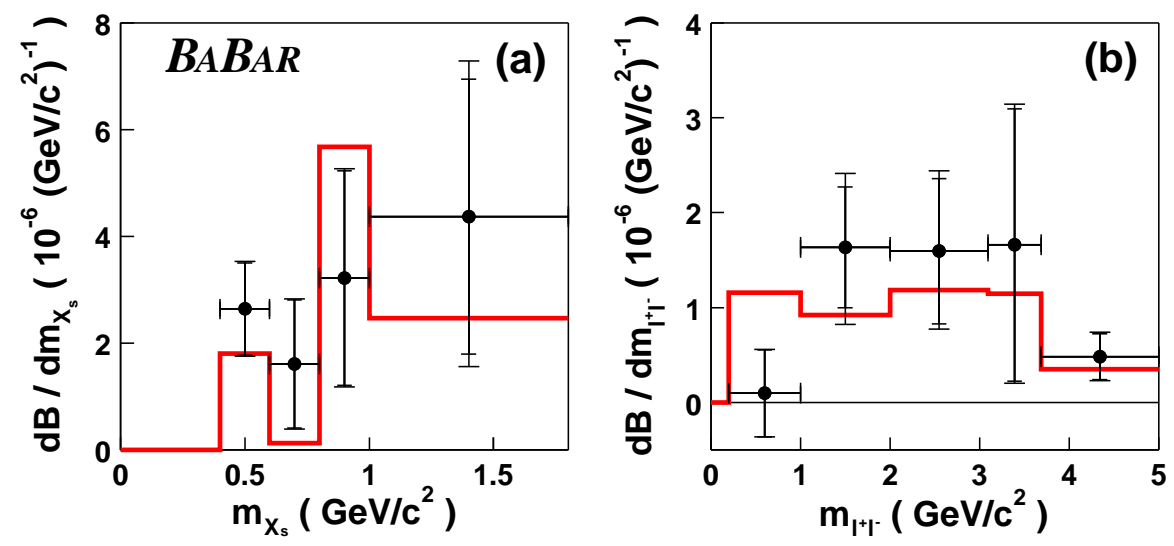

Figure 77: Differential branching fraction as a function of hadronic mass (left) and dilepton mass (right), averaged over electron and muon channels for data (points) and signal Monte Carlo (histogram). 
032796

\section{A P P E N D I X A}

\section{Signal efficiency tables}

The tables in this section show the MC signal efficiency for different stages of the analysis. The numbers correspond to number of events in $81.9 \mathrm{fb}^{-1}$. The entries in [\%] show the efficiency of the corresponding step. The last column lists the overall efficiencies. 


\begin{tabular}{|c|c|c|c|c|c|c|c|c|c|c|}
\hline Topol & gen & skim + prVts & in lists & preSel & lept Sel & had Sel & postSel & jpsi veto & LR cut & tot Eff \\
\hline other & 298.102 & $\begin{array}{c}279.981 \\
93.921 \%\end{array}$ & $\begin{array}{c}56.539 \\
20.194 \%\end{array}$ & $\begin{array}{c}1.101 \\
1.947 \%\end{array}$ & $\begin{array}{c}0.900 \\
81.793 \%\end{array}$ & $\begin{array}{c}0.000 \\
0.000 \%\end{array}$ & $\begin{array}{c}0.000 \\
0.000 \%\end{array}$ & $\begin{array}{c}0.000 \\
0.000 \%\end{array}$ & $\begin{array}{c}0.000 \\
0.000 \%\end{array}$ & $0.000 \%$ \\
\hline K & 3.405 & $\begin{array}{c}32.104 \\
96.107 \%\end{array}$ & $\begin{array}{c}17.795 \\
55.430 \%\end{array}$ & $\begin{array}{c}14.977 \\
84.164 \%\end{array}$ & $\begin{array}{c}14.590 \\
97.415 \%\end{array}$ & $\begin{array}{c}13.661 \\
93.633 \%\end{array}$ & $\begin{array}{c}11.250 \\
82.348 \%\end{array}$ & $\begin{array}{c}7.256 \\
64.502 \%\end{array}$ & $\begin{array}{c}5.588 \\
77.009 \%\end{array}$ & $16.728 \%$ \\
\hline $\mathrm{K} \mathrm{pi0}$ & 53.557 & $\begin{array}{c}51.498 \\
96.155 \%\end{array}$ & $\begin{array}{c}8.238 \\
15.997 \%\end{array}$ & $\begin{array}{r}6.866 \\
8.344 \%\end{array}$ & $\begin{array}{c}6.315 \\
91.972 \%\end{array}$ & $\begin{array}{c}4.637 \\
73.431 \%\end{array}$ & $\begin{array}{c}3.778 \\
81.476 \%\end{array}$ & $\begin{array}{c}2.482 \\
65.687 \%\end{array}$ & $\begin{array}{c}1.361 \\
54.825 \%\end{array}$ & $2.540 \%$ \\
\hline K pi & 95.148 & $\begin{array}{c}90.858 \\
95.491 \%\end{array}$ & $\begin{array}{c}35.978 \\
39.598 \%\end{array}$ & $\begin{array}{c}29.011 \\
80.635 \%\end{array}$ & $\begin{array}{c}27.333 \\
94.215 \%\end{array}$ & $\begin{array}{c}20.876 \\
76.377 \%\end{array}$ & $\begin{array}{c}16.619 \\
79.609 \%\end{array}$ & $\begin{array}{c}10.781 \\
64.873 \%\end{array}$ & $\begin{array}{c}6.410 \\
59.458 \%\end{array}$ & $6.737 \%$ \\
\hline K pi pi0 & 42.139 & $\begin{array}{c}40.451 \\
95.994 \%\end{array}$ & $\begin{array}{c}4.639 \\
11.468 \%\end{array}$ & $\begin{array}{c}3.707 \\
79.913 \%\end{array}$ & $\begin{array}{r}3.270 \\
8.203 \%\end{array}$ & $\begin{array}{c}1.997 \\
61.063 \%\end{array}$ & $\begin{array}{c}1.379 \\
69.073 \%\end{array}$ & $\begin{array}{c}1.069 \\
77.521 \%\end{array}$ & $\begin{array}{c}0.419 \\
39.189 \%\end{array}$ & $0.994 \%$ \\
\hline K pi pi & 37.254 & $\begin{array}{c}35.066 \\
94.127 \%\end{array}$ & $\begin{array}{c}10.132 \\
28.895 \%\end{array}$ & $\begin{array}{c}7.717 \\
76.157 \%\end{array}$ & $\begin{array}{c}6.979 \\
90.438 \%\end{array}$ & $\begin{array}{c}4.592 \\
65.804 \%\end{array}$ & $\begin{array}{c}3.282 \\
71.460 \%\end{array}$ & $\begin{array}{c}2.509 \\
76.454 \%\end{array}$ & $\begin{array}{c}1.109 \\
44.195 \%\end{array}$ & $2.976 \%$ \\
\hline K pi pi pi0 & 27.568 & $\begin{array}{c}25.785 \\
93.532 \%\end{array}$ & $\begin{array}{c}1.391 \\
5.393 \%\end{array}$ & $\begin{array}{c}1.020 \\
73.331 \%\end{array}$ & $\begin{array}{c}0.866 \\
84.882 \%\end{array}$ & $\begin{array}{c}0.421 \\
48.614 \%\end{array}$ & $\begin{array}{c}0.209 \\
49.653 \%\end{array}$ & $\begin{array}{c}0.177 \\
84.770 \%\end{array}$ & $\begin{array}{c}0.000 \\
0.000 \%\end{array}$ & $0.000 \%$ \\
\hline K pi pi pi & 7.853 & $\begin{array}{c}7.198 \\
91.653 \%\end{array}$ & $\begin{array}{c}1.294 \\
17.983 \%\end{array}$ & $\begin{array}{c}0.854 \\
65.996 \%\end{array}$ & $\begin{array}{c}0.699 \\
81.841 \%\end{array}$ & $\begin{array}{c}0.414 \\
59.260 \%\end{array}$ & $\begin{array}{c}0.187 \\
45.098 \%\end{array}$ & $\begin{array}{c}0.144 \\
77.009 \%\end{array}$ & $\begin{array}{c}0.000 \\
0.000 \%\end{array}$ & $0.000 \%$ \\
\hline $\mathrm{Ks}+\mathrm{K} 1$ & 30.880 & $\begin{array}{c}29.873 \\
96.739 \%\end{array}$ & $\begin{array}{c}4.822 \\
16.143 \%\end{array}$ & $\begin{array}{c}4.206 \\
87.209 \%\end{array}$ & $\begin{array}{c}4.087 \\
97.170 \%\end{array}$ & $\begin{array}{c}3.816 \\
93.389 \%\end{array}$ & $\begin{array}{c}3.157 \\
82.728 \%\end{array}$ & $\begin{array}{c}2.017 \\
63.875 \%\end{array}$ & $\begin{array}{c}1.519 \\
75.304 \%\end{array}$ & $4.918 \%$ \\
\hline Ks pi0 & 24.119 & $\begin{array}{c}23.172 \\
96.075 \%\end{array}$ & $\begin{array}{c}2.110 \\
9.105 \%\end{array}$ & $\begin{array}{r}1.873 \\
8.763 \%\end{array}$ & $\begin{array}{c}1.794 \\
95.798 \%\end{array}$ & $\begin{array}{c}1.207 \\
67.294 \%\end{array}$ & $\begin{array}{c}0.959 \\
79.427 \%\end{array}$ & $\begin{array}{c}0.625 \\
65.163 \%\end{array}$ & $\begin{array}{c}0.353 \\
56.481 \%\end{array}$ & $1.463 \%$ \\
\hline Ks pi & 52.629 & $\begin{array}{c}50.269 \\
95.516 \%\end{array}$ & $\begin{array}{c}10.699 \\
21.283 \%\end{array}$ & $\begin{array}{c}8.867 \\
82.883 \%\end{array}$ & $\begin{array}{c}8.288 \\
93.471 \%\end{array}$ & $\begin{array}{c}6.297 \\
75.972 \%\end{array}$ & $\begin{array}{c}5.127 \\
81.426 \%\end{array}$ & $\begin{array}{c}3.353 \\
65.386 \%\end{array}$ & $\begin{array}{c}2.074 \\
61.861 \%\end{array}$ & $3.941 \%$ \\
\hline Ks pi pi0 & 23.240 & $\begin{array}{c}22.187 \\
95.469 \%\end{array}$ & $\begin{array}{c}1.425 \\
6.422 \%\end{array}$ & $\begin{array}{c}1.168 \\
81.976 \%\end{array}$ & $\begin{array}{c}1.027 \\
87.943 \%\end{array}$ & $\begin{array}{c}0.580 \\
56.428 \%\end{array}$ & $\begin{array}{c}0.419 \\
72.210 \%\end{array}$ & $\begin{array}{c}0.316 \\
75.423 \%\end{array}$ & $\begin{array}{c}0.111 \\
35.040 \%\end{array}$ & $0.476 \%$ \\
\hline Ks pi pi & 17.396 & $\begin{array}{c}16.310 \\
93.758 \%\end{array}$ & $\begin{array}{c}2.709 \\
16.611 \%\end{array}$ & $\begin{array}{c}2.212 \\
81.633 \%\end{array}$ & $\begin{array}{c}2.023 \\
91.483 \%\end{array}$ & $\begin{array}{c}1.357 \\
67.064 \%\end{array}$ & $\begin{array}{c}0.955 \\
70.390 \%\end{array}$ & $\begin{array}{c}0.770 \\
80.617 \%\end{array}$ & $\begin{array}{c}0.303 \\
39.334 \%\end{array}$ & $1.741 \%$ \\
\hline Ks pi pi pi0 & 11.655 & $\begin{array}{c}10.934 \\
93.817 \%\end{array}$ & $\begin{array}{c}0.255 \\
2.337 \%\end{array}$ & $\begin{array}{c}0.220 \\
86.259 \%\end{array}$ & $\begin{array}{c}0.198 \\
90.043 \%\end{array}$ & $\begin{array}{c}0.100 \\
50.572 \%\end{array}$ & $\begin{array}{c}0.044 \\
43.739 \%\end{array}$ & $\begin{array}{c}0.044 \\
100.000 \%\end{array}$ & $\begin{array}{c}0.000 \\
0.000 \%\end{array}$ & $0.000 \%$ \\
\hline Ks pi pi pi & 4.452 & $\begin{array}{c}4.114 \\
92.412 \%\end{array}$ & $\begin{array}{c}0.462 \\
11.242 \%\end{array}$ & $\begin{array}{c}0.309 \\
66.775 \%\end{array}$ & $\begin{array}{c}0.264 \\
85.329 \%\end{array}$ & $\begin{array}{c}0.170 \\
64.335 \%\end{array}$ & $\begin{array}{c}0.078 \\
46.128 \%\end{array}$ & $\begin{array}{c}0.060 \\
77.208 \%\end{array}$ & $\begin{array}{c}0.000 \\
0.000 \%\end{array}$ & $0.000 \%$ \\
\hline Total & 759.397 & $\begin{array}{c}719.800 \\
94.786 \%\end{array}$ & $\begin{array}{r}158.490 \\
22.019 \%\end{array}$ & $\begin{array}{r}84.107 \\
53.068 \%\end{array}$ & $\begin{array}{r}78.633 \\
93.491 \%\end{array}$ & $\begin{array}{r}60.125 \\
76.463 \%\end{array}$ & $\begin{array}{r}47.443 \\
78.906 \%\end{array}$ & $\begin{array}{c}31.602 \\
66.612 \%\end{array}$ & $\begin{array}{r}19.246 \\
60.899 \%\end{array}$ & .534 \\
\hline
\end{tabular}




\begin{tabular}{|c|c|c|c|c|c|c|c|c|c|c|c|}
\hline & Topol & gen & skim + prVtx & in lists & preSel & lept Sel & had Sel & postSel & jpsi veto & LR cut & tot Eff \\
\hline & other & 295.036 & $\begin{array}{l}278.068 \\
94.249 \%\end{array}$ & $\begin{array}{l}18.516 \\
6.659 \%\end{array}$ & $\begin{array}{c}0.385 \\
2.079 \%\end{array}$ & $\begin{array}{c}0.282 \\
73.360 \%\end{array}$ & $\begin{array}{c}0.000 \\
0.000 \%\end{array}$ & $\begin{array}{c}0.000 \\
0.000 \%\end{array}$ & $\begin{array}{c}0.000 \\
0.000 \%\end{array}$ & $\begin{array}{c}0.000 \\
0.000 \%\end{array}$ & $0.000 \%$ \\
\hline & K & 33.422 & $\begin{array}{c}32.125 \\
96.119 \%\end{array}$ & $\begin{array}{c}6.884 \\
21.430 \%\end{array}$ & $\begin{array}{c}6.446 \\
93.640 \%\end{array}$ & $\begin{array}{c}5.886 \\
91.298 \%\end{array}$ & $\begin{array}{c}5.673 \\
96.386 \%\end{array}$ & $\begin{array}{c}4.625 \\
81.535 \%\end{array}$ & $\begin{array}{c}3.441 \\
74.393 \%\end{array}$ & $\begin{array}{c}2.968 \\
86.268 \%\end{array}$ & $8.881 \%$ \\
\hline & $\mathrm{K}$ pi0 & 54.157 & $\begin{array}{c}52.071 \\
96.148 \%\end{array}$ & $\begin{array}{c}2.946 \\
5.658 \%\end{array}$ & $\begin{array}{c}2.746 \\
93.211 \%\end{array}$ & $\begin{array}{c}2.286 \\
83.245 \%\end{array}$ & $\begin{array}{c}1.854 \\
81.110 \%\end{array}$ & $\begin{array}{c}1.584 \\
85.428 \%\end{array}$ & $\begin{array}{c}1.125 \\
71.001 \%\end{array}$ & $\begin{array}{c}0.592 \\
52.625 \%\end{array}$ & $1.093 \%$ \\
\hline & K pi & 95.483 & $\begin{array}{c}91.194 \\
95.508 \%\end{array}$ & $\begin{array}{c}12.732 \\
13.961 \%\end{array}$ & $\begin{array}{c}11.340 \\
89.067 \%\end{array}$ & $\begin{array}{c}9.780 \\
86.241 \%\end{array}$ & $\begin{array}{c}8.391 \\
85.801 \%\end{array}$ & $\begin{array}{c}6.765 \\
80.627 \%\end{array}$ & $\begin{array}{c}4.857 \\
71.788 \%\end{array}$ & $\begin{array}{c}2.825 \\
58.164 \%\end{array}$ & $2.959 \%$ \\
\hline & K pi pi0 & 40.036 & $\begin{array}{c}38.495 \\
96.152 \%\end{array}$ & $\begin{array}{c}1.385 \\
3.599 \%\end{array}$ & $\begin{array}{c}1.224 \\
88.365 \%\end{array}$ & $\begin{array}{c}0.883 \\
72.104 \%\end{array}$ & $\begin{array}{c}0.666 \\
75.443 \%\end{array}$ & $\begin{array}{c}0.488 \\
73.202 \%\end{array}$ & $\begin{array}{c}0.367 \\
75.380 \%\end{array}$ & $\begin{array}{c}0.146 \\
39.851 \%\end{array}$ & $0.366 \%$ \\
\hline & K pi pi & 38.799 & $\begin{array}{c}36.632 \\
94.415 \%\end{array}$ & $\begin{array}{c}2.967 \\
8.100 \%\end{array}$ & $\begin{array}{c}2.462 \\
82.964 \%\end{array}$ & $\begin{array}{c}1.867 \\
75.847 \%\end{array}$ & $\begin{array}{c}1.434 \\
76.796 \%\end{array}$ & $\begin{array}{c}1.118 \\
77.957 \%\end{array}$ & $\begin{array}{c}0.869 \\
77.716 \%\end{array}$ & $\begin{array}{c}0.388 \\
44.627 \%\end{array}$ & $0.999 \%$ \\
\hline & K pi pi pi0 & 27.086 & $\begin{array}{c}25.371 \\
93.666 \%\end{array}$ & $\begin{array}{c}0.470 \\
1.853 \%\end{array}$ & $\begin{array}{c}0.366 \\
77.809 \%\end{array}$ & $\begin{array}{c}0.253 \\
69.080 \%\end{array}$ & $\begin{array}{c}0.168 \\
66.630 \%\end{array}$ & $\begin{array}{c}0.106 \\
63.003 \%\end{array}$ & $\begin{array}{c}0.091 \\
85.364 \%\end{array}$ & $\begin{array}{c}0.000 \\
0.000 \%\end{array}$ & $0.000 \%$ \\
\hline & K pi pi pi & 7.690 & $\begin{array}{c}7.049 \\
91.664 \%\end{array}$ & $\begin{array}{c}0.423 \\
6.000 \%\end{array}$ & $\begin{array}{c}0.367 \\
86.667 \%\end{array}$ & $\begin{array}{c}0.298 \\
81.373 \%\end{array}$ & $\begin{array}{c}0.189 \\
63.237 \%\end{array}$ & $\begin{array}{r}0.104 \\
5.172 \%\end{array}$ & $\begin{array}{c}0.084 \\
80.248 \%\end{array}$ & $\begin{array}{c}0.000 \\
0.000 \%\end{array}$ & $0.000 \%$ \\
\hline & $\mathrm{Ks}+\mathrm{K} 1$ & 30.866 & $\begin{array}{c}29.891 \\
96.842 \%\end{array}$ & $\begin{array}{c}1.831 \\
6.126 \%\end{array}$ & $\begin{array}{c}1.754 \\
95.789 \%\end{array}$ & $\begin{array}{c}1.576 \\
89.840 \%\end{array}$ & $\begin{array}{c}1.525 \\
96.774 \%\end{array}$ & $\begin{array}{c}1.261 \\
82.676 \%\end{array}$ & $\begin{array}{c}0.936 \\
74.255 \%\end{array}$ & $\begin{array}{c}0.796 \\
85.021 \%\end{array}$ & $2.579 \%$ \\
\hline & Ks pi0 & 24.368 & $\begin{array}{c}23.462 \\
96.283 \%\end{array}$ & $\begin{array}{c}0.664 \\
2.830 \%\end{array}$ & $\begin{array}{c}0.639 \\
96.201 \%\end{array}$ & $\begin{array}{c}0.532 \\
83.268 \%\end{array}$ & $\begin{array}{c}0.418 \\
78.494 \%\end{array}$ & $\begin{array}{c}0.325 \\
77.936 \%\end{array}$ & $\begin{array}{c}0.222 \\
68.120 \%\end{array}$ & $\begin{array}{c}0.120 \\
54.114 \%\end{array}$ & $0.492 \%$ \\
\hline & Ks pi & 52.470 & $\begin{array}{c}50.230 \\
95.731 \%\end{array}$ & $\begin{array}{c}3.813 \\
7.591 \%\end{array}$ & $\begin{array}{c}3.511 \\
92.066 \%\end{array}$ & $\begin{array}{c}2.956 \\
84.201 \%\end{array}$ & $\begin{array}{c}2.439 \\
82.502 \%\end{array}$ & $\begin{array}{c}2.005 \\
82.205 \%\end{array}$ & $\begin{array}{c}1.448 \\
72.232 \%\end{array}$ & $\begin{array}{c}0.880 \\
60.756 \%\end{array}$ & $1.677 \%$ \\
\hline & Ks pi pi0 & 22.780 & $\begin{array}{c}21.993 \\
96.548 \%\end{array}$ & $\begin{array}{c}0.426 \\
1.935 \%\end{array}$ & $\begin{array}{c}0.396 \\
93.030 \%\end{array}$ & $\begin{array}{c}0.286 \\
72.318 \%\end{array}$ & $\begin{array}{c}0.189 \\
66.003 \%\end{array}$ & $\begin{array}{c}0.141 \\
74.343 \%\end{array}$ & $\begin{array}{c}0.104 \\
74.354 \%\end{array}$ & $\begin{array}{c}0.045 \\
42.679 \%\end{array}$ & $0.196 \%$ \\
\hline & Ks pi pi & 16.708 & $\begin{array}{c}15.877 \\
95.030 \%\end{array}$ & $\begin{array}{c}0.679 \\
4.277 \%\end{array}$ & $\begin{array}{c}0.607 \\
89.354 \%\end{array}$ & $\begin{array}{c}0.465 \\
76.606 \%\end{array}$ & $\begin{array}{c}0.324 \\
69.681 \%\end{array}$ & $\begin{array}{c}0.249 \\
76.794 \%\end{array}$ & $\begin{array}{c}0.197 \\
79.330 \%\end{array}$ & $\begin{array}{c}0.086 \\
43.661 \%\end{array}$ & $0.516 \%$ \\
\hline & Ks pi pi pi0 & 12.245 & $\begin{array}{c}11.494 \\
93.867 \%\end{array}$ & $\begin{array}{c}0.080 \\
0.700 \%\end{array}$ & $\begin{array}{c}0.080 \\
100.000 \%\end{array}$ & $\begin{array}{c}0.055 \\
68.644 \%\end{array}$ & $\begin{array}{c}0.044 \\
79.386 \%\end{array}$ & $\begin{array}{c}0.028 \\
64.850 \%\end{array}$ & $\begin{array}{c}0.028 \\
100.000 \%\end{array}$ & $\begin{array}{c}0.000 \\
0.000 \%\end{array}$ & $0.000 \%$ \\
\hline & Ks pi pi pi & 4.569 & $\begin{array}{c}4.224 \\
92.448 \%\end{array}$ & $\begin{array}{c}0.074 \\
1.754 \%\end{array}$ & $\begin{array}{c}0.074 \\
100.000 \%\end{array}$ & $\begin{array}{c}0.052 \\
70.613 \%\end{array}$ & $\begin{array}{c}0.026 \\
49.137 \%\end{array}$ & $\begin{array}{c}0.020 \\
78.937 \%\end{array}$ & $\begin{array}{c}0.020 \\
100.000 \%\end{array}$ & $\begin{array}{c}0.000 \\
0.000 \%\end{array}$ & $0.000 \%$ \\
\hline & Total & 75.714 & $\begin{array}{c}718.177 \\
95.033 \%\end{array}$ & $\begin{array}{l}53.892 \\
7.504 \%\end{array}$ & $\begin{array}{c}32.397 \\
60.114 \%\end{array}$ & $\begin{array}{c}27.457 \\
84.752 \%\end{array}$ & $\begin{array}{c}23.339 \\
85.001 \%\end{array}$ & $\begin{array}{c}18.819 \\
80.636 \%\end{array}$ & $\begin{array}{c}13.789 \\
73.271 \%\end{array}$ & $\begin{array}{c}8.846 \\
64.150 \%\end{array}$ & $1.171 \%$ \\
\hline
\end{tabular}




\section{A.1 Background rejection tables}

In this section we list the background suppression rates for different stages of the analysis, as estimated in the MC. The table entries are in the $m_{\mathrm{ES}}$ range $5.20<m_{\mathrm{ES}}<5.29 \mathrm{GeV}$. The numbers correspond to number of events in 81.9

$\mathrm{fb}^{-1}$. The entries in [\%] show the efficiency of the corresponding step. The last column lists the overall efficiencies. 


\begin{tabular}{|c|c|c|c|c|c|c|}
\hline Topol & best Combo & preSel & postSel & jpsi veto & LR cut & tot Eff \\
\hline other & 0.00 & $\begin{array}{l}0.00 \\
0.0 \%\end{array}$ & $\begin{array}{l}0.00 \\
0.0 \%\end{array}$ & $\begin{array}{l}0.00 \\
0.0 \%\end{array}$ & $\begin{array}{c}0.00 \\
0.0 \%\end{array}$ & $0.0 \%$ \\
\hline K & 3391.32 & $\begin{array}{l}3391.32 \\
100.0 \%\end{array}$ & $\begin{array}{c}2463.31 \\
72.6 \%\end{array}$ & $\begin{array}{l}247.13 \\
10.0 \%\end{array}$ & $\begin{array}{l}22.86 \\
9.3 \%\end{array}$ & $0.7 \%$ \\
\hline $\mathrm{K}$ pi0 & 3929.69 & $\begin{array}{l}3929.69 \\
100.0 \%\end{array}$ & $\begin{array}{c}1996.85 \\
50.8 \%\end{array}$ & $\begin{array}{l}622.57 \\
31.2 \%\end{array}$ & $\begin{array}{l}42.32 \\
6.8 \%\end{array}$ & $1.1 \%$ \\
\hline K pi & 9057.81 & $\begin{array}{l}9057.81 \\
100.0 \%\end{array}$ & $\begin{array}{c}4941.15 \\
54.6 \%\end{array}$ & $\begin{array}{c}1341.56 \\
27.2 \%\end{array}$ & $\begin{array}{l}70.97 \\
5.3 \%\end{array}$ & $0.8 \%$ \\
\hline K pi pi0 & 7796.38 & $\begin{array}{l}7796.38 \\
100.0 \%\end{array}$ & $\begin{array}{c}2852.11 \\
36.6 \%\end{array}$ & $\begin{array}{c}1099.09 \\
38.5 \%\end{array}$ & $\begin{array}{l}35.45 \\
3.2 \%\end{array}$ & $0.5 \%$ \\
\hline K pi pi & 13523.41 & $\begin{array}{c}13523.41 \\
100.0 \%\end{array}$ & $\begin{array}{c}5350.83 \\
39.6 \%\end{array}$ & $\begin{array}{c}1853.75 \\
34.6 \%\end{array}$ & $\begin{array}{l}69.80 \\
3.8 \%\end{array}$ & $0.5 \%$ \\
\hline K pi pi pi0 & 6678.81 & $\begin{array}{l}6678.81 \\
100.0 \%\end{array}$ & $\begin{array}{c}1223.63 \\
18.3 \%\end{array}$ & $\begin{array}{l}566.38 \\
46.3 \%\end{array}$ & $\begin{array}{l}0.00 \\
0.0 \%\end{array}$ & $0.0 \%$ \\
\hline K pi pi pi & 7171.13 & $\begin{array}{l}7171.13 \\
100.0 \%\end{array}$ & $\begin{array}{c}1525.27 \\
21.3 \%\end{array}$ & $\begin{array}{l}682.33 \\
44.7 \%\end{array}$ & $\begin{array}{l}0.00 \\
0.0 \%\end{array}$ & $0.0 \%$ \\
\hline Ks & 1037.72 & $\begin{array}{l}1037.72 \\
100.0 \%\end{array}$ & $\begin{array}{l}730.58 \\
70.4 \%\end{array}$ & $\begin{array}{c}76.91 \\
10.5 \%\end{array}$ & $\begin{array}{l}4.95 \\
6.4 \%\end{array}$ & $0.5 \%$ \\
\hline Ks pi0 & 1105.39 & $\begin{array}{l}1105.39 \\
100.0 \%\end{array}$ & $\begin{array}{l}514.17 \\
46.5 \%\end{array}$ & $\begin{array}{l}167.16 \\
32.5 \%\end{array}$ & $\begin{array}{l}12.01 \\
7.2 \%\end{array}$ & $1.1 \%$ \\
\hline Ks pi & 3786.50 & $\begin{array}{l}3786.50 \\
100.0 \%\end{array}$ & $\begin{array}{c}1937.12 \\
51.2 \%\end{array}$ & $\begin{array}{l}577.60 \\
29.8 \%\end{array}$ & $\begin{array}{l}31.79 \\
5.5 \%\end{array}$ & $0.8 \%$ \\
\hline Ks pi pi0 & 3209.66 & $\begin{array}{l}3209.66 \\
100.0 \%\end{array}$ & $\begin{array}{c}1071.68 \\
33.4 \%\end{array}$ & $\begin{array}{l}401.65 \\
37.5 \%\end{array}$ & $\begin{array}{l}11.06 \\
2.8 \%\end{array}$ & $0.3 \%$ \\
\hline Ks pi pi & 3370.94 & $\begin{array}{l}3370.94 \\
100.0 \%\end{array}$ & $\begin{array}{c}1244.41 \\
36.9 \%\end{array}$ & $\begin{array}{l}442.52 \\
35.6 \%\end{array}$ & $\begin{array}{l}12.98 \\
2.9 \%\end{array}$ & $0.4 \%$ \\
\hline Ks pi pi pi0 & 1783.55 & $\begin{array}{l}1783.55 \\
100.0 \%\end{array}$ & $\begin{array}{l}312.21 \\
17.5 \%\end{array}$ & $\begin{array}{l}155.51 \\
49.8 \%\end{array}$ & $\begin{array}{l}0.00 \\
0.0 \%\end{array}$ & $0.0 \%$ \\
\hline Ks pi pi pi & 2710.58 & $\begin{array}{l}2710.58 \\
100.0 \%\end{array}$ & $\begin{array}{l}500.38 \\
18.5 \%\end{array}$ & $\begin{array}{l}235.09 \\
47.0 \%\end{array}$ & $\begin{array}{l}0.00 \\
0.0 \%\end{array}$ & $0.0 \%$ \\
\hline Total & 68552.88 & $\begin{array}{c}68552.88 \\
100.0 \%\end{array}$ & $\begin{array}{c}26663.70 \\
38.9 \%\end{array}$ & $\begin{array}{c}8469.24 \\
31.8 \%\end{array}$ & $\begin{array}{c}314.19 \\
3.7 \%\end{array}$ & $0.5 \%$ \\
\hline
\end{tabular}




\begin{tabular}{|c|c|c|c|c|c|c|}
\hline Topol & best Combo & preSel & postSel & jpsi veto & LR cut & tot Eff \\
\hline other & 0.00 & $\begin{array}{l}0.00 \\
0.0 \%\end{array}$ & $\begin{array}{l}0.00 \\
0.0 \%\end{array}$ & $\begin{array}{l}0.00 \\
0.0 \%\end{array}$ & $\begin{array}{l}0.00 \\
0.0 \%\end{array}$ & $0.0 \%$ \\
\hline $\mathrm{K}$ & 1430.27 & $\begin{array}{l}1430.27 \\
100.0 \%\end{array}$ & $\begin{array}{c}1185.85 \\
82.9 \%\end{array}$ & $\begin{array}{l}193.07 \\
16.3 \%\end{array}$ & $\begin{array}{c}30.69 \\
15.9 \%\end{array}$ & $2.1 \%$ \\
\hline K pi0 & 1314.68 & $\begin{array}{l}1314.68 \\
100.0 \%\end{array}$ & $\begin{array}{l}880.04 \\
66.9 \%\end{array}$ & $\begin{array}{l}333.57 \\
37.9 \%\end{array}$ & $\begin{array}{l}18.35 \\
5.5 \%\end{array}$ & $1.4 \%$ \\
\hline K pi & 3289.86 & $\begin{array}{l}3289.86 \\
100.0 \%\end{array}$ & $\begin{array}{c}2336.29 \\
71.0 \%\end{array}$ & $\begin{array}{l}775.51 \\
33.2 \%\end{array}$ & $\begin{array}{l}31.80 \\
4.1 \%\end{array}$ & $1.0 \%$ \\
\hline K pi pi0 & 2428.60 & $\begin{array}{l}2428.60 \\
100.0 \%\end{array}$ & $\begin{array}{c}1251.32 \\
51.5 \%\end{array}$ & $\begin{array}{l}496.38 \\
39.7 \%\end{array}$ & $\begin{array}{l}18.32 \\
3.7 \%\end{array}$ & $0.8 \%$ \\
\hline K pi pi & 4352.43 & $\begin{array}{l}4352.43 \\
100.0 \%\end{array}$ & $\begin{array}{c}2321.76 \\
53.3 \%\end{array}$ & $\begin{array}{l}919.33 \\
39.6 \%\end{array}$ & $\begin{array}{l}25.15 \\
2.7 \%\end{array}$ & $0.6 \%$ \\
\hline K pi pi pi0 & 1845.01 & $\begin{array}{l}1845.01 \\
100.0 \%\end{array}$ & $\begin{array}{l}502.26 \\
27.2 \%\end{array}$ & $\begin{array}{l}231.44 \\
46.1 \%\end{array}$ & $\begin{array}{l}0.00 \\
0.0 \%\end{array}$ & $0.0 \%$ \\
\hline K pi pi pi & 1992.88 & $\begin{array}{l}1992.88 \\
100.0 \%\end{array}$ & $\begin{array}{l}620.60 \\
31.1 \%\end{array}$ & $\begin{array}{l}261.02 \\
42.1 \%\end{array}$ & $\begin{array}{l}0.00 \\
0.0 \%\end{array}$ & $0.0 \%$ \\
\hline Ks & 417.80 & $\begin{array}{c}417.80 \\
100.0 \%\end{array}$ & $\begin{array}{l}350.80 \\
84.0 \%\end{array}$ & $\begin{array}{c}39.92 \\
11.4 \%\end{array}$ & $\begin{array}{c}8.92 \\
22.4 \%\end{array}$ & $2.1 \%$ \\
\hline Ks pi0 & 396.67 & $\begin{array}{c}396.67 \\
100.0 \%\end{array}$ & $\begin{array}{l}249.09 \\
62.8 \%\end{array}$ & $\begin{array}{c}99.27 \\
39.9 \%\end{array}$ & $\begin{array}{l}3.63 \\
3.7 \%\end{array}$ & $0.9 \%$ \\
\hline Ks pi & 1331.26 & $\begin{array}{l}1331.26 \\
100.0 \%\end{array}$ & $\begin{array}{l}837.06 \\
62.9 \%\end{array}$ & $\begin{array}{l}299.54 \\
35.8 \%\end{array}$ & $\begin{array}{l}14.44 \\
4.8 \%\end{array}$ & $1.1 \%$ \\
\hline Ks pi pi0 & 939.24 & $\begin{array}{c}939.24 \\
100.0 \%\end{array}$ & $\begin{array}{l}424.45 \\
45.2 \%\end{array}$ & $\begin{array}{l}177.16 \\
41.7 \%\end{array}$ & $\begin{array}{r}6.69 \\
3.8 \%\end{array}$ & $0.7 \%$ \\
\hline Ks pi pi & 1075.50 & $\begin{array}{l}1075.50 \\
100.0 \%\end{array}$ & $\begin{array}{l}562.19 \\
52.3 \%\end{array}$ & $\begin{array}{l}198.85 \\
35.4 \%\end{array}$ & $\begin{array}{l}4.56 \\
2.3 \%\end{array}$ & $0.4 \%$ \\
\hline Ks pi pi pi0 & 452.19 & $\begin{array}{l}452.19 \\
100.0 \%\end{array}$ & $\begin{array}{l}127.28 \\
28.1 \%\end{array}$ & $\begin{array}{c}60.04 \\
47.2 \%\end{array}$ & $\begin{array}{l}0.00 \\
0.0 \%\end{array}$ & $0.0 \%$ \\
\hline Ks pi pi pi & 747.28 & $\begin{array}{c}747.28 \\
100.0 \%\end{array}$ & $\begin{array}{l}197.39 \\
26.4 \%\end{array}$ & $\begin{array}{l}85.17 \\
43.1 \%\end{array}$ & $\begin{array}{l}0.00 \\
0.0 \%\end{array}$ & $0.0 \%$ \\
\hline Total & 22013.65 & $\begin{array}{c}22013.65 \\
100.0 \%\end{array}$ & $\begin{array}{c}11846.38 \\
53.8 \%\end{array}$ & $\begin{array}{l}4170.26 \\
35.2 \%\end{array}$ & $\begin{array}{c}162.54 \\
3.9 \%\end{array}$ & $0.7 \%$ \\
\hline
\end{tabular}


032796

\section{A P P E N D I X B}

\section{Background suppression variables}

Figures 78-94 show the background suppression variables used in the likelihood ratio, for $B \rightarrow X_{s} e^{+} e^{-}$and $B \rightarrow X_{s} \mu^{+} \mu^{-}$candidates in the four different categories of events: signal, cross-feed, $B \bar{B}$ and continuum MC. 

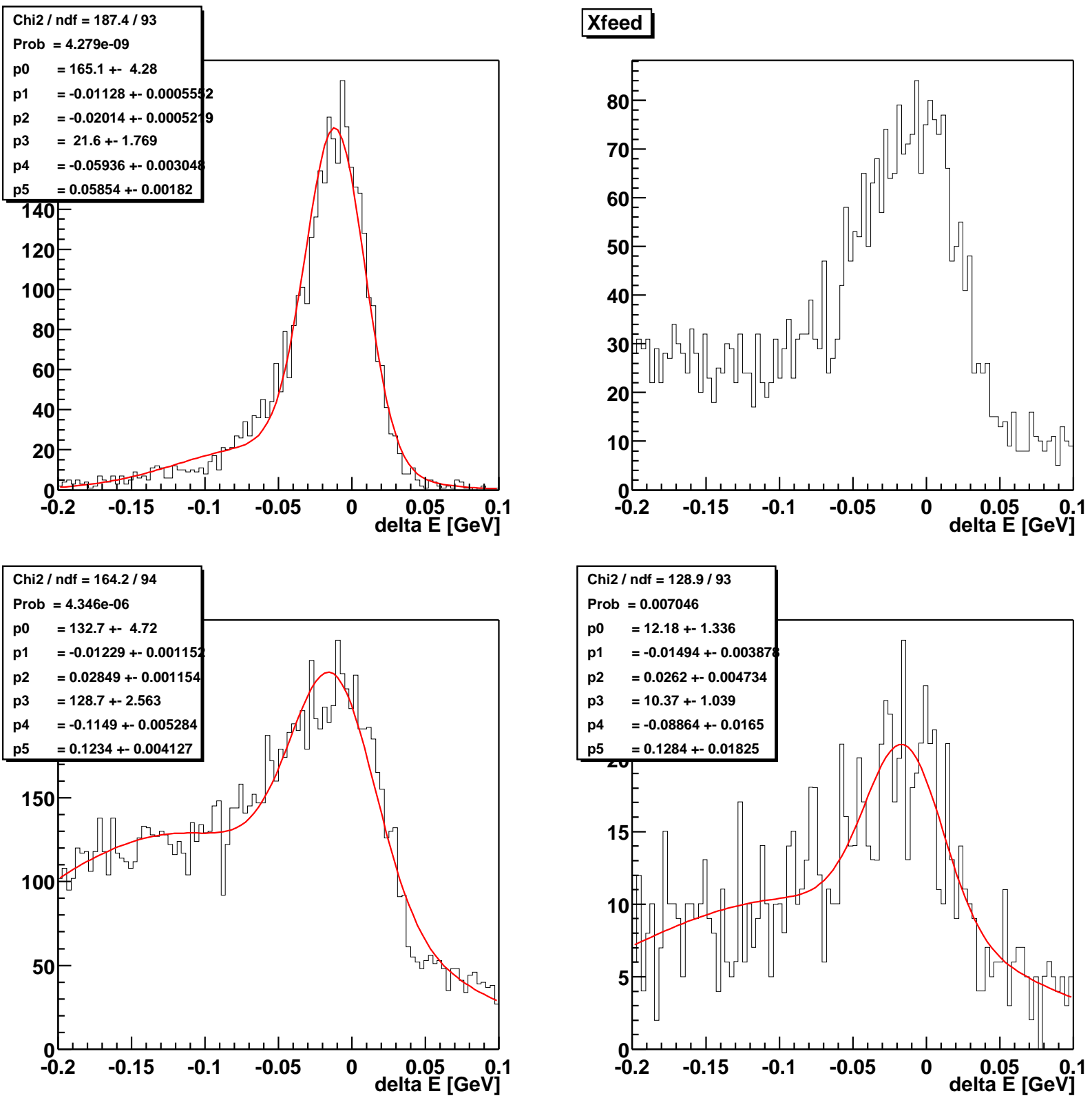

Figure 78: Distributions of $\Delta E$ for $B \rightarrow X_{s} e^{+} e^{-}$candidates in truth-matched signal (top left), cross-feed (top right), $B \bar{B}$ (bottom left) and continuum (bottom right) events. The curves show the result of the fits to these distributions using the functions listed in Table 5. 

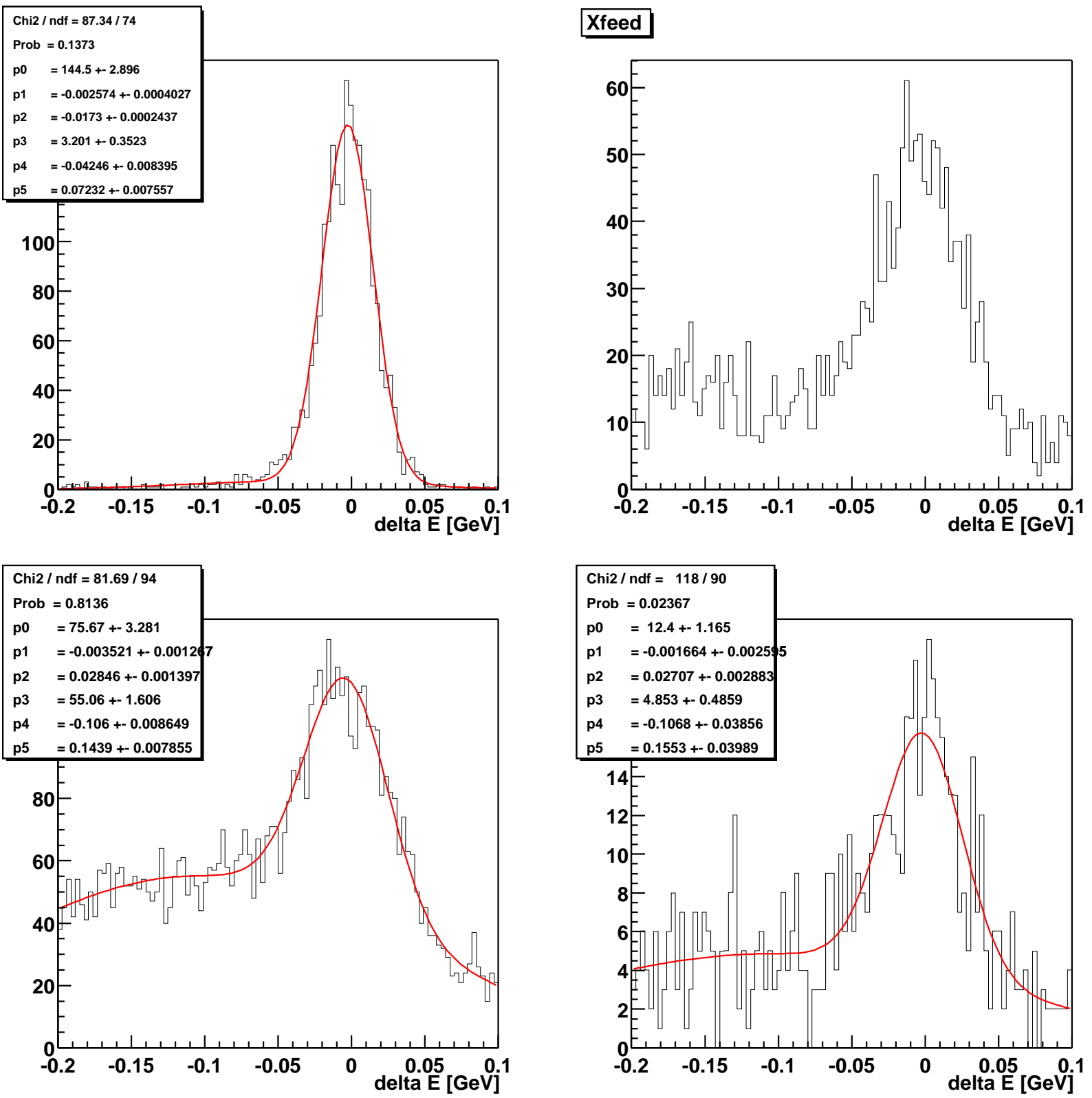

Figure 79: Distributions of $\Delta E$ for $B \rightarrow X_{s} \mu^{+} \mu^{-}$candidates in truth-matched signal (top left), cross-feed (top right), $B \bar{B}$ (bottom left) and continuum (bottom right) events. The curves show the result of the fits to these distributions using the functions listed in Table 5. 

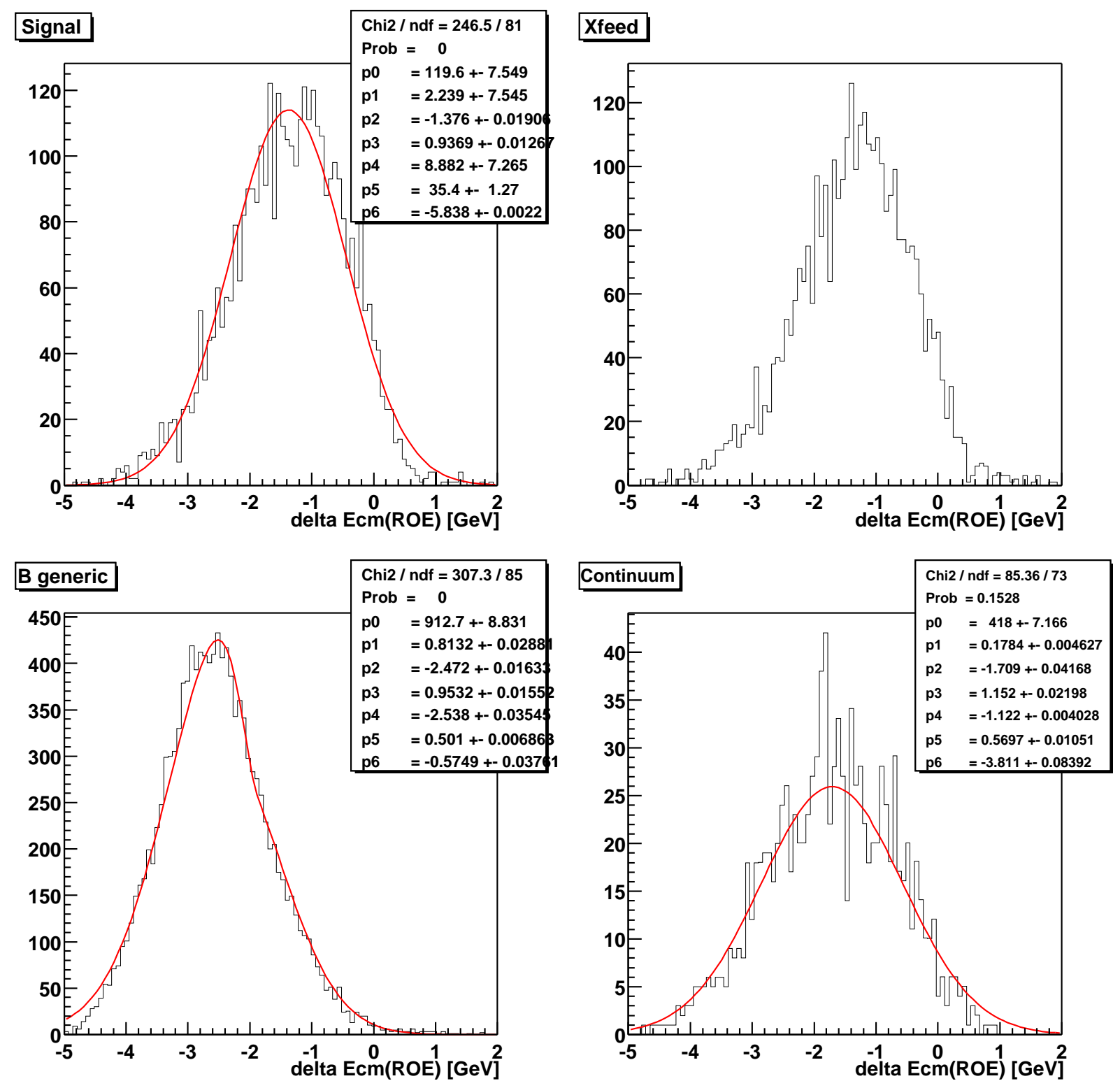

Figure 80: Distributions of $\Delta E^{R O E}$ for $B \rightarrow X_{s} e^{+} e^{-}$candidates in truthmatched signal (top left), cross-feed (top right), $B \bar{B}$ (bottom left) and continuum (bottom right) events. The curves show the result of the fits to these distributions using the functions listed in Table 5. 

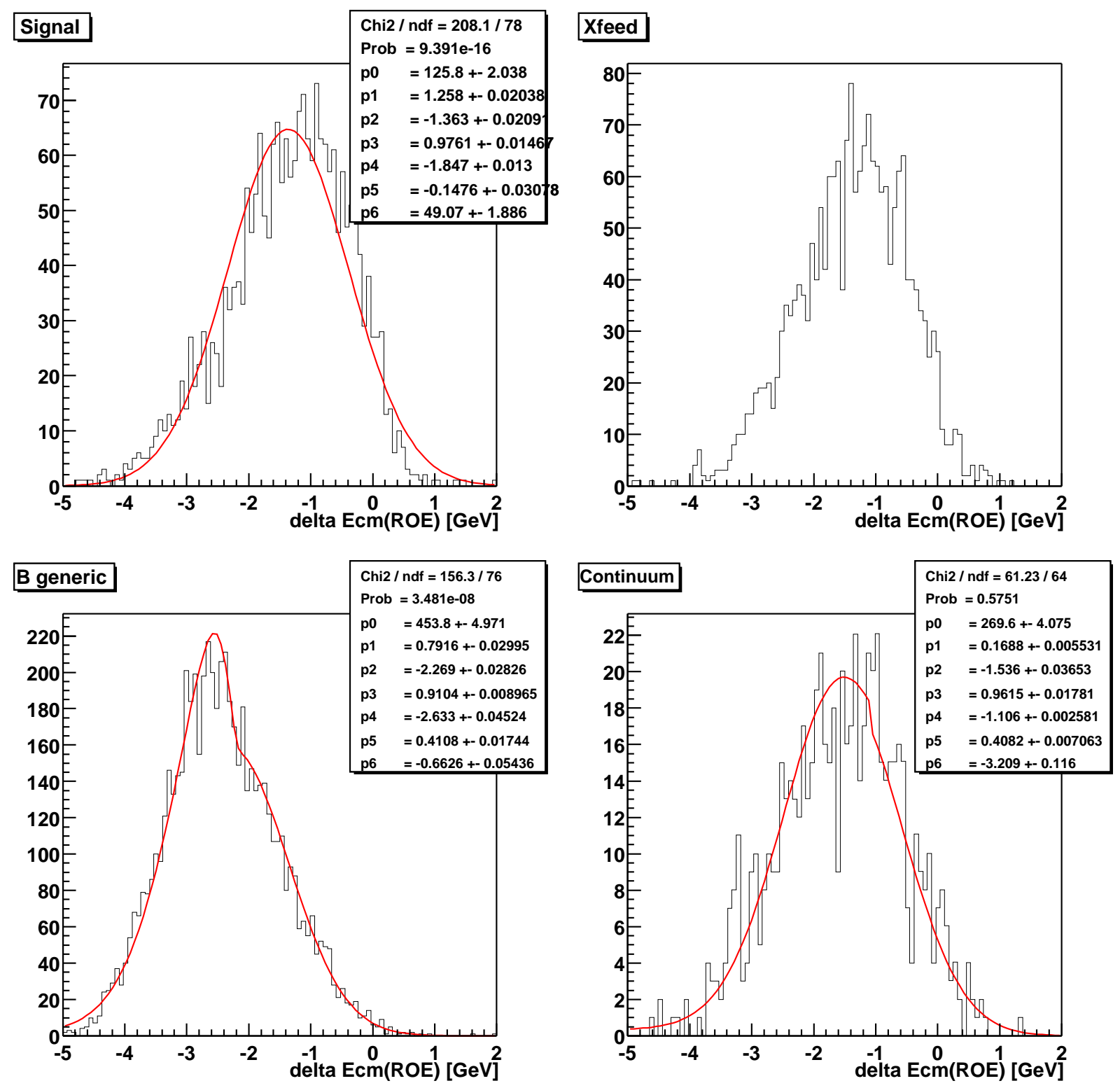

Figure 81: Distributions of $\Delta E^{R O E}$ for $B \rightarrow X_{s} \mu^{+} \mu^{-}$candidates in truthmatched signal (top left), cross-feed (top right), $B \bar{B}$ (bottom left) and continuum (bottom right) events. The curves show the result of the fits to these distributions using the functions listed in Table 5. 

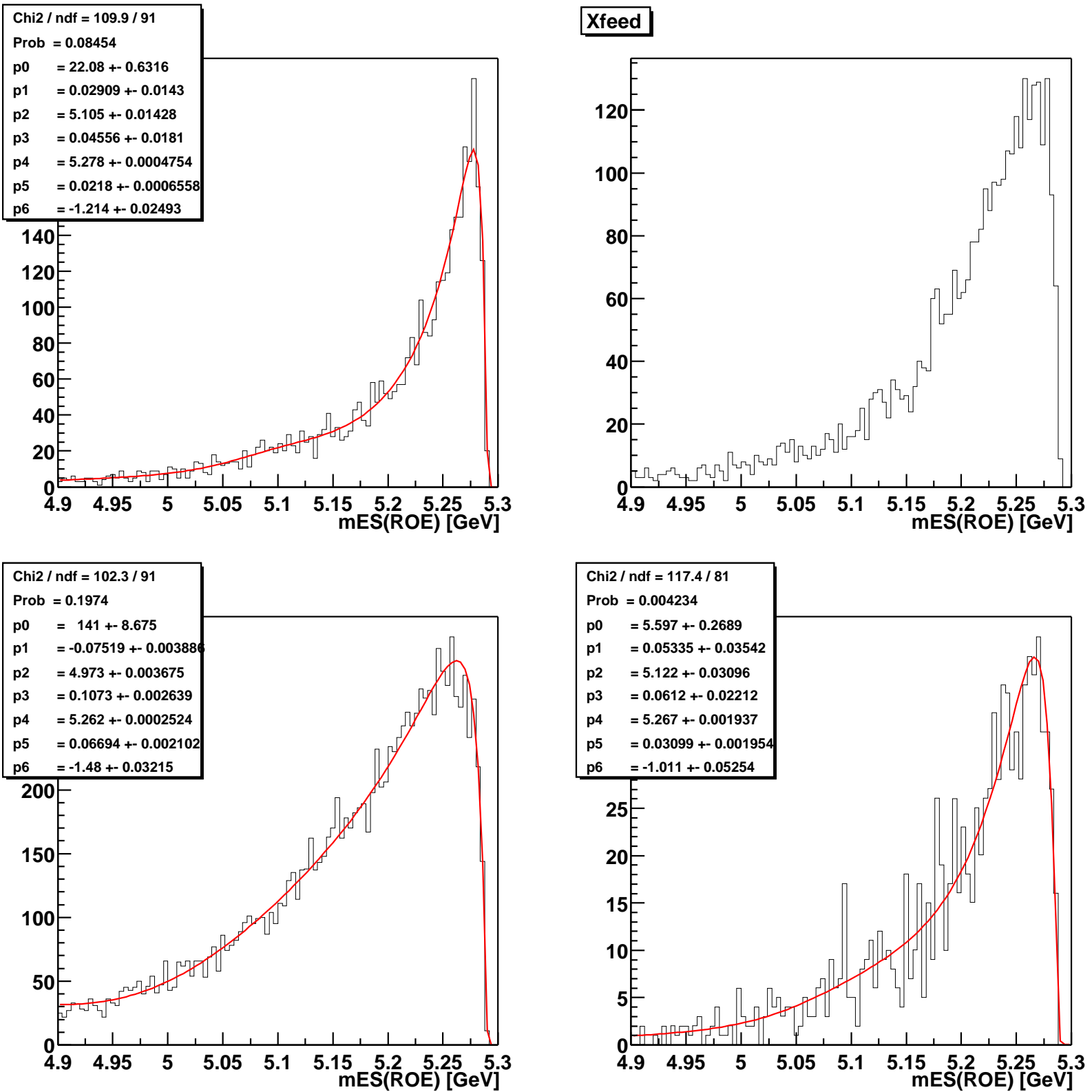

Figure 82: Distributions of $m_{E S}^{R O E}$ for $B \rightarrow X_{s} e^{+} e^{-}$candidates in truthmatched signal (top left), cross-feed (top right), $B \bar{B}$ (bottom left) and continuum (bottom right) events. The curves show the result of the fits to these distributions using the functions listed in Table 5. 

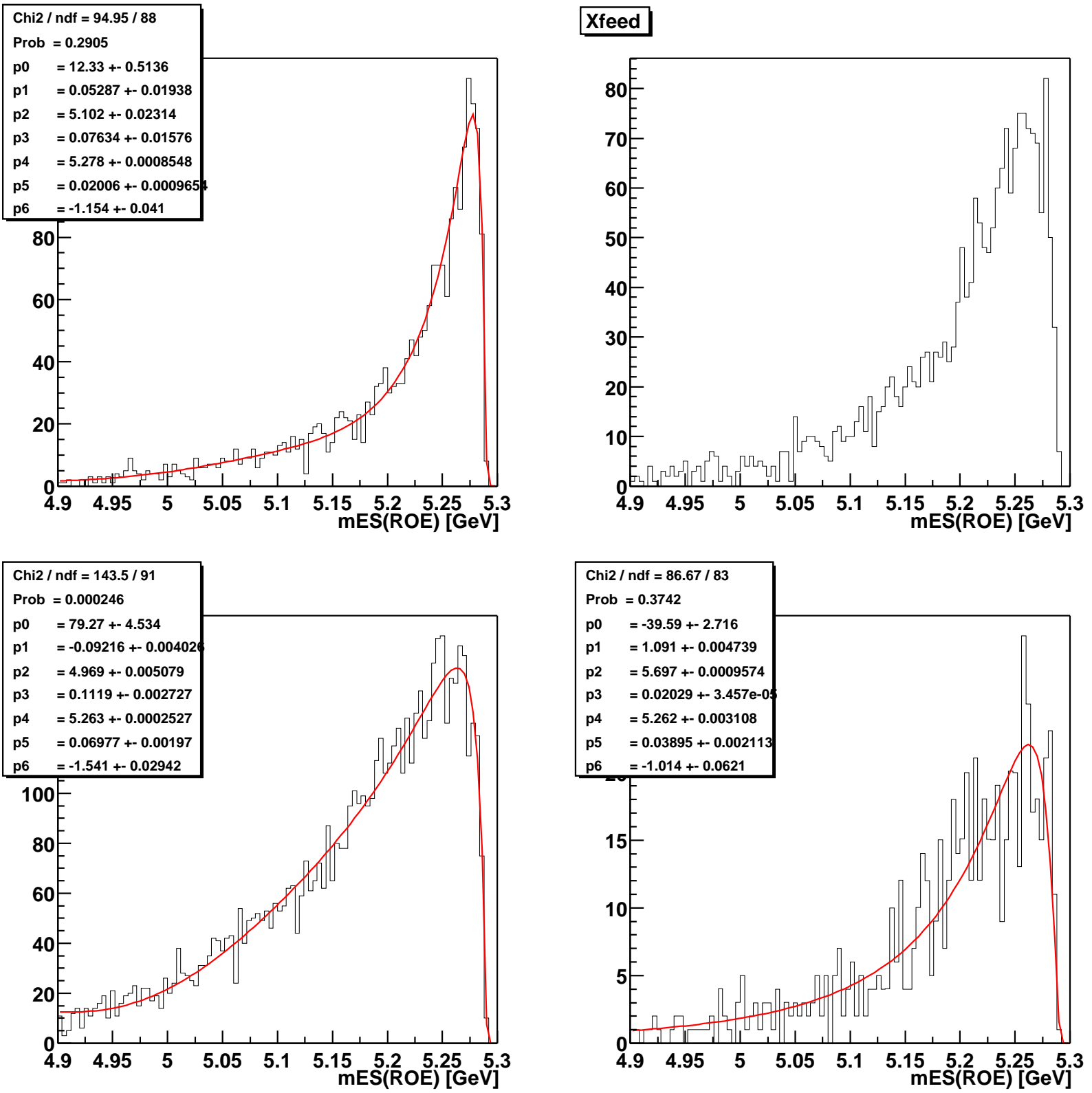

Figure 83: Distributions of $m_{E S}^{R O E}$ for $B \rightarrow X_{s} \mu^{+} \mu^{-}$candidates in truthmatched signal (top left), cross-feed (top right), $B \bar{B}$ (bottom left) and continuum (bottom right) events. The curves show the result of the fits to these distributions using the functions listed in Table 5. 


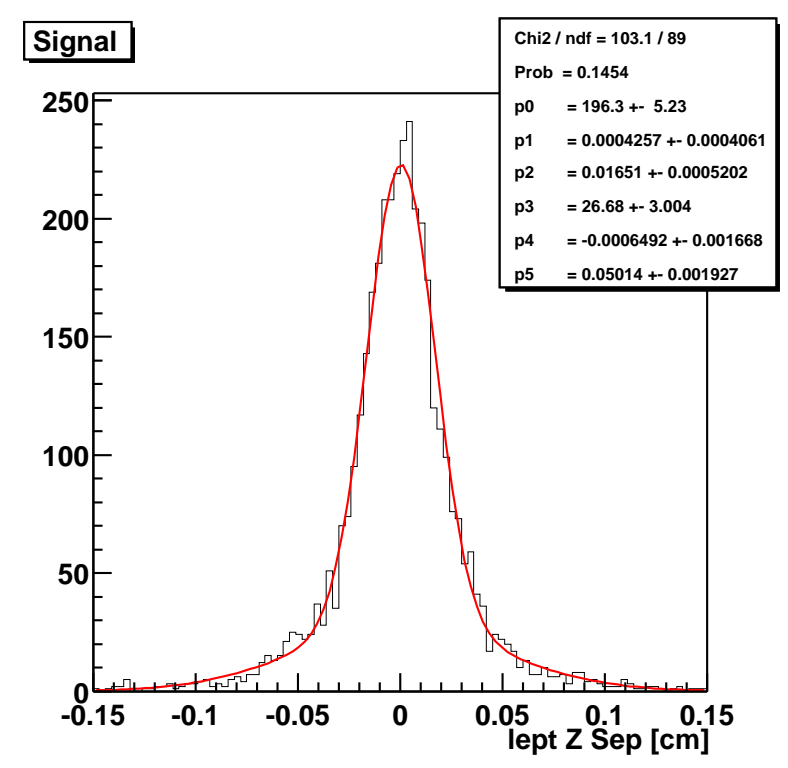

Xfeed
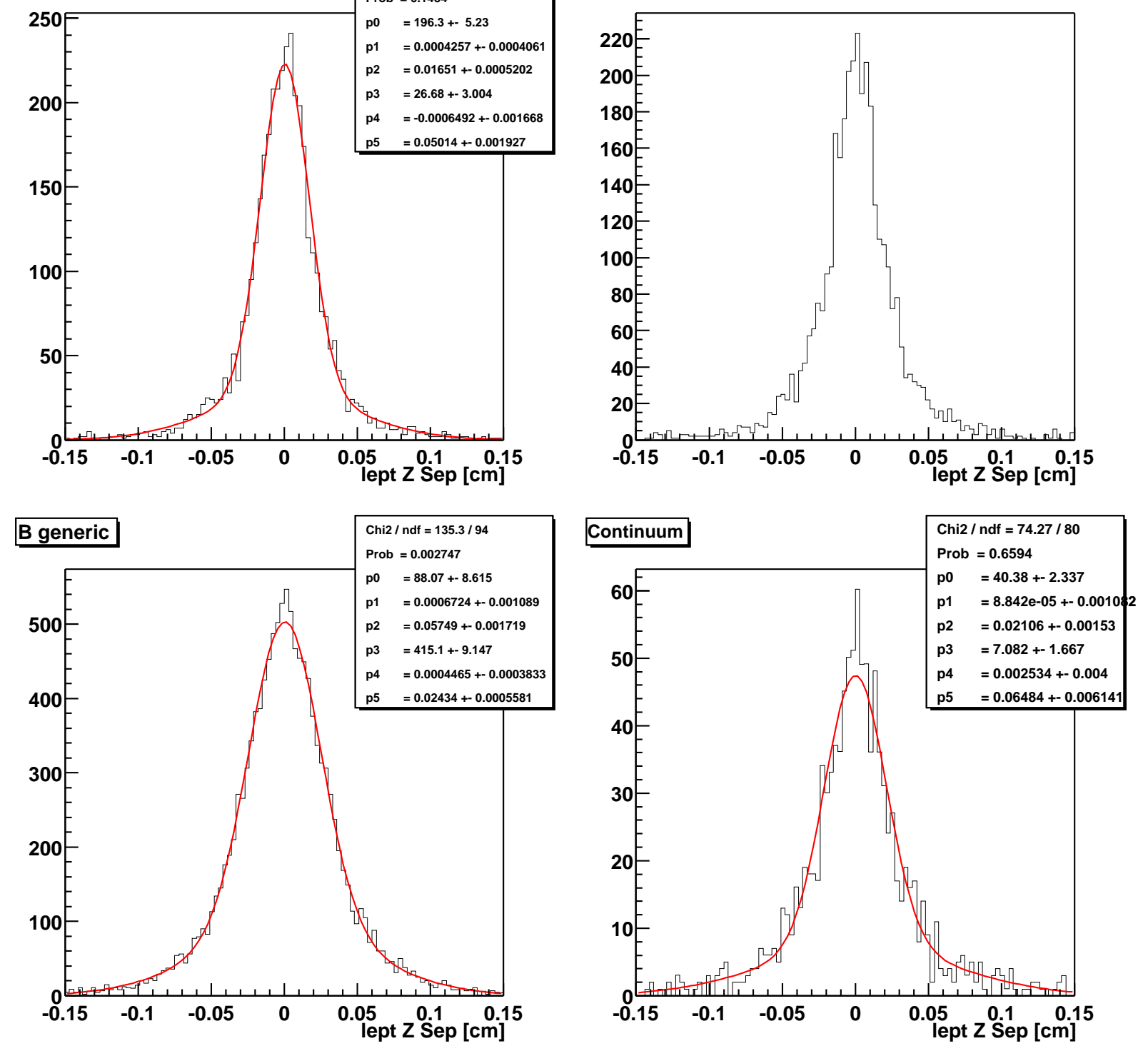

Figure 84: Distributions of $\Delta z$ between the leptons for $B \rightarrow X_{s} e^{+} e^{-}$candidates in truth-matched signal (top left), cross-feed (top right), $B \bar{B}$ (bottom left) and continuum (bottom right) events. The curves show the result of the fits to these distributions using the functions listed in Table 5. 


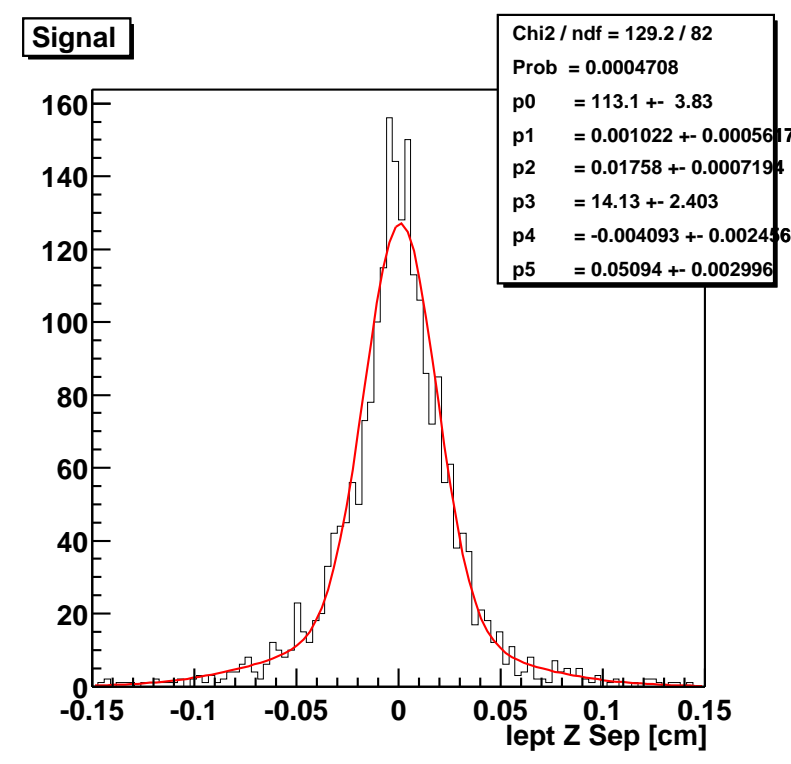

\section{Xfeed}
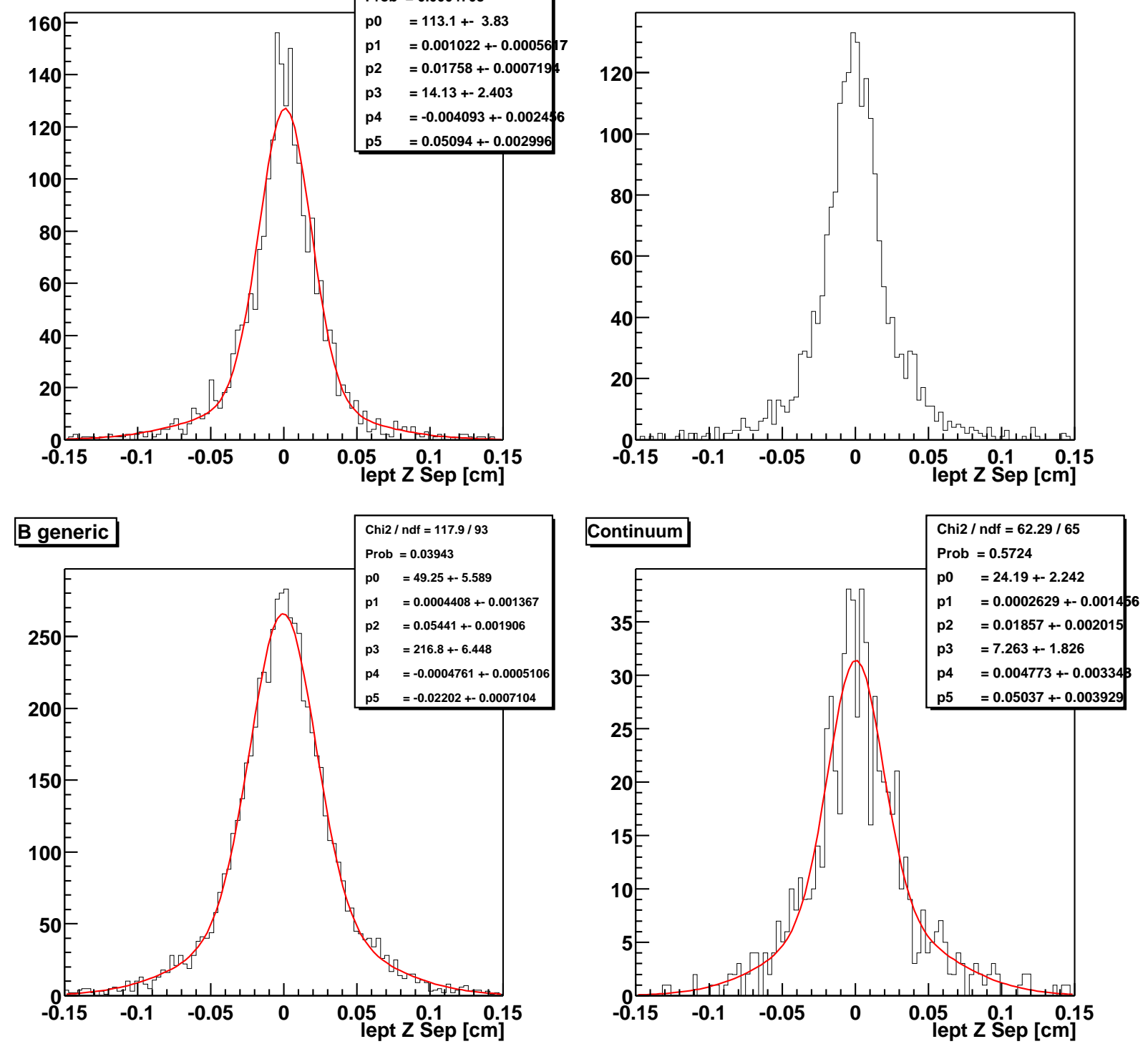

Figure 85: Distributions of $\Delta z$ between the leptons for $B \rightarrow X_{s} \mu^{+} \mu^{-}$candidates in truth-matched signal (top left), cross-feed (top right), $B \bar{B}$ (bottom left) and continuum (bottom right) events. The curves show the result of the fits to these distributions using the functions listed in Table 5 . 


\section{Signal}

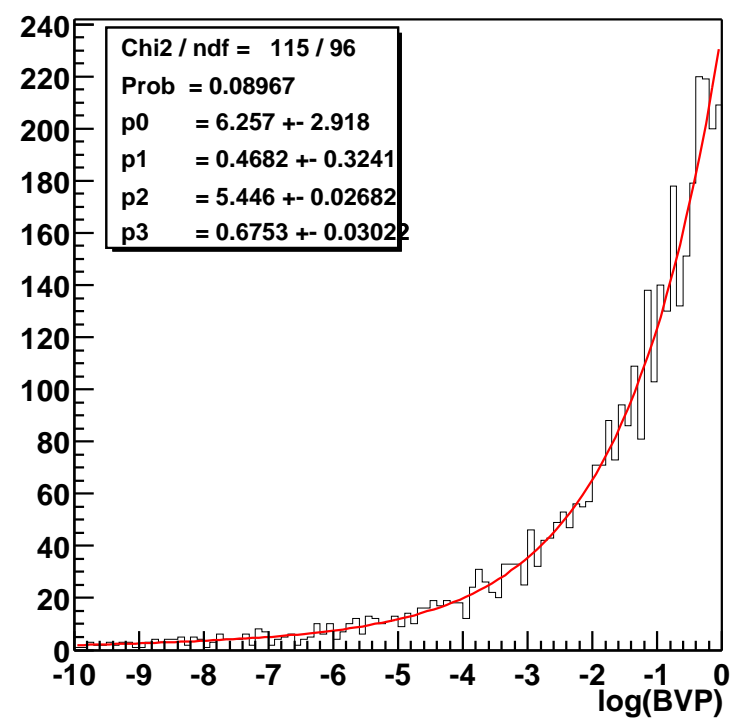

B generic

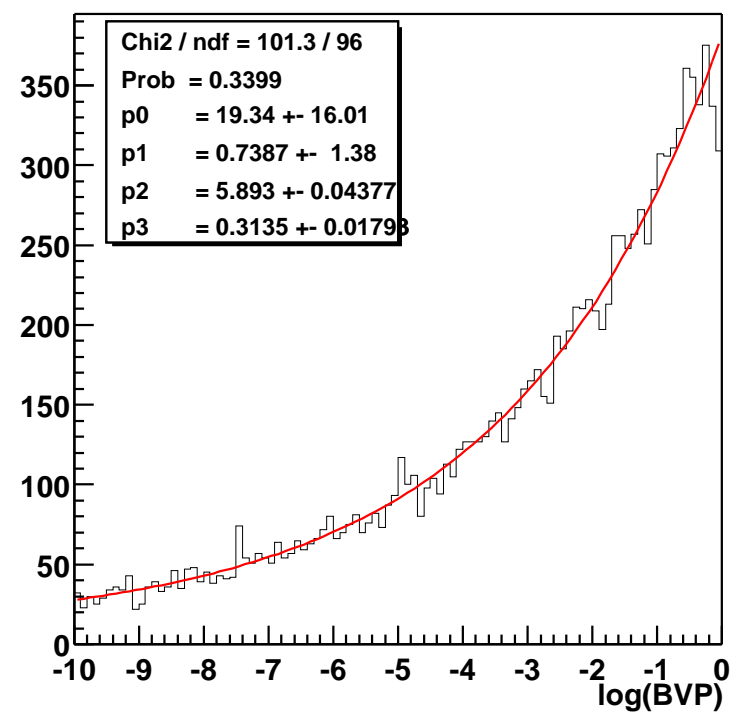

\section{Xfeed}

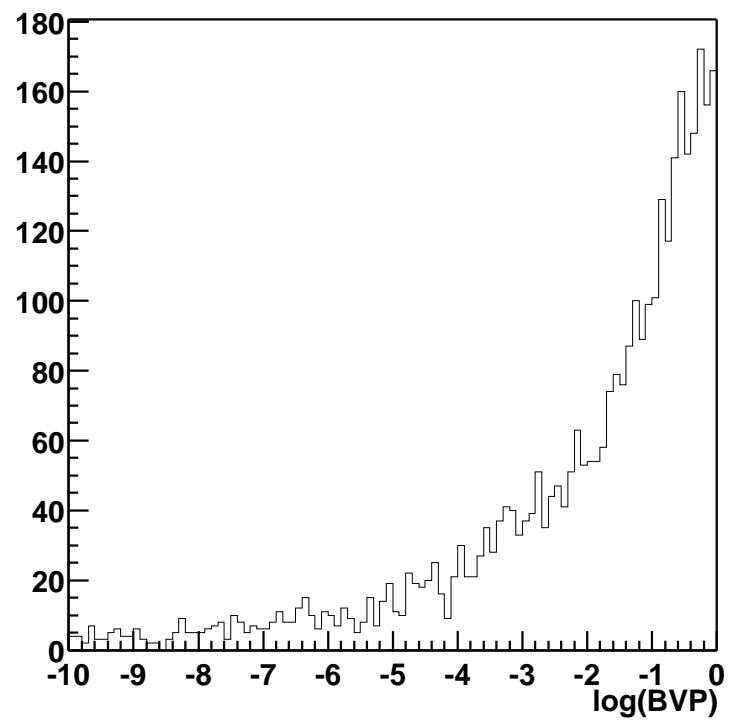

Continuum

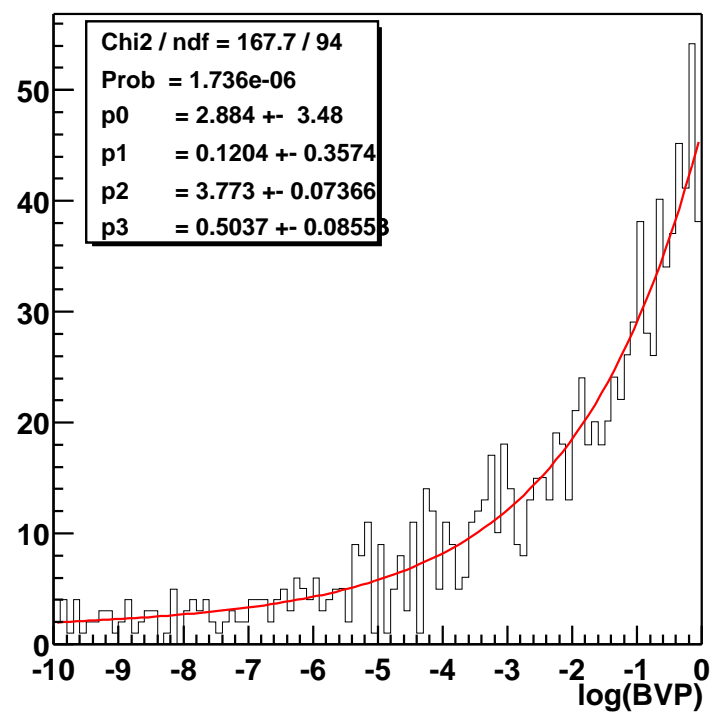

Figure 86: Distributions of $\log \left(P_{B v t x}\right)$ for $B \rightarrow X_{s} e^{+} e^{-}$candidates in truthmatched signal (top left), cross-feed (top right), $B \bar{B}$ (bottom left) and continuum (bottom right) events. The curves show the result of the fits to these distributions using the functions listed in Table 5. 
Signal

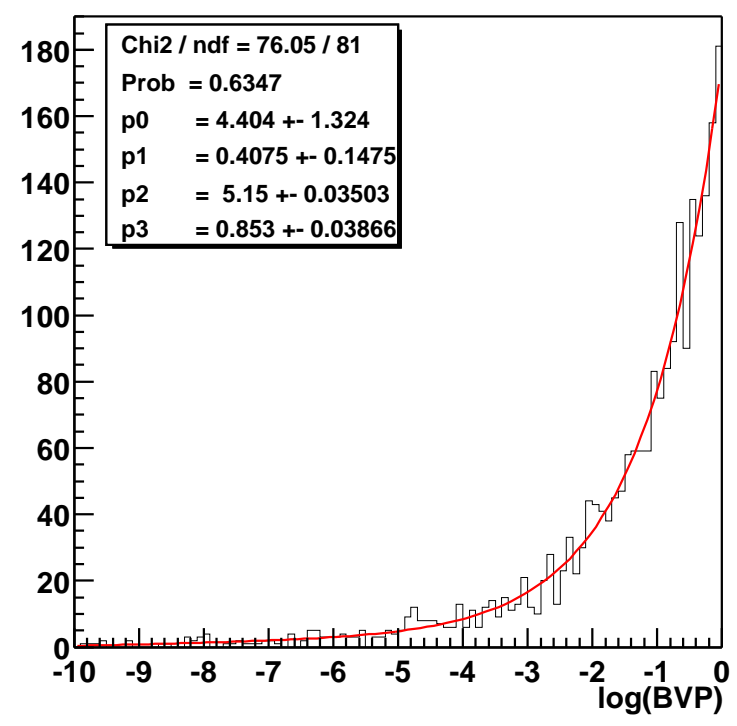

B generic

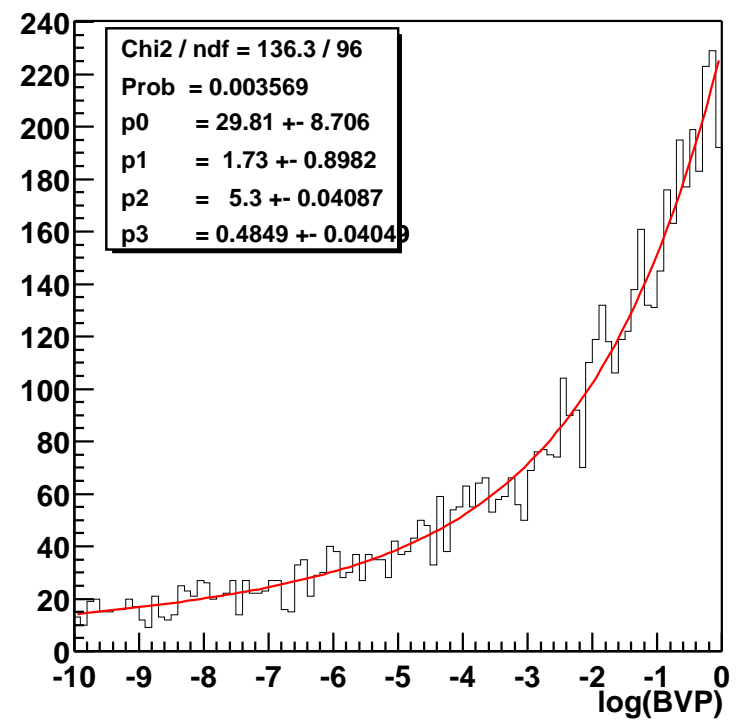

Xfeed

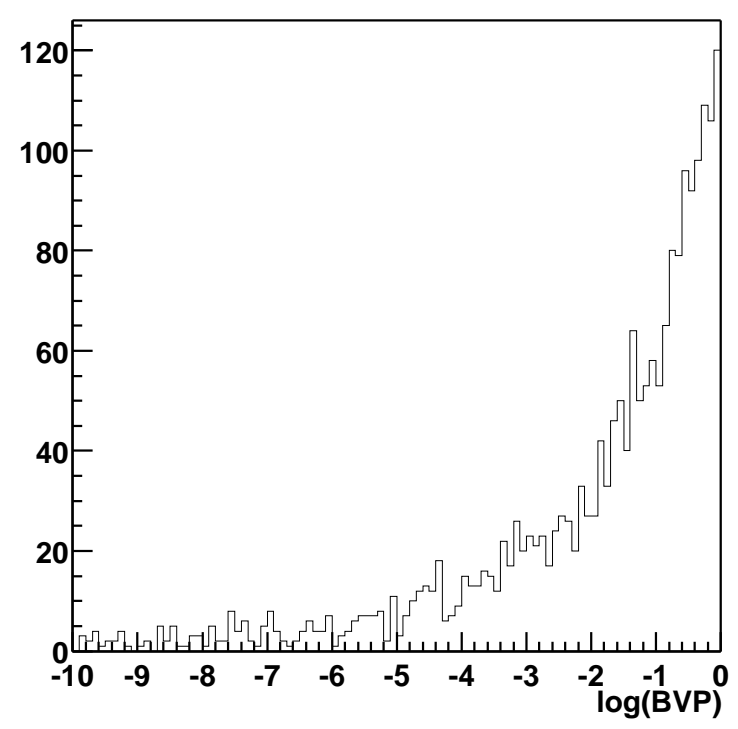

Continuum

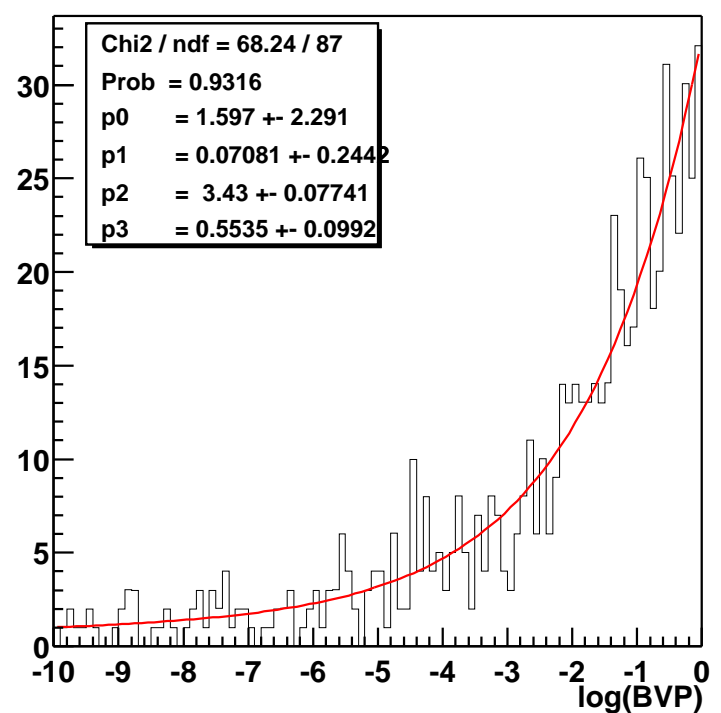

Figure 87: Distributions of $\log \left(P_{B v t x}\right)$ for $B \rightarrow X_{s} \mu^{+} \mu^{-}$candidates in truthmatched signal (top left), cross-feed (top right), $B \bar{B}$ (bottom left) and continuum (bottom right) events. The curves show the result of the fits to these distributions using the functions listed in Table 5. 

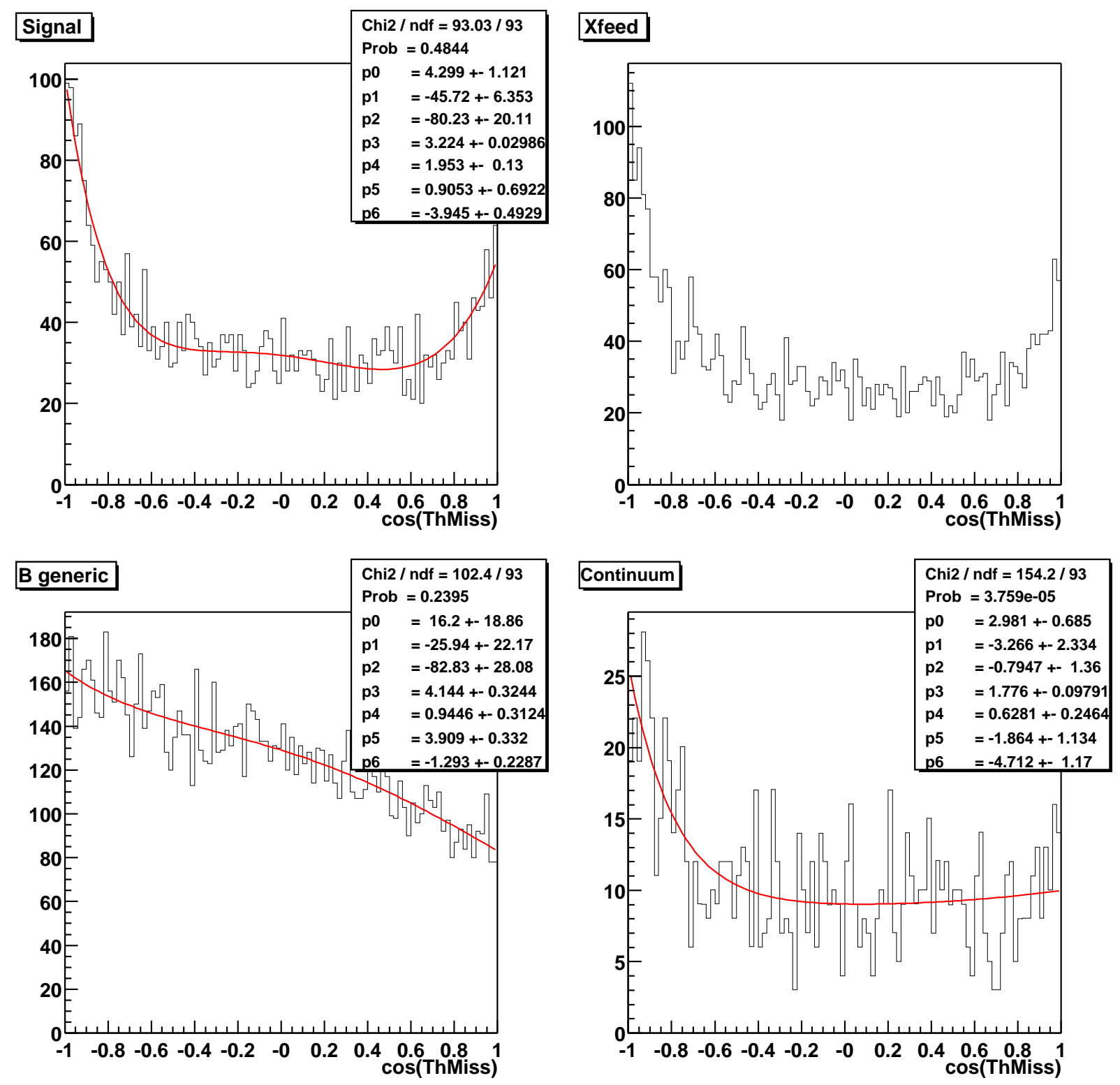

Figure 88: Distributions of $\cos \theta_{\text {miss }}$ for $B \rightarrow X_{s} e^{+} e^{-}$candidates in truthmatched signal (top left), cross-feed (top right), $B \bar{B}$ (bottom left) and continuum (bottom right) events. The curves show the result of the fits to these distributions using the functions listed in Table 5. 

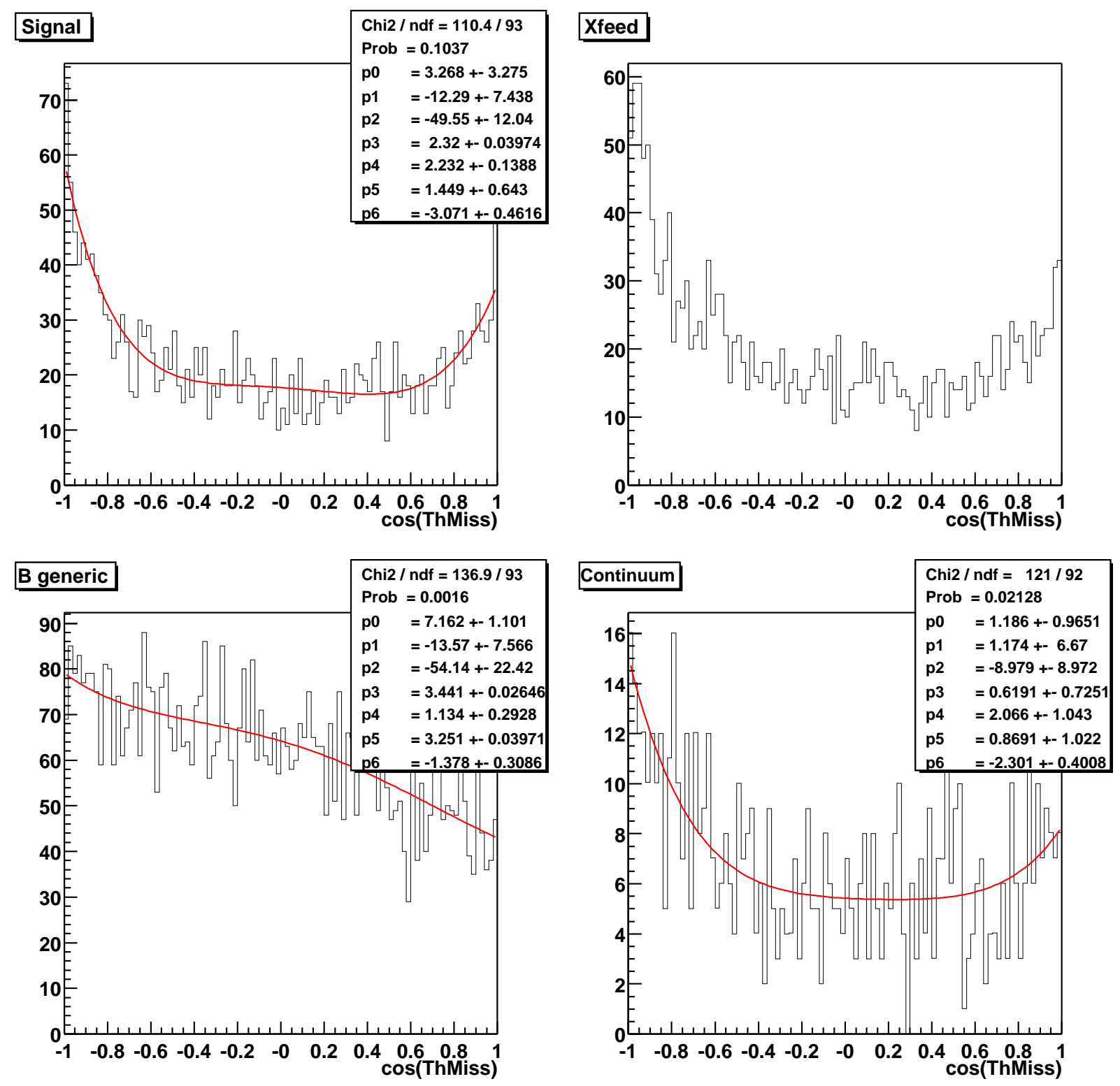

Figure 89: Distributions of $\cos \theta_{\text {miss }}$ for $B \rightarrow X_{s} \mu^{+} \mu^{-}$candidates in truthmatched signal (top left), cross-feed (top right), $B \bar{B}$ (bottom left) and continuum (bottom right) events. The curves show the result of the fits to these distributions using the functions listed in Table 5. 


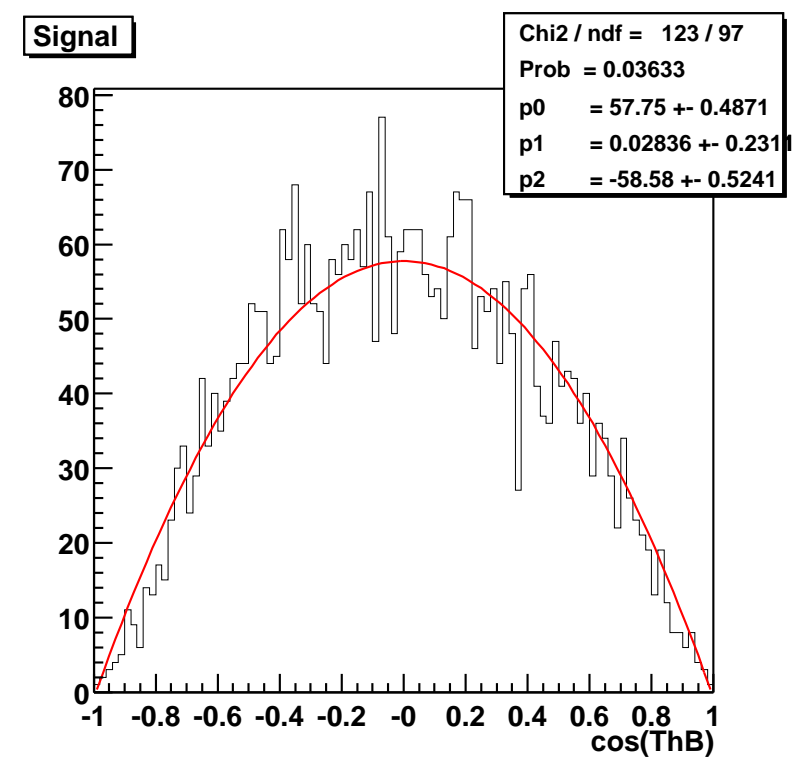

Xfeed
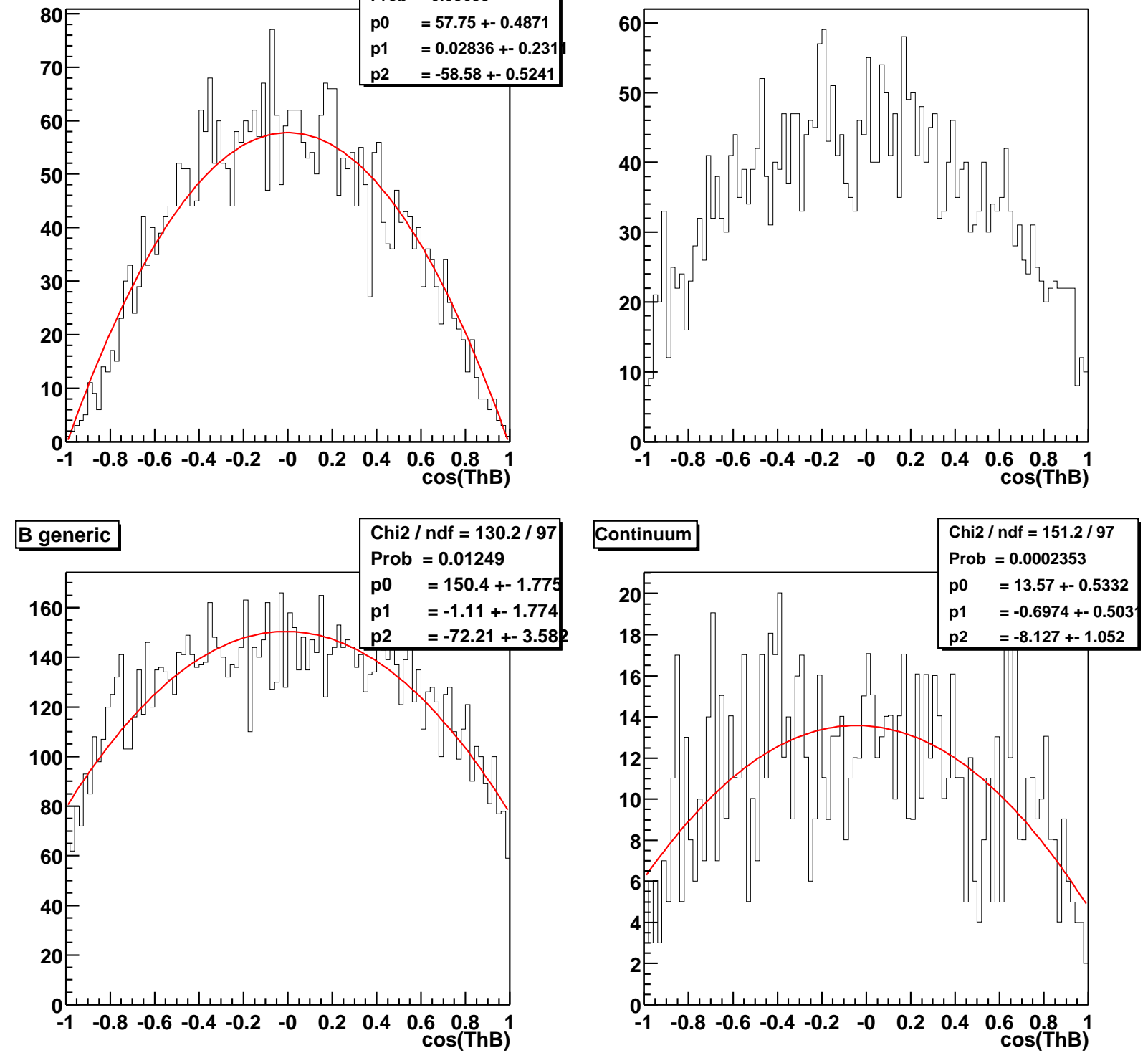

Figure 90: Distributions of $\cos \theta_{B}$ for $B \rightarrow X_{s} e^{+} e^{-}$candidates in truthmatched signal (top left), cross-feed (top right), $B \bar{B}$ (bottom left) and continuum (bottom right) events. The curves show the result of the fits to these distributions using the functions listed in Table 5. 


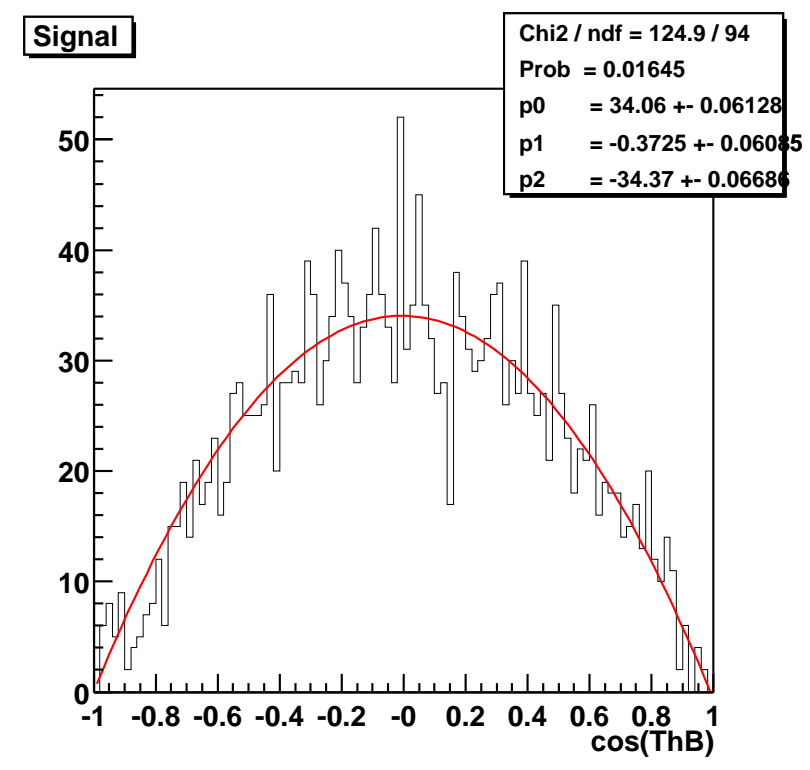

Xfeed
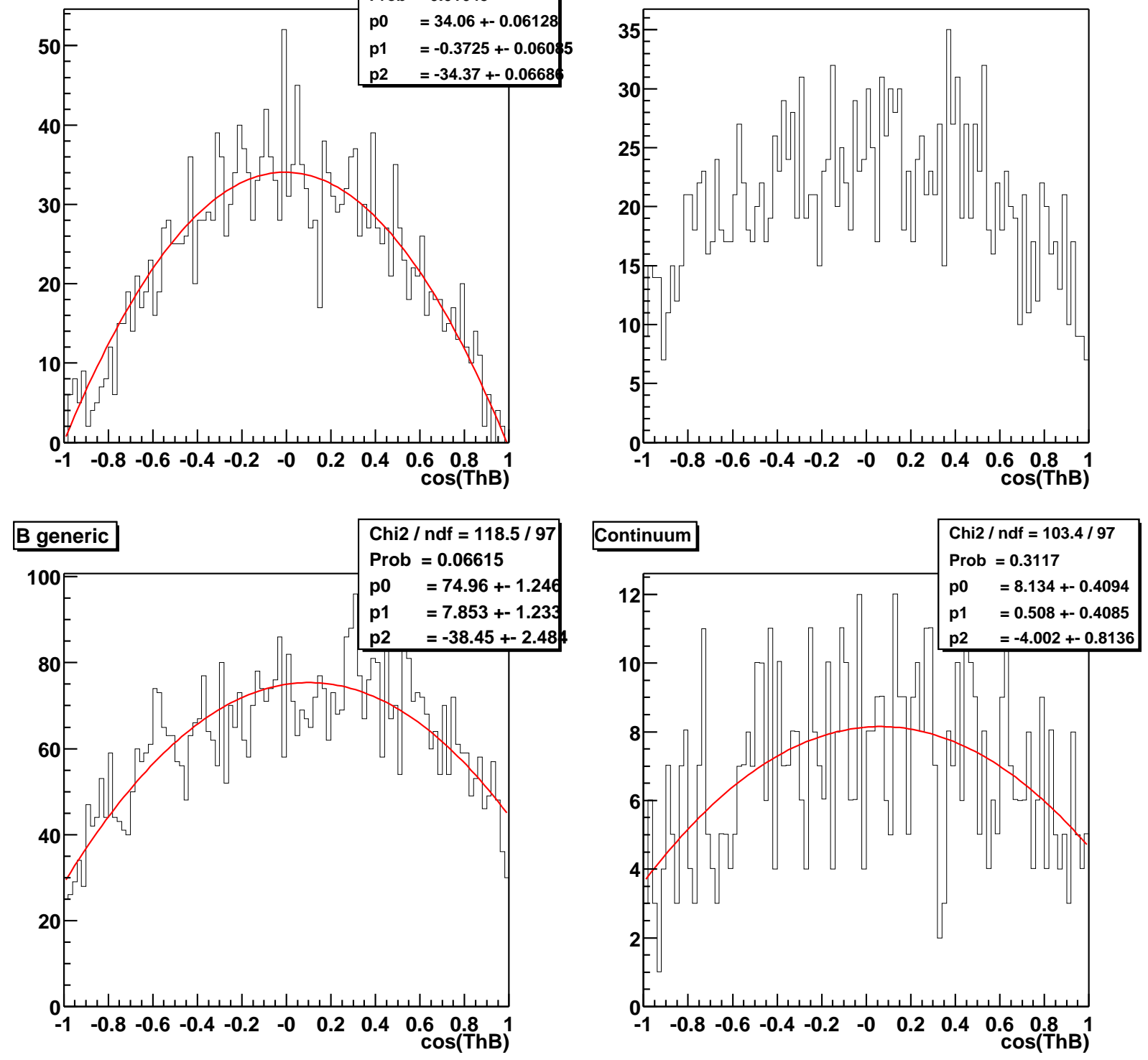

Figure 91: Distributions of $\cos \theta_{B}$ for $B \rightarrow X_{s} \mu^{+} \mu^{-}$candidates in truthmatched signal (top left), cross-feed (top right), $B \bar{B}$ (bottom left) and continuum (bottom right) events. The curves show the result of the fits to these distributions using the functions listed in Table 5. 
Signal

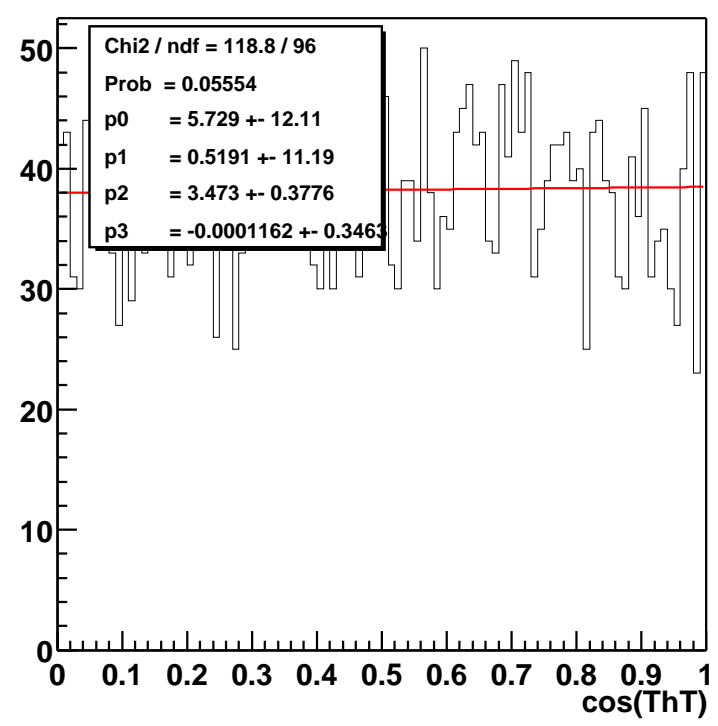

$B$ generic

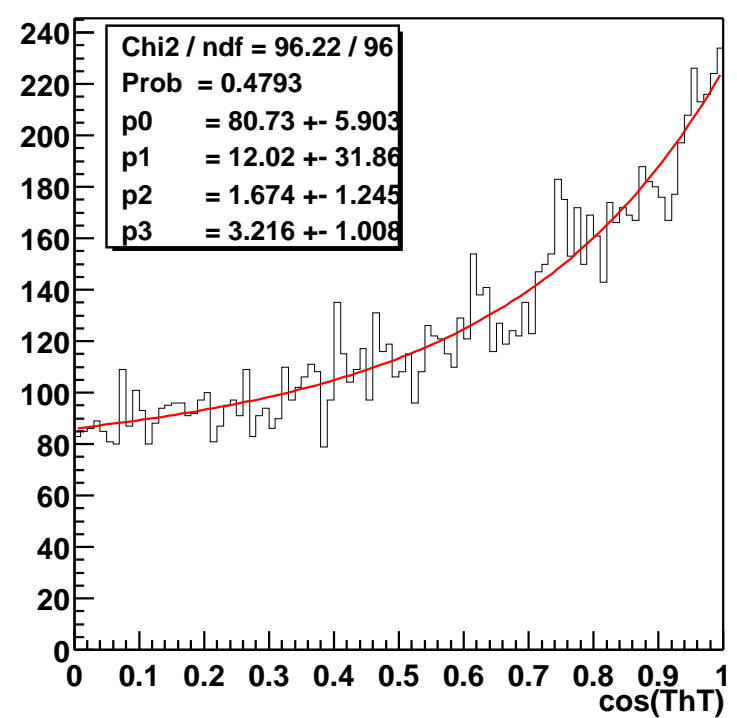

Xfeed

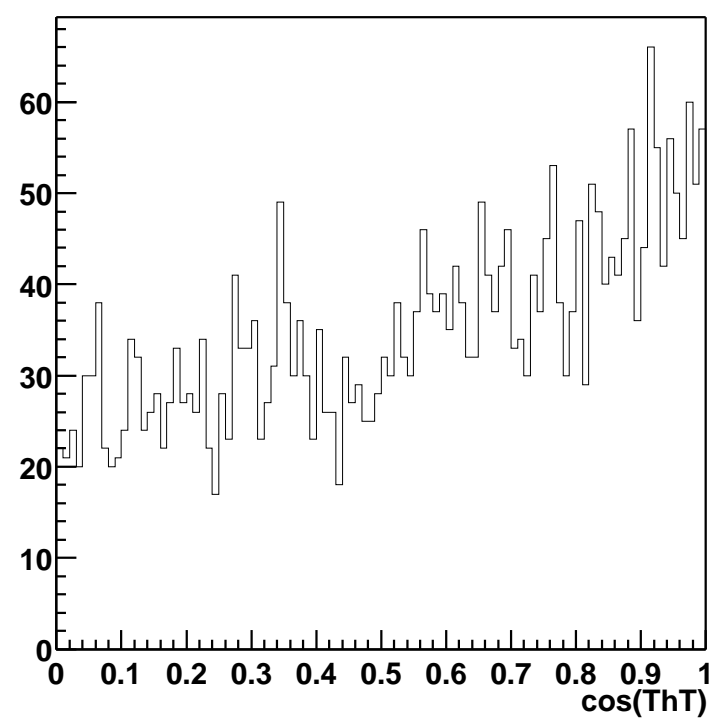

Continuum

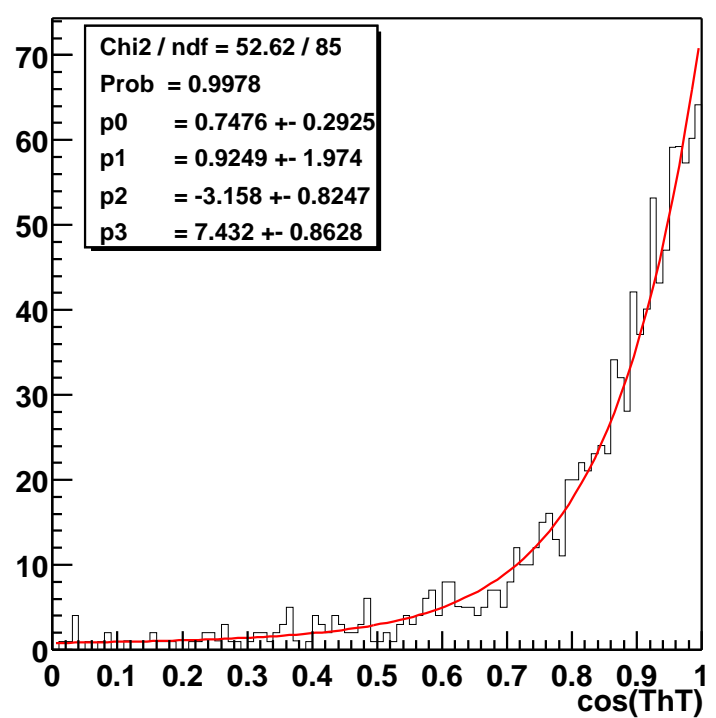

Figure 92: Distributions of $\left|\cos \theta_{T}\right|$ for $B \rightarrow X_{s} e^{+} e^{-}$candidates in truthmatched signal (top left), cross-feed (top right), $B \bar{B}$ (bottom left) and continuum (bottom right) events. The curves show the result of the fits to these distributions using the functions listed in Table 5. 
Signal

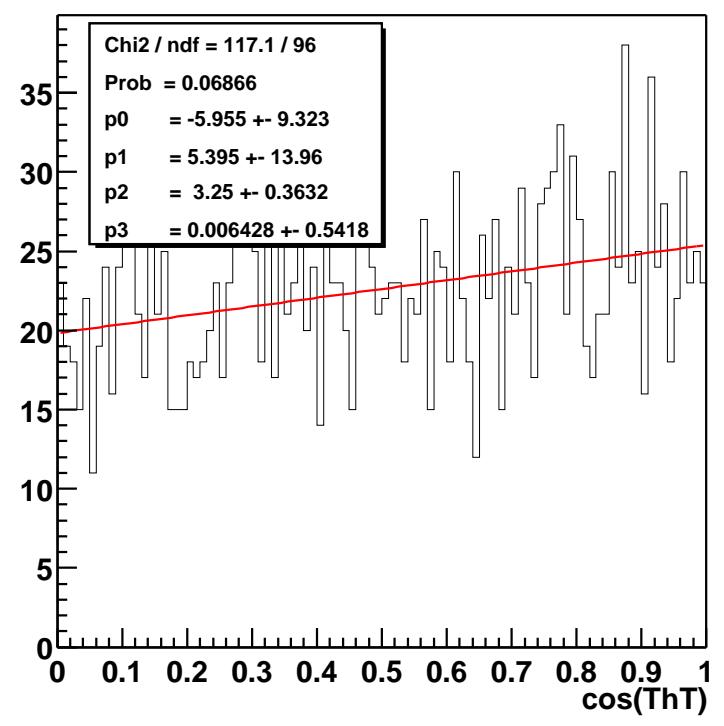

$B$ generic

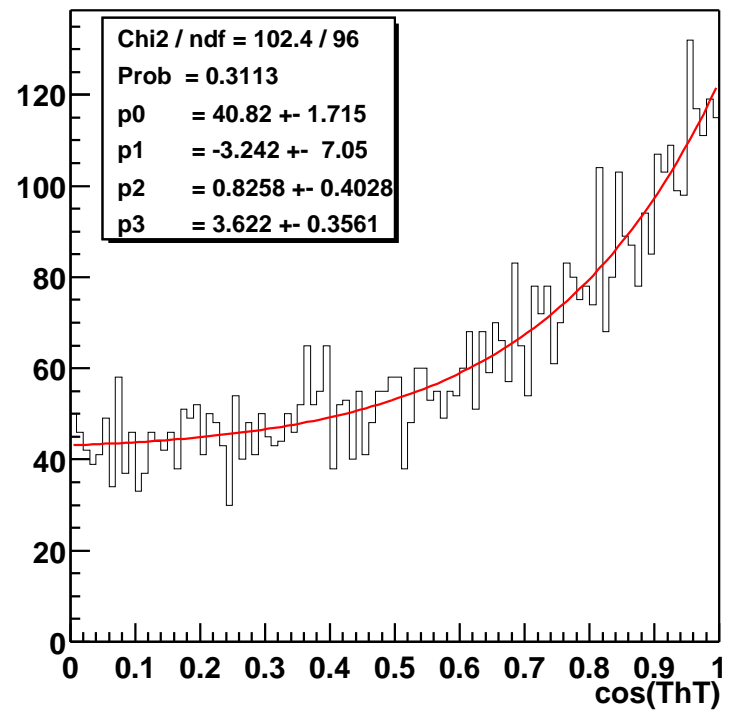

Xfeed

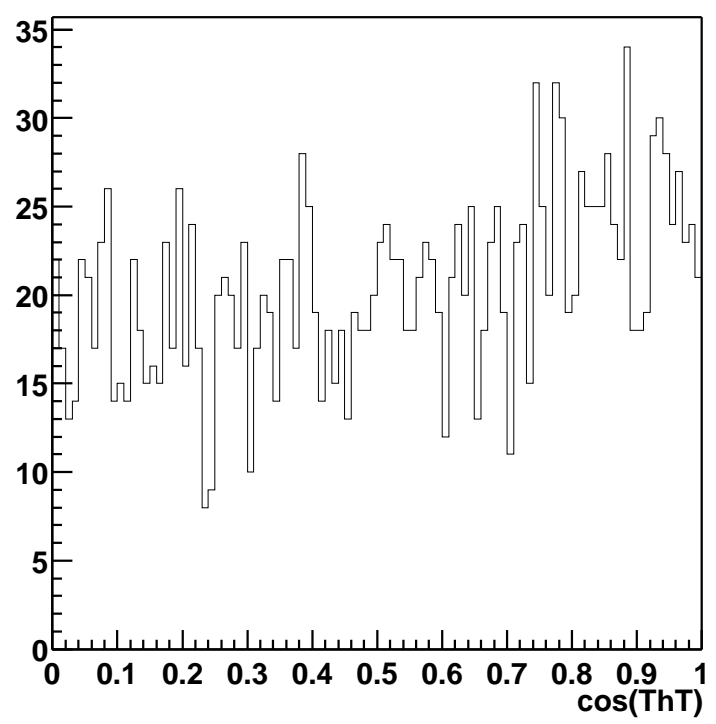

Continuum

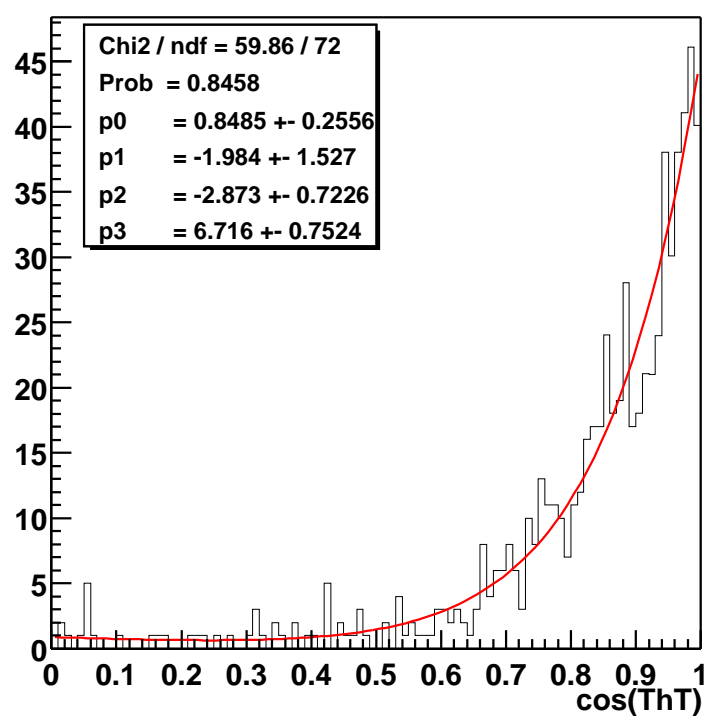

Figure 93: Distributions of $\left|\cos \theta_{T}\right|$ for $B \rightarrow X_{s} \mu^{+} \mu^{-}$candidates in truthmatched signal (top left), cross-feed (top right), $B \bar{B}$ (bottom left) and continuum (bottom right) events. The curves show the result of the fits to these distributions using the functions listed in Table 5. 

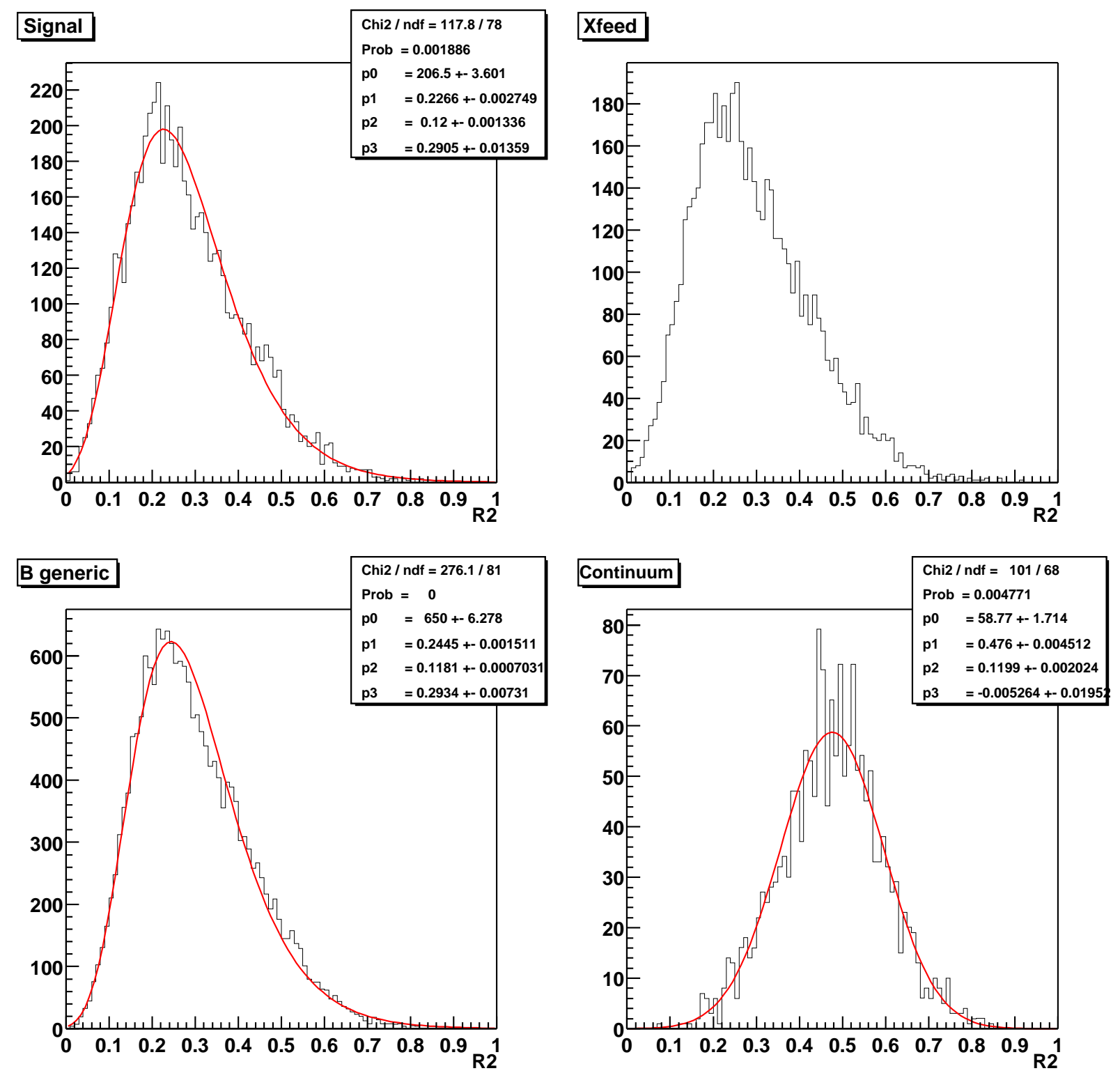

Figure 94: Distributions of $R_{2}$ for $B \rightarrow X_{s} \ell^{+} \ell^{-}$candidates in truth-matched signal (top left), cross-feed (top right), $B \bar{B}$ (bottom left) and continuum (bottom right) events. The curves show the result of the fits to these distributions using the functions listed in Table 5 . 
032796

\section{A P P E N D I X C}

\section{Gamma conversion veto}

The $e^{+} e^{-}$pairs originating from photon conversions in the detector material must be suppressed in the analysis. The $\gamma$ conversion code makes use of the decay topology of $\gamma$ conversions by finding $e^{+} e^{-}$pairs that have tangential tracks and small $m_{e^{+} e^{-}}$.

The module GammaToEE_Default uses $m_{e}$-assigned ChargedTracks to generate a list of $\gamma$ conversion candidates, gammaConversionDefault, with cuts on the distance between the two POCA $P_{1}$ and $P_{2}$ of the two tracks in the $(x, y)$ plane, $\left|\delta_{x y}\right|<0.5 \mathrm{~cm}$, the distance along the $z$ axis between $P_{1}$ and $P_{2},\left|\delta_{z}\right|<1.0 \mathrm{~cm}$, and $m_{e^{+} e^{-}}<30 \mathrm{MeV}$. Taking into account the location of the beam pipe, we also require that the radial distance in the $(x, y)$ plane between the decay vertex of the $\gamma$ conversion and the primary IP be $R_{x y}>2.0 \mathrm{~cm}$.

The $\gamma$ conversion veto is applied after the best $B$ candidate selection. In case at least one lepton in an $e^{+} e^{-}$pair of the $B$ candidate is part of a $\gamma$ conversion candidate's $e^{+} e^{-}$pair, the event is discarded. This veto removes about $x . x \%$ of reconstructed, truth matched signal $B \rightarrow X_{s} e^{+} e^{-}$candidates while decreasing the contamination in those decays by $\gamma$ conversion candidates to a negligible level. 


\section{BIBLIOGRAPHY}

[1] A. Ali, E. Lunghi, C. Greub, and G. Hiller. Improved model-independent analysis of semileptonic and radiative rare b decays. Phys. Rev., D66:034002, 2002 .

[2] K. Hagiwara et al. Review of particle physics. Phys. Rev., D66:010001, 2002.

[3] Ahmed Ali, Patricia Ball, L. T. Handoko, and G. Hiller. A comparative study of the decays $\mathrm{b} \rightarrow\left(\mathrm{k}, \mathrm{k}^{*}\right) \mathrm{l}+\mathrm{l}$ - in standard model and supersymmetric theories. Phys. Rev., D61:074024, 2000.

[4] S. Weinberg. A model of leptons. Phys. Rev. Lett., 19:1264-1266, 1967.

[5] A. Salam. Elementary Particle Theory. Almquist and Wiksell, Stockholm, 1968.

[6] Elliot Leader and Enrico Predazzi. An Introduction to Gauge Theories and Modern Particle Physics, volume 1. Cambridge University Press, 1996.

[7] N. Cabibbo. Unitary symmetry and leptonic decays. Phys. Rev. Lett., 10:531$532,1963$.

[8] M. Kobayashi and T. Maskawa. Cp violation in the renormalizable theory of weak interaction. Prog. Theor. Phys., 49:652-657, 1973.

[9] C. S. Wu, E. Ambler, R. W. Hayward, D. D. Hoppes, and R. P. Hudson. Experimental test of parity conservation in beta decay. Phys. Rev., 105:1413$1414,1957$.

[10] M. Goldhaber, L. Grodzins, and A. W. Sunyar. Helicity of neutrinos. Phys. Rev., 109:1015-1017, 1958.

[11] J. H. Christenson, J. W. Cronin, V. L. Fitch, and R. Turlay. Evidence for the 2 pi decay of the $\mathrm{k}(2) 0$ meson. Phys. Rev. Lett., 13:138-140, 1964.

[12] B. Aubert et al. Observation of cp violation in the b0 meson system. Phys. Rev. Lett., 87:091801, 2001. 
[13] K. Abe et al. Observation of large cp violation in the neutral b meson system. Phys. Rev. Lett., 87:091802, 2001.

[14] A. D. Sakharov. Violation of cp invariance, c asymmetry, and baryon asymmetry of the universe. Pisma Zh. Eksp. Teor. Fiz., 5:32-35, 1967.

[15] Andrzej J. Buras. Weak hamiltonian, cp violation and rare decays. 1998.

[16] Karen Lingel, Tomasz Skwarnicki, and James G. Smith. Penguin decays of b mesons. Ann. Rev. Nucl. Part. Sci., 48:253, 1998.

[17] Tobias Hurth. Present status of inclusive rare b decays. Rev. Mod. Phys., 75:1159-1199, 2003.

[18] Christoph Bobeth, Mikolaj Misiak, and Jorg Urban. Photonic penguins at two loops and $\mathrm{m}(\mathrm{t})$-dependence of $\mathrm{br}(\mathrm{b} \rightarrow \mathrm{x}(\mathrm{s}) \mathrm{l}+\mathrm{l}-)$. Nucl. Phys., B574:291-330, 2000 .

[19] Gudrun Hiller. $\mathrm{b} \rightarrow \mathrm{s}$ l+ l- decays in and beyond the standard model. AIP Conf. Proc., 549:614-618, 2002.

[20] B. Aubert et al. The BABAR detector. Nucl. Instrum. Meth., A479:1-116, 2002.

[21] U. Langenegger. "Cut-based electron identification", bad \#90 (2001).

[22] S. Chen et al. Branching fraction and photon energy spectrum for $\mathrm{b}-i \mathrm{~s}$ gamma. Phys. Rev. Lett., 87:251807, 2001.

[23] Geoffrey C. Fox and Stephen Wolfram. Observables for the analysis of event shapes in e+ e- annihilation and other processes. Phys. Rev. Lett., 41:1581, 1978.

[24] H. Albrecht et al. Reconstruction of b mesons. Phys. Lett., B185:218, 1987.

[25] A. Ali and G. Hiller. Eur. Phys. Jour. C 8. page 619, 1999.

[26] A. Telnov and A. Gritsan. " $K_{S}^{0} \rightarrow \pi^{+} \pi^{-}$Reconstruction Efficiency in BABAR: Release-10 Data vs. SP4 Monte Carlo", bad \#677 (2003).

[27] J. Berryhill, F. Porter, J. Richman, A. Ryd, and A. Samuel. "Observation of the rare decays $B \rightarrow K \ell^{+} \ell^{-}$and $B \rightarrow K^{*} \ell^{+} \ell^{-}$", bad \#559 (2003).

[28] S. Chen et al. Phys. Rev. Lett. 87, 251807. 2001.

[29] D. Cronin-Hennessy et al. Phys. Rev. Lett. 87, 251808. 2001.

[30] A. Ali and G. Hiller. Phys. Rev. D 60, 034017. 1999. 
[31] F. Di Lodovico, A. Eichenbaum, E. Grauges, S. Playfer, T. Pulliam, and J. Tinslay. "Study of $b \rightarrow$ s $\gamma$ using the sum of exclusive modes", bad \#220 (2002).

[32] A. Ali. hep-ph/0210183, cern-th/2002-284, october 2002.

[33] A. Ghinculov, T. Hurth, G. Isidori, and Y.P. Yao. hep-ph/0312128, slac-pub10265 (2003). 